\title{
Peptide View
}

MS/MS Fragmentation of ADVSVDQSKLPGVK

Found in gi|71043970|ref|NP_001020786.1|, Ymer protein isoform 2 [Mus musculus]

Match to Query 462: 1483.799848 from $(742.907200,2+)$

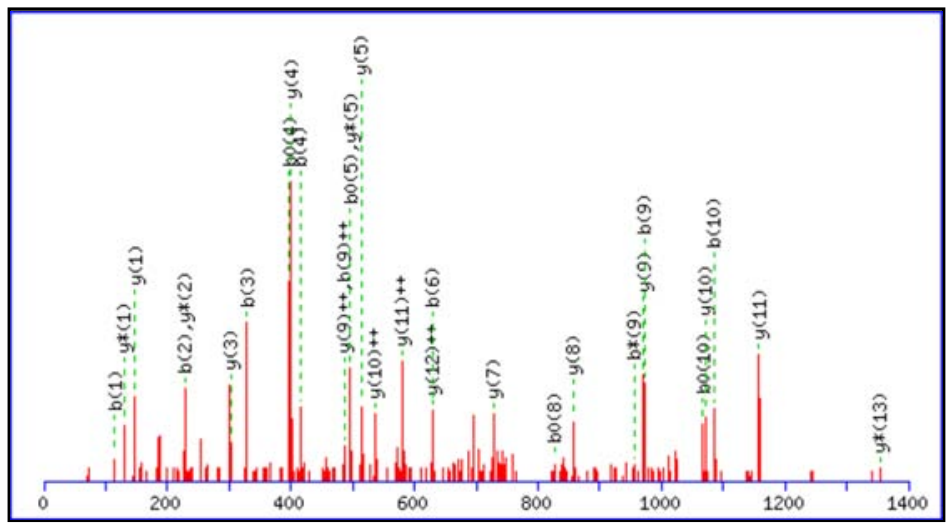

Monoisotopic mass of neutral peptide $\operatorname{Mr}(\mathrm{calc}): 1483.7882$

Variable modifications:

$\mathrm{N}$-term : N-Acetyl (Protein)

Ions Score: 55 Expect: 0.0049

Matches (Bold Red): 30/130 fragment ions using 50 most intense peaks

\begin{tabular}{|c|c|c|c|c|c|c|c|c|c|c|c|c|c|c|}
\hline \# & b & $b^{++}$ & $b *$ & $\mathrm{~b} *^{++}$ & $b^{0}$ & $b^{0++}$ & Seq. & y & $\mathrm{y}^{++}$ & $y *$ & $y *^{++}$ & $y^{0}$ & $y^{0++}$ & $\#$ \\
\hline 1 & 114.0549 & 57.5311 & & & & & A & & & & & & & 14 \\
\hline 2 & 229.0819 & 115.0446 & & & 211.0713 & 106.0393 & $\mathrm{D}$ & 1371.7478 & 686.3775 & 51354.7212 & 677.8643 & 1353.7372 & 2677.3723 & 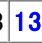 \\
\hline 3 & 328.1503 & 164.5788 & & & 310.1397 & 7155.5735 & V & 1256.7209 & 628.8641 & 11239.6943 & 620.3508 & 1238.7103 & 3619.8588 & 12 \\
\hline 4 & 415.1823 & 208.0948 & & & 7.1718 & 199.0895 & $\mathrm{~s}$ & 1157.6524 & 579.3299 & $\begin{array}{l}9 \\
\end{array}$ & 570.8166 & 1139.6419 & 9570.3246 & \\
\hline 5 & 514.2507 & 257.6290 & & & 496.2402 & 248.6237 & V & 1070.6204 & 535.8138 & \begin{tabular}{|l|l|}
8 & 1053.5939 \\
\end{tabular} & 527.3006 & 1052.6098 & \begin{tabular}{|l|l|}
826.8086 \\
\end{tabular} & 10 \\
\hline 6 & 629.2777 & 315.1425 & & & 611.2671 & 306.1372 & $\mathrm{D}$ & 971.5520 & 486.2796 & 954.5255 & 477.7664 & 953.5414 & $4 \quad 477.2744$ & \\
\hline 7 & 757.3363 & 379.1718 & 740.3097 & 370.6585 & 739.3257 & 370.1665 & $Q$ & 856.5251 & 428.7662 & \begin{tabular}{|l|l|}
2 & 839.4985 \\
\end{tabular} & 420.2529 & 838.5145 & 5419.7609 & 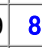 \\
\hline 8 & 844.3683 & 422.6878 & 827.3417 & 414.1745 & 826.3577 & 413.6825 & $\mathrm{~s}$ & 728.4665 & 364.7369 & \begin{tabular}{|l|l}
9 & 711.4399 \\
\end{tabular} & 356.2236 & 710.4559 & 9355.7316 & 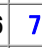 \\
\hline 9 & 972.4632 & 486.7353 & 955.4367 & 478.2220 & 954.4527 & 477.7300 & $\mathrm{~K}$ & 641.4345 & 321.2209 & 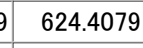 & 312.7076 & & & \\
\hline 10 & 1085.5473 & 543.2773 & 1068.5208 & 534.7640 & 1067.5367 & 534.2720 & $\mathrm{~L}$ & 513.3395 & 257.1734 & $4 \quad 496.3129$ & 248.6601 & & & \\
\hline 11 & 1182.6001 & 591.8037 & 1165.5735 & 583.2904 & 1164.5895 & 582.7984 & $\mathrm{P}$ & 400.2554 & 200.6314 & \begin{tabular}{l|l|}
4 & 383.2289 \\
\end{tabular} & 192.1181 & & & \\
\hline 12 & 1239.6215 & 620.3144 & 1222.5950 & 611.8011 & 1221.6110 & 611.3091 & $G$ & 303.2027 & 152.1050 & \begin{tabular}{|l|l|}
0 & 286.1761 \\
\end{tabular} & 143.5917 & & & \\
\hline 13 & 1338.6899 & 669.8486 & 1321.6634 & 661.3353 & 1320.6794 & 660.8433 & V & 246.1812 & 123.5942 & 2229.1547 & 115.0810 & & & \\
\hline 14 & & & & & & & $\mathrm{~K}$ & 147.1128 & 74.0600 & 130.0863 & 65.5468 & & & \\
\hline
\end{tabular}
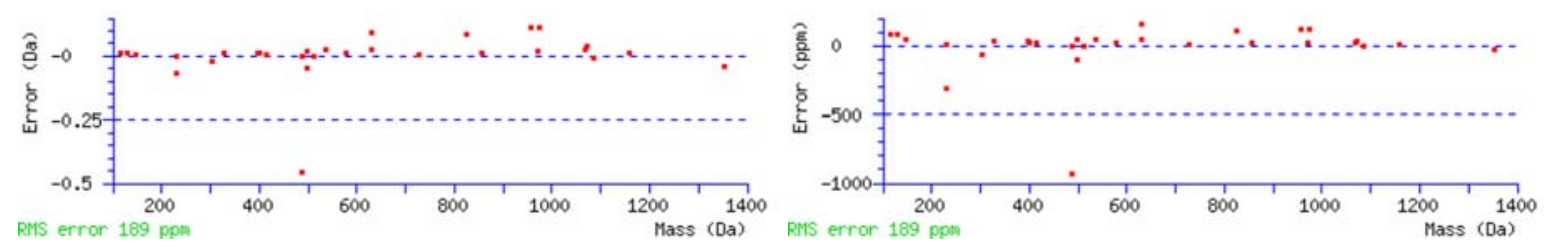


\section{Peptide View}

MS/MS Fragmentation of ALMVEWTDEFKNDPQLSLISAMIK

Found in gi|6755668|ref|NP_035614.1|, signal transducing adaptor molecule (SH3 domain and ITAM motif) 1 [Mus musculus]

Match to Query 891: 2778.630072 from $(927.217300,3+)$

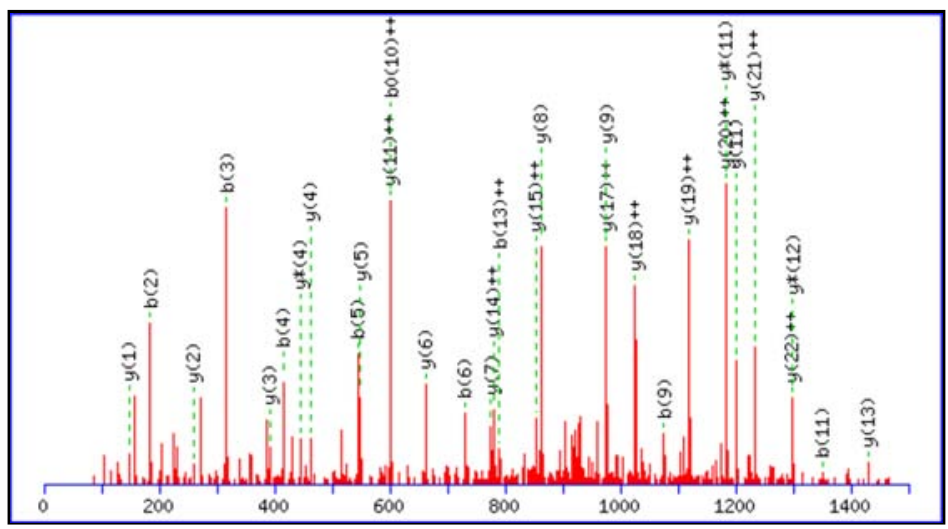

Monoisotopic mass of neutral peptide $\operatorname{Mr}(\mathrm{calc}): 2778.3972$

Ions Score: 53 Expect: 0.037

Matches (Bold Red): 32/240 fragment ions using 88 most intense peaks

\begin{tabular}{|c|c|c|c|c|c|c|c|c|c|c|c|c|c|c|}
\hline \# & b & $b^{++}$ & b* & $b *^{++}$ & $b^{0}$ & $b^{0++}$ & Seq. & $\mathbf{y}$ & $y^{++}$ & y* & $y *^{++}$ & $y^{0}$ & $y^{0++}$ & $\#$ \\
\hline 1 & 72.0444 & 36.5258 & & & & & A & & & & & & & 24 \\
\hline 2 & 185.1284 & 93.0679 & & & & & L & 2708.3673 & 1354.6873 & 2691.3408 & 1346.1740 & 2690.3568 & 1345.6820 & 23 \\
\hline 3 & 316.1689 & 158.5881 & & & & & M & 2595.2833 & 1298.1453 & 2578.2567 & 1289.6320 & 2577.2727 & 1289.1400 & 22 \\
\hline 4 & 415.2373 & 208.1223 & & & & & V & 2464.2428 & 1232.6250 & 2447.2162 & 1224.1118 & 2446.2322 & 1223.6198 & 21 \\
\hline 5 & 544.2799 & 272.6436 & & & 526.2694 & 263.6383 & $\mathrm{E}$ & 2365.1744 & 1183.0908 & 2348.1478 & 1174.5776 & 2347.1638 & 1174.0855 & 20 \\
\hline 6 & 730.3592 & 365.6833 & & & 712.3487 & 356.6780 & W & 2236.1318 & 1118.5695 & 2219.1052 & 1110.0563 & 2218.1212 & 1109.5643 & 19 \\
\hline 7 & 831.4069 & 416.2071 & & & 813.3964 & 407.2018 & $T$ & 2050.0525 & 1025.5299 & 2033.0259 & 1017.0166 & 2032.0419 & 1016.5246 & 18 \\
\hline 8 & 946.4339 & 473.7206 & & & 928.4233 & 464.7153 & D & 1949.0048 & 975.0060 & 1931.9783 & 966.4928 & 1930.9942 & 966.0008 & 17 \\
\hline 9 & 1075.4764 & 538.2419 & & & 1057.4659 & 529.2366 & $\mathrm{E}$ & 1833.9779 & 917.4926 & 1816.9513 & 908.9793 & 1815.9673 & 908.4873 & 16 \\
\hline 10 & 1222.5449 & 611.7761 & & & 1204.5343 & \begin{tabular}{|l|}
602.7708 \\
\end{tabular} & $\mathrm{~F}$ & 1704.9353 & 852.9713 & 1687.9087 & 844.4580 & 1686.9247 & 843.9660 & 15 \\
\hline 11 & 1350.6398 & 675.8235 & 1333.6133 & 667.3103 & 1332.6293 & 666.8183 & K & 1557.8669 & 779.4371 & 1540.8403 & 770.9238 & 1539.8563 & 770.4318 & 14 \\
\hline 12 & 1464.6827 & 732.8450 & 1447.6562 & 724.3317 & 1446.6722 & 723.8397 & $\mathrm{~N}$ & 1429.7719 & 715.3896 & 1412.7454 & 706.8763 & 1411.7613 & 706.3843 & 13 \\
\hline 13 & 1579.7097 & 790.3585 & 1562.6831 & 781.8452 & 1561.6991 & 781.3532 & D & 1315.7290 & 658.3681 & 1298.7024 & 649.8548 & 1297.7184 & 649.3628 & 12 \\
\hline 14 & 1676.7624 & 838.8849 & 1659.7359 & 830.3716 & 1658.7519 & 829.8796 & $P$ & 1200.7020 & 600.8547 & 1183.6755 & 592.3414 & 1182.6915 & 591.8494 & 11 \\
\hline 15 & 1804.8210 & 902.9142 & 1787.7945 & 894.4009 & 1786.8105 & 893.9089 & $\mathbf{Q}$ & 1103.6493 & 552.3283 & 1086.6227 & 543.8150 & 1085.6387 & 543.3230 & 10 \\
\hline 16 & 1917.9051 & 959.4562 & 1900.8785 & 950.9429 & 1899.8945 & 950.4509 & L & 975.5907 & 488.2990 & 958.5641 & 479.7857 & 957.5801 & 479.2937 & 9 \\
\hline 17 & 2004.9371 & 1002.9722 & 1987.9106 & 994.4589 & 1986.9266 & 993.9669 & $\mathrm{~s}$ & 862.5066 & 431.7570 & 845.4801 & 423.2437 & 844.4961 & 422.7517 & 8 \\
\hline 18 & 2118.0212 & 1059.5142 & 2100.9946 & 1051.0010 & 2100.0106 & 1050.5089 & L & 775.4746 & 388.2409 & 758.4481 & 379.7277 & 757.4640 & 379.2357 & 7 \\
\hline 19 & 2231.1052 & 1116.0563 & 2214.0787 & 1107.5430 & 2213.0947 & 1107.0510 & I & 662.3905 & 331.6989 & 645.3640 & 323.1856 & 644.3800 & 322.6936 & 6 \\
\hline 20 & 2318.1373 & 1159.5723 & 2301.1107 & 1151.0590 & 2300.1267 & 1150.5670 & $\mathrm{~s}$ & 549.3065 & 275.1569 & 532.2799 & 266.6436 & 531.2959 & 266.1516 & 5 \\
\hline 21 & 2389.1744 & 1195.0908 & 2372.1478 & 1186.5776 & 2371.1638 & 1186.0855 & A & 462.2745 & 231.6409 & 445.2479 & 223.1276 & & & 4 \\
\hline 22 & 2520.2149 & 1260.6111 & 2503.1883 & 1252.0978 & 2502.2043 & 1251.6058 & $M$ & 391.2373 & 196.1223 & 374.2108 & 187.6090 & & & 3 \\
\hline 23 & 2633.2989 & 1317.1531 & 2616.2724 & 1308.6398 & 2615.2884 & 1308.1478 & I & 260.1969 & 130.6021 & 243.1703 & 122.0888 & & & 2 \\
\hline 24 & & & & & & & $\mathrm{~K}$ & 147.1128 & 74.0600 & 130.0863 & 65.5468 & & & 1 \\
\hline
\end{tabular}
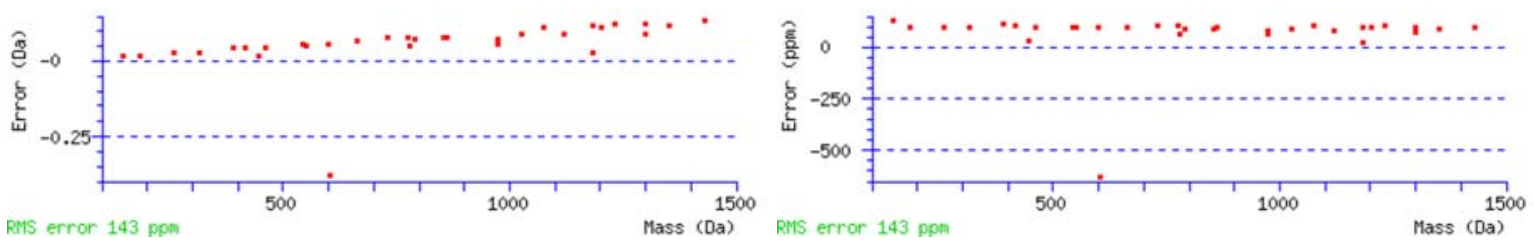


\section{Peptide View}

MS/MS Fragmentation of AMSTTSVTSSQPGK

Found in gi|7304993|ref|NP_038838.1|, drebrin-like [Mus musculus]

Match to Query 378: 1380.720648 from(691.367600,2+)

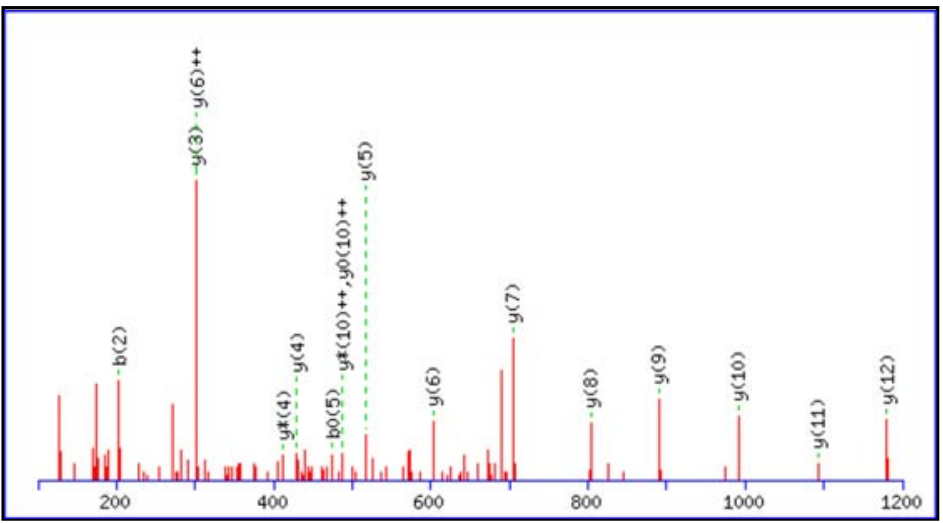

Monoisotopic mass of neutral peptide $\operatorname{Mr}(\mathrm{calc}): 1380.6555$

Ions Score: 90 Expect: $1.6 \mathrm{e}-006$

Matches (Bold Red): 16/124 fragment ions using 18 most intense peaks

\begin{tabular}{|c|c|c|c|c|c|c|c|c|c|c|c|c|c|c|}
\hline$\#$ & b & $\mathrm{b}^{++}$ & b* & $\mathrm{b} *^{++}$ & $b^{0}$ & $\mathrm{~b}^{0++}$ & Seq. & $y$ & $y^{++}$ & y* & $y *^{++}$ & $y^{0}$ & $y^{0++}$ & \# \\
\hline 1 & 72.0444 & 36.5258 & & & & & A & & & & & & & 14 \\
\hline 2 & 203.0849 & 102.0461 & & & & & $M$ & 1310.6257 & 655.8165 & 1293.5991 & 647.3032 & 1292.6151 & 646.8112 & 13 \\
\hline 3 & 290.1169 & 145.5621 & & & 2.1063 & 136.5568 & $\mathrm{~s}$ & 1179.5852 & 590.2962 & 1162.5586 & 581.7829 & 1161.5746 & 581.2909 & 12 \\
\hline 4 & 391.1646 & 196.0859 & & & 3.1540 & 187.0806 & $T$ & 1092.5531 & 546.7802 & 1075.5266 & 538.2669 & 1074.5426 & 537.7749 & 11 \\
\hline 5 & 492.2123 & 246.6098 & & & 474.2017 & 237.6045 & $T$ & 991.5055 & 496.2564 & 974.4789 & 487.7431 & 973.4949 & 487.2511 & 10 \\
\hline 6 & 579.2443 & 290.1258 & & & 561.2337 & 281.1205 & S & 890.4578 & 445.7325 & 873.4312 & 437.2193 & 872.4472 & 436.7272 & 9 \\
\hline 7 & 678.3127 & 339.6600 & & & 660.3021 & 330.6547 & V & 803.4258 & 402.2165 & 786.3992 & 393.7032 & 785.4152 & 393.2112 & 8 \\
\hline 8 & 779.3604 & 390.1838 & & & 761.3498 & 381.1785 & $T$ & 704.3573 & 352.6823 & 687.3308 & 344.1690 & 686.3468 & 343.6770 & 7 \\
\hline 9 & 866.3924 & 433.6998 & & & 848.3818 & 424.6946 & s & 603.3097 & 302.1585 & 586.2831 & 293.6452 & 585.2991 & 293.1532 & 6 \\
\hline 10 & 953.4244 & 477.2159 & & & 935.4139 & 468.2106 & s & 516.2776 & 258.6425 & 499.2511 & 250.1292 & 498.2671 & 249.6372 & 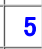 \\
\hline 11 & 1081.4830 & 541.2451 & 1064.4565 & 532.7319 & 1063.4725 & 532.2399 & $Q$ & 429.2456 & 215.1264 & 412.2191 & 206.6132 & & & 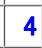 \\
\hline \begin{tabular}{|l|}
12 \\
\end{tabular} & 1178.5358 & 589.7715 & 1161.5092 & 581.2583 & 1160.5252 & 580.7662 & $P$ & 301.1870 & 151.0971 & 284.1605 & 142.5839 & & & 3 \\
\hline 13 & 1235.5572 & 618.2823 & 1218.5307 & 609.7690 & 1217.5467 & 609.2770 & G & 204.1343 & 102.5708 & 187.1077 & 94.0575 & & & - \\
\hline 14 & & & & & & & $\mathrm{~K}$ & 147.1128 & 74.0600 & 130.0863 & 65.5468 & & & 1 \\
\hline
\end{tabular}
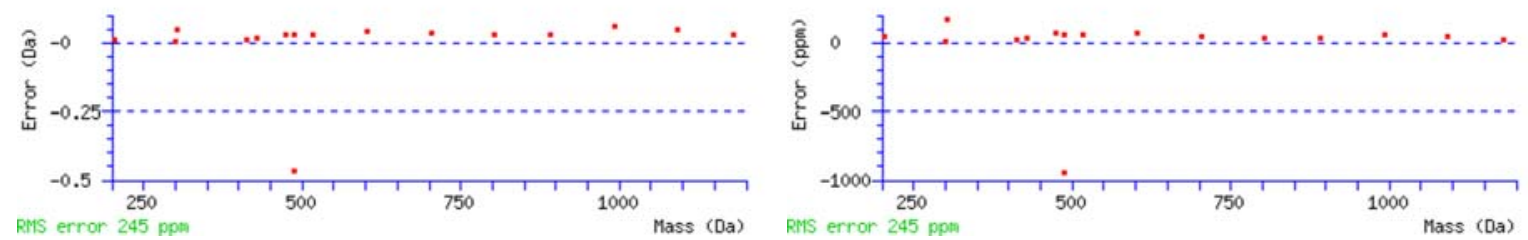


\section{Peptide View}

MS/MS Fragmentation of ARPLEEALDVIVSTFHK

Found in gi|33859624|ref|NP_035441.1|, S100 calcium binding protein A4 [Mus musculus]

Match to Query 485: 1976.159472 from(659.727100,3+)

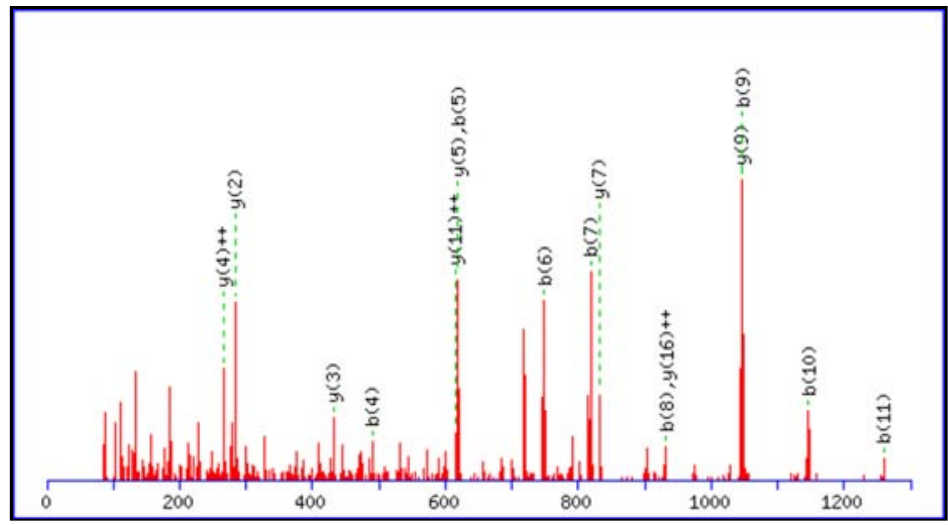

Monoisotopic mass of neutral peptide $\operatorname{Mr}$ (calc): 1976.0859

Variable modifications:

N-term : N-Acetyl (Protein)

R2 : Arginine-13C615N4 (R-full)

Ions Score: 64 Expect: 0.0016

Matches (Bold Red): 16/176 fragment ions using 24 most intense peaks

\begin{tabular}{|c|c|c|c|c|c|c|c|c|c|c|c|c|c|c|}
\hline \# & b & $b^{++}$ & b* & $\mathrm{b} *^{++}$ & $b^{0}$ & $b^{0++}$ & Seq. & $\mathbf{y}$ & $y^{++}$ & y* & $y *^{++}$ & $y^{0}$ & $y^{0++}$ & $\#$ \\
\hline 1 & 114.0549 & 57.5311 & & & & & A & & & & & & & 17 \\
\hline 2 & 280.1896 & 140.5984 & 263.1631 & 132.0852 & & & $\mathrm{R}$ & 1864.0455 & 932.5264 & 1847.0189 & 924.0131 & 1846.0349 & 923.5211 & 16 \\
\hline 3 & 377.2424 & 189.1248 & 360.2158 & 180.6115 & & & $P$ & 1697.9108 & 849.4590 & 1680.8843 & 840.9458 & 1679.9003 & 840.4538 & 15 \\
\hline 4 & 490.3264 & 245.6669 & 473.2999 & 237.1536 & & & L & 1600.8581 & 800.9327 & 1583.8315 & 792.4194 & 1582.8475 & 791.9274 & 14 \\
\hline 5 & 619.3690 & 310.1881 & 602.3425 & 301.6749 & 601.3585 & 301.1829 & E & 1487.7740 & 744.3906 & 1470.7475 & 735.8774 & 1469.7634 & 735.3854 & 13 \\
\hline 6 & 748.4116 & 374.7094 & 731.3851 & 366.1962 & 730.4010 & 365.7042 & E & 1358.7314 & 679.8693 & 1341.7049 & 671.3561 & 1340.7208 & 670.8641 & 12 \\
\hline 7 & 819.4487 & 410.2280 & 802.4222 & 401.7147 & 801.4382 & 401.2227 & A & 1229.6888 & 615.3480 & 1212.6623 & 606.8348 & 1211.6783 & 606.3428 & 11 \\
\hline 8 & 932.5328 & 466.7700 & 915.5062 & 458.2568 & 914.5222 & 457.7647 & L & 1158.6517 & 579.8295 & 1141.6252 & 571.3162 & 1140.6411 & 570.8242 & 10 \\
\hline 9 & 1047.5597 & 524.2835 & 1030.5332 & 515.7702 & 1029.5492 & 515.2782 & D & 1045.5677 & 523.2875 & 1028.5411 & 514.7742 & 1027.5571 & 514.2822 & 9 \\
\hline 10 & 1146.6281 & 573.8177 & 1129.6016 & 565.3044 & 1128.6176 & 564.8124 & V & 930.5407 & 465.7740 & 913.5142 & 457.2607 & 912.5301 & 456.7687 & 8 \\
\hline 11 & 1259.7122 & 630.3597 & 1242.6856 & 621.8465 & 1241.7016 & 621.3545 & I & 831.4723 & 416.2398 & 814.4458 & 407.7265 & 813.4617 & 407.2345 & 7 \\
\hline 12 & 1358.7806 & 679.8939 & 1341.7541 & 671.3807 & 1340.7700 & 670.8887 & V & 718.3882 & 359.6978 & 701.3617 & 351.1845 & 700.3777 & 350.6925 & 6 \\
\hline 13 & 1445.8126 & 723.4100 & 1428.7861 & 714.8967 & 1427.8021 & 714.4047 & $S$ & 619.3198 & 310.1636 & 602.2933 & 301.6503 & 601.3093 & 301.1583 & 5 \\
\hline 14 & 1546.8603 & 773.9338 & 1529.8338 & 765.4205 & 1528.8497 & 764.9285 & $\mathrm{~T}$ & 532.2878 & 266.6475 & 515.2613 & 258.1343 & 514.2772 & 257.6423 & 4 \\
\hline 15 & 1693.9287 & 847.4680 & 1676.9022 & 838.9547 & 1675.9182 & 838.4627 & $F$ & 431.2401 & 216.1237 & 414.2136 & 207.6104 & & & 3 \\
\hline 16 & 1830.9876 & 915.9975 & 1813.9611 & 907.4842 & 1812.9771 & 906.9922 & $\mathrm{H}$ & 284.1717 & 142.5895 & 267.1452 & 134.0762 & & & 2 \\
\hline 17 & & & & & & & $\mathrm{~K}$ & 147.1128 & 74.0600 & 130.0863 & 65.5468 & & & 1 \\
\hline
\end{tabular}
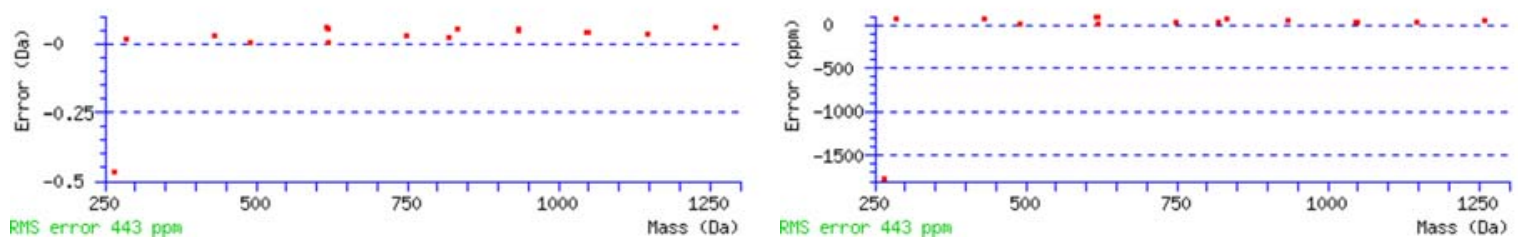


\section{Peptide View}

MS/MS Fragmentation of AVDLLNYTQWFPIVIFFNPDSR

Found in gi|7549795|ref|NP_035727.1|, tight junction protein 2 [Mus musculus]

Match to Query 786: 2660.383248 from(1331.198900,2+)

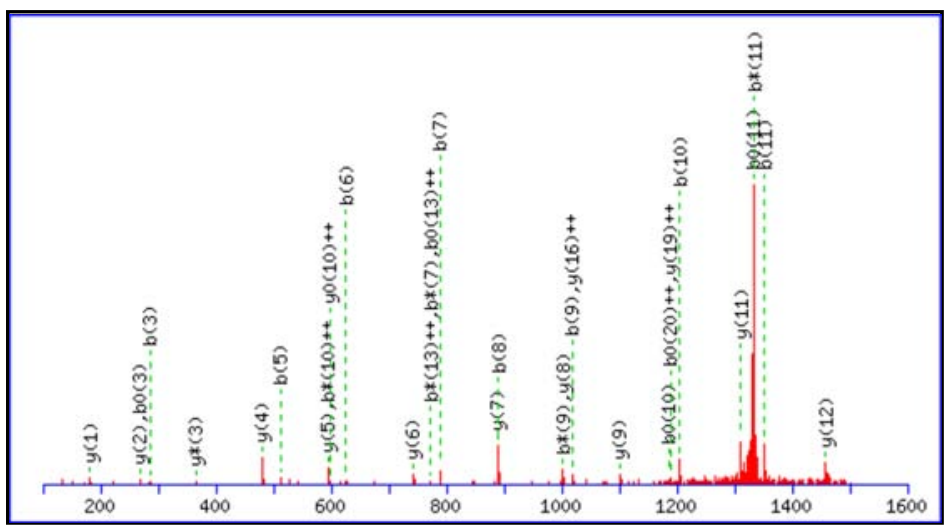

Monoisotopic mass of neutral peptide $\operatorname{Mr}$ (calc): 2660.3734

Variable modifications:

R22 : Arginine-13C6 (R-13C6)

Ions Score: 57 Expect: 0.016

Matches (Bold Red): 32/236 fragment ions using 66 most intense peaks

\begin{tabular}{|c|c|c|c|c|c|c|c|c|c|c|c|c|c|c|}
\hline$t$ & b & + & b* & $*^{++}$ & 0 & + & Seq. & y & ++ & $*$ & $y *^{++}$ & $y^{0}$ & $0++$ & $\#$ \\
\hline & 72 & & & & & & & & & & & & & 22 \\
\hline 2 & 171.1128 & 00 & & & & & & & & 0 & & 0 & & 21 \\
\hline 3 & 286.1397 & $73.5 / 35$ & & & & & & 2751 & 1246.1412 & 2474.2486 & 6279 & 2646 & 359 & 20 \\
\hline 4 & 238 & 155 & & & & & & & 77 & 23 & 145 & 376 & & 19 \\
\hline 5 & 512.3079 & 6.6576 & & & 4.2973 & 23 & L & 2263.1641 & 1132.0857 & 2246.1376 & 5724 & 2245.1536 & 804 & 18 \\
\hline 6 & 3508 & & 2 & & & & $\mathrm{~N}$ & & & & & & & 17 \\
\hline 7 & 789.4141 & 395.2107 & 2.3876 & 38 & 771.4 & 386 & Y & 2036.0372 & 1018 & 2019.0106 & 0089 & 266 & 169 & 16 \\
\hline 8 & .4618 & 570 & & & & & $\mathrm{~T}$ & & & & & & & 15 \\
\hline 9 & 1018.5204 & 509.7638 & 1001 & 1.2506 & 000.5098 & 500 & $Q$ & 177 & 67 & 17 & & 56 & 14 & 14 \\
\hline 0 & 1204.5997 & 2.8035 & 731 & 594.2902 & 1186.5891 & & W & 164 & & & & 570 & & 13 \\
\hline 1 & 1351.6681 & 676.3377 & 15 & & 133 & & 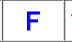 & 145 & & & 8845 & & & 12 \\
\hline 2 & 1448.7209 & 724.8641 & 31.6943 & 716.3508 & 1430.7103 & 715. & $P$ & 1310.7198 & 5.8636 & 129 & 03 & 7093 & 646 & 11 \\
\hline 3 & 1561.8049 & 1.4061 & 4.7784 & 772.8 & & & 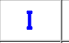 & & & & 8239 & 5565 & & 10 \\
\hline 4 & 1660.8733 & 830.9403 & 43.8468 & 822.4270 & 1642.8628 & 821.0 & V & 1100.5830 & 550.7952 & 1083.5565 & 542.2819 & 1082.5725 & 899 & 9 \\
\hline 5 & 1773.9574 & 887.4823 & 56.9308 & 878.9691 & 755.9468 & 878.4770 & 1 & 1001.5146 & 501.2609 & 984.4881 & 92.7477 & 983.5041 & 492.2557 & 0 \\
\hline 6 & 1921.0258 & 961.0165 & 03.9992 & 952.5033 & 1903.0152 & 952.0113 & $\mathrm{~F}$ & 88.4306 & 444.7189 & 71.4040 & 2056 & 870.4200 & 435.7136 & 7 \\
\hline 7 & 2068.0942 & \begin{tabular}{|l|}
1034.5507 \\
\end{tabular} & 51.0677 & \begin{tabular}{|l|}
1026.0375 \\
\end{tabular} & 2050.0836 & 1025.5455 & $r$ & 41.3621 & 371.1847 & 724.3356 & 362.6714 & 723.3516 & 362.1794 & 6 \\
\hline 18 & 2182.1371 & 1091.5722 & 165.1106 & 1083.0589 & 2164.1266 & 1082.5669 & $\mathrm{~N}$ & 594.2937 & 297.6505 & 577.2672 & 289.1372 & 576.2832 & 288.6452 & 5 \\
\hline 19 & \begin{tabular}{|l|}
2279.1899 \\
\end{tabular} & 1140.0986 & \begin{tabular}{|l|}
2262.1633 \\
\end{tabular} & 1131.5853 & 2261.1793 & 1131.0933 & $\mathrm{P}$ & 480.2508 & 240.6290 & 463.2243 & 232.1158 & 462.2402 & 231.6238 & 4 \\
\hline 20 & 2394.2168 & 1197.6121 & 2377.1903 & 1189.0988 & 2376.2063 & 1188.6068 & D & 383.1980 & 192.1027 & 366.1715 & 183.5894 & 365.1875 & 183.0974 & 3 \\
\hline 21 & 2481.2489 & 1241.1281 & 64.2223 & 1232.6148 & 2463.2383 & 1232. & 0 & 268.1711 & 134.5892 & & 126.0759 & 250.1605 & 125.5 & 2 \\
\hline 22 & & & & & & & $R$ & 181.1391 & 91.0732 & 164.1125 & 82.5599 & & & \\
\hline
\end{tabular}

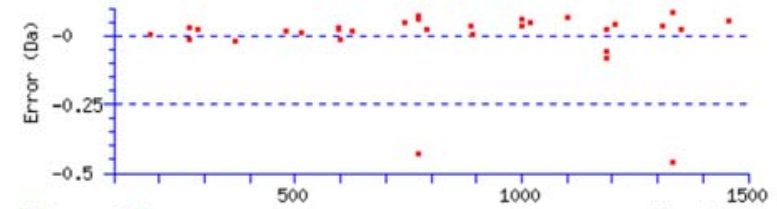

RMS error 125 ppom

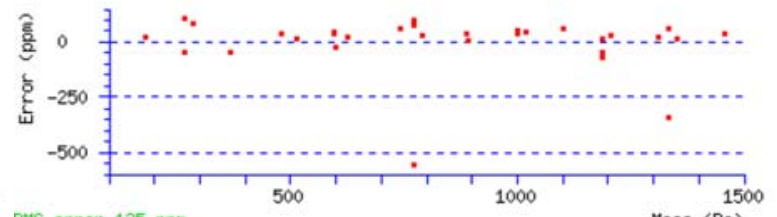

Mass (Da) 


\section{Peptide View}

MS/MS Fragmentation of EFATLIIDILSEAK

Found in gi|51921285|ref|NP_001004144.1|, G protein-coupled receptor kinase-interactor 1 [Mus musculus]

Match to Query 338: 1561.937048 from $(781.975800,2+)$

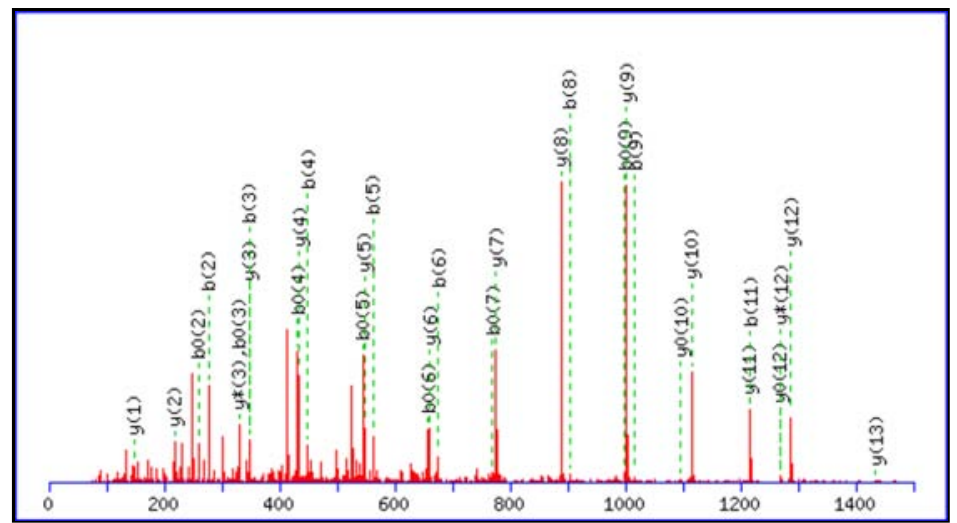

Monoisotopic mass of neutral peptide $\operatorname{Mr}(\mathrm{cal}): 1561.8603$

Ions Score: 68 Expect: 0.00033

Matches (Bold Red): 32/126 fragment ions using 90 most intense peaks

\begin{tabular}{|c|c|c|c|c|c|c|c|c|c|c|c|c|}
\hline \# & b & $\mathrm{b}^{++}$ & $b^{0}$ & $b^{0++}$ & Seq. & y & $\mathrm{y}^{++}$ & y* & $\mathrm{y}^{++}$ & $y^{0}$ & $y^{0++}$ & \# \\
\hline 1 & 130.0499 & 65.5286 & 2.0393 & 56.5233 & $\mathrm{E}$ & & & & & & & 14 \\
\hline 2 & 277.1183 & 139.0628 & 259.1077 & 130.0575 & $\mathrm{~F}$ & 50 & 61 & 1 & & 1144 & 11 & 13 \\
\hline 3 & 54 & 4.5813 & 0.1448 & 165.5760 & A & 66 & 3.8819 & 00 & 3686 & 460 & & 12 \\
\hline 4 & 449.2031 & 225.1052 & 431.1925 & 216.0999 & 1 & 1215.7195 & 608.3634 & 1198.6929 & 599.8501 & 1197.7089 & 599 & 11 \\
\hline 5 & 371 & 281.6472 & 544.2766 & 272.6419 & L & & 557.8395 & & 549.3262 & 6612 & & 10 \\
\hline 6 & 675.3712 & 338.1892 & 657.3606 & 329.1839 & I & 01.5877 & 501.2975 & 5612 & 492.7842 & 83.5771 & 492.2922 & 9 \\
\hline 7 & 788.4552 & 394.7313 & 770.4447 & 385.7260 & I & 037 & 444.7555 & 4771 & 436.2422 & 870.4931 & 435.7502 & 8 \\
\hline 8 & 903 & 452.2447 & 885.4716 & 443.2394 & D & & 134 & & 37 & 090 & & 7 \\
\hline 9 & 1016.5662 & 508.7868 & 998.5557 & 499.7815 & I & 27 & 330.7000 & 661 & 322.1867 & 2.3821 & 947 & 6 \\
\hline 10 & 1129.6503 & 565.3288 & 1111.6397 & 556.3235 & L & 886 & 274.1579 & 2820 & 265.6447 & 529.2980 & 265.1527 & 5 \\
\hline 11 & 1216 & 608.8448 & 1198.6718 & 599. & s & 45 & 217.6159 & 980 & 1026 & 2140 & 106 & 4 \\
\hline 12 & 1345.7249 & 673.3661 & 1327.7144 & 664.3608 & $E$ & 1925 & 174.0999 & 330.1660 & 165.5866 & 329.1819 & 165.0946 & 3 \\
\hline 13 & 1416.7620 & 708.8847 & 1398.7515 & 699.8794 & A & & 109.5786 & 1234 & 101.0653 & & & 2 \\
\hline 14 & & & & & $\mathrm{~K}$ & 28 & 74.0600 & 363 & 468 & & & 1 \\
\hline
\end{tabular}
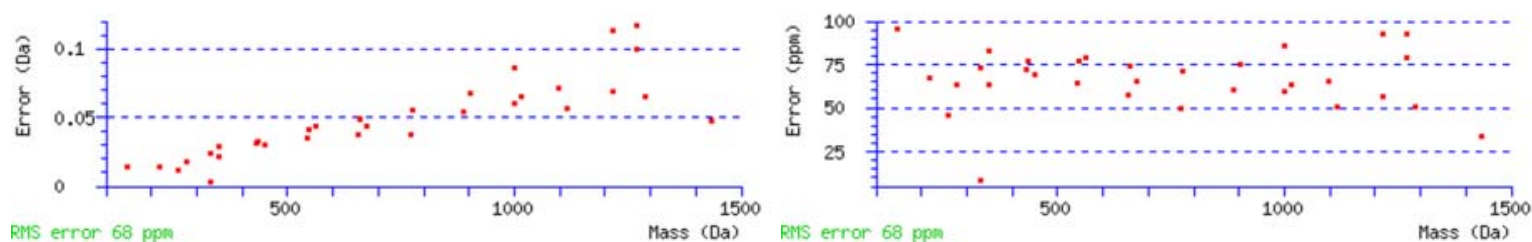

\footnotetext{
RMs error 68 ppon
} Mass (Da) RMS error 68 ppo 


\section{Peptide View}

MS/MS Fragmentation of ELFALDLVTGVLTIK

Found in gi|18087747|ref|NP_291059.1|, protocadherin gamma subfamily C, 3 [Mus musculus]

Match to Query 540: 1630.984648 from( $(816.499600,2+)$

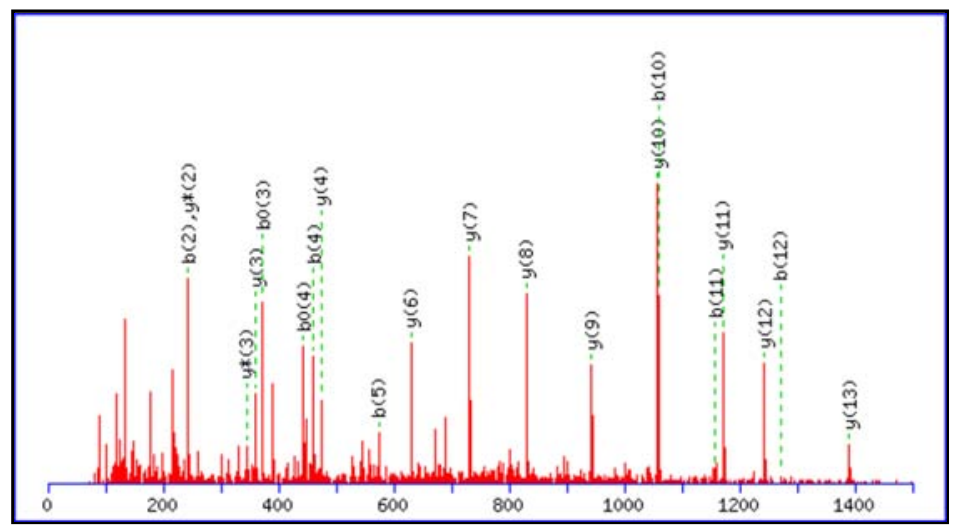

Monoisotopic mass of neutral peptide Mr (calc): 1630.9545

Ions Score: 70 Expect: 0.00025

Matches (Bold Red): 20/136 fragment ions using 43 most intense peaks

\begin{tabular}{|c|c|c|c|c|c|c|c|c|c|c|c|c|}
\hline \# & b & $b^{++}$ & $b^{0}$ & $b^{0++}$ & Seq. & y & $\mathrm{y}^{++}$ & y* & $y *^{++}$ & $y^{0}$ & $y^{0++}$ & \# \\
\hline 1 & 130.0499 & 65.5286 & 112.0393 & 56.5233 & $\mathrm{E}$ & & & & & & & 15 \\
\hline 2 & 243.1339 & 122.0706 & 225.1234 & 113.0653 & L & 1502.9192 & 751.9632 & 1485.8927 & 743.4500 & 1484.9086 & 174.00 & 14 \\
\hline 3 & 390.2023 & 195.6048 & 372.1918 & 186.5995 & $\mathrm{~F}$ & 138 & 695.4212 & 1372. & 686.9079 & 1371.8246 & 686.4159 & 13 \\
\hline 4 & 461.2394 & 231.1234 & 443.2289 & 222.1181 & A & 1242.7667 & 621.8870 & 1225.7402 & 613.3737 & 1224.7562 & 612.8817 & 712 \\
\hline 5 & 574.3235 & 287.6654 & 556.3129 & 278.6601 & L & 1171.7296 & 586.3684 & 1154.7031 & 577.8552 & 1153.7191 & 577.3632 & 11 \\
\hline 6 & 689.3504 & 345.1789 & 671.3399 & 336.1736 & D & 1058.6456 & 529.8264 & 1041.6190 & 521.3131 & 1040.6350 & 520.8211 & 10 \\
\hline 7 & 802.4345 & 401.7209 & 784.4239 & 392.7156 & $\mathrm{~L}$ & 943.6186 & 472.3129 & 926.5921 & 463.7997 & 925.6081 & 463.3077 & 9 \\
\hline 8 & 901.5029 & 451.2551 & 883.4924 & 442.2498 & V & 830.5346 & 415.7709 & 813.5080 & 407.2576 & 812.5240 & 406.7656 & 8 \\
\hline 9 & 1002.5506 & 501.7789 & 984.5400 & 492.7737 & $\mathrm{~T}$ & 731.4662 & 366.2367 & 714.4396 & 357.7234 & 713.4556 & 357.2314 & 7 \\
\hline 10 & 1059.5721 & 530.2897 & 1041.5615 & 521.2844 & G & 630.4185 & 315.7129 & 613.3919 & 307.1996 & 612.4079 & 306.7076 & 6 \\
\hline 11 & 1158.6405 & 579.8239 & 1140.6299 & 570.8186 & V & 573.3970 & 287.2021 & 556.3705 & 278.6889 & 555.3864 & 278.1969 & 5 \\
\hline 12 & 1271.7245 & 636.3659 & 1253.7140 & 627.3606 & L & 474.3286 & 237.6679 & 457.3021 & 229.1547 & 456.3180 & 228.6627 & 4 \\
\hline 13 & 1372.7722 & 686.8897 & 1354.7616 & 677.8845 & $T$ & 361.2445 & 181.1259 & 344.2180 & 172.6126 & 343.2340 & 172.1206 & 3 \\
\hline 14 & 1485.8563 & 743.4318 & 1467.8457 & 734.4265 & I & 260.1969 & 130.6021 & 243.1703 & 122.0888 & & & 2 \\
\hline 15 & & & & & $\mathrm{~K}$ & 147.1128 & 74.0600 & 130.0863 & 65.5468 & & & 1 \\
\hline
\end{tabular}
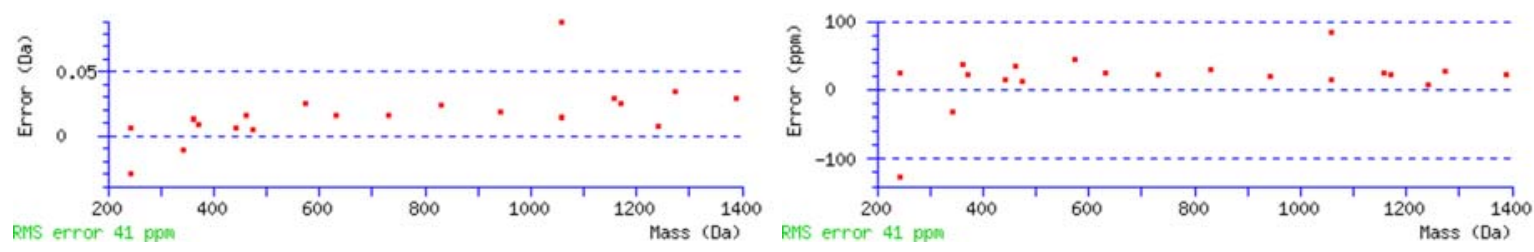


\section{Peptide View}

MS/MS Fragmentation of EQFALAMYFIQQK

Found in gi|6679669|ref|NP_031970.1|, epidermal growth factor receptor pathway substrate 15, related [Mus musculus]

Match to Query 924: 1615.898248 from $(808.956400,2+)$

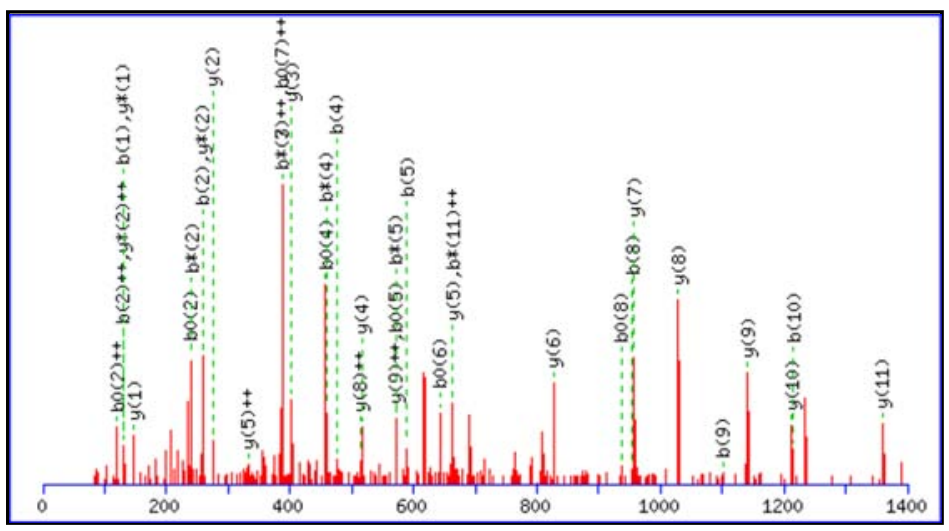

Monoisotopic mass of neutral peptide $\operatorname{Mr}(\mathrm{calc}): 1615.8068$

Ions Score: 51 Expect: 0.015

Matches (Bold Red): 39/118 fragment ions using 87 most intense peaks

\begin{tabular}{|c|c|c|c|c|c|c|c|c|c|c|c|c|}
\hline \# & b & $b^{++}$ & $b *$ & $\mathrm{~b} *^{++}$ & $b^{0}$ & $b^{0++}$ & Seq. & $y$ & $y^{++}$ & y* & $y *^{++}$ & \# \\
\hline 1 & 130.0499 & 65.5286 & & & 112.0393 & 56.5233 & $\mathrm{E}$ & & & & & 13 \\
\hline 2 & 258.1084 & 129.5579 & 241.0819 & 121.0446 & 240.0979 & 120.5526 & $Q$ & 1487.7715 & 744.3894 & 1470.7450 & 735.8761 & 12 \\
\hline 3 & 405.1769 & 203.0921 & 388.1503 & 194.5788 & 387.1663 & 194.0868 & $\mathrm{~F}$ & 1359.7129 & 680.3601 & 1342.6864 & 671.8468 & 11 \\
\hline 4 & 476.2140 & 238.6106 & 459.1874 & 230.0973 & 458.2034 & 229.6053 & A & 1212.6445 & 606.8259 & 1195.6180 & 598.3126 & 10 \\
\hline 5 & 589.2980 & 295.1527 & 572.2715 & 286.6394 & 571.2875 & 286.1474 & $\mathrm{~L}$ & 1141.6074 & 571.3073 & 1124.5809 & 562.7941 & 9 \\
\hline 6 & 660.3351 & 330.6712 & 643.3086 & 322.1579 & 642.3246 & 321.6659 & A & 1028.5234 & 514.7653 & 1011.4968 & 506.2520 & 8 \\
\hline 7 & 791.3756 & 396.1914 & 774.3491 & 387.6782 & 773.3651 & 387.1862 & $M$ & 957.4862 & 479.2468 & 940.4597 & 470.7335 & 7 \\
\hline 8 & 954.4389 & 477.7231 & 937.4124 & 469.2098 & 936.4284 & 468.7178 & $\mathrm{Y}$ & 826.4458 & 413.7265 & 809.4192 & 405.2132 & 6 \\
\hline 9 & 1101.5074 & 551.2573 & 1084.4808 & 542.7440 & 1083.4968 & 542.2520 & $\mathrm{~F}$ & 663.3824 & 332.1949 & 646.3559 & 323.6816 & 5 \\
\hline 10 & 1214.5914 & 607.7993 & 1197.5649 & 599.2861 & 1196.5809 & 598.7941 & I & 516.3140 & 258.6606 & 499.2875 & 250.1474 & 4 \\
\hline 11 & 1342.6500 & 671.8286 & 1325.6234 & 663.3154 & 1324.6394 & 662.8234 & $\mathbf{Q}$ & 403.2300 & 202.1186 & 386.2034 & 193.6053 & 3 \\
\hline 12 & 1470.7086 & 735.8579 & 1453.6820 & 727.3447 & 1452.6980 & 726.8526 & $Q$ & 275.1714 & 138.0893 & 258.1448 & 129.5761 & 2 \\
\hline 13 & & & & & & & $\mathrm{~K}$ & 147.1128 & 74.0600 & 130.0863 & 65.5468 & \begin{tabular}{l|l|}
8 & 1
\end{tabular} \\
\hline
\end{tabular}
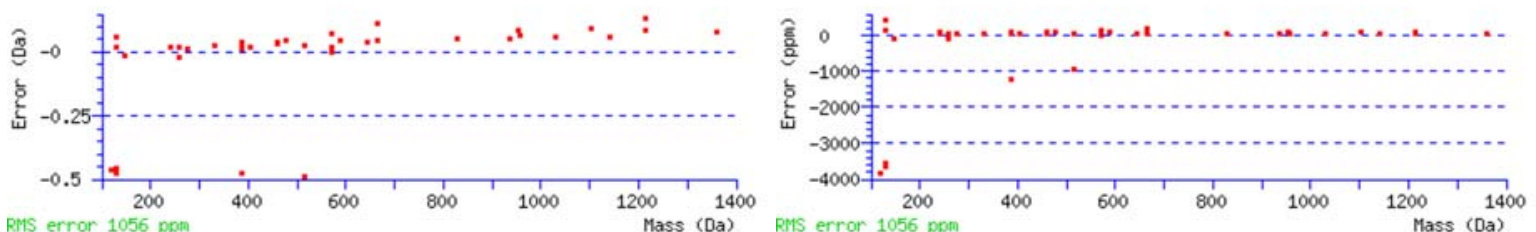


\section{Peptide View}

MS/MS Fragmentation of FFEVILIDPFHK

Found in gi|94385783|ref|XP_001004374.1|, PREDICTED: similar to ribosomal protein L15 [Mus musculus]

Match to Query 807: 1503.900648 from $(752.957600,2+)$

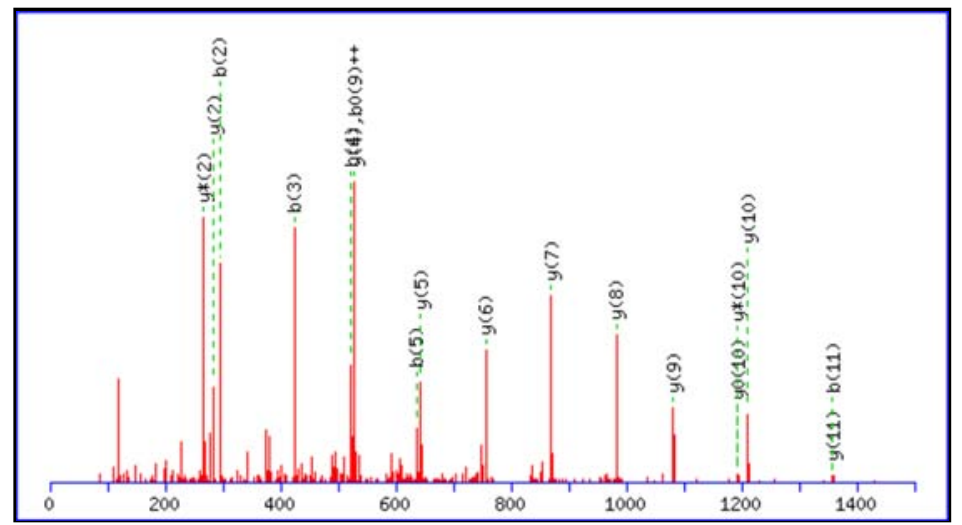

Monoisotopic mass of neutral peptide $\operatorname{Mr}(\mathrm{calc}): 1503.8125$

Ions Score: 60 Expect: 0.0018

Matches (Bold Red): 18/98 fragment ions using 40 most intense peaks

\begin{tabular}{|c|c|c|c|c|c|c|c|c|c|c|c|c|}
\hline \# & b & $\mathrm{b}^{++}$ & $b^{0}$ & $b^{0++}$ & Seq. & y & $y^{++}$ & $y *$ & $\mathrm{y}^{++}$ & $y^{0}$ & $y^{0++}$ & $\#$ \\
\hline 1 & 148.0757 & 74.5415 & & & $F$ & & & & & & & 12 \\
\hline 2 & 295.1441 & 148.0757 & & & $\mathrm{~F}$ & 1357.7514 & 679.3793 & 1340.7249 & 670.8661 & 1339.7408 & 670.3741 & 11 \\
\hline 3 & 424.1867 & 212.5970 & 406.1761 & 203.5917 & $E$ & 1210.6830 & 605.8451 & 1193.6565 & 597.3319 & 1192.6724 & 596.8399 & 10 \\
\hline 4 & 523.2551 & 262.1312 & 505.2445 & 253.1259 & V & 1081.6404 & 541.3238 & 1064.6139 & 532.8106 & 1063.6298 & 532.3186 & 9 \\
\hline 5 & 636.3392 & 318.6732 & 618.3286 & 309.6679 & I & 982.5720 & 491.7896 & 965.5455 & 483.2764 & 964.5614 & 482.7844 & 8 \\
\hline 6 & 749.4232 & 375.2152 & 731.4127 & 366.2100 & L & 869.4879 & 435.2476 & 852.4614 & 426.7343 & 851.4774 & 426.2423 & 7 \\
\hline 7 & 862.5073 & 431.7573 & 844.4967 & 422.7520 & I & 756.4039 & 378.7056 & 739.3773 & 370.1923 & 738.3933 & 369.7003 & 6 \\
\hline 8 & 977.5342 & 489.2707 & 959.5237 & 480.2655 & D & 643.3198 & 322.1635 & 626.2933 & 313.6503 & 625.3093 & 313.1583 & 5 \\
\hline 9 & 1074.5870 & 537.7971 & 1056.5764 & 528.7918 & $\mathrm{P}$ & 528.2929 & 264.6501 & 511.2663 & 256.1368 & & & 4 \\
\hline 10 & 1221.6554 & 611.3313 & 1203.6448 & 602.3260 & $\mathrm{~F}$ & 431.2401 & 216.1237 & 414.2136 & 207.6104 & & & 3 \\
\hline 11 & 1358.7143 & 679.8608 & 1340.7037 & 670.8555 & $\mathrm{H}$ & 284.1717 & 142.5895 & 267.1452 & 134.0762 & & & 2 \\
\hline 12 & & & & & $\mathrm{~K}$ & 147.1128 & 74.0600 & 130.0863 & 65.5468 & & & 1 \\
\hline
\end{tabular}
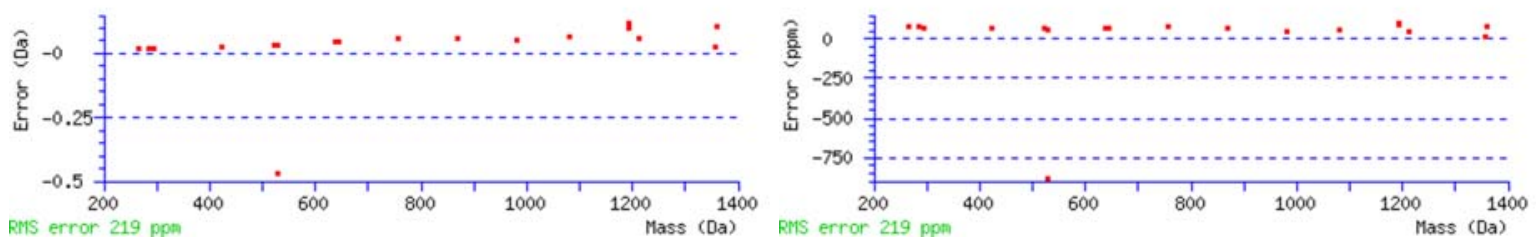


\section{Peptide View}

MS/MS Fragmentation of FGSAIPIPSLPDK

Found in gi|29568084|ref|NP_079940.2|, sorting nexin 9 [Mus musculus]

Match to Query 224: 1340.792648 from(671.403600,2+)

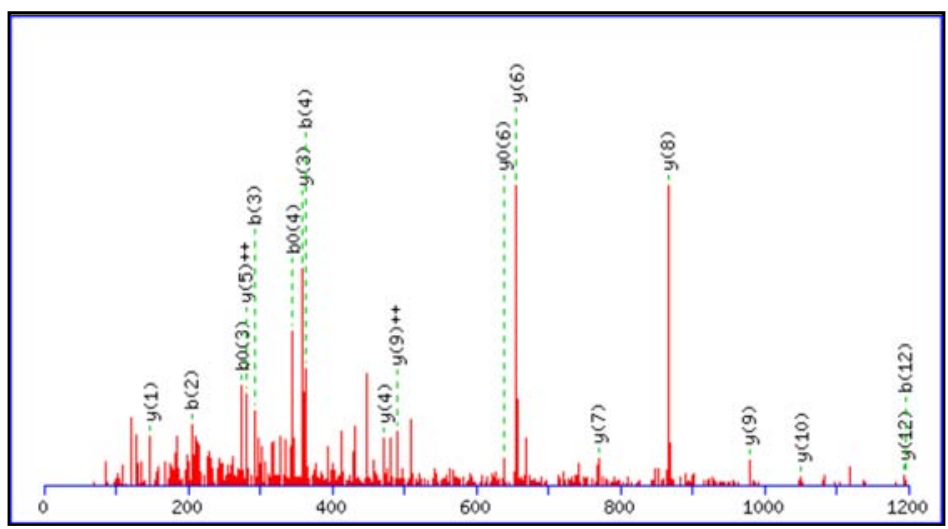

Monoisotopic mass of neutral peptide $\mathrm{Mr}$ (calc): 1340.7340

Ions Score: 46 Expect: 0.033

Matches (Bold Red) : 18/114 fragment ions using 42 most intense peaks

\begin{tabular}{|c|c|c|c|c|c|c|c|c|c|c|c|c|}
\hline \# & b & $b^{++}$ & $b^{0}$ & $b^{0++}$ & Seq. & $y$ & $y^{++}$ & y* & $y *^{++}$ & $y^{0}$ & $y^{0++}$ & \\
\hline 1 & 148.0757 & 74.5415 & & & $F$ & & & & & & & 13 \\
\hline 2 & 205.0971 & 103.0522 & & & $G$ & 4.6728 & & 1 & & 117 & & 12 \\
\hline 3 & 92.1292 & 146.5682 & 36 & 29 & $\mathrm{~s}$ & 3514 & 5 & 112 & 3160 & 408 & & \\
\hline 4 & 363.1663 & 182.0868 & 345.1557 & 173.0815 & $A$ & 1050.6193 & 525.8133 & 1033.5928 & 517.3000 & 1032 & 516 & 1 \\
\hline 5 & 476.2503 & 238.6288 & 58.2398 & 229.6235 & I & 822 & 490.2948 & 962.5557 & 481.7815 & 961.5717 & 481.2895 & \\
\hline 6 & 573.3031 & 287.1552 & 555.2925 & & $P$ & & 433.7527 & 16 & 394 & & 424 & \\
\hline 7 & 686.3872 & 343.6972 & 668.3766 & 334.6919 & I & 1454 & 385.2263 & 752.4189 & 376.7131 & 751.4348 & 376.2211 & \\
\hline 8 & 783.4399 & 392.2236 & 765.4294 & & $P$ & & 328.6843 & 639.3348 & & 638.3508 & 319.6790 & \\
\hline 9 & 870.4720 & 435.7396 & 852.4614 & 426.7343 & $S$ & 559.3086 & 280.1579 & 542.2820 & 271.6447 & 541.2980 & 271.1527 & 5 \\
\hline 0 & 983.5560 & 492.2816 & 965.5455 & 483.2764 & $L$ & 2766 & 236.6419 & 455.2500 & 228.1286 & 454.2660 & 227.6366 & 4 \\
\hline 11 & 1080.6088 & 540.8080 & 1062.5982 & 531.8027 & $P$ & 359.1925 & 180.0999 & 342.1660 & 171.5866 & 341.1819 & 171.0946 & 3 \\
\hline 2 & 1195.6357 & 598.3215 & 1177.6252 & 589.3162 & D & & 131.5735 & 245.1132 & 123.0602 & 244.1292 & 122.5682 & 2 \\
\hline 13 & & & & & $\mathrm{~K}$ & 147.1128 & 74.0600 & 130.0863 & 65.5468 & & & 1 \\
\hline
\end{tabular}
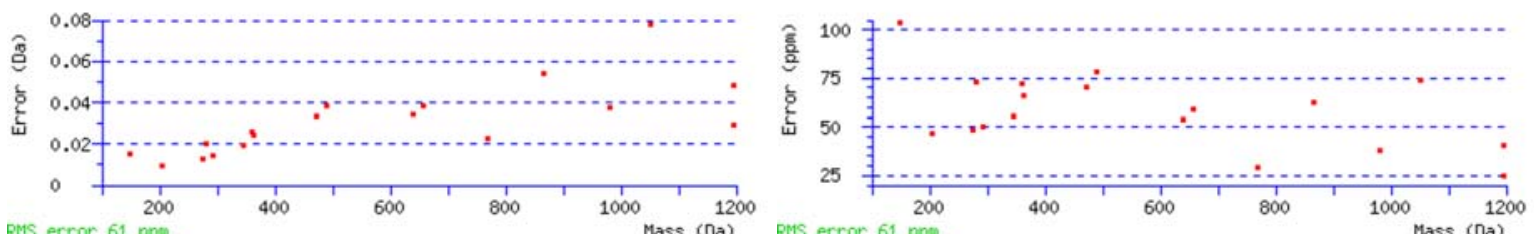

RMS error 61 ppe

Mass (Da) 


\section{Peptide View}

MS/MS Fragmentation of FGVEQDVDMVFASFIR

Found in gi|31981562|ref|NP_035229.2|, pyruvate kinase 3 [Mus musculus]

Match to Query 611: 1858.988248 from $\left(930.501400,2^{+}\right)$

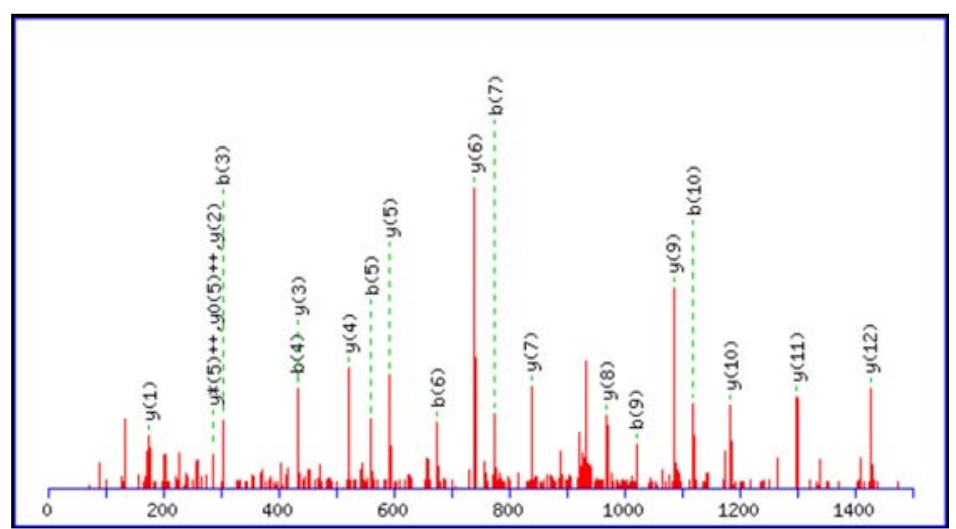

Monoisotopic mass of neutral peptide Mr(calc): 1858.8923

Ions Score: 88 Expect: 5.1e-006

Matches (Bold Red) : 21/160 fragment ions using 37 most intense peaks

\begin{tabular}{|c|c|c|c|c|c|c|c|c|c|c|c|c|c|c|}
\hline \# & b & $b^{++}$ & b* & $\mathrm{b} * *^{++}$ & $b^{0}$ & $b^{0++}$ & Seq. & y & $\mathrm{y}^{++}$ & y* & $\mathrm{y}^{++}$ & $y^{0}$ & $y^{0++}$ & \# \\
\hline 1 & 148.0757 & 74.5415 & & & & & $\mathrm{~F}$ & & & & & & & 16 \\
\hline 2 & 205.0971 & 103.0522 & & & & & G & 1712.8312 & 856.9192 & 1695.8046 & 848.4060 & 1694.8206 & 847.9140 & 15 \\
\hline 3 & 304.1656 & 152.5864 & & & & & V & 1655.8097 & 828.4085 & 1638.7832 & 819.8952 & 1637.7992 & 819.4032 & $\begin{array}{ll}2 & 14\end{array}$ \\
\hline 4 & 433.2081 & 217.1077 & & & 415.1976 & 208.1024 & $\mathrm{E}$ & 1556.7413 & 778.8743 & 1539.7148 & 770.3610 & 1538.7308 & 769.8690 & 13 \\
\hline 5 & 561.2667 & 281.1370 & 544.2402 & 272.6237 & 543.2562 & 272.1317 & $Q$ & 1427.6987 & 714.3530 & 1410.6722 & 705.8397 & 1409.6882 & 705.3477 & \begin{tabular}{l|l}
7 & 12
\end{tabular} \\
\hline 6 & 676.2937 & 338.6505 & 659.2671 & 330.1372 & 658.2831 & 329.6452 & D & 1299.6402 & 650.3237 & 1282.6136 & 641.8104 & 1281.6296 & 641.3184 & $\begin{array}{l}411 \\
11\end{array}$ \\
\hline 7 & 775.3621 & 388.1847 & 758.3355 & 379.6714 & 757.3515 & 379.1794 & V & 1184.6132 & 592.8102 & 1167.5867 & 584.2970 & 1166.6026 & 583.8050 & 10 \\
\hline 8 & 890.3890 & 445.6981 & 873.3625 & 437.1849 & 872.3785 & 436.6929 & D & 1085.5448 & 543.2760 & 1068.5183 & 534.7628 & 1067.5342 & 534.2708 & 9 \\
\hline 9 & 1021.4295 & 511.2184 & 1004.4029 & 502.7051 & 1003.4189 & 502.2131 & M & 970.5179 & 485.7626 & 953.4913 & 477.2493 & 952.5073 & 476.7573 & 8 \\
\hline 10 & 1120.4979 & 560.7526 & 1103.4714 & 552.2393 & 1102.4873 & 551.7473 & V & 839.4774 & 420.2423 & 822.4508 & 411.7291 & 821.4668 & 411.2370 & 7 \\
\hline 11 & 1267.5663 & 634.2868 & 1250.5398 & 625.7735 & 1249.5558 & 625.2815 & $\mathbf{F}$ & 740.4090 & 370.7081 & 723.3824 & 362.1948 & 722.3984 & 361.7028 & 6 \\
\hline 12 & 1338.6034 & 669.8054 & 1321.5769 & 661.2921 & 1320.5929 & 660.8001 & A & 593.3406 & 297.1739 & 576.3140 & 288.6606 & 575.3300 & 288.1686 & 5 \\
\hline 13 & 1425.6355 & 713.3214 & 1408.6089 & 704.8081 & 1407.6249 & 704.3161 & s & 522.3035 & 261.6554 & 505.2769 & 253.1421 & 504.2929 & 252.6501 & 4 \\
\hline 14 & 1572.7039 & 786.8556 & 1555.6773 & 778.3423 & 1554.6933 & 777.8503 & $\mathrm{~F}$ & 435.2714 & 218.1393 & 418.2449 & 209.6261 & & & 3 \\
\hline 15 & 1685.7879 & 843.3976 & 1668.7614 & 834.8843 & 1667.7774 & 834.3923 & I & 288.2030 & 144.6051 & 271.1765 & 136.0919 & & & 2 \\
\hline 16 & & & & & & & $\mathbf{R}$ & 175.1190 & 88.0631 & 158.0924 & 79.5498 & & & 1 \\
\hline
\end{tabular}
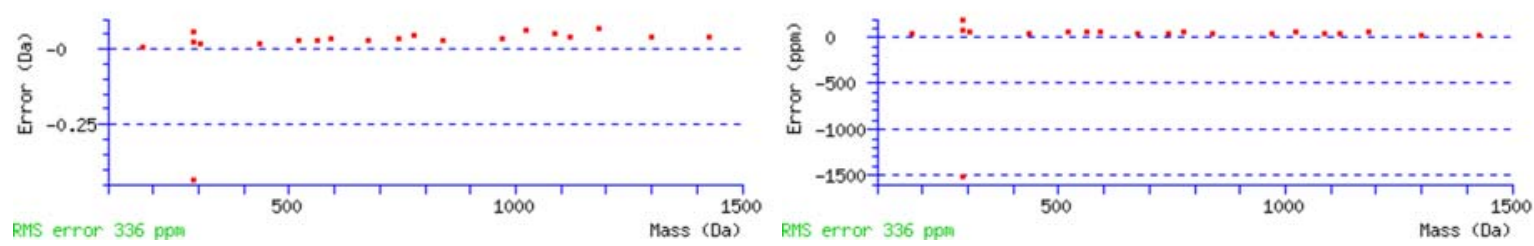


\section{Peptide View}

MS/MS Fragmentation of FIENLLASD GDFWIGLK

Found in gi|94385870|ref|XP_922372.2|, PREDICTED: similar to layilin [Mus musculus]

Match to Query 468: 1937.087048 from $\left(969.550800,2^{+}\right)$

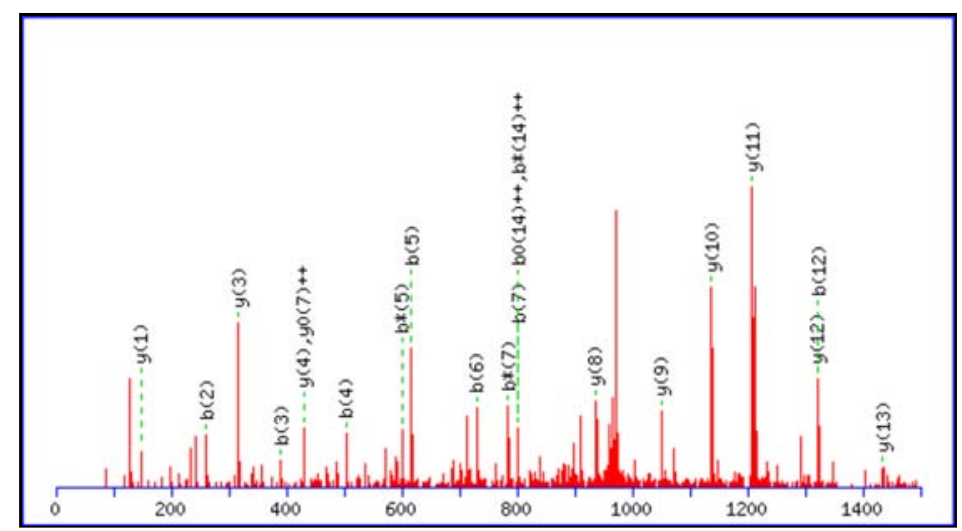

Monoisotopic mass of neutral peptide $\operatorname{Mr}$ (calc): 1936.9934

Ions Score: 53 Expect: 0.018

Matches (Bold Red) : 21/170 fragment ions using 52 most intense peaks

\begin{tabular}{|c|c|c|c|c|c|c|c|c|c|c|c|c|c|c|}
\hline \# & b & $b^{++}$ & b* & $b *^{++}$ & $b^{0}$ & $b^{0++}$ & Seq. & $\mathbf{y}$ & $y^{++}$ & y* & $y *^{++}$ & $y^{0}$ & $y^{0++}$ & $\#$ \\
\hline 1 & 148.0757 & 74.5415 & & & & & $\mathrm{~F}$ & & & & & & & 17 \\
\hline 2 & 261.1597 & 131.0835 & & & & & I & 1790.9323 & 895.9698 & 1773.9057 & 887.4565 & 1772.9217 & 886.9645 & 16 \\
\hline 3 & 390.2023 & 195.6048 & & & 372.1918 & 186.5995 & E & 1677.8482 & 839.4277 & 1660.8217 & 830.9145 & 1659.8377 & 830.4225 & 15 \\
\hline 4 & 504.2453 & 252.6263 & 487.2187 & 244.1130 & 486.2347 & 243.6210 & $\mathrm{~N}$ & 1548.8056 & 774.9065 & 1531.7791 & 766.3932 & 1530.7951 & 765.9012 & 14 \\
\hline 5 & 617.3293 & 309.1683 & 600.3028 & 300.6550 & 599.3188 & 300.1630 & L & 1434.7627 & 717.8850 & 1417.7362 & 709.3717 & 1416.7521 & \begin{tabular}{|l|}
708.8797 \\
\end{tabular} & 13 \\
\hline 6 & 730.4134 & 365.7103 & 713.3868 & 357.1971 & 712.4028 & 356.7050 & L & 1321.6786 & 661.3430 & 1304.6521 & 652.8297 & 1303.6681 & 652.3377 & 12 \\
\hline 7 & 801.4505 & 401.2289 & 784.4239 & 392.7156 & 783.4399 & 392.2236 & A & 1208.5946 & 604.8009 & 1191.5680 & 596.2877 & 1190.5840 & 595.7956 & 11 \\
\hline 8 & 888.4825 & 444.7449 & 871.4560 & 436.2316 & 870.4720 & 435.7396 & S & 1137.5575 & 569.2824 & 1120.5309 & 560.7691 & 1119.5469 & 560.2771 & 10 \\
\hline 9 & 1003.5095 & 502.2584 & 986.4829 & 493.7451 & 985.4989 & 493.2531 & D & 1050.5254 & 525.7664 & 1033.4989 & 517.2531 & 1032.5149 & 516.7611 & 9 \\
\hline 10 & 1060.5309 & 530.7691 & 1043.5044 & 522.2558 & 1042.5204 & 521.7638 & G & 935.4985 & 468.2529 & 918.4720 & 459.7396 & 917.4879 & 459.2476 & 8 \\
\hline 11 & 1175.5579 & 588.2826 & 1158.5313 & 579.7693 & 1157.5473 & 579.2773 & D & 878.4770 & 439.7422 & 861.4505 & 431.2289 & 860.4665 & 430.7369 & 7 \\
\hline 12 & 1322.6263 & 661.8168 & 1305.5997 & 653.3035 & 1304.6157 & 652.8115 & $F$ & 763.4501 & 382.2287 & 746.4236 & 373.7154 & & & 6 \\
\hline 13 & 1508.7056 & 754.8564 & 1491.6790 & 746.3432 & 1490.6950 & 745.8511 & W & 616.3817 & 308.6945 & 599.3551 & 300.1812 & & & 5 \\
\hline 14 & 1621.7896 & 811.3985 & 1604.7631 & 802.8852 & \begin{tabular}{|l|}
1603.7791 \\
\end{tabular} & 802.3932 & I & 430.3024 & 215.6548 & 413.2758 & 207.1416 & & & 4 \\
\hline 15 & 1678.8111 & 839.9092 & 1661.7846 & 831.3959 & 1660.8005 & 830.9039 & G & 317.2183 & 159.1128 & 300.1918 & 150.5995 & & & 3 \\
\hline 16 & 1791.8952 & 896.4512 & 1774.8686 & 887.9379 & 1773.8846 & 887.4459 & $L$ & 260.1969 & 130.6021 & 243.1703 & 122.0888 & & & 2 \\
\hline 17 & & & & & & & $\mathrm{~K}$ & 147.1128 & 74.0600 & 130.0863 & 65.5468 & & & 1 \\
\hline
\end{tabular}
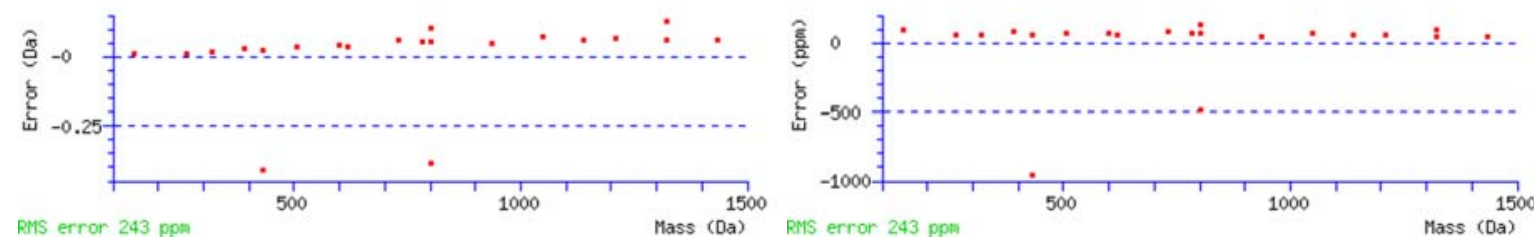


\section{Peptide View}

MS/MS Fragmentation of FINMFAVLDELK

Found in gi|84370256|ref|NP_598530.2|, cytoplasmic FMR1 interacting protein 2 [Mus musculus]

Match to Query 288: 1438.826848 from $(720.420700,2+)$

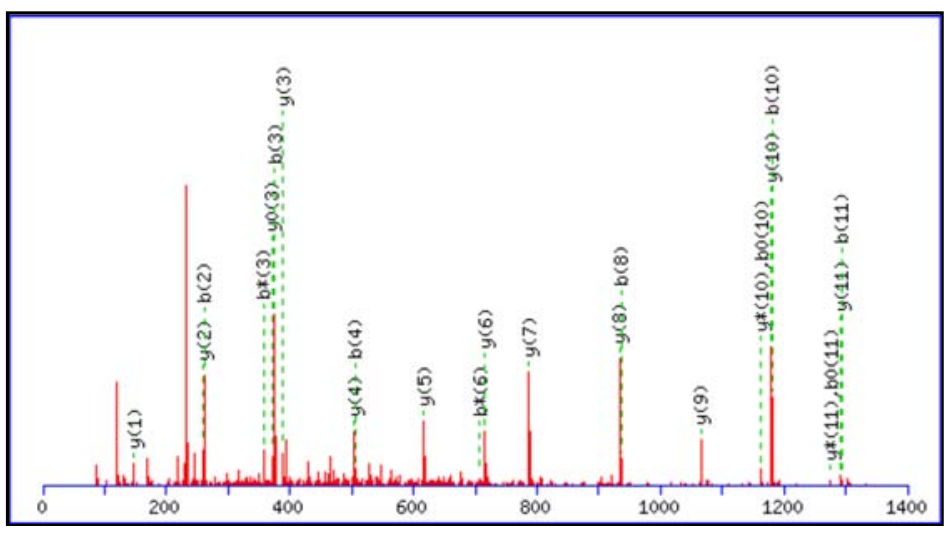

Monoisotopic mass of neutral peptide $\operatorname{Mr}(\mathrm{calc}): 1438.7530$

Ions Score: 65 Expect: 0.00052

Matches (Bold Red) : 24/108 fragment ions using 57 most intense peaks

\begin{tabular}{|c|c|c|c|c|c|c|c|c|c|c|c|c|c|c|}
\hline \# & b & $\mathrm{b}^{++}$ & b* & $\mathrm{b} *^{++}$ & $b^{0}$ & $b^{0++}$ & Seq. & y & $\mathrm{y}^{++}$ & y* & $y *^{++}$ & $y^{0}$ & $\mathrm{y}^{0++}$ & $\#$ \\
\hline 1 & 148.0757 & 74.5415 & & & & & $\mathrm{~F}$ & & & & & & & 12 \\
\hline 2 & 261.1597 & 131.0835 & & & & & 1 & 1292.6919 & 646.8496 & 1275.6653 & 638.3363 & 1274.6813 & 637.8443 & 11 \\
\hline 3 & 375.2027 & 188.1050 & 358.1761 & 179.5917 & & & $\mathrm{~N}$ & 1179.6078 & 590.3075 & 1162.5812 & 581.7943 & 1161.5972 & 581.3023 & 10 \\
\hline 4 & 506.2432 & 253.6252 & 489.2166 & 245.1119 & & & $M$ & 1065.5649 & 533.2861 & 1048.5383 & 524.7728 & 1047.5543 & 524.2808 & 9 \\
\hline 5 & 653.3116 & 327.1594 & 636.2850 & 318.6461 & & & $\mathrm{~F}$ & 934.5244 & 467.7658 & 17.4978 & 459.2526 & 916.5138 & 458.7605 & 8 \\
\hline 6 & 724.3487 & 362.6780 & 707.3221 & 354.1647 & & & A & 787.4560 & 394.2316 & 770.4294 & 385.7183 & 769.4454 & 385.2263 & 7 \\
\hline 7 & 823.4171 & 412.2122 & 806.3905 & 403.6989 & & & $\mathrm{~V}$ & 6.4189 & 358.7131 & 99.3923 & 350.1998 & 698.4083 & 349.7078 & 6 \\
\hline 8 & 936.5011 & 468.7542 & 919.4746 & 460.2409 & & & L & 617.3505 & 309.1789 & 600.3239 & 300.6656 & 599.3399 & 300.1736 & 5 \\
\hline 9 & 1051.5281 & 526.2677 & 1034.5015 & 517.7544 & 1033.5175 & 517.2624 & D & 664 & 252.6368 & 487.2398 & 244.1236 & 486.2558 & 243.6316 & 4 \\
\hline 10 & 1180.5707 & 590.7890 & 1163.5441 & 582.2757 & 1162.5601 & 581.7837 & $E$ & 389.2395 & 195.1234 & 372.2129 & 186.6101 & 371.2289 & 186.1181 & 3 \\
\hline 11 & 1293.6547 & 647.3310 & 1276.6282 & 638.8177 & 1275.6442 & 638.3257 & $\mathrm{~L}$ & 260.1969 & 130.6021 & 243.1703 & 122.0888 & & & 2 \\
\hline 12 & & & & & & & $\mathrm{~K}$ & 147.1128 & 74.0600 & 130.0863 & 65.5468 & & & 1 \\
\hline
\end{tabular}
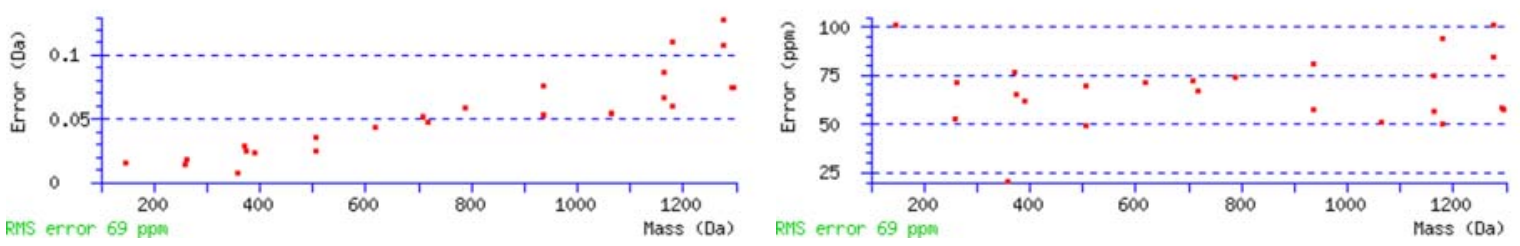


\section{Peptide View}

MS/MS Fragmentation of FLDGELPLDSFIDVYQSK

Found in gi|29244484|ref|NP_808544.1|, vacuolar protein sorting 37B [Mus musculus]

Match to Query 685: 2085.053848 from(1043.534200,2+)

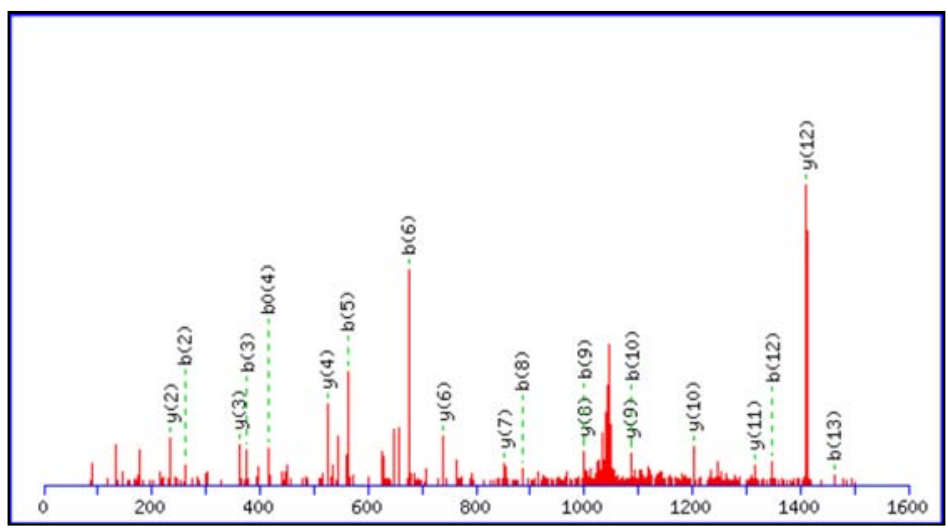

Monoisotopic mass of neutral peptide $\operatorname{Mr}(\mathrm{calc}): 2085.0306$

Ions Score: 57 Expect: 0.011

Matches (Bold Red): 20/168 fragment ions using 75 most intense peaks

\begin{tabular}{|c|c|c|c|c|c|c|c|c|c|c|c|c|c|c|}
\hline \# & b & $b^{++}$ & $\mathrm{b} *$ & $b *^{++}$ & $b^{0}$ & $b^{0++}$ & Seq. & $y$ & $y^{++}$ & y* & $y *^{++}$ & $y^{0}$ & $y^{0++}$ & $\#$ \\
\hline 1 & 148.0757 & 74.5415 & & & & & $F$ & & & & & & & 18 \\
\hline 2 & 261.1597 & 131.0835 & & & & & $\mathrm{~L}$ & 1938.9695 & 969.9884 & 1921.9429 & 961.4751 & 1920.9589 & 960.9831 & 17 \\
\hline 3 & 376.1867 & 188.5970 & & & 358.1761 & 179.5917 & D & 1825.8854 & 913.4463 & 1808.8589 & 904.9331 & 1807.8748 & 904.4411 & 16 \\
\hline 4 & 433.2081 & 217.1077 & & & 415.1976 & 208.1024 & $G$ & 1710.8585 & 855.9329 & 1693.8319 & 847.4196 & 1692.8479 & 846.9276 & 15 \\
\hline 5 & 562.2507 & 281.6290 & & & 544.2402 & 272.6237 & $\mathrm{E}$ & 1653.8370 & 827.4221 & 1636.8105 & 818.9089 & 1635.8264 & 818.4169 & 14 \\
\hline 6 & 675.3348 & 338.1710 & & & 657.3242 & 329.1658 & L & 1524.7944 & 762.9008 & 1507.7679 & 754.3876 & 1506.7838 & 753.8956 & 13 \\
\hline 7 & 772.3876 & 386.6974 & & & 754.3770 & 377.6921 & $\mathrm{P}$ & 1411.7104 & 706.3588 & 1394.6838 & 697.8455 & 1393.6998 & 697.3535 & 12 \\
\hline 8 & 885.4716 & 443.2394 & & & 867.4611 & 434.2342 & L & 1314.6576 & 657.8324 & 1297.6310 & 649.3192 & 1296.6470 & 648.8272 & 11 \\
\hline 9 & 1000.4986 & 500.7529 & & & 982.4880 & 491.7476 & D & 1201.5735 & 601.2904 & 1184.5470 & 592.7771 & 1183.5630 & 592.2851 & 10 \\
\hline 10 & 1087.5306 & 544.2689 & & & 1069.5200 & 535.2636 & $S$ & 1086.5466 & 543.7769 & 1069.5200 & 535.2637 & 1068.5360 & 534.7717 & 9 \\
\hline 11 & 1234.5990 & 617.8031 & & & 1216.5884 & 608.7979 & $F$ & 999.5146 & 500.2609 & 982.4880 & 491.7476 & 981.5040 & 491.2556 & 8 \\
\hline 12 & 1347.6831 & 674.3452 & & & 1329.6725 & 665.3399 & I & 852.4462 & 426.7267 & 835.4196 & 418.2134 & 834.4356 & 417.7214 & 7 \\
\hline 13 & 1462.7100 & 731.8586 & & & 1444.6994 & 722.8534 & D & 739.3621 & 370.1847 & 722.3355 & 361.6714 & 721.3515 & 361.1794 & 6 \\
\hline 14 & 1561.7784 & 781.3928 & & & 1543.7678 & 772.3876 & V & 624.3352 & 312.6712 & 607.3086 & 304.1579 & 606.3246 & 303.6659 & 5 \\
\hline 15 & 1724.8417 & 862.9245 & & & 1706.8312 & 853.9192 & $\mathrm{Y}$ & 525.2667 & 263.1370 & 508.2402 & 254.6237 & 507.2562 & 254.1317 & 4 \\
\hline 16 & 1852.9003 & 926.9538 & 1835.8738 & 918.4405 & 1834.8898 & 917.9485 & $Q$ & 362.2034 & 181.6053 & 345.1769 & 173.0921 & 344.1928 & 172.6001 & 3 \\
\hline 17 & 1939.9323 & 970.4698 & 1922.9058 & 961.9565 & 1921.9218 & 961.4645 & $S$ & 234.1448 & 117.5761 & 217.1183 & 109.0628 & 216.1343 & 108.5708 & 2 \\
\hline 18 & & & & & & & $\mathrm{~K}$ & 147.1128 & 74.0600 & 130.0863 & 65.5468 & & & 1 \\
\hline
\end{tabular}
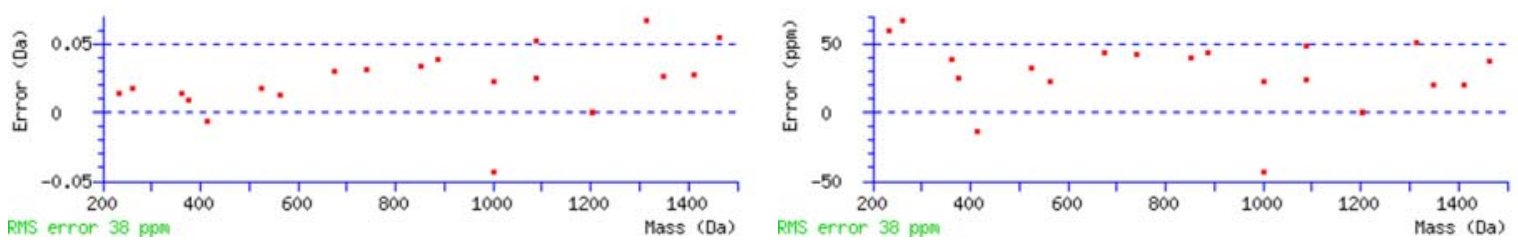


\section{Peptide View}

MS/MS Fragmentation of FYTNPSYFFDLWK

Found in gi|13994209|ref|NP_114083.1|, WASP family 1 [Mus musculus]

Match to Query 650: 1726.949048 from(864.481800,2+)

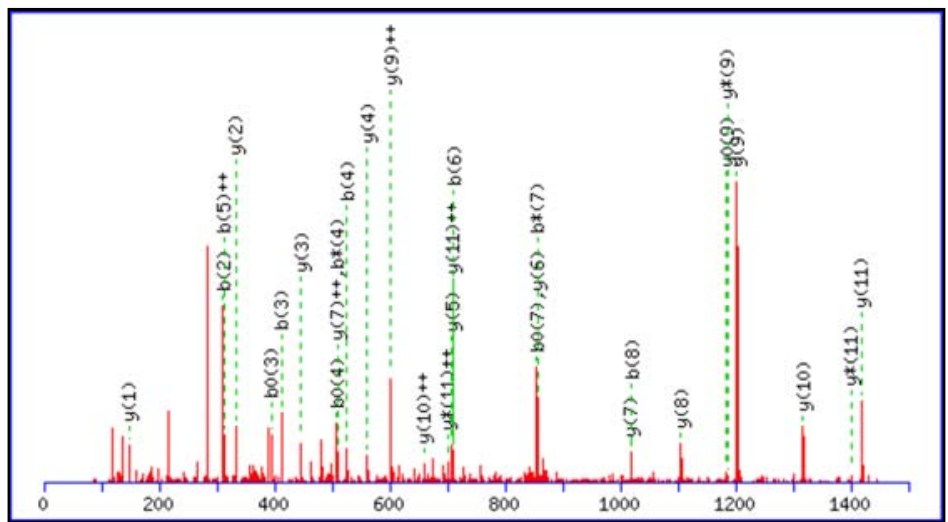

Monoisotopic mass of neutral peptide $\mathrm{Mr}$ (calc): 1726.8031

Ions Score: 49 Expect: 0.032

Matches (Bold Red): 30/128 fragment ions using 77 most intense peaks

\begin{tabular}{|c|c|c|c|c|c|c|c|c|c|c|c|c|c|c|}
\hline$\#$ & b & $\mathrm{b}^{++}$ & b* & $\mathrm{b} *^{++}$ & $b^{0}$ & $b^{0++}$ & Seq. & $y$ & $y^{++}$ & y* & $y *^{++}$ & $y^{0}$ & $y^{0++}$ & $\#$ \\
\hline 1 & 148.0757 & 74.5415 & & & & & $F$ & & & & & & & 13 \\
\hline 2 & 311.1390 & 156.0731 & & & & & $Y$ & 1580.7420 & 790.8746 & 1563.7154 & 782.3614 & 1562.7314 & 781.8694 & 12 \\
\hline 3 & 412.1867 & 206.5970 & & & 394.1761 & 197.5917 & $\mathrm{~T}$ & 1417.6787 & 709.3430 & 1400.6521 & 700.8297 & 1399.6681 & 700.3377 & 11 \\
\hline 4 & 526.2296 & 263.6185 & 509.2031 & 255.1052 & 508.2191 & 254.6132 & $\mathrm{~N}$ & 1316.6310 & 658.8191 & 1299.6044 & 650.3059 & 1298.6204 & 649.8138 & 10 \\
\hline 5 & 623.2824 & 312.1448 & 606.2558 & 316 & 605.2718 & 303.1395 & $P$ & 1202.5881 & 601.7977 & 1185.5615 & 593.2844 & 1184.5775 & 592.7924 & 9 \\
\hline 6 & 710.3144 & 355.6608 & 693.2879 & 347.1476 & 692.3039 & 346.6556 & S & 1105.5353 & 553.2713 & 1088.5087 & 544.7580 & 1087.5247 & 544.2660 & 8 \\
\hline 7 & 873.3777 & 437.1925 & 856.3512 & 428.6792 & 855.3672 & 428.1872 & $Y$ & 1018.5033 & 509.7553 & 1001.4767 & 501.2420 & 1000.4927 & 500.7500 & 7 \\
\hline 8 & 1020.4462 & 510.7267 & 1003.4196 & 502.2134 & 1002.4356 & 501.7214 & $F$ & 855.4399 & 428.2236 & 838.4134 & 419.7103 & 837.4294 & 419.2183 & 6 \\
\hline 9 & 1167.5146 & 584.2609 & 1150.4880 & 575.7476 & 1149.5040 & 575.2556 & $F$ & 708.3715 & 354.6894 & 691.3450 & 346.1761 & 690.3610 & 345.6841 & 5 \\
\hline 10 & 1282.5415 & 641.7744 & 1265.5150 & 633.2611 & 1264.5309 & 632.7691 & D & 561.3031 & 281.1552 & 544.2766 & 272.6419 & 543.2925 & 272.1499 & 4 \\
\hline 11 & 1395.6256 & 698.3164 & 1378.5990 & 689.8031 & 1377.6150 & 689.3111 & L & 446.2762 & 223.6417 & 429.2496 & 215.1284 & & & 3 \\
\hline 12 & 1581.7049 & 791.3561 & 1564.6783 & 782.8428 & 1563.6943 & 782.3508 & W & 333.1921 & 167.0997 & 316.1656 & 158.5864 & & & 2 \\
\hline 13 & & & & & & & $\mathrm{~K}$ & 147.1128 & 74.0600 & 130.0863 & 65.5468 & & & 1 \\
\hline
\end{tabular}
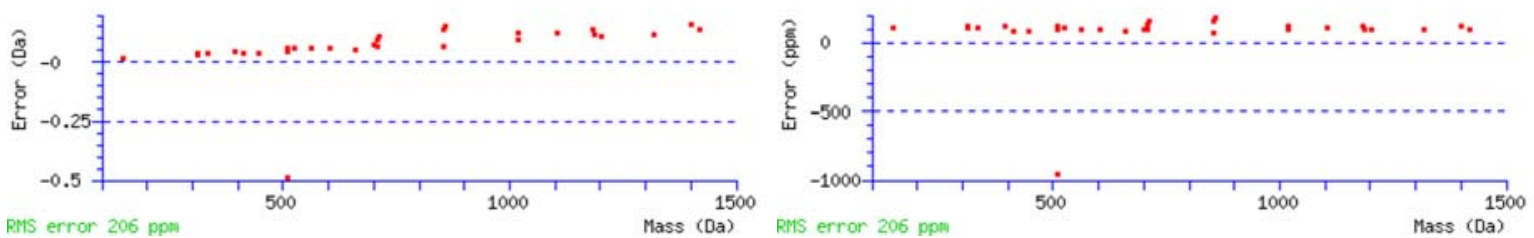

RMS error 206 ppon

Mass (Da)

ass (Da) 


\section{Peptide View}

MS/MS Fragmentation of GLTVVQAFQELVEPK

Found in gi|19882207|ref|NP_608219.1|, farnesyl diphosphate synthetase [Mus musculus]

Match to Query 381: 1656.988248 from $\left(829.501400,2^{+}\right)$

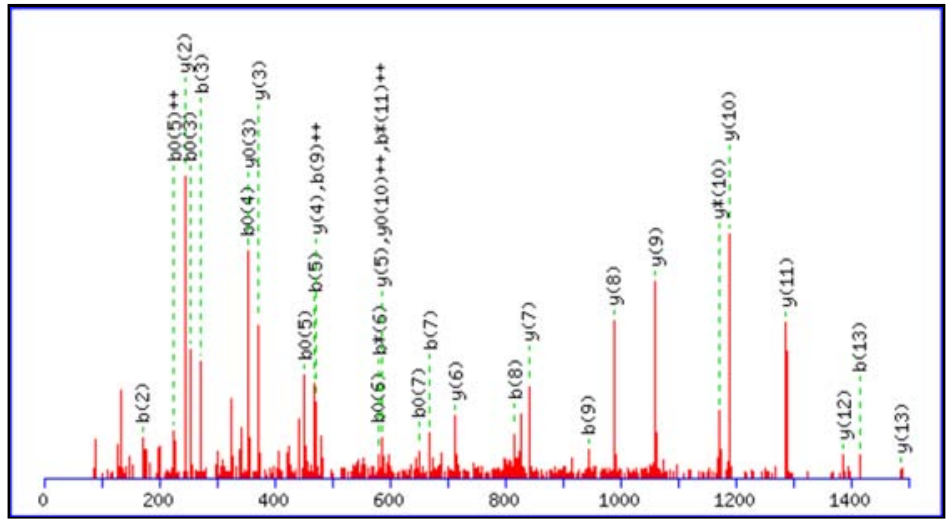

Monoisotopic mass of neutral peptide $\operatorname{Mr}(\mathrm{calc}): 1656.9086$

Ions Score: 88 Expect: $4 \mathrm{e}-006$

Matches (Bold Red): 31/150 fragment ions using 49 most intense peaks

\begin{tabular}{|c|c|c|c|c|c|c|c|c|c|c|c|c|c|c|}
\hline \# & b & $b^{++}$ & $b *$ & $\mathrm{~b} *^{++}$ & $b^{0}$ & $b^{0++}$ & Seq. & $y$ & $y^{++}$ & y* & $\mathrm{y}^{++}$ & $y^{0}$ & $y^{0++}$ & $\#$ \\
\hline 1 & 58.0287 & 29.5180 & & & & & G & & & & & & & 15 \\
\hline 2 & 171.1128 & 86.0600 & & & & & L & 1600.8945 & 800.9509 & 1583.8679 & 792.4376 & 1582.8839 & 791.9456 & 14 \\
\hline 3 & 272.1605 & 136.5839 & & & 254.1499 & $\mid 127.5786$ & $T$ & 1487.8104 & 744.4088 & 1470.7838 & 735.8956 & 1469.7998 & 735.4036 & 13 \\
\hline 4 & 371.2289 & 186.1181 & & & 353.2183 & \begin{tabular}{|c|}
177.1128 \\
\end{tabular} & V & 1386.7627 & 693.8850 & 1369.7362 & 685.3717 & 1368.7521 & 684.8797 & 12 \\
\hline 5 & 470.2973 & 235.6523 & & & 452.2867 & 226.6470 & V & 1287.6943 & 644.3508 & 1270.6678 & 635.8375 & 1269.6837 & 635.3455 & 11 \\
\hline 6 & 598.3559 & 299.6816 & 581.3293 & 291.1683 & 580.3453 & 290.6763 & $Q$ & 1188.6259 & 594.8166 & 1171.5993 & 586.3033 & 1170.6153 & 585.8113 & 10 \\
\hline 7 & 669.3930 & 335.2001 & 652.3664 & 326.6869 & 651.3824 & 326.1948 & A & 1060.5673 & 530.7873 & 1043.5408 & 522.2740 & 1042.5567 & 521.7820 & 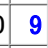 \\
\hline 8 & 816.4614 & 408.7343 & 799.4348 & 400.2211 & \begin{tabular}{|l|}
798.4508 \\
\end{tabular} & 399.7291 & $\mathrm{~F}$ & 989.5302 & 495.2687 & 972.5037 & 486.7555 & 971.5196 & 486.2635 & 8 \\
\hline 9 & 944.5200 & 472.7636 & 927.4934 & 464.2504 & 926.5094 & 463.7583 & $Q$ & 842.4618 & 421.7345 & 825.4352 & 413.2213 & 824.4512 & 412.7293 & 3 \\
\hline 10 & 1073.5626 & 537.2849 & 1056.5360 & 528.7716 & 1055.5520 & 528.2796 & $E$ & 714.4032 & 357.7052 & 697.3767 & 349.1920 & 696.3926 & 348.7000 & 6 \\
\hline 11 & 1186.6466 & 593.8270 & 1169.6201 & 585.3137 & 1168.6361 & 584.8217 & L & 585.3606 & 293.1839 & 568.3341 & 284.6707 & 567.3501 & 284.1787 & 5 \\
\hline 12 & 1285.7150 & 643.3612 & 1268.6885 & 634.8479 & 1267.7045 & 634.3559 & V & 472.2766 & 236.6419 & 455.2500 & 228.1286 & 454.2660 & 227.6366 & 4 \\
\hline 13 & 1414.7576 & 707.8825 & 1397.7311 & 699.3692 & 1396.7471 & 698.8772 & $E$ & 373.2082 & 187.1077 & 356.1816 & 178.5944 & 355.1976 & 178.1024 & $t$ \\
\hline 14 & 1511.8104 & 756.4088 & 1494.7838 & 747.8956 & 1493.7998 & 747.4035 & $P$ & 244.1656 & 122.5864 & 227.1390 & 114.0731 & & & 2 \\
\hline 15 & & & & & & & K & 147.1128 & 74.0600 & 130.0863 & 65.5468 & & & 1 \\
\hline
\end{tabular}

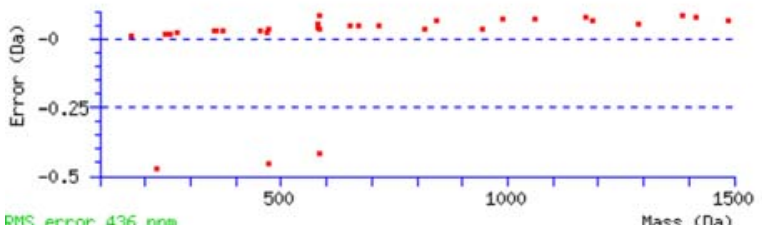

RMS error 436 ppm

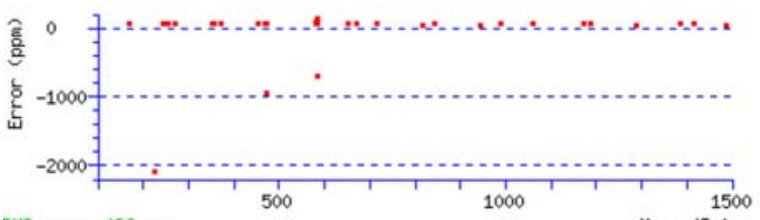

Mass (Da) 


\section{Peptide View}

MS/MS Fragmentation of GMGGAFVLVLYDEIK

Found in gi|22094075|ref|NP_031477.1|, solute carrier family 25, member 5 [Mus musculus]

Match to Query 362: 1610.915648 from(806.465100,2+)

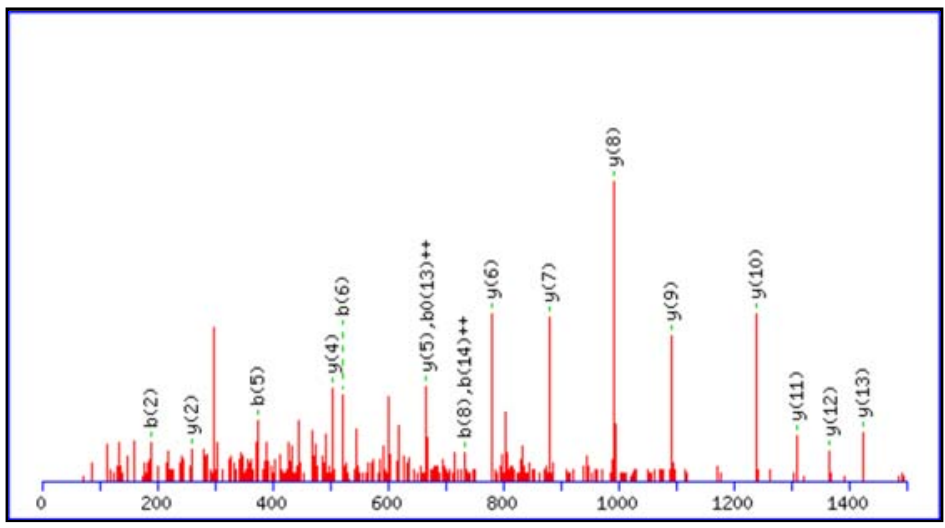

Monoisotopic mass of neutral peptide $\operatorname{Mr}$ (calc): 1610.8377

Ions Score: 73 Expect: 0.00011

Matches (Bold Red) : 17/114 fragment ions using 32 most intense peaks

\begin{tabular}{|c|c|c|c|c|c|c|c|c|c|c|c|c|}
\hline \# & b & $\mathrm{b}^{++}$ & $b^{0}$ & $b^{0++}$ & Seq. & $\mathbf{y}$ & $y^{++}$ & y* & $y *^{++}$ & $y^{0}$ & $y^{0++}$ & $\#$ \\
\hline 1 & 58.0287 & 29.5180 & & & G & & & & & & & 15 \\
\hline 2 & 189.0692 & 95.0382 & & & M & 1554.8236 & 777.9154 & 1537.7970 & 769.4022 & 1536.8130 & 768.9101 & 14 \\
\hline 3 & 246.0907 & 123.5490 & & & G & 1423.7831 & 712.3952 & 1406.7566 & 703.8819 & 1405.7725 & 703.3899 & 13 \\
\hline 4 & 303.1121 & 152.0597 & & & G & 1366.7616 & 683.8845 & 1349.7351 & 675.3712 & 1348.7511 & 674.8792 & 12 \\
\hline 5 & 374.1492 & 187.5783 & & & A & 1309.7402 & 655.3737 & 1292.7136 & 646.8605 & 1291.7296 & 646.3684 & 11 \\
\hline 6 & 521.2177 & 261.1125 & & & $\mathrm{~F}$ & 1238.7031 & 619.8552 & 1221.6765 & 611.3419 & 1220.6925 & 610.8499 & 10 \\
\hline 7 & 620.2861 & 310.6467 & & & V & 1091.6347 & 546.3210 & 1074.6081 & 537.8077 & 1073.6241 & 537.3157 & 9 \\
\hline 8 & 733.3701 & 367.1887 & & & L & 992.5663 & 496.7868 & 975.5397 & 488.2735 & 974.5557 & 487.7815 & 8 \\
\hline 9 & 832.4385 & 416.7229 & & & V & 879.4822 & 440.2447 & 862.4556 & 431.7315 & 861.4716 & 431.2395 & 7 \\
\hline 10 & 945.5226 & 473.2649 & & & $L$ & 780.4138 & 390.7105 & 763.3872 & 382.1973 & 762.4032 & 381.7052 & 6 \\
\hline 11 & 1108.5859 & 554.7966 & & & $Y$ & 667.3297 & 334.1685 & 650.3032 & 325.6552 & 649.3192 & 325.1632 & 5 \\
\hline 12 & 1223.6129 & 612.3101 & 1205.6023 & 603.3048 & D & 504.2664 & 252.6368 & 487.2398 & 244.1236 & 486.2558 & 243.6316 & 4 \\
\hline 13 & 1352.6555 & 676.8314 & 1334.6449 & 667.8261 & E & 389.2395 & 195.1234 & 372.2129 & 186.6101 & 371.2289 & 186.1181 & 3 \\
\hline 14 & 1465.7395 & 733.3734 & 1447.7290 & 724.3681 & I & 260.1969 & 130.6021 & 243.1703 & 122.0888 & & & 2 \\
\hline 15 & & & & & $\mathrm{~K}$ & 147.1128 & 74.0600 & 130.0863 & 65.5468 & & & 1 \\
\hline
\end{tabular}
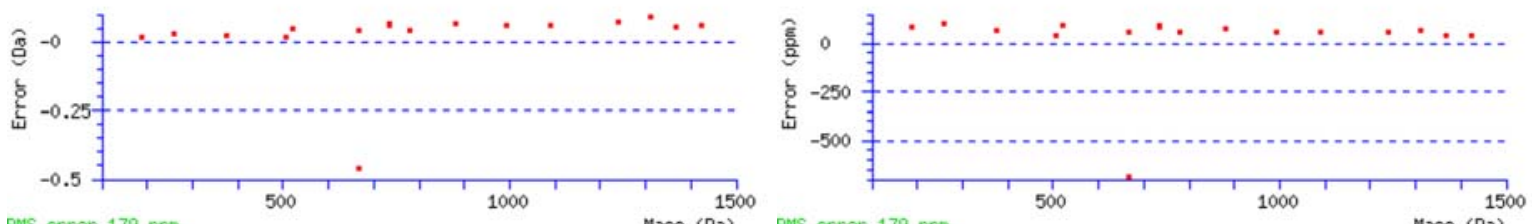

RMS error $179 \mathrm{ppm}$

Mass (Da) RMS error 179 ppm

Mass (Da) 


\section{Peptide View}

MS/MS Fragmentation of GSLDTMLGLLQSDLSR

Found in gi|6678313|ref|NP_033391.1|, transforming growth factor beta 1 induced transcript 1 [Mus musculus]

Match to Query 581: 1714.903448 from(858.459000,2+)

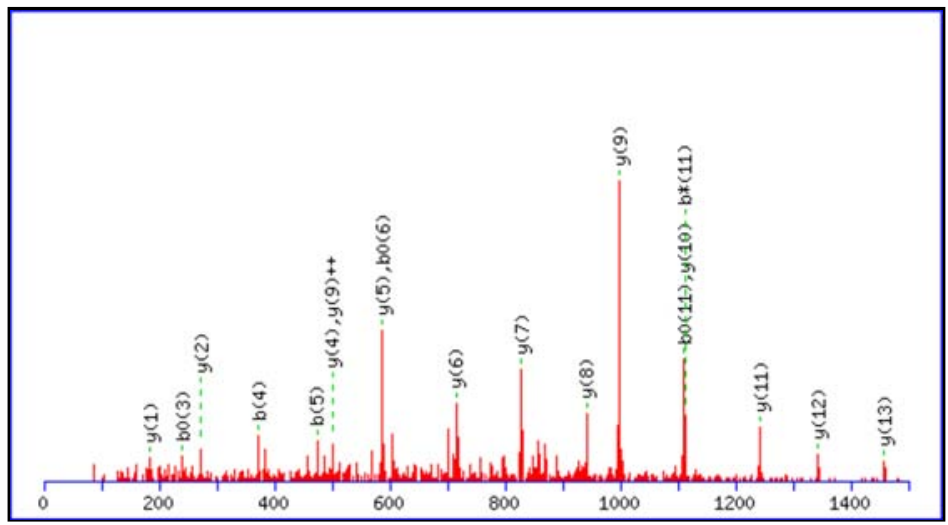

Monoisotopic mass of neutral peptide $\operatorname{Mr}(\mathrm{calc}): 1714.9052$

Variable modifications:

R16 : Arginine-13C615N4 (R-full)

Ions Score: 135 Expect: $8.7 \mathrm{e}-011$

Matches (Bold Red): 19/156 fragment ions using 17 most intense peaks

\begin{tabular}{|c|c|c|c|c|c|c|c|c|c|c|c|c|c|c|}
\hline$\#$ & b & $\mathrm{b}^{++}$ & b* & $b *^{++}$ & $b^{0}$ & $b^{0++}$ & Seq. & $y$ & $y^{++}$ & y* & $y *^{++}$ & $y^{0}$ & $y^{0++}$ & $\#$ \\
\hline 1 & 58.0287 & 29.5180 & & & & & G & & & & & & & 16 \\
\hline 2 & 145.0608 & 73.0340 & & & 127.0502 & 64.0287 & s & 1658.8910 & 829.9491 & 1641.8644 & 821.4359 & 3804 & 820.9438 & 15 \\
\hline 3 & 258.1448 & 129.5761 & & & 240.1343 & 120.5708 & L & 1571.8589 & 786.4331 & 1554.8324 & 777.9198 & 1553.8484 & 777.4278 & 14 \\
\hline 4 & 373.1718 & 187.0895 & & & 355.1612 & 178.0842 & D & 1458.7749 & 729.8911 & 1441.7483 & 721.3778 & 1440.7643 & 720.8858 & 13 \\
\hline 5 & 474.2194 & 237.6134 & & & 456.2089 & 228.6081 & $\mathrm{~T}$ & 1343.7479 & 672.3776 & 1326.7214 & 663.8643 & 1325.7374 & 663.3723 & 12 \\
\hline 6 & 605.2599 & 303.1336 & & & 587.2494 & 294.1283 & M & 1242.7003 & 621.8538 & 1225.6737 & 613.3405 & 1224.6897 & 612.8485 & 11 \\
\hline 7 & 718.3440 & 359.6756 & & & 700.3334 & 350.6703 & L & 1111.6598 & 556.3335 & 1094.6332 & 547.8203 & 1093.6492 & 547.3282 & 10 \\
\hline 8 & 775.3654 & 388.1864 & & & 757.3549 & 379.1811 & G & 998.5757 & 499.7915 & 981.5492 & 491.2782 & 980.5652 & 490.7862 & 9 \\
\hline 9 & 888.4495 & 444.7284 & & & 870.4389 & 435.7231 & L & 941.5543 & 471.2808 & 924.5277 & 462.7675 & 923.5437 & 462.2755 & 8 \\
\hline 10 & 1001.5336 & 501.2704 & & & 983.5230 & 492.2651 & L & 828.4702 & 414.7387 & 811.4437 & 406.2255 & 810.4596 & 405.7335 & 7 \\
\hline 11 & 1129.5921 & 565.2997 & 1112.5656 & 556.7864 & 1111.5816 & 556.2944 & $Q$ & 715.3861 & 358.1967 & 698.3596 & 349.6834 & 697.3756 & 349.1914 & 6 \\
\hline 12 & 1216.6242 & 608.8157 & 1199.5976 & 600.3025 & 1198.6136 & 599.8104 & $S$ & 587.3276 & 294.1674 & 570.3010 & 285.6541 & 569.3170 & 285.1621 & 5 \\
\hline 13 & 1331.6511 & 666.3292 & 1314.6246 & 657.8159 & 1313.6406 & 657.3239 & D & 500.2955 & 250.6514 & 483.2690 & 242.1381 & 482.2850 & 241.6461 & 4 \\
\hline 14 & 1444.7352 & 722.8712 & 1427.7086 & 714.3580 & 1426.7246 & 713.8659 & $\mathrm{~L}$ & 385.2686 & 193.1379 & 368.2420 & 184.6247 & 367.2580 & 184.1327 & 3 \\
\hline 15 & 1531.7672 & 766.3872 & 1514.7407 & 757.8740 & 1513.7566 & 757.3820 & $S$ & 272.1845 & 136.5959 & 255.1580 & 128.0826 & 254.1740 & 127.5906 & 2 \\
\hline 16 & & & & & & & $\mathrm{R}$ & 185.1525 & 93.0799 & 168.1260 & 84.5666 & & & 1 \\
\hline
\end{tabular}
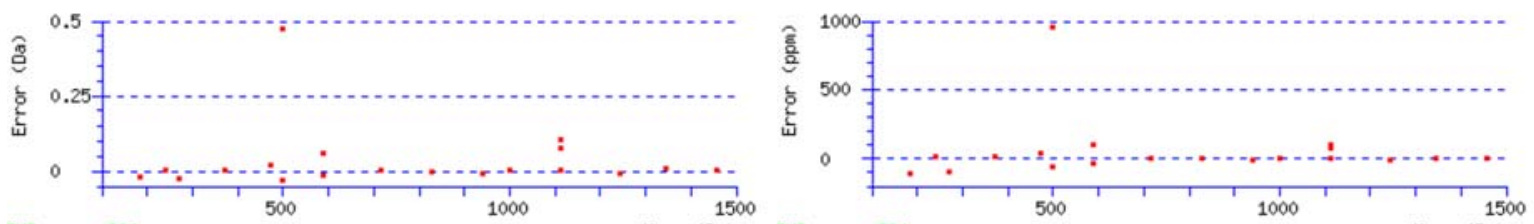

RMS error 224 ppes

Mass (Da)

RMS error 224 ppon

Mass (Da) 


\section{Peptide View}

MS/MS Fragmentation of GVFVSAFFSLLQTLSVK

Found in gi|31982724|ref|NP_058056.2|, MYB binding protein (P160) 1a [Mus musculus]

Match to Query 1035: 1842.164848 from $(922.089700,2+)$

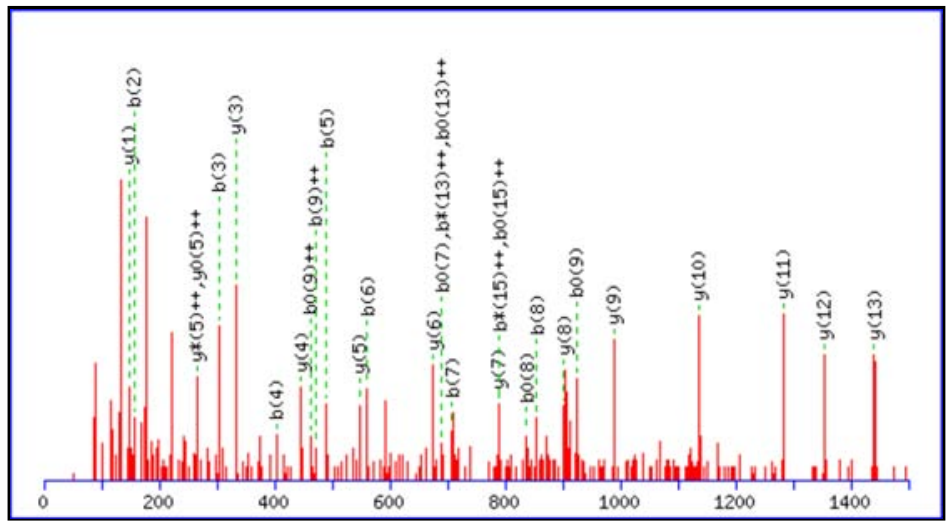

Monoisotopic mass of neutral peptide $\operatorname{Mr}(\mathrm{calc}): 1842.0290$

Ions Score: 51 Expect: 0.034

Matches (Bold Red): 30/158 fragment ions using 79 most intense peaks

\begin{tabular}{|c|c|c|c|c|c|c|c|c|c|c|c|c|c|c|}
\hline \# & b & $b^{++}$ & $b *$ & $\mathrm{~b} *^{++}$ & $b^{0}$ & $b^{0++}$ & Seq. & y & $y^{++}$ & y* & $y *^{++}$ & $y^{0}$ & $y^{0++}$ & $\#$ \\
\hline 1 & 58.0287 & 29.5180 & & & & & $G$ & & & & & & & 17 \\
\hline 2 & 157.0971 & 79.0522 & & & & & V & 1786.0149 & 893.5111 & 1768.9884 & 884.9978 & 1768.0043 & 884.5058 & 16 \\
\hline 3 & 304.1656 & 152.5864 & & & & & $\mathrm{~F}$ & 1686.9465 & 843.9769 & 1669.9199 & 835.4636 & 1668.9359 & 834.9716 & 15 \\
\hline 4 & 403.2340 & 202.1206 & & & & & V & 1539.8781 & 770.4427 & 1522.8515 & 761.9294 & 1521.8675 & 761.4374 & 14 \\
\hline 5 & 490.2660 & 245.6366 & & & 472.2554 & 236.6314 & S & 1440.8097 & 720.9085 & 1423.7831 & 712.3952 & 1422.7991 & 711.9032 & 13 \\
\hline 6 & 561.3031 & 281.1552 & & & 543.2925 & 272.1499 & A & 1353.7776 & 677.3925 & 1336.7511 & 668.8792 & 1335.7671 & 668.3872 & 12 \\
\hline 7 & 708.3715 & 354.6894 & & & 690.3610 & 345.6841 & $\mathrm{~F}$ & 1282.7405 & 641.8739 & 1265.7140 & 633.3606 & 1264.7300 & \begin{tabular}{|l|}
632.8686 \\
\end{tabular} & 11 \\
\hline 8 & 855.4399 & 428.2236 & & & 837.4294 & 419.2183 & $\mathrm{~F}$ & 1135.6721 & 568.3397 & 1118.6456 & 559.8264 & 1117.6616 & 559.3344 & 10 \\
\hline 9 & 942.4720 & 471.7396 & & & 924.4614 & 462.7343 & $\mathrm{~s}$ & 988.6037 & 494.8055 & 971.5772 & 486.2922 & 970.5931 & 485.8002 & 9 \\
\hline 10 & 1055.5560 & 528.2816 & & & 1037.5455 & 519.2764 & $\mathrm{~L}$ & 901.5717 & 451.2895 & 884.5451 & 442.7762 & 883.5611 & 442.2842 & 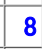 \\
\hline 11 & 1168.6401 & 584.8237 & & & 1150.6295 & 575.8184 & L & 788.4876 & 394.7474 & 771.4611 & 386.2342 & 770.4771 & 385.7422 & 7 \\
\hline 12 & 1296.6987 & 648.8530 & 1279.6721 & 640.3397 & 1278.6881 & 639.8477 & $Q$ & 675.4036 & 338.2054 & 658.3770 & 329.6921 & 657.3930 & 329.2001 & 6 \\
\hline 13 & 1397.7463 & 699.3768 & 1380.7198 & 690.8635 & 1379.7358 & 690.3715 & $T$ & 547.3450 & 274.1761 & 530.3184 & 265.6629 & 529.3344 & 265.1708 & 5 \\
\hline 14 & 1510.8304 & 755.9188 & 1493.8038 & 747.4056 & 1492.8198 & 746.9136 & $\mathrm{~L}$ & 446.2973 & 223.6523 & 429.2708 & 215.1390 & 428.2867 & 214.6470 & 4 \\
\hline 15 & 1597.8624 & 799.4349 & 1580.8359 & 790.9216 & 1579.8519 & 790.4296 & $S$ & 333.2132 & 167.1103 & 316.1867 & 158.5970 & 315.2027 & 158.1050 & 3 \\
\hline 16 & 1696.9308 & 848.9691 & 1679.9043 & 840.4558 & 1678.9203 & 839.9638 & V & 246.1812 & 123.5942 & 229.1547 & 115.0810 & & & 2 \\
\hline 17 & & & & & & & $\mathrm{~K}$ & 147.1128 & 74.0600 & 130.0863 & 65.5468 & & & 1 \\
\hline
\end{tabular}
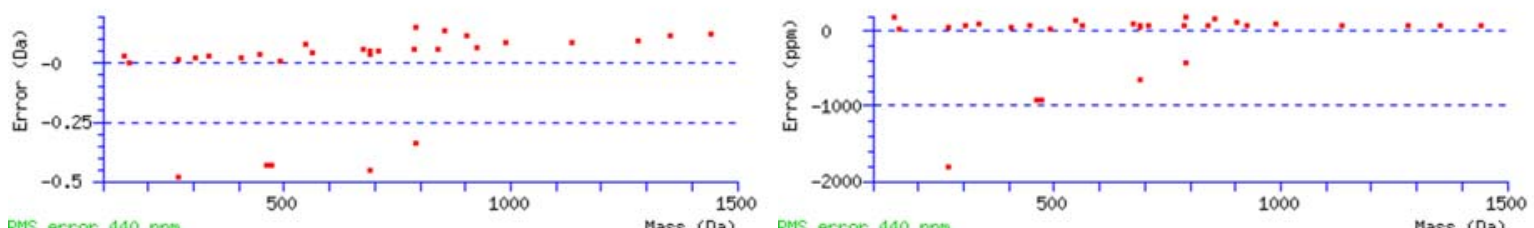

RMS error $440 \mathrm{ppm}$

Mass (Da) RMS error $440 \mathrm{ppm}$

Mass (Da) 


\section{Peptide View}

MS/MS Fragmentation of HALIIYDDLSK

Found in gi|6680748|ref|NP_031531.1|, ATP synthase, $\mathrm{H}^{+}$transporting, mitochondrial F1 complex, alpha subunit, isoform 1 [Mus musculus]

Match to Query 316: 1286.680248 from(644.347400,2+)

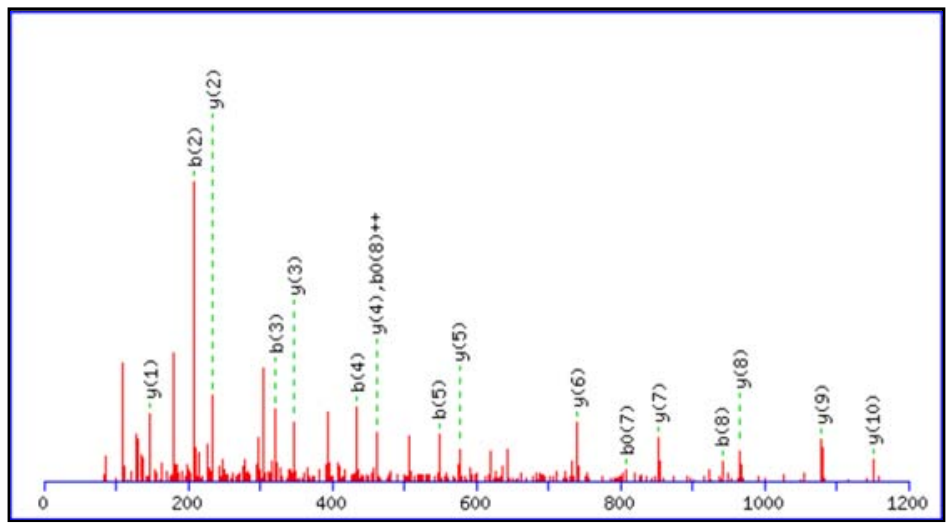

Monoisotopic mass of neutral peptide $\operatorname{Mr}(\mathrm{calc}): 1286.6870$

Ions Score: 70 Expect: 0.00013

Matches (Bold Red): 17/86 fragment ions using 33 most intense peaks

\begin{tabular}{|r|c|c|c|c|c|c|c|c|c|c|c|c|}
\hline$\#$ & $\mathbf{b}$ & $\mathbf{b}^{++}$ & $\mathbf{b}^{\mathbf{0}}$ & $\mathbf{b}^{\mathbf{0 + +}}$ & $\mathbf{S e q}$ & $\mathbf{y}$ & $\mathbf{y}^{++}$ & $\mathbf{y *}$ & $\mathbf{y}^{++}$ & $\mathbf{y}^{\mathbf{0}}$ & $\mathbf{y}^{\mathbf{0 + +}}$ & $\#$ \\
\hline $\mathbf{1}$ & $\mathbf{1 3 8 . 0 6 6 2}$ & 69.5367 & & & $\mathbf{H}$ & & & & & & & $\mathbf{1 1}$ \\
\hline $\mathbf{2}$ & 209.1033 & 105.0553 & & & A & 1150.6354 & 575.8213 & 1133.6088 & 567.3081 & 1132.6248 & 566.8161 & 10 \\
\hline $\mathbf{3}$ & 322.1874 & 161.5973 & & & L & 1079.5983 & 540.3028 & 1062.5717 & 531.7895 & 1061.5877 & 531.2975 & $\mathbf{9}$ \\
\hline $\mathbf{4}$ & 435.2714 & 218.1393 & & & I & 966.5142 & 483.7607 & 949.4877 & 475.2475 & 948.5037 & 474.7555 & $\mathbf{8}$ \\
\hline $\mathbf{5}$ & 548.3555 & 274.6814 & & & I & 853.4302 & 427.2187 & 836.4036 & 418.7054 & 835.4196 & 418.2134 & $\mathbf{7}$ \\
\hline $\mathbf{6}$ & 711.4188 & 356.2130 & & & Y & 740.3461 & 370.6767 & 723.3196 & 362.1634 & 722.3355 & 361.6714 & $\mathbf{6}$ \\
\hline $\mathbf{7}$ & 826.4457 & 413.7265 & 808.4352 & 404.7212 & D & 577.2828 & 289.1450 & 560.2562 & 280.6317 & 559.2722 & 280.1397 & $\mathbf{5}$ \\
\hline $\mathbf{8}$ & 941.4727 & 471.2400 & 923.4621 & 462.2347 & D & 462.2558 & 231.6316 & 445.2293 & 223.1183 & 444.2453 & 222.6263 & $\mathbf{4}$ \\
\hline $\mathbf{9}$ & 1054.5567 & 527.7820 & 1036.5462 & 518.7767 & L & 347.2289 & 174.1181 & 330.2023 & 165.6048 & 329.2183 & 165.1128 & $\mathbf{3}$ \\
\hline $\mathbf{1 0}$ & 1141.5888 & 571.2980 & 1123.5782 & 562.2927 & S & 234.1448 & 117.5761 & 217.1183 & 109.0628 & 216.1343 & 108.5708 & $\mathbf{2}$ \\
\hline $\mathbf{1 1}$ & & & & & K & 147.1128 & 74.0600 & 130.0863 & 65.5468 & & & $\mathbf{1}$ \\
\hline
\end{tabular}
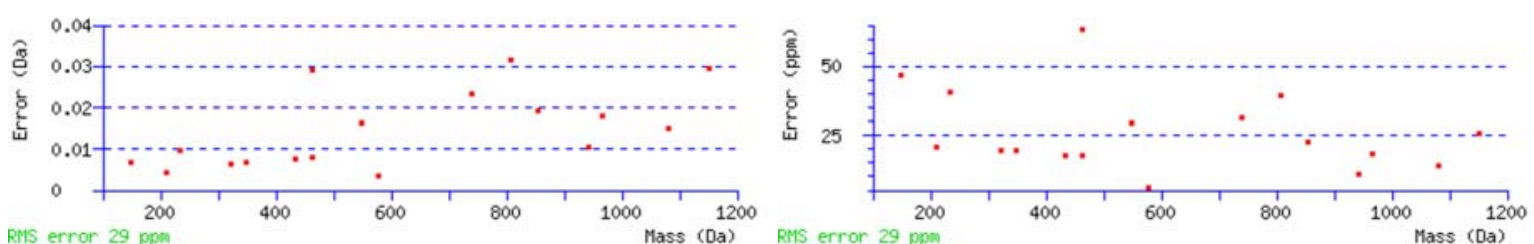

RMS error 29 ppm

PMS error 29 ppe 


\section{Peptide View}

MS/MS Fragmentation of HLIPAANTGESK

Found in gi|31981925|ref|NP_033562.2|, tyrosine 3-monooxygenase/tryptophan 5-monooxygenase activation protein, epsilon polypeptide [Mus mu

Match to Query 264: 1236.699248 from(619.356900,2+)

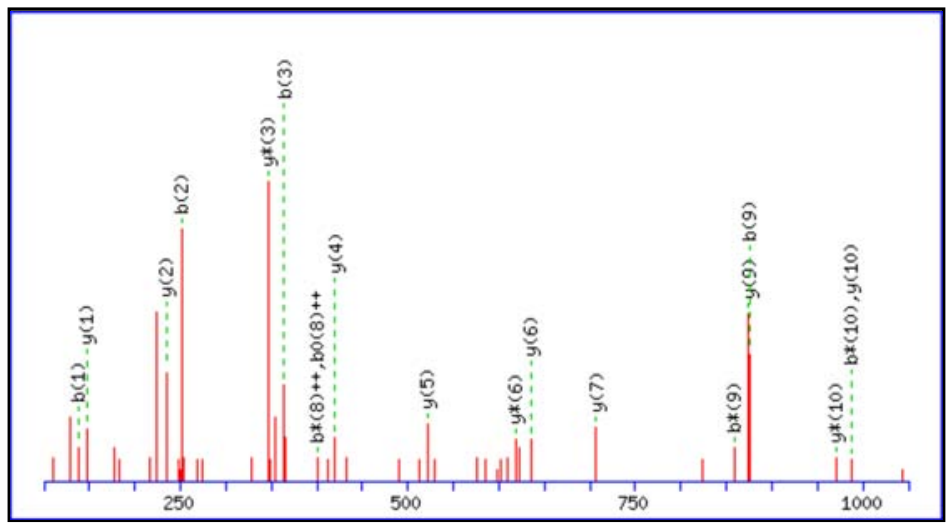

Monoisotopic mass of neutral peptide $\operatorname{Mr}(\mathrm{calc}): 1236.6462$

Ions Score: 62 Expect: 0.00089

Matches (Bold Red): 19/104 fragment ions using 27 most intense peaks

\begin{tabular}{|c|c|c|c|c|c|c|c|c|c|c|c|c|c|c|}
\hline$\#$ & b & $\mathrm{b}^{++}$ & $b *$ & $\mathrm{~b} *^{++}$ & $b^{0}$ & $b^{0++}$ & Seq. & $y$ & $y^{++}$ & y* & $y *^{++}$ & $y^{0}$ & $y^{0++}$ & $\#$ \\
\hline 1 & 138.0662 & 69.5367 & & & & & $\mathrm{H}$ & & & & & & & 12 \\
\hline 2 & 251.1502 & 126.0788 & & & & & $\mathrm{~L}$ & 1100.5946 & 550.8009 & 1083.5680 & 542.2877 & 1082.5840 & 541.7957 & 11 \\
\hline 3 & 364.2343 & 182.6208 & & & & & I & 987.5105 & 494.2589 & 970.4840 & 485.7456 & 969.5000 & 485.2536 & 10 \\
\hline 4 & 461.2871 & 231.1472 & & & & & $P$ & 874.4265 & 437.7169 & 857.3999 & 429.2036 & 856.4159 & 428.7116 & 9 \\
\hline 5 & 532.3242 & 266.6657 & & & & & A & 777.3737 & 389.1905 & 760.3472 & 380.6772 & 759.3631 & 380.1852 & 8 \\
\hline 6 & 603.3613 & 302.1843 & & & & & A & 706.3366 & 353.6719 & 689.3101 & 345.1587 & 688.3260 & 344.6667 & 7 \\
\hline 7 & 717.4042 & 359.2057 & 700.3777 & 350.6925 & & & $\mathrm{~N}$ & 635.2995 & 318.1534 & 618.2729 & 309.6401 & 617.2889 & 309.1481 & 6 \\
\hline 8 & 818.4519 & 409.7296 & 801.4253 & 401.2163 & 800.4413 & 400.7243 & $\mathrm{~T}$ & 521.2566 & 261.1319 & 504.2300 & 252.6186 & 503.2460 & 252.1266 & 5 \\
\hline 9 & 875.4734 & 438.2403 & 858.4468 & 429.7270 & 857.4628 & 429.2350 & G & 420.2089 & 210.6081 & 403.1823 & 202.0948 & 402.1983 & 201.6028 & 4 \\
\hline 10 & 1004.5159 & 502.7616 & 987.4894 & 494.2483 & 986.5054 & 493.7563 & $E$ & 363.1874 & 182.0973 & 346.1609 & 173.5841 & 345.1769 & 173.0921 & 3 \\
\hline 11 & 1091.5480 & 546.2776 & 1074.5214 & 537.7644 & 1073.5374 & 537.2723 & $S$ & 234.1448 & 117.5761 & 217.1183 & 109.0628 & 216.1343 & 108.5708 & 2 \\
\hline 12 & & & & & & & $\mathrm{~K}$ & 147.1128 & 74.0600 & 130.0863 & 65.5468 & & & 1 \\
\hline
\end{tabular}
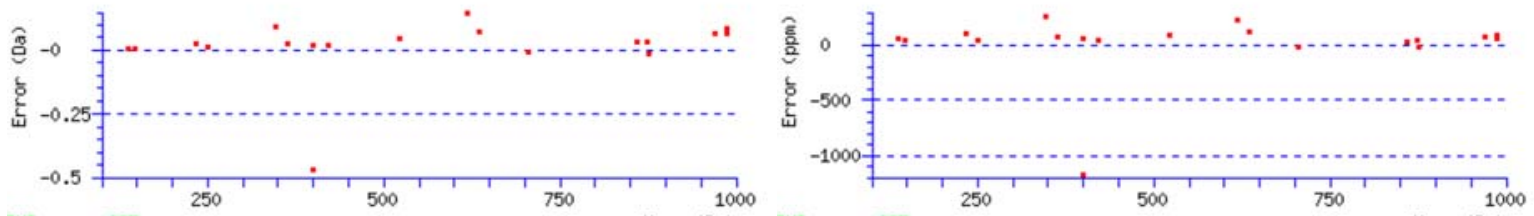

RMS error 287 ppe

Mass (Da) PMS error $287 \mathrm{ppm}$

Mass (Da) 


\section{Peptide View}

MS/MS Fragmentation of HPTQLANSGLK

Found in gi|6753856|ref|NP_034336.1|, fibroblast growth factor receptor 1 [Mus musculus]

Match to Query 220: 1164.638848 from $(583.326700,2+)$

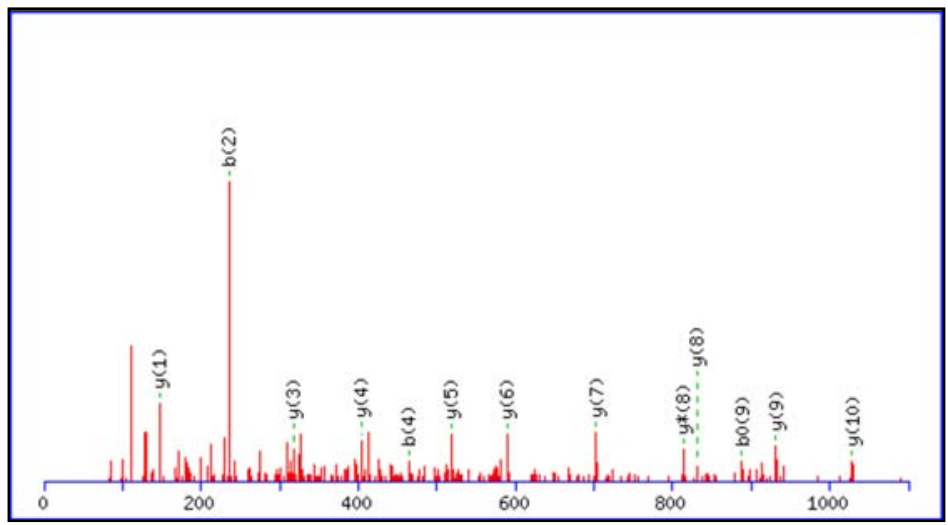

Monoisotopic mass of neutral peptide $\operatorname{Mr}$ (calc): 1164.6251

Ions Score: 48 Expect: 0.021

Matches (Bold Red): 13/104 fragment ions using 32 most intense peaks

\begin{tabular}{|c|c|c|c|c|c|c|c|c|c|c|c|c|c|c|}
\hline$\#$ & b & $\mathrm{b}^{++}$ & $b *$ & $b *^{++}$ & $b^{0}$ & $b^{0++}$ & Seq. & y & $y^{++}$ & y* & $y *^{++}$ & $y^{0}$ & $y^{0++}$ & $\#$ \\
\hline 1 & 138.0662 & 69.5367 & & & & & $\mathrm{H}$ & & & & & & & 11 \\
\hline 2 & 235.1189 & 118.0631 & & & & & $P$ & 1028.5735 & 514.7904 & 1011.5469 & 506.2771 & 1010.5629 & 505.7851 & 10 \\
\hline 3 & 336.1666 & 168.5870 & & & 318.1561 & 159.5817 & $\mathrm{~T}$ & 931.5207 & 466.2640 & 914.4942 & 457.7507 & 913.5101 & 457.2587 & 9 \\
\hline 4 & 464.2252 & 232.6162 & 447.1987 & 224.1030 & 446.2146 & 223.6110 & $Q$ & 830.4730 & 415.7402 & 813.4465 & 407.2269 & 812.4625 & 406.7349 & 8 \\
\hline 5 & 577.3093 & 289.1583 & 560.2827 & 280.6450 & 559.2987 & 280.1530 & L & 702.4145 & 351.7109 & 685.3879 & 343.1976 & 684.4039 & 342.7056 & 7 \\
\hline 6 & 648.3464 & 324.6768 & 631.3198 & 316.1636 & 630.3358 & 315.6715 & A & 589.3304 & 295.1688 & 572.3038 & 286.6556 & 571.3198 & 286.1636 & 6 \\
\hline 7 & 762.3893 & 381.6983 & 745.3628 & 373.1850 & 744.3787 & 372.6930 & $\mathrm{~N}$ & 518.2933 & 259.6503 & 501.2667 & 251.1370 & 500.2827 & 250.6450 & 5 \\
\hline 8 & 849.4213 & 425.2143 & 832.3948 & 416.7010 & 831.4108 & 416.2090 & S & 404.2504 & 202.6288 & 387.2238 & 194.1155 & 386.2398 & 193.6235 & 4 \\
\hline 9 & 906.4428 & 453.7250 & 889.4162 & 445.2118 & 888.4322 & 444.7198 & $G$ & 317.2183 & 159.1128 & 300.1918 & 150.5995 & & & 3 \\
\hline 10 & 1019.5269 & 510.2671 & 1002.5003 & 501.7538 & 1001.5163 & 501.2618 & L & 260.1969 & 130.6021 & 243.1703 & 122.0888 & & & 2 \\
\hline 11 & & & & & & & $\mathrm{~K}$ & 147.1128 & 74.0600 & 130.0863 & 65.5468 & & & 1 \\
\hline
\end{tabular}
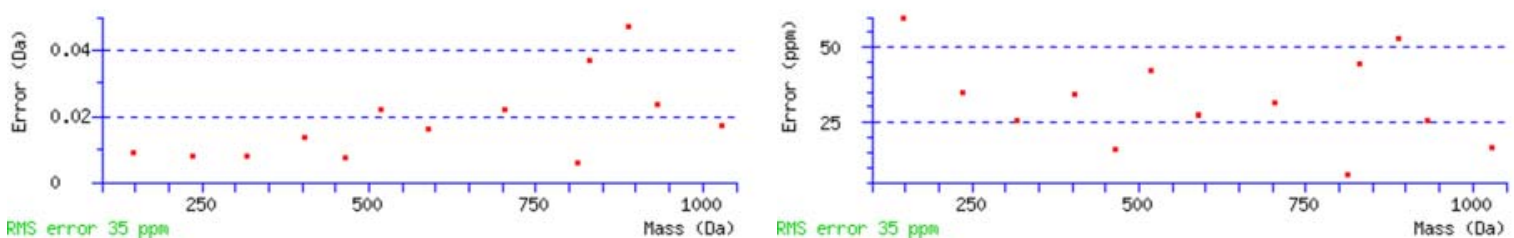

RMS error 35 ppin 


\section{Peptide View}

MS/MS Fragmentation of IKEEYEVAEMGAPHGSASVR

Found in gi|21539593|ref|NP_079654.1|, interferon induced transmembrane protein 3 [Mus musculus]

Match to Query 687: 2169.104472 from(724.042100,3+)

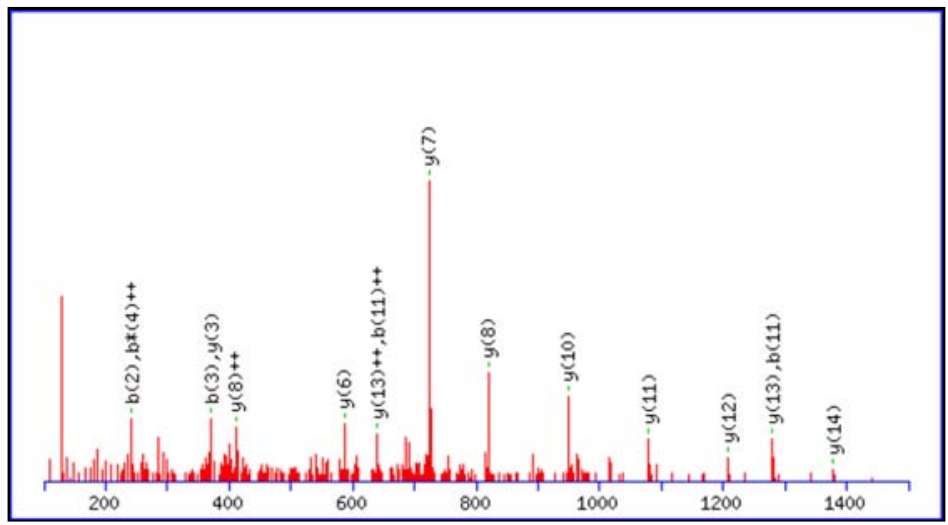

Monoisotopic mass of neutral peptide $\operatorname{Mr}(\mathrm{calc}): 2169.0652$

Variable modifications:

R20 : Arginine-13C615N4 (R-full)

Ions Score: 75 Expect: 0.00019

Matches (Bold Red): 16/218 fragment ions using 17 most intense peaks

\begin{tabular}{|c|c|c|c|c|c|c|c|c|c|c|c|c|c|c|}
\hline \# & b & $b^{++}$ & b* & $b *^{++}$ & $b^{0}$ & $b^{0++}$ & Seq. & $\mathbf{y}$ & ++ & $\mathrm{y} *$ & $y *^{++}$ & $y^{0}$ & $y^{0++}$ & \# \\
\hline 1 & 114.0913 & 5493 & & & & & . & & & & & & & 20 \\
\hline 2 & 42.1863 & 21.5968 & 97 & 1 & & & k & 84 & & & & 779 & & 619 \\
\hline 3 & 1.2289 & 6.1181 & 3 & 348 & 353.2 & 28 & E & 935 & 04 & 669 & .4371 & 1910.8829 & 451 & 18 \\
\hline 4 & 500.2715 & 250.6394 & 483.2449 & 242.1261 & 482.2609 & 241.6341 & L & 1799.8509 & 900. & 1782 & 9158 & 1781.8403 & 38 & 17 \\
\hline 5 & 663.3348 & 32.1710 & 646.3083 & 6578 & 645.3242 & 323.1658 & $Y$ & 1670.8083 & 835.9078 & 7817 & 3945 & 1652.7977 & 025 & 516 \\
\hline 6 & 792.3774 & 396.6923 & 775.3508 & 388.1791 & 774.3668 & 387.6871 & ᄃ & 1507.7450 & 754.3761 & 1490.7184 & 745.8628 & 1489.7344 & 745.3708 & 15 \\
\hline 7 & 891.4458 & 446.2265 & 874.4193 & 437.7133 & 873.4352 & 437.2213 & V & 1378.7024 & 689.8548 & 1361.6758 & 681.3416 & 1360.6918 & 680.8495 & 514 \\
\hline 8 & 962.4829 & 481.7451 & 945.4564 & 473.2318 & 944.4724 & 398 & 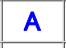 & 1279.6340 & 640.3206 & 126 & 073 & 34 & & 13 \\
\hline 9 & 1091.5255 & 546.2664 & 1074.4990 & 537.7531 & 1073.5149 & 537.2611 & $E$ & 1208.5969 & 604.8021 & 1191.5703 & 596.2888 & 1190.5863 & 595.7968 & 12 \\
\hline 0 & 1222.5660 & 611.7866 & 1205.5394 & 603.2734 & 1204 & & $M$ & 107 & 540.2808 & 106 & 531.7675 & 1061.5437 & & 51 \\
\hline 11 & 1279.5874 & 640.2974 & 1262.5609 & 631.7841 & 1261.5769 & 631.2921 & G & 948.5138 & 474.7605 & 931.4872 & 466.2473 & 930.5032 & 465.7552 & 210 \\
\hline 2 & 1350.6246 & 75.8159 & 1333.5980 & 667.3026 & 1332.6140 & 666. & 1 & 891.4923 & 446.2498 & 874.4658 & 437.7365 & 873.4818 & 437.2445 & 0 \\
\hline 13 & 1447.6773 & 724.3423 & 1430.6508 & 715.8290 & 1429.6668 & 715.3370 & $P$ & 820.4552 & 410.7312 & 803.4287 & 402.2180 & 802.4447 & 401.7260 & 8 \\
\hline 4 & 1584.7362 & 2.8718 & 1567.7097 & 784.3585 & 1566.7257 & 783.8665 & $\mathrm{H}$ & 723.4025 & 362.2049 & 706.3759 & 353.6916 & 705.3919 & 353.1996 & 7 \\
\hline 15 & 1641.7577 & 821.3825 & \begin{tabular}{|l|}
1624.7311 \\
\end{tabular} & 812.8692 & 1623.7471 & 812.3772 & G & 586.3435 & 293.6754 & 569.3170 & 285.1621 & 568.3330 & 284.6701 & 6 \\
\hline 16 & 897 & 864.8985 & 1711 & 856 & 1710 & 855 & 5 & 221 & 265.1647 & 512.2955 & 256.6514 & 511.3115 & 256.1594 & 4 \\
\hline 17 & 1799.8268 & 900.4171 & 1782.8003 & 891.9038 & 1781.8163 & 891.4118 & A & 442.2901 & 221.6487 & 425.2635 & 213.1354 & 424.2795 & 212.6434 & 4 \\
\hline 18 & 1886.8589 & 943.9331 & 1869 & & 1868.8483 & 934.9278 & $\mathrm{~s}$ & & 186.1301 & 354.2264 & 177.6168 & 353.2424 & 177.1248 & 3 \\
\hline 19 & 1985.9273 & 993.4673 & 1968.9007 & 984.9540 & 1967.9167 & 984.4620 & V & 284.2209 & 142.6141 & 267.1944 & 134.1008 & & & 2 \\
\hline 20 & & & & & & & $\mathrm{R}$ & 185.1525 & 93.0799 & 168.1260 & 84.5666 & & & \\
\hline
\end{tabular}
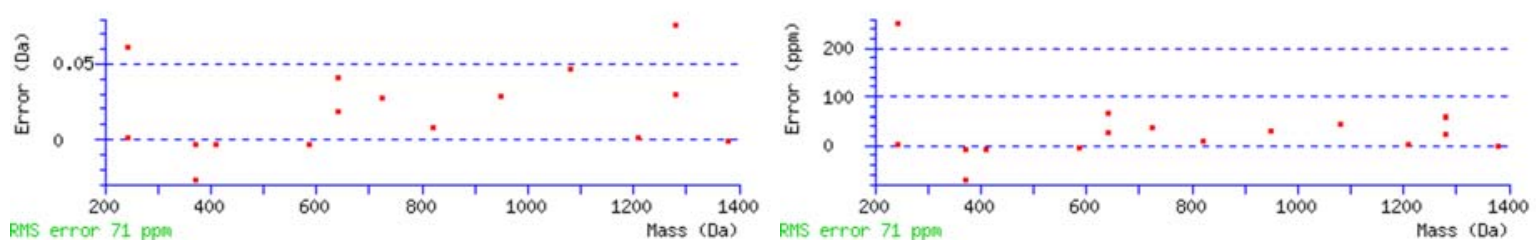


\section{Peptide View}

MS/MS Fragmentation of ILADLEDYLNELWEDKEGK

Found in gi|22203755|ref|NP_666312.1|, eukaryotic translation initiation factor 3, subunit 8 [Mus musculus]

Match to Query 1195: 2292.253272 from(765.091700,3+)

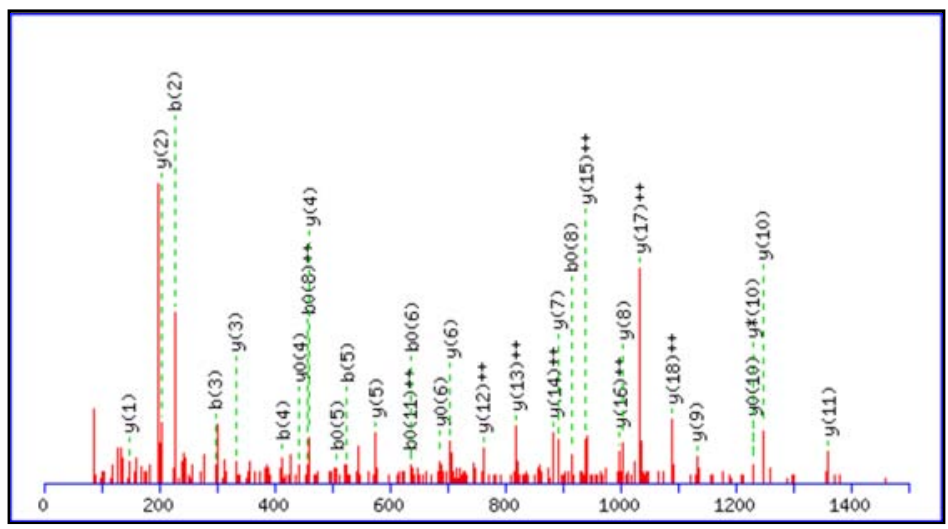

Monoisotopic mass of neutral peptide $\operatorname{Mr}(\mathrm{calc}): 2292.1161$

Ions Score: 51 Expect: 0.049

Matches (Bold Red): 31/188 fragment ions using 94 most intense peaks

\begin{tabular}{|c|c|c|c|c|c|c|c|c|c|c|c|c|c|c|}
\hline \# & b & $b^{++}$ & b* & $\mathrm{b} *^{++}$ & $b^{0}$ & $b^{0++}$ & Seq. & $y$ & $y^{++}$ & y* & $y *^{++}$ & $y^{0}$ & $y^{0++}$ & \# \\
\hline 1 & 114.0913 & 57.5493 & & & & & I & & & & & & & 19 \\
\hline 2 & 227.1754 & 114.0913 & & & & & $\mathrm{~L}$ & 2180.0393 & 1090.5233 & 2163.0128 & 1082.0100 & 2162.0288 & 1081.5180 & 18 \\
\hline 3 & 298.2125 & 149.6099 & & & & & A & 2066.9553 & 1033.9813 & 2049.9287 & 1025.4680 & 2048.9447 & 1024.9760 & 17 \\
\hline 4 & 413.2394 & 207.1234 & & & 395.2289 & 198.1181 & D & 1995.9182 & 998.4627 & 1978.8916 & 989.9494 & 1977.9076 & 989.4574 & 16 \\
\hline 5 & 526.3235 & 263.6654 & & & 508.3129 & 254.6601 & L & 1880.8912 & 940.9492 & 1863.8647 & 932.4360 & 1862.8806 & 931.9440 & 15 \\
\hline 6 & 655.3661 & 328.1867 & & & 637.3555 & 319.1814 & $E$ & 1767.8072 & 884.4072 & 1750.7806 & 875.8939 & 1749.7966 & 875.4019 & 14 \\
\hline 7 & 770.3930 & 385.7002 & & & 752.3825 & 376.6949 & D & 1638.7646 & 819.8859 & 1621.7380 & 811.3726 & 1620.7540 & 810.8806 & 13 \\
\hline 8 & 933.4564 & 467.2318 & & & 915.4458 & 458.2265 & $Y$ & 1523.7376 & 762.3724 & 1506.7111 & 753.8592 & 1505.7271 & 753.3672 & 12 \\
\hline 9 & 1046.5404 & 523.7739 & & & 1028.5299 & 514.7686 & L & 1360.6743 & 680.8408 & 1343.6477 & 672.3275 & 1342.6637 & 671.8355 & 11 \\
\hline 10 & 1160.5834 & 580.7953 & 1143.5568 & 572.2820 & 1142.5728 & 571.7900 & $\mathrm{~N}$ & 1247.5902 & 624.2988 & 1230.5637 & 615.7855 & 1229.5797 & 615.2935 & 10 \\
\hline 11 & 1289.6259 & 645.3166 & 1272.5994 & 636.8033 & 1271.6154 & 636.3113 & $E$ & 1133.5473 & 567.2773 & 1116.5208 & 558.7640 & 1115.5367 & 558.2720 & 9 \\
\hline 12 & 1402.7100 & 701.8586 & 1385.6835 & 693.3454 & 1384.6994 & 692.8534 & L & 1004.5047 & 502.7560 & 987.4782 & 494.2427 & 986.4941 & 493.7507 & 8 \\
\hline 13 & 1588.7893 & 794.8983 & 1571.7628 & 786.3850 & 1570.7788 & 785.8930 & W & 891.4207 & 446.2140 & 874.3941 & 437.7007 & 873.4101 & 437.2087 & 7 \\
\hline 14 & 1717.8319 & 859.4196 & 1700.8054 & 850.9063 & 1699.8213 & 850.4143 & $E$ & 705.3413 & 353.1743 & 688.3148 & 344.6610 & 687.3308 & 344.1690 & 6 \\
\hline 15 & 1832.8588 & 916.9331 & 1815.8323 & 908.4198 & 1814.8483 & 907.9278 & D & 576.2988 & 288.6530 & 559.2722 & 280.1397 & 558.2882 & 279.6477 & 5 \\
\hline 16 & 1960.9538 & 980.9805 & 1943.9273 & 972.4673 & 1942.9432 & 971.9753 & $\mathrm{~K}$ & 461.2718 & 231.1395 & 444.2453 & 222.6263 & 443.2612 & 222.1343 & 4 \\
\hline 17 & 2089.9964 & 1045.5018 & 2072.9698 & 1036.9886 & 2071.9858 & 1036.4966 & $E$ & 333.1769 & 167.0921 & 316.1503 & 158.5788 & 315.1663 & 158.0868 & 3 \\
\hline 18 & 2147.0179 & 1074.0126 & 2129.9913 & 1065.4993 & 2129.0073 & 1065.0073 & $G$ & 204.1343 & 102.5708 & 187.1077 & 94.0575 & & & 2 \\
\hline 19 & & & & & & & $\mathrm{~K}$ & 147.1128 & 74.0600 & 130.0863 & 65.5468 & & & 1 \\
\hline
\end{tabular}
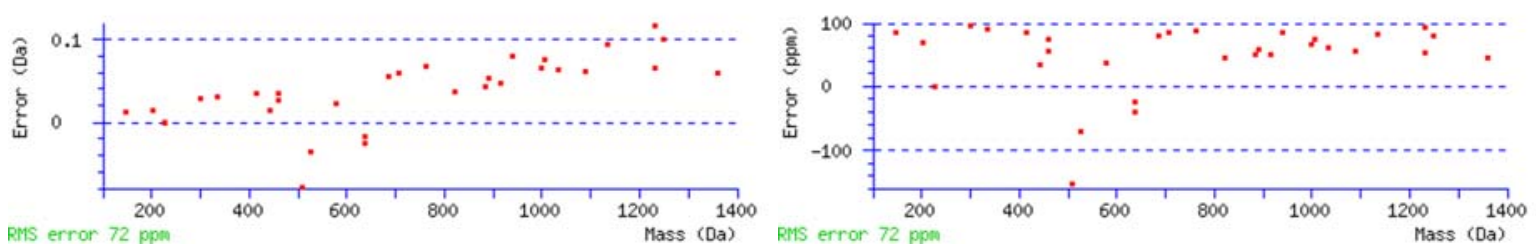


\section{Peptide View}

MS/MS Fragmentation of ILTFDQLALESPK

Found in gi|83699424|ref|NP_033103.2|, ribosomal protein L18 [Mus musculus]

Match to Query 308: 1473.879648 from $(737.947100,2+)$

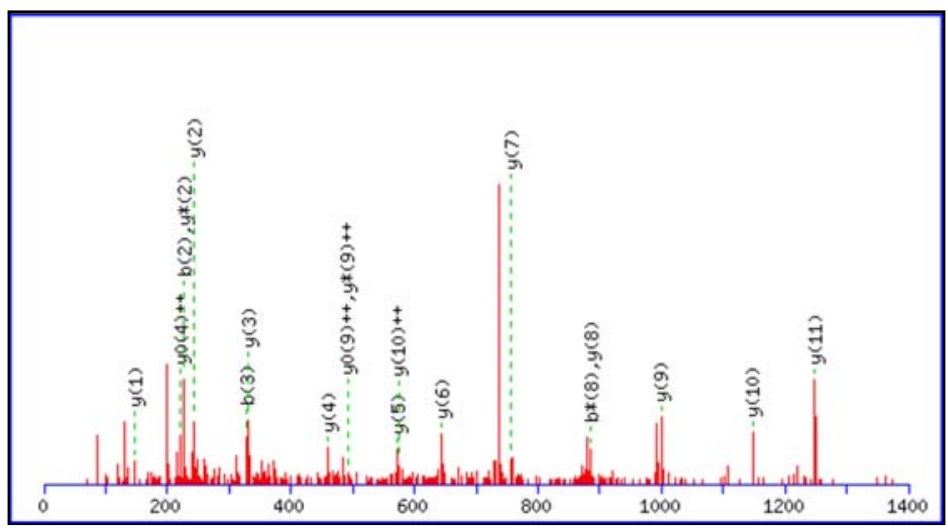

Monoisotopic mass of neutral peptide $\operatorname{Mr}$ (calc): 1473.8079

Ions Score: 46 Expect: 0.048

Matches (Bold Red): 19/126 fragment ions using 63 most intense peaks

\begin{tabular}{|c|c|c|c|c|c|c|c|c|c|c|c|c|c|c|}
\hline \# & b & $\mathrm{b}^{++}$ & $b *$ & $\mathrm{~b} *^{++}$ & $b^{0}$ & $b^{0++}$ & Seq. & $y$ & $\mathrm{y}^{++}$ & y* & $y *^{++}$ & $y^{0}$ & $y^{0++}$ & \# \\
\hline 1 & 114.0913 & 57.5493 & & & & & I & & & & & & & 13 \\
\hline 2 & 227.1754 & 114.0913 & & & & & L & 1361.7311 & 681.3692 & 1344.7045 & 672.8559 & 1343.7205 & 672.3639 & 12 \\
\hline 3 & 328.2231 & 164.6152 & & & 25 & 99 & $T$ & 470 & 62 & & 3139 & 1230.6365 & 615.8219 & 11 \\
\hline 4 & 475.2915 & 238.1494 & & & 457.2809 & 229.1441 & $F$ & 1147.5993 & 574.3033 & 1130.5728 & 565.7900 & 1129.5888 & 565.2980 & 10 \\
\hline 5 & 590.3184 & 295.6629 & & & 572.3079 & 286.6576 & D & 1000.5309 & 500.7691 & 983.5044 & 492.2558 & 982.5204 & 491.7638 & 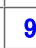 \\
\hline 6 & 718.3770 & 359.6921 & 701.3505 & 351.1789 & 700.3664 & 350.6869 & $Q$ & 885.5040 & 443.2556 & 868.4774 & 434.7424 & 867.4934 & 434.2504 & 8 \\
\hline 7 & 831.4611 & 416.2342 & 814.4345 & 407.7209 & 813.4505 & 407.2289 & $\mathrm{~L}$ & 757.4454 & 379.2263 & 740.4189 & 370.7131 & 739.4348 & 370.2211 & 7 \\
\hline 8 & 902.4982 & 451.7527 & 885.4716 & 443.2395 & 884.4876 & 442.7474 & A & 644.3614 & 322.6843 & 627.3348 & 314.1710 & 626.3508 & 313.6790 & 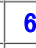 \\
\hline 9 & 1015.5822 & 508.2948 & 998.5557 & 499.7815 & 997.5717 & 499.2895 & L & 573.3242 & 287.1658 & 556.2977 & 278.6525 & 555.3137 & 278.1605 & 5 \\
\hline 10 & 1144.6248 & 572.8161 & 1127.5983 & 564.3028 & 1126.6143 & 563.8108 & E & 460.2402 & 230.6237 & 443.2136 & 222.1105 & 442.2296 & 221.6184 & 4 \\
\hline 11 & 1231.6569 & 616.3321 & 1214.6303 & 607.8188 & 1213.6463 & 607.3268 & $S$ & 331.1976 & 166.1024 & 314.1710 & 157.5892 & 313.1870 & \begin{tabular}{|l|}
157.0972 \\
\end{tabular} & 3 \\
\hline 12 & 1328.7096 & 664.8584 & 1311.6831 & 656.3452 & 1310.6991 & 655.8532 & $\mathrm{P}$ & 244.1656 & 122.5864 & 227.1390 & 114.0731 & & & 2 \\
\hline 13 & & & & & & & $\mathrm{~K}$ & 147.1128 & 74.0600 & 130.0863 & 65.5468 & & & 1 \\
\hline
\end{tabular}
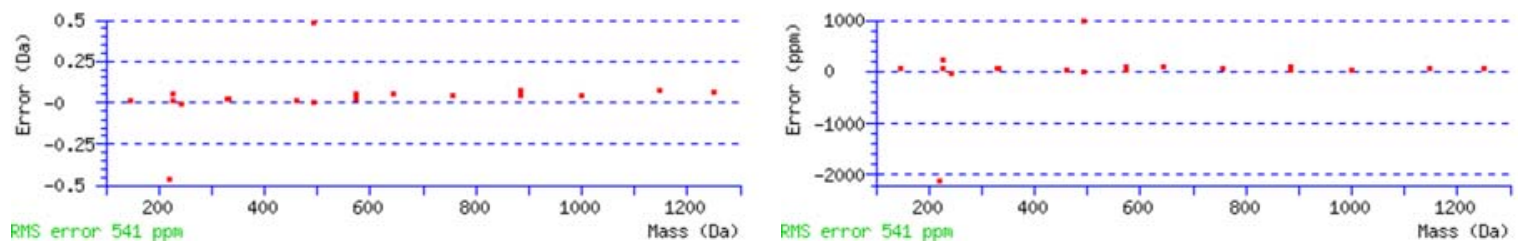


\section{Peptide View}

MS/MS Fragmentation of IQDALSTVLQYAEDVLSGK

Found in gi|21313620|ref|NP_079620.1|, eukaryotic translation initiation factor 3, subunit 5 (epsilon) [Mus musculus]

Match to Query 1120: 2049.219648 from(1025.617100,2+)

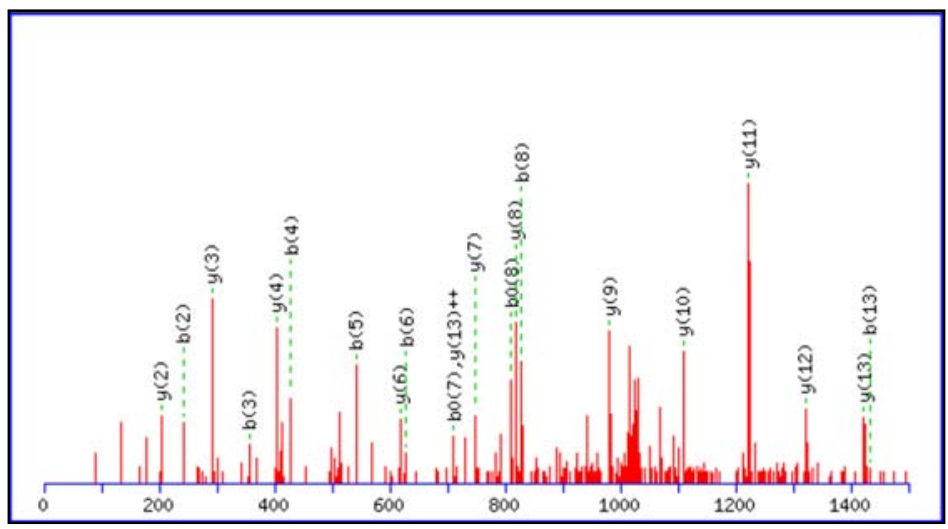

Monoisotopic mass of neutral peptide $\operatorname{Mr}(\mathrm{calc}): 2049.0629$

Ions Score: 81 Expect: $4 \mathrm{e}-005$

Matches (Bold Red): 21/206 fragment ions using 41 most intense peaks

\begin{tabular}{|c|c|c|c|c|c|c|c|c|c|c|c|c|c|c|}
\hline \# & b & $b^{++}$ & b* & $b *^{++}$ & $b^{0}$ & $b^{0++}$ & Seq. & y & $y^{++}$ & y* & $y *^{++}$ & $y^{0}$ & $y^{0++}$ & $\#$ \\
\hline 1 & 114.0913 & 57.5493 & & & & & I & & & & & & & 19 \\
\hline 2 & 242.1499 & 121.5786 & 225.1234 & 113.0653 & & & $Q$ & 1936.9862 & 968.9967 & 1919.9596 & 960.4835 & 1918.9756 & 959.9914 & 18 \\
\hline 3 & 357.1769 & 179.0921 & 340.1503 & 170.5788 & 339.1663 & 170.0868 & D & 1808.9276 & 904.9674 & 1791.9011 & 896.4542 & 1790.9170 & 895.9622 & 17 \\
\hline 4 & 428.2140 & 214.6106 & 411.1874 & 206.0973 & 410.2034 & 205.6053 & A & 1693.9007 & 847.4540 & 1676.8741 & 838.9407 & 1675.8901 & 838.4487 & 16 \\
\hline 5 & 541.2980 & 271.1527 & 524.2715 & 262.6394 & 523.2875 & 262.1474 & L & 1622.8636 & 811.9354 & 1605.8370 & 803.4221 & 1604.8530 & 802.9301 & 15 \\
\hline 6 & 628.3301 & 314.6687 & 611.3035 & 306.1554 & 610.3195 & 305.6634 & $S$ & 1509.7795 & 755.3934 & 1492.7529 & 746.8801 & 1491.7689 & 746.3881 & 14 \\
\hline 7 & 729.3777 & 365.1925 & 712.3512 & 356.6792 & 711.3672 & 356.1872 & $\mathrm{~T}$ & 1422.7475 & 711.8774 & 1405.7209 & 703.3641 & 1404.7369 & 702.8721 & 13 \\
\hline 8 & 828.4461 & 414.7267 & 811.4196 & 406.2134 & 810.4356 & 405.7214 & V & 1321.6998 & 661.3535 & 1304.6732 & 652.8403 & 1303.6892 & 652.3482 & 12 \\
\hline 9 & 941.5302 & 471.2687 & 924.5037 & 462.7555 & 923.5196 & 462.2635 & L & 1222.6314 & 611.8193 & 1205.6048 & 603.3060 & 1204.6208 & 602.8140 & 11 \\
\hline 10 & 1069.5888 & 535.2980 & 1052.5622 & 526.7848 & 1051.5782 & 526.2927 & $\mathbf{Q}$ & 1109.5473 & 555.2773 & 1092.5208 & 546.7640 & 1091.5367 & 546.2720 & 10 \\
\hline 11 & 1232.6521 & 616.8297 & 1215.6256 & 608.3164 & 1214.6416 & 607.8244 & $\mathrm{Y}$ & 981.4887 & 491.2480 & 964.4622 & 482.7347 & 963.4782 & 482.2427 & 9 \\
\hline 12 & 1303.6892 & 652.3483 & 1286.6627 & 643.8350 & 1285.6787 & 643.3430 & A & 818.4254 & 409.7163 & 801.3989 & 401.2031 & 800.4148 & 400.7111 & 8 \\
\hline 13 & 1432.7318 & 716.8695 & 1415.7053 & 708.3563 & 1414.7213 & 707.8643 & $E$ & 747.3883 & 374.1978 & 730.3617 & 365.6845 & 729.3777 & 365.1925 & 7 \\
\hline 14 & 1547.7588 & 774.3830 & 1530.7322 & 765.8697 & 1529.7482 & 765.3777 & D & 618.3457 & 309.6765 & 601.3192 & 301.1632 & 600.3351 & 300.6712 & 6 \\
\hline 15 & 1646.8272 & 823.9172 & 1629.8006 & 815.4039 & 1628.8166 & 814.9119 & V & 503.3188 & 252.1630 & 486.2922 & 243.6497 & 485.3082 & 243.1577 & 5 \\
\hline 16 & 1759.9112 & 880.4593 & 1742.8847 & 871.9460 & 1741.9007 & 871.4540 & L & 404.2504 & 202.6288 & 387.2238 & 194.1155 & 386.2398 & 193.6235 & 4 \\
\hline 17 & 1846.9433 & 923.9753 & 1829.9167 & 915.4620 & 1828.9327 & 914.9700 & $\mathrm{~s}$ & 291.1663 & 146.0868 & 274.1397 & 137.5735 & 273.1557 & 137.0815 & 3 \\
\hline 18 & 1903.9647 & 952.4860 & 1886.9382 & 943.9727 & 1885.9542 & 943.4807 & G & 204.1343 & 102.5708 & 187.1077 & 94.0575 & & & 2 \\
\hline 19 & & & & & & & $\mathrm{~K}$ & 147.1128 & 74.0600 & 130.0863 & 65.5468 & & & 1 \\
\hline
\end{tabular}
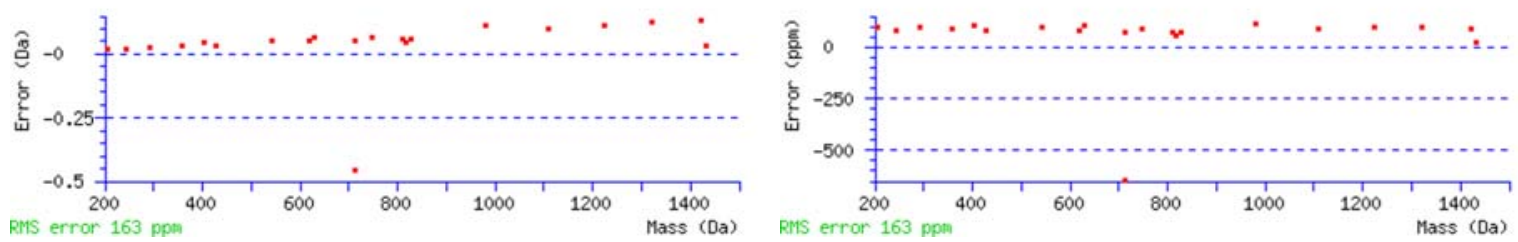


\section{Peptide View}

MS/MS Fragmentation of IQSLVGLSWIDWLK

Found in gi|29789235|ref|NP_083370.1|, phosphatidylinositol 3-kinase, catalytic, beta polypeptide [Mus musculus]

Match to Query 553: 1656.942448 from $\left(829.478500,2^{+}\right)$

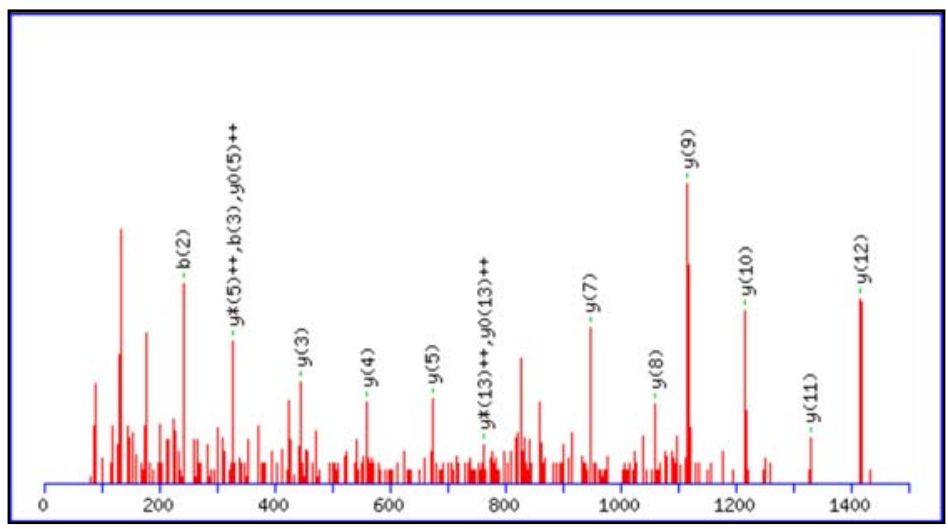

Monoisotopic mass of neutral peptide $\mathrm{Mr}$ (calc): 1656. 9239

Ions Score: 81 Expect: $1.9 \mathrm{e}-005$

Matches (Bold Red): 15/144 fragment ions using 21 most intense peaks

\begin{tabular}{|c|c|c|c|c|c|c|c|c|c|c|c|c|c|c|}
\hline \# & b & $b^{++}$ & $b *$ & $b *^{++}$ & $b^{0}$ & $b^{0++}$ & Seq. & $y$ & + & y* & $y *^{++}$ & $y^{0}$ & $y^{0++}$ & $\#$ \\
\hline 1 & 114.0913 & 57.5493 & & & & & I & & & & & & & 14 \\
\hline 2 & 242.1499 & 121.5786 & 225.1234 & 113.0653 & & & $Q$ & 1544.8471 & 772.9272 & 1527.8206 & 764.4139 & 1526.8365 & 763.9219 & 13 \\
\hline 3 & 329.1819 & 165.0946 & 312.1554 & 156.5813 & 311.1714 & 156 . & $S$ & 1416.7885 & 708.8979 & 1399.7620 & 700.3846 & 1398.7780 & 699.8926 & 12 \\
\hline 4 & 2.2660 & 221.6366 & 95 & 234 & 424 & 14 & L & 1329.7565 & 665.3819 & 1312 & 656.8686 & 459 & & 11 \\
\hline 5 & 541.3344 & 271.1708 & 524.3079 & 262.6576 & 523.3239 & 262.1656 & V & 1216.6724 & 608.8399 & 1199.6459 & 600.3266 & 1198.6619 & 599.8346 & 10 \\
\hline 6 & 598.3559 & 299.6816 & 581.3293 & 291.1683 & 580.3453 & 290.6763 & G & 1117.6040 & 559.3057 & 1100.5775 & 550.7924 & 1099.5935 & 550.3004 & -1 \\
\hline 7 & 711.4399 & 356.2236 & 694.4134 & 347.7103 & 693.4294 & 347.2183 & L & 1060.5826 & 530.7949 & 1043.5560 & 522.2816 & 1042.5720 & 521.7896 & 8 \\
\hline 8 & 798.4720 & 399.7396 & 781.4454 & 391.2263 & 780.4614 & 390.7343 & S & 947.4985 & 474.2529 & 930.4720 & 465.7396 & 929.4879 & 465.2476 & 7 \\
\hline 9 & 984.5513 & 492.7793 & 967.5247 & 484.2660 & 966.5407 & 483.7740 & w & 860.4665 & 430.7369 & 843.4399 & 422.2236 & 842.4559 & 421.7316 & 6 \\
\hline 10 & 1097.6353 & 549.3213 & 1080.6088 & 540.8080 & 1079.6248 & 540.3160 & I & 674.3872 & 337.6972 & 657.3606 & 329.1839 & 656.3766 & 328.6919 & 5 \\
\hline 11 & 1212.6623 & 606.8348 & 1195.6357 & 598.3215 & 1194.6517 & 597.8295 & D & 561.3031 & 281.1552 & 544.2766 & 272.6419 & 543.2925 & 272.1499 & 4 \\
\hline 12 & 1398.7416 & 699.8744 & 1381.7150 & 691.3612 & 1380.7310 & 690.8691 & w & 446.2762 & 223.6417 & 429.2496 & 215.1284 & & & 3 \\
\hline 13 & 1511.8256 & 756.4165 & 1494.7991 & 747.9032 & 1493.8151 & 747.4112 & $\mathrm{~L}$ & 260.1969 & 130.6021 & 243.1703 & 122.0888 & & & 2 \\
\hline 14 & & & & & & & $\mathrm{~K}$ & 147.1128 & 74.0600 & 130.0863 & 65.5468 & & & 1 \\
\hline
\end{tabular}
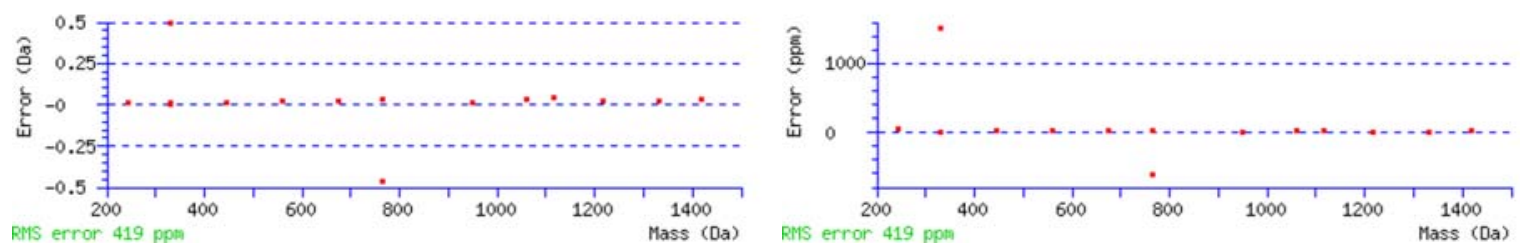


\section{Peptide View}

MS/MS Fragmentation of ISNNNNGLTEVLK

Found in gi|27370424|ref|NP_766512.1|, hypothetical protein LOC244895 [Mus musculus]

Match to Query 270: 1414.802048 from $(708.408300,2+)$

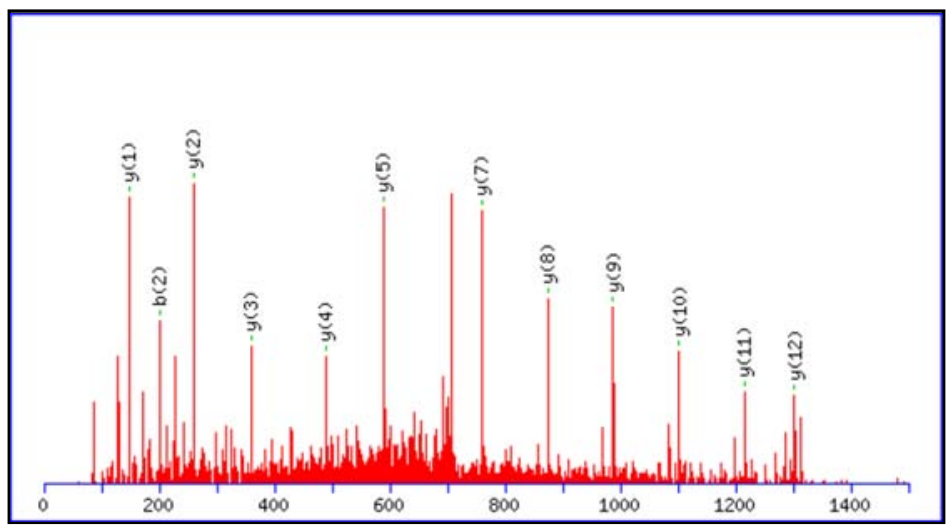

Monoisotopic mass of neutral peptide $\mathrm{Mr}$ (calc): 1414.7415

Ions Score: 110 Expect: 1.8e-008

Matches (Bold Red): 12/132 fragment ions using 24 most intense peaks

\begin{tabular}{|c|c|c|c|c|c|c|c|c|c|c|c|c|c|c|}
\hline \# & b & $b^{++}$ & b* & $b *^{++}$ & $b^{0}$ & $1++$ & Seq. & y & $y^{++}$ & y* & $y *^{++}$ & $y^{0}$ & $y^{0++}$ & \# \\
\hline 1 & 4.0913 & 93 & & & & & I & & & & & & & 1 \\
\hline 2 & 201.1234 & 101.0653 & & & 28 & 0 & S & 02. & 360 & 85.6 & 28 & 128 & ( & t \\
\hline 3 & 1663 & 158.0868 & 77 & 149 & 557 & 149.0815 & $\mathrm{~N}$ & 1215.6328 & 3200 & 1198.6062 & 3068 & 1197.6222 & 3147 & 11 \\
\hline 4 & 092 & 1083 & 7 & 50 & 37 & 206.1030 & $\mathrm{~N}$ & 399 & .2986 & 1084.5633 & 542.7853 & 1083.5793 & 542.2933 & \\
\hline 5 & 543.2522 & 272.1297 & 526.2256 & 263.6164 & 525.2416 & 263.1244 & $\mathrm{~N}$ & 987.5469 & 494.2771 & 970.5204 & 485.7638 & 969.5364 & 485.2718 & \\
\hline 6 & 7.2951 & 329.1512 & 5 & 320 & & 320 & $\mathrm{~N}$ & 40 & 56 & & 424 & 934 & 428 & \\
\hline 7 & 714.3165 & 357.6619 & 697.2900 & 349.1486 & 696. & 348.6566 & $G$ & 759.4611 & 380.2342 & 742.4345 & 371.7209 & 741.4505 & 371.2289 & \\
\hline 8 & 827.4006 & 414.2039 & 810.3741 & 405.6907 & 809.3900 & 405.1987 & L & 2.4396 & 351.7234 & 685.4131 & 343.2102 & 684.4290 & 342.7182 & \\
\hline 9 & 928.4483 & 464.7278 & 911.4 & 45 & 910 & 455.7225 & $\mathrm{~T}$ & 555 & 1814 & 2.3290 & 681 & 50 & 286 & 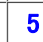 \\
\hline 10 & 1057.4909 & 529.2491 & 1040.4643 & 520.7358 & 1039.4803 & 520.2438 & $E$ & 3079 & 244.6576 & 813 & 236.1443 & 470.2973 & 235.65 & \\
\hline 11 & 1156.5593 & 578.7833 & 1139.5 & 570.2700 & 1138.5487 & 569.7780 & V & 359.2653 & 180.1363 & 342.2387 & 171.6230 & & & \\
\hline 12 & 1269.6433 & 635.3253 & 1252.6168 & 626.8120 & 1251.6328 & 626.3200 & L & 260.1969 & 130.6021 & 243.1 & 122.0888 & & & \\
\hline 3 & & & & & & & $\mathrm{~K}$ & 147.1128 & 74.0600 & 130.0863 & 65.5468 & & & \\
\hline
\end{tabular}
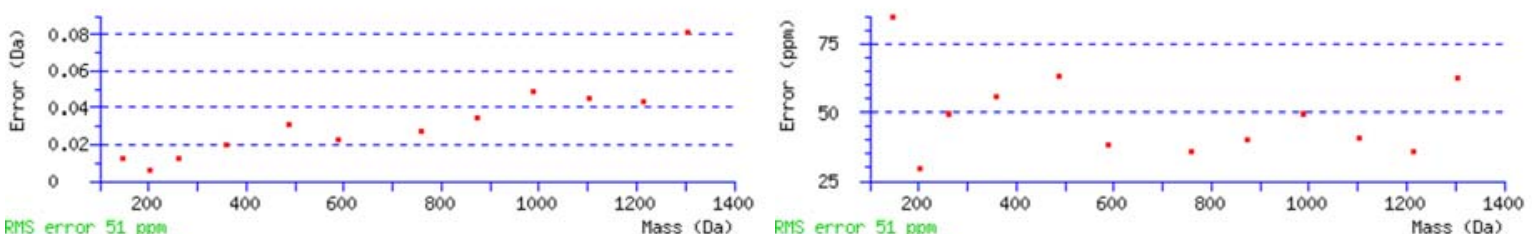


\section{Peptide View}

MS/MS Fragmentation of KLEAAATALATK

Found in gi|30794450|ref|NP_077174.1|, ribosomal protein L4 [Mus musculus]

Match to Query 213: 1186.750848 from $\left(594.382700,2^{+}\right)$

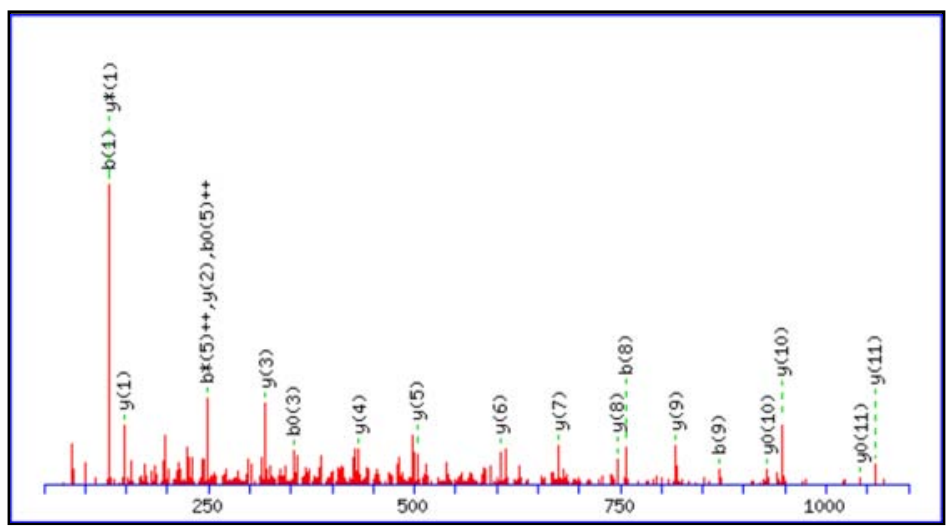

Monoisotopic mass of neutral peptide $\mathrm{Mr}$ (calc): 1186.6921

Ions Score: 92 Expect: $8.8 \mathrm{e}-007$

Matches (Bold Red): 21/126 fragment ions using 34 most intense peaks

\begin{tabular}{|c|c|c|c|c|c|c|c|c|c|c|c|c|c|c|}
\hline$\#$ & b & $b^{++}$ & b* & $b *^{++}$ & $b^{0}$ & $b^{0++}$ & Seq. & $y$ & $y^{++}$ & y* & $y *^{++}$ & $y^{0}$ & $y^{0++}$ & \# \\
\hline 1 & 129.1022 & 65.0548 & 112.0757 & 56.5415 & & & $\mathrm{~K}$ & & & & & & & 12 \\
\hline 2 & 242.1863 & 121.5968 & 225.1597 & 113.0835 & & & L & 1059.6044 & 530.3058 & 1042.5779 & 521.7926 & 1041.5939 & 521.3006 & 11 \\
\hline 3 & 371.2289 & 186.1181 & 354.2023 & 177.6048 & 353.2183 & \begin{tabular}{|l|}
177.1128 \\
\end{tabular} & $E$ & 946.5204 & 473.7638 & 929.4938 & 465.2505 & 928.5098 & 464.7585 & 10 \\
\hline 4 & 442.2660 & 221.6366 & 425.2394 & 213.1234 & 424.2554 & 212.6314 & A & 817.4778 & 409.2425 & 800.4512 & 400.7292 & 799.4672 & 400.2372 & 9 \\
\hline 5 & 513.3031 & 257.1552 & 496.2766 & 248.6419 & 495.2925 & 248.1499 & $A$ & 746.4407 & 373.7240 & 729.4141 & 365.2107 & 728.4301 & 364.7187 & 8 \\
\hline 6 & 584.3402 & 292.6737 & 567.3137 & 284.1605 & 566.3297 & 283.6685 & $A$ & 675.4036 & 338.2054 & 658.3770 & 329.6921 & 657.3930 & 329.2001 & 7 \\
\hline 7 & 685.3879 & 343.1976 & 668.3613 & 334.6843 & 667.3773 & 334.1923 & $T$ & 604.3664 & 302.6869 & 587.3399 & 294.1736 & 586.3559 & 293.6816 & 6 \\
\hline 8 & 756.4250 & 378.7161 & 739.3985 & 370.2029 & 738.4144 & 369.7109 & $A$ & 503.3188 & 252.1630 & 486.2922 & 243.6497 & 485.3082 & 243.1577 & 5 \\
\hline 9 & 869.5091 & 435.2582 & 852.4825 & 426.7449 & 851.4985 & 426.2529 & L & 432.2817 & 216.6445 & 415.2551 & 208.1312 & 414.2711 & 207.6392 & 4 \\
\hline 10 & 940.5462 & 470.7767 & 923.5196 & 462.2635 & 922.5356 & 461.7714 & $A$ & 319.1976 & 160.1024 & 302.1710 & 151.5892 & 301.1870 & 151.0972 & 3 \\
\hline 11 & 1041.5939 & 521.3006 & 1024.5673 & 512.7873 & 1023.5833 & 512.2953 & $T$ & 248.1605 & 124.5839 & 231.1339 & 116.0706 & 230.1499 & 115.5786 & 2 \\
\hline 12 & & & & & & & K & 147.1128 & 74.0600 & 130.0863 & 65.5468 & & & 1 \\
\hline
\end{tabular}
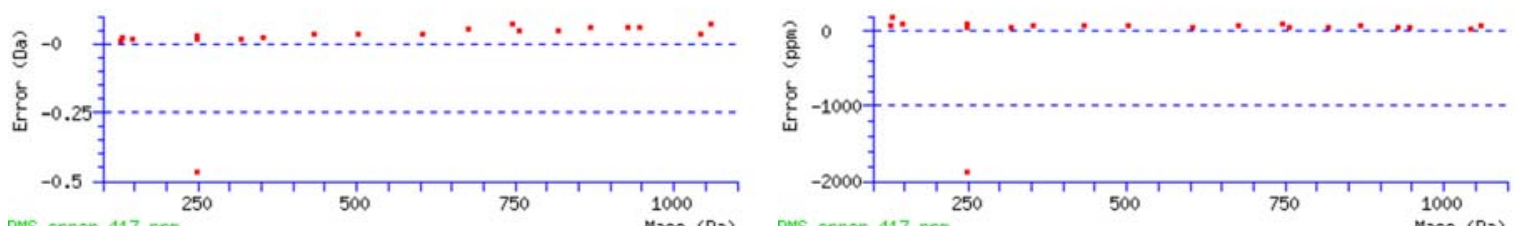

RMS error $417 \mathrm{ppm}$ 


\section{Peptide View}

MS/MS Fragmentation of KLQEEELLATHVDMR

Found in gi|71043975|ref|NP_080478.2|, Ymer protein isoform 1 [Mus musculus]

Match to Query 595: 1810.970772 from(604.664200,3+)

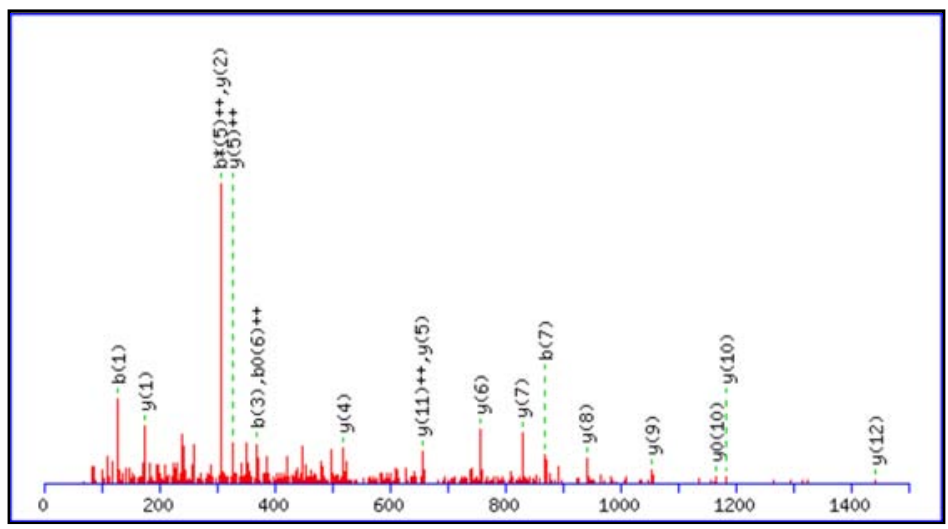

Monoisotopic mass of neutral peptide $\mathrm{Mr}$ (calc): 1810.9247

Ions Score: 84 Expect: 1.1e-005

Matches (Bold Red): 18/158 fragment ions using 22 most intense peaks

\begin{tabular}{|c|c|c|c|c|c|c|c|c|c|c|c|c|c|c|}
\hline \# & b & $b^{++}$ & $b *$ & $b *^{++}$ & $b^{0}$ & $b^{0++}$ & Seq. & $y$ & + & y* & $*^{++}$ & $y^{0}$ & $y^{0++}$ & \# \\
\hline 1 & 129.1022 & 65.0548 & 112.0757 & 56.5415 & & & $\mathrm{~K}$ & & & & & & & 15 \\
\hline 2 & 2.1863 & 121. & 97 & 1 & & & L & & & 166 & & 166 & 169 & 14 \\
\hline 3 & 370.2449 & 185.6261 & 353.2183 & 177.1128 & & & $Q$ & 1570.7530 & 785. & 1553 & 68 & 1552.7424 & & 13 \\
\hline 4 & 75 & 4 & 9 & 41 & 9 & & 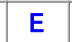 & & & & & & & 12 \\
\hline 5 & 628.3301 & 314.6687 & 611.3035 & 306.1554 & 610.3195 & 305.6634 & $E$ & 1313.6518 & 657.3295 & 1296.6252 & 163 & 1295.6412 & 648 & 11 \\
\hline 6 & 7.3726 & 379.1900 & 740.3461 & 3 & 39.3621 & & $E$ & 1184.6092 & 592 & & 950 & 1166 & & 10 \\
\hline 7 & \begin{tabular}{|l|}
870.4567 \\
\end{tabular} & 435.7320 & 853.4302 & \begin{tabular}{|l|}
427.2187 \\
\end{tabular} & 852.4461 & 426.7267 & L & 1055.5666 & 528.2869 & 103 & 519.7737 & 1037.5560 & 519.2817 & 9 \\
\hline 8 & 983.5408 & 492.2740 & 966.5142 & 483.7607 & 965.5302 & 483.2687 & L & 942.4825 & 471.7449 & 4560 & 463.2316 & 720 & 462.7396 & 8 \\
\hline 9 & 1054.5779 & 527.7926 & 1037.5513 & 519.2793 & 1036.5673 & 518.7873 & A & & 415.2029 & .3719 & 406.6896 & & & 7 \\
\hline 10 & 1155.6256 & 578.3164 & 1138.5990 & 569.8031 & 1137.6150 & 569.3111 & $\mathrm{~T}$ & 758.3614 & 379.6843 & 741.3348 & 371.1710 & 508 & 370.6790 & 6 \\
\hline 11 & 1292.6845 & 646.8459 & 1275.6579 & 638.3326 & 1274.6739 & 637.8406 & $\mathrm{H}$ & 657.3137 & 329.1605 & 640.2871 & 320.6472 & 639.3031 & 320.1552 & 5 \\
\hline 12 & 1391.7529 & 696.3801 & 1374.7263 & \begin{tabular}{|l|}
687.8668 \\
\end{tabular} & 1373.7423 & 687.3748 & $\mathrm{~V}$ & 520.2548 & 260.6310 & 503.2282 & 252.1178 & 02.2442 & 251.6257 & 4 \\
\hline 13 & 1506.7798 & 753.8935 & 1489.7533 & \begin{tabular}{|l|}
745.3803 \\
\end{tabular} & 1488.7693 & 744.8883 & D & 21.1864 & 211.0968 & 404.1598 & 202.5835 & 403.1758 & 202.0915 & 3 \\
\hline 14 & 1637.8203 & 819.4138 & 1620.7937 & 810.9005 & 1619.8097 & 810.4085 & $M$ & 306.1594 & 153.5834 & 289.1329 & 145.0701 & & & 2 \\
\hline 15 & & & & & & & $\mathrm{R}$ & 175.1190 & 88.0631 & 158.0924 & 79.5498 & & & \\
\hline
\end{tabular}
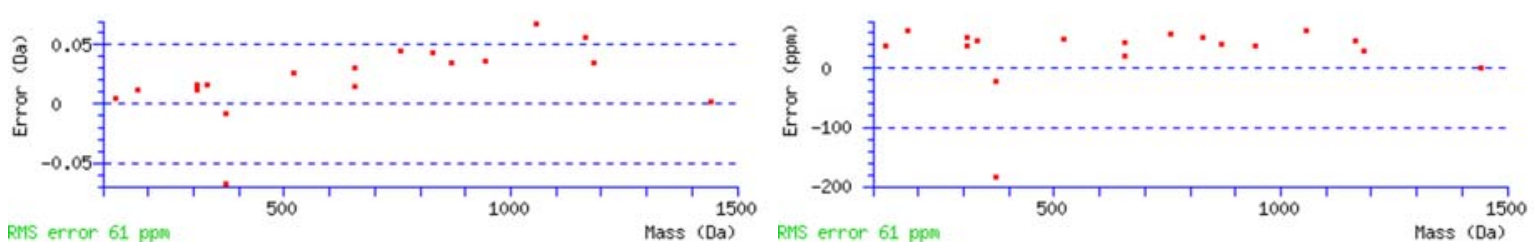


\section{Peptide View}

MS/MS Fragmentation of KLWMSLGDSWVK

Found in gi|75677397|ref|NP_031971.2|, epidermal growth factor receptor pathway substrate 8 [Mus musculus]

Match to Query 445: 1448.758248 from $(725.386400,2+)$

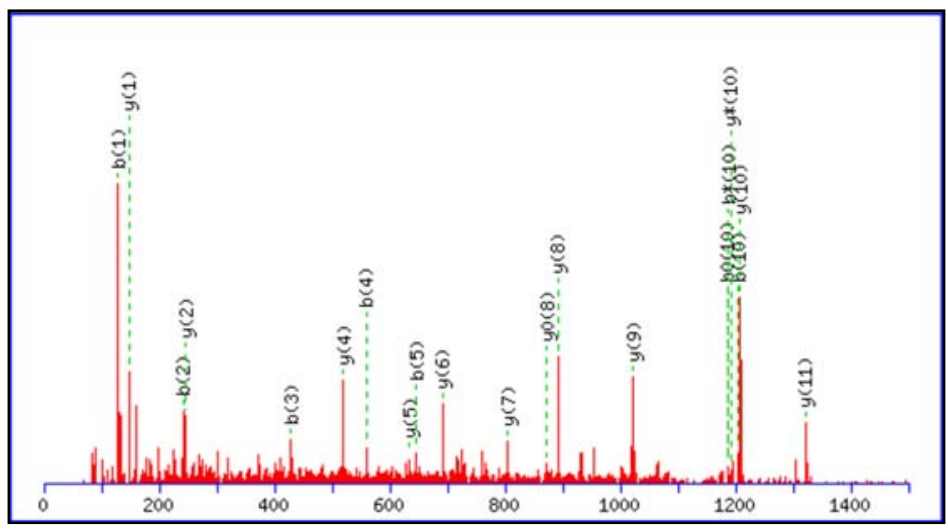

Monoisotopic mass of neutral peptide $\operatorname{Mr}(\mathrm{calc}): 1448.7486$

Ions Score: 66 Expect: 0.00042

Matches (Bold Red) : 20/118 fragment ions using 52 most intense peaks

\begin{tabular}{|c|c|c|c|c|c|c|c|c|c|c|c|c|c|c|}
\hline$\#$ & b & $b^{++}$ & $b *$ & $\mathrm{~b} *^{++}$ & $b^{0}$ & $b^{0++}$ & Seq. & y & $y^{++}$ & y* & $y *^{++}$ & $y^{0}$ & $y^{0++}$ & $\#$ \\
\hline 1 & 129.1022 & 65.0548 & 112.0757 & 56.5415 & & & $\mathrm{~K}$ & & & & & & & 12 \\
\hline 2 & 242.1863 & 121.5968 & 225.1597 & 113.0835 & & & $L$ & 1321.6609 & 661. & 1304.6343 & 652.8208 & 1303.6503 & 652.3288 & 11 \\
\hline 3 & 428.2656 & 214.6364 & 411.2391 & 206.1232 & & & $w$ & 1208.5768 & 604.7921 & 1191.5503 & 596.2788 & 1190.5663 & 595.7868 & 10 \\
\hline 4 & 559.3061 & 280.1567 & 542.2795 & 271.6434 & & & $M$ & 22.4975 & 511.7524 & 1005.4710 & 503.2391 & 1004.4870 & 502.7471 & 9 \\
\hline 5 & 646.3381 & 323.6727 & 629.3116 & 315.1594 & 628.3276 & 674 & $\mathrm{~s}$ & 891.4570 & 446.2322 & 874.4305 & 437.7189 & 873.4465 & 437.2269 & 8 \\
\hline 6 & 759.4222 & 380.2147 & 742. & 371.7015 & 16 & 094 & L & 250 & 161 & 985 & 394.2029 & 786.4144 & 393.7109 & 7 \\
\hline 7 & 816.4436 & 408.7255 & 799.4171 & 400.2122 & 798.4331 & 399.7202 & G & 691.3410 & 346.1741 & 1.3144 & 337.6608 & 673.3304 & 337.1688 & 6 \\
\hline 8 & 931.4706 & 466.2389 & 914.4440 & 457.7257 & 913.4600 & 457.2336 & D & 634.3195 & 317.6634 & 617.2929 & 309.1501 & 616.3089 & 308.6581 & 5 \\
\hline 9 & 1018.5026 & 509.7549 & 1001.4761 & 501.2417 & 1000.4920 & 500.7497 & $S$ & 519.2926 & 260.1499 & 2660 & 251.6366 & 501.2820 & 251.1446 & 4 \\
\hline 10 & 1204.5819 & 602.7946 & 1187.5554 & 594.2813 & 1186.5714 & 593.7893 & W & 432.2605 & 216.6339 & 340 & 208.1206 & & & 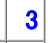 \\
\hline 11 & 1303.6503 & 652.3288 & 1286.6238 & 643.8155 & 1285.6398 & 643.3235 & V & 246.1812 & 123.5942 & 229.1547 & 115.0810 & & & 2 \\
\hline 2 & & & & & & & $\mathrm{~K}$ & 147.1128 & 74.0600 & 130.0863 & 65.5468 & & & \\
\hline
\end{tabular}
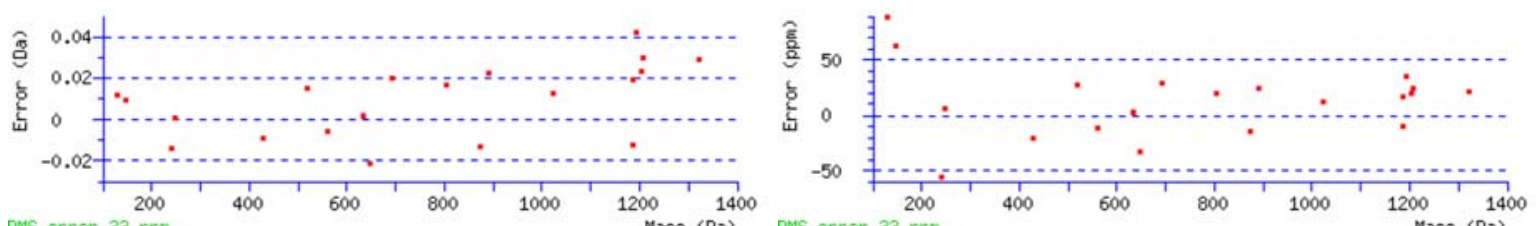

$\begin{array}{ccccccccccccc} & 200 & 400 & 600 & 800 & 1000 & 1200 \\ \text { Mass (Da) }\end{array}$ 


\section{Peptide View}

MS/MS Fragmentation of KPLFGEIGHTIMNLLVDLR

Found in gi|27734996|ref|NP_775568.1|, zinc finger, FYVE domain containing 16 [Mus musculus]

Match to Query 534: 2171.328072 from $(724.783300,3+)$

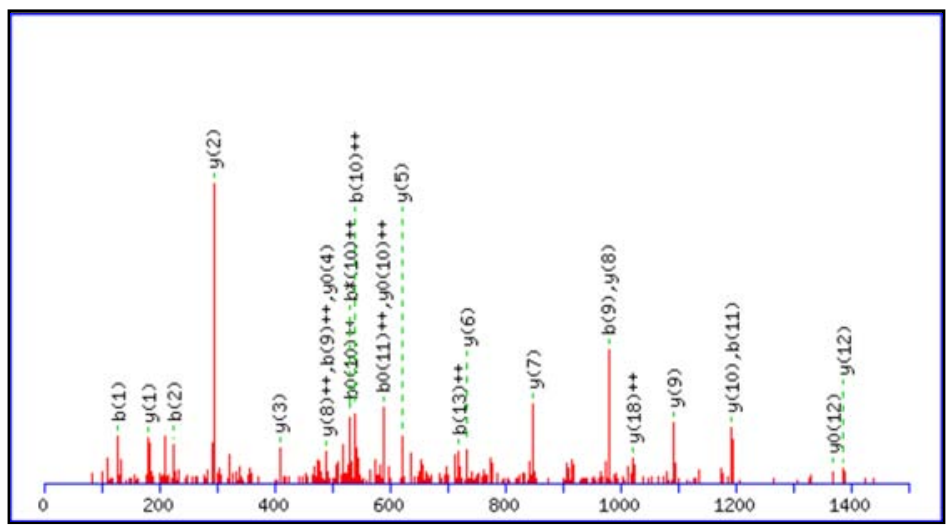

Monoisotopic mass of neutral peptide $\operatorname{Mr}(\mathrm{calc}): 2171.2231$

Variable modifications:

R19 : Arginine-13C6 (R-13C6)

Ions Score: 64 Expect: 0.0021

Matches (Bold Red): 25/202 fragment ions using 37 most intense peaks

\begin{tabular}{|c|c|c|c|c|c|c|c|c|c|c|c|c|c|c|}
\hline \# & b & $\mathrm{b}^{++}$ & $b *$ & $b *^{++}$ & $b^{0}$ & $b^{0++}$ & Seq. & y & $\mathrm{y}^{++}$ & y* & $y *^{++}$ & $y^{0}$ & $y^{0++}$ & $\#$ \\
\hline 1 & 129.1022 & 65.0548 & 112.0757 & 56.5415 & & & K & & & & & & & 19 \\
\hline 2 & 226.1550 & 113.5811 & 209.1284 & 105.0679 & & & $\mathrm{P}$ & 2044.1355 & 1022.5714 & 2027.1089 & 1014.0581 & 2026.1249 & 1013.5661 & 18 \\
\hline 3 & 339.2391 & 170.1232 & 322.2125 & 161.6099 & & & $\mathrm{~L}$ & 1947.0827 & 974.0450 & 1930.0562 & 965.5317 & 1929.0721 & 965.0397 & 77 \\
\hline 4 & 486.3075 & 243.6574 & 469.2809 & 235.1441 & & & $F$ & 1833.9986 & 917.5030 & 1816.9721 & 908.9897 & 1815.9881 & 908.4977 & 76 \\
\hline 5 & 543.3289 & 272.1681 & 526.3024 & 263.6548 & & & $G$ & 1686.9302 & 843.9688 & 1669.9037 & 7835.4555 & 1668.9197 & 834.9635 & 515 \\
\hline 6 & 672.3715 & 336.6894 & 655.3450 & 328.1761 & 654.3610 & 327.6841 & $E$ & 1629.9088 & 815.4580 & 1612.8822 & 806.9448 & 1611.8982 & 806.4527 & 74 \\
\hline 7 & 785.4556 & 393.2314 & 768.4290 & 384.7182 & 767.4450 & 384.2261 & I & 1500.8662 & 750.9367 & 1483.8396 & 742.4235 & 1482.8556 & 741.9314 & 413 \\
\hline 8 & 842.4770 & 421.7422 & 825.4505 & 413.2289 & 824.4665 & 412.7369 & $G$ & 1387.7821 & 694.3947 & 1370.7556 & 685.8814 & 1369.7716 & 685.3894 & 412 \\
\hline 9 & 979.5359 & 490.2716 & 962.5094 & 481.7583 & 961.5254 & 481.2663 & $\mathrm{H}$ & 1330.7607 & 665.8840 & 1313.7341 & 657.3707 & 1312.7501 & 656.8787 & 711 \\
\hline 10 & 1080.5836 & 540.7955 & 1063.5571 & 532.2822 & 1062.5731 & 531.7902 & $T$ & 1193.7018 & 597.3545 & 1176.6752 & 588.8412 & 1175.6912 & 588.3492 & 210 \\
\hline 11 & 1193.6677 & 597.3375 & 1176.6411 & 588.8242 & 1175.6571 & 588.3322 & I & 1092.6541 & 546.8307 & 1075.6275 & 538.3174 & 1074.6435 & 537.8254 & 49 \\
\hline 12 & 1324.7082 & 662.8577 & 1307.6816 & 654.3444 & 1306.6976 & 653.8524 & $M$ & 979.5700 & 490.2886 & 962.5435 & 481.7754 & 961.5595 & 481.2834 & 48 \\
\hline 13 & 1438.7511 & 719.8792 & 1421.7245 & 711.3659 & 1420.7405 & 710.8739 & $\mathrm{~N}$ & 848.5295 & 424.7684 & 831.5030 & 416.2551 & 830.5190 & 415.7631 & 7 \\
\hline 14 & 1551.8352 & 776.4212 & 1534.8086 & 767.9079 & 1533.8246 & 767.4159 & $L$ & 734.4866 & 367.7469 & 717.4601 & 359.2337 & 716.4760 & 358.7417 & 76 \\
\hline 15 & 1664.9192 & 832.9632 & 1647.8927 & 824.4500 & 1646.9087 & 823.9580 & L & 621.4025 & 311.2049 & 604.3760 & 302.6916 & 603.3920 & 302.1996 & 5 \\
\hline 16 & 1763.9876 & 882.4975 & 1746.9611 & 873.9842 & 1745.9771 & 873.4922 & $\mathrm{~V}$ & 508.3185 & 254.6629 & 491.2919 & 246.1496 & 490.3079 & 245.6576 & \begin{tabular}{|l|l|}
6 & 4 \\
\end{tabular} \\
\hline 17 & 1879.0146 & 940.0109 & 1861.9880 & 931.4976 & 1861.0040 & 931.0056 & D & 409.2501 & 205.1287 & 392.2235 & 196.6154 & 391.2395 & 196.1234 & \begin{tabular}{|l|}
4 \\
\end{tabular} \\
\hline 18 & 1992.0986 & 996.5530 & 1975.0721 & 988.0397 & 1974.0881 & 987.5477 & L & 294.2231 & 147.6152 & 277.1966 & 139.1019 & & & 2 \\
\hline 19 & & & & & & & $\mathbf{R}$ & 181.1391 & 91.0732 & 164.1125 & 82.5599 & & & 1 \\
\hline
\end{tabular}
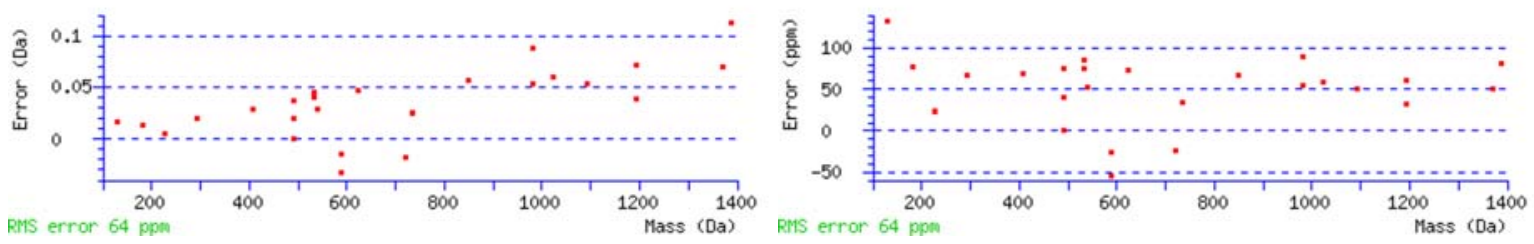


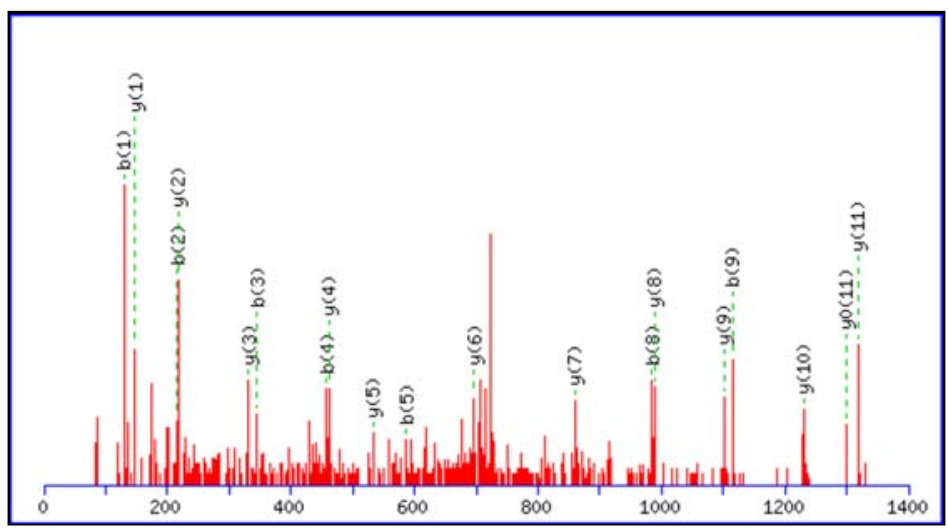

Monoisotopic mass of neutral peptide $\operatorname{Mr}$ (calc): 1444.7271

Ions Score: 74 Expect: $6.4 \mathrm{e}-005$

Matches (Bold Red): 20/116 fragment ions using 32 most intense peaks

\begin{tabular}{|c|c|c|c|c|c|c|c|c|c|c|c|c|c|c|}
\hline \# & b & $b^{++}$ & $b *$ & $\mathrm{~b} *^{++}$ & $b^{0}$ & $b^{0++}$ & Seq. & y & $y^{++}$ & y* & $y *^{++}$ & $y^{0}$ & $y^{0++}$ & $\#$ \\
\hline 1 & 129.1022 & 65.0548 & 112.0757 & 56.5415 & & & K & & & & & & & 12 \\
\hline 2 & 216.1343 & 108.5708 & 199.1077 & 100.0575 & 198.1237 & 99.5655 & $S$ & 1317.6395 & 659.3234 & 1300.6129 & 650.8101 & 1299.6289 & 650.3181 & 11 \\
\hline 3 & 345.1769 & 173.0921 & 328.1503 & 164.5788 & 327.1663 & 164.0868 & $E$ & 1230.6075 & 615.8074 & 1213.5809 & 607.2941 & 1212.5969 & 606.8021 & 10 \\
\hline 4 & 458.2609 & 229.6341 & 441.2344 & 221.1208 & 440.2504 & 220.6288 & I & 1101.5649 & 551.2861 & 1084.5383 & 542.7728 & 1083.5543 & 542.2808 & 9 \\
\hline 5 & 587.3035 & 294.1554 & 570.2770 & 285.6421 & 569.2929 & 285.1501 & $E$ & 988.4808 & 494.7440 & 971.4543 & 486.2308 & 970.4702 & 485.7388 & 8 \\
\hline 6 & 750.3668 & 375.6871 & 733.3403 & 367.1738 & 732.3563 & 366.6818 & $Y$ & 859.4382 & 430.2227 & 842.4117 & 421.7095 & & & 7 \\
\hline 7 & 913.4302 & 457.2187 & 896.4036 & 448.7054 & 895.4196 & 448.2134 & $Y$ & 696.3749 & 348.6911 & 679.3483 & 340.1778 & & & 6 \\
\hline 8 & 984.4673 & 492.7373 & 967.4407 & 484.2240 & 966.4567 & 483.7320 & A & 533.3116 & 267.1594 & 516.2850 & 258.6461 & & & 5 \\
\hline 9 & 1115.5078 & 558.2575 & 1098.4812 & 549.7442 & 1097.4972 & 549.2522 & M & 745 & 231.6409 & 445.2479 & 223.1276 & & & 4 \\
\hline 10 & 1228.5918 & 614.7995 & 1211.5653 & 606.2863 & 1210.5813 & 605.7943 & L & 331.2340 & 166.1206 & 314.2074 & 157.6073 & & & 3 \\
\hline 11 & 1299.6289 & 650.3181 & 1282.6024 & 641.8048 & 1281.6184 & 641.3128 & A & 218.1499 & 109.5786 & 201.1234 & 101.0653 & & & 2 \\
\hline 12 & & & & & & & $\mathrm{~K}$ & 147.1128 & 74.0600 & 130.0863 & 65.5468 & & & 1 \\
\hline
\end{tabular}
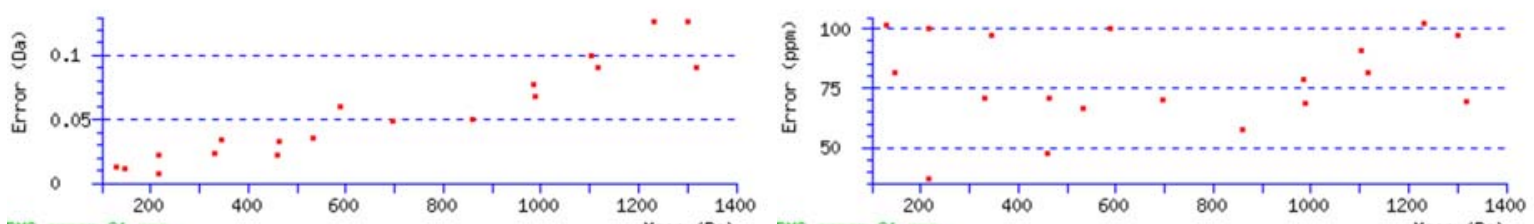

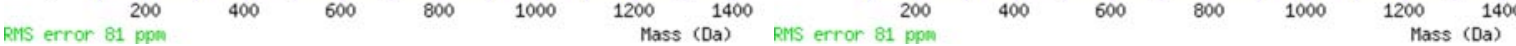




\section{Peptide View}

MS/MS Fragmentation of LGANSLLDLVVFGR

Found in gi|54607098|ref|NP_075770.1|, succinate dehydrogenase Fp subunit [Mus musculus]

Match to Query 459: 1482.862048 from $(742.438300,2+)$

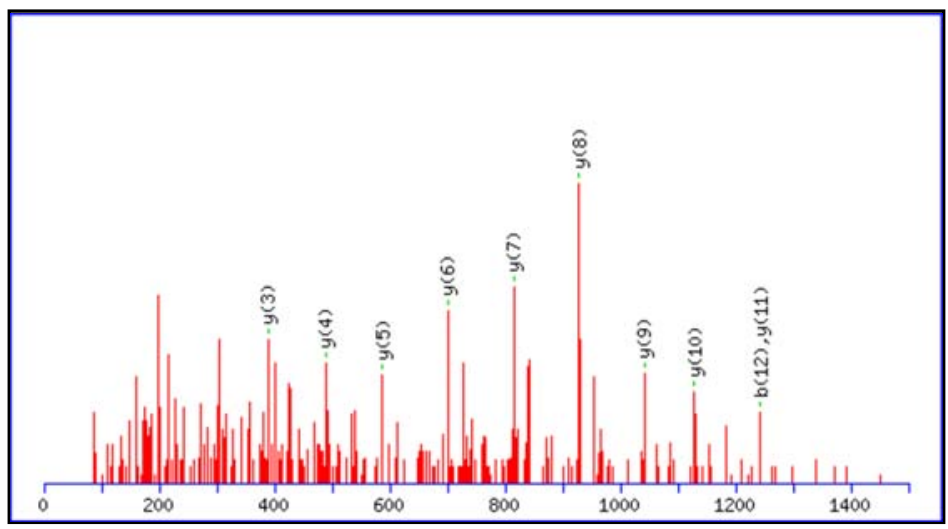

Monoisotopic mass of neutral peptide $\operatorname{Mr}(\mathrm{calc}): 1482.8686$

Variable modifications:

R14 : Arginine-13C615N4 (R-full)

Ions Score: 69 Expect: 0.00024

Matches (Bold Red): 10/130 fragment ions using 18 most intense peaks

\begin{tabular}{|c|c|c|c|c|c|c|c|c|c|c|c|c|c|c|}
\hline$\#$ & b & $b^{++}$ & $b *$ & $b *^{++}$ & $b^{0}$ & $b^{0++}$ & Seq. & $y$ & $y^{++}$ & y* & $y *^{++}$ & $y^{0}$ & $y^{0++}$ & $\#$ \\
\hline 1 & 114.0913 & 57.5493 & & & & & L & & & & & & & 14 \\
\hline 2 & 171.1128 & 86.0600 & & & & & G & 1370.7918 & 685.8996 & 1353.7653 & 677.3863 & 1352.7813 & 676.8943 & 13 \\
\hline 3 & 242.1499 & 121.5786 & & & & & A & 1313.7704 & 657.3888 & 1296.7438 & 648.8756 & 1295.7598 & 648.3835 & 12 \\
\hline 4 & 356.1928 & 178.6001 & 339.1663 & 170.0868 & & & $\mathrm{~N}$ & 1242.7333 & 621.8703 & 1225.7067 & 613.3570 & 1224.7227 & 612.8650 & 11 \\
\hline 5 & 443.2249 & 222.1161 & 426.1983 & 213.6028 & 425.2143 & 213.1108 & $S$ & 1128.6903 & 564.8488 & 1111.6638 & 556.3355 & 1110.6798 & 555.8435 & 10 \\
\hline 6 & 556.3089 & 278.6581 & 539.2824 & 270.1448 & 538.2984 & 269.6528 & L & 1041.6583 & 521.3328 & 1024.6318 & 512.8195 & 1023.6478 & 512.3275 & 9 \\
\hline 7 & 669.3930 & 335.2001 & 652.3664 & 326.6869 & 651.3824 & 326.1948 & L & 928.5743 & 464.7908 & 911.5477 & 456.2775 & 910.5637 & 455.7855 & 8 \\
\hline 8 & 784.4199 & 392.7136 & 767.3934 & 384.2003 & 766.4094 & 383.7083 & D & 815.4902 & 408.2487 & 798.4636 & 399.7355 & 797.4796 & 399.2435 & 7 \\
\hline 9 & 897.5040 & 449.2556 & 880.4774 & 440.7424 & 879.4934 & 440.2503 & $L$ & 700.4633 & 350.7353 & 683.4367 & 342.2220 & & & 6 \\
\hline 10 & 996.5724 & 498.7898 & 979.5458 & 490.2766 & 978.5618 & 489.7846 & V & 587.3792 & 294.1932 & 570.3526 & 285.6800 & & & 5 \\
\hline 11 & 1095.6408 & 548.3240 & 1078.6143 & 539.8108 & 1077.6302 & 539.3188 & V & 488.3108 & 244.6590 & 471.2842 & 236.1458 & & & 4 \\
\hline 12 & 1242.7092 & 621.8582 & 1225.6827 & 613.3450 & \begin{tabular}{|l|}
1224.6987 \\
\end{tabular} & 612.8530 & $\mathrm{~F}$ & 389.2424 & 195.1248 & 372.2158 & 186.6116 & & & 3 \\
\hline 13 & 1299.7307 & 650.3690 & 1282.7041 & 641.8557 & 1281.7201 & 641.3637 & G & 242.1740 & 121.5906 & 225.1474 & 113.0773 & & & 2 \\
\hline 14 & & & & & & & $R$ & 185.1525 & 93.0799 & 168.1260 & 84.5666 & & & 1 \\
\hline
\end{tabular}
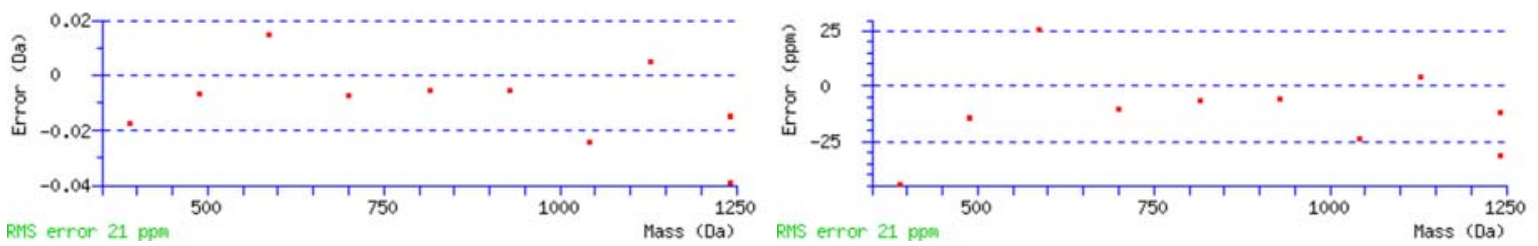

RMS error 21 ppe

Mass (Da)

RMS error 21 ppm

lass (Da) 


\section{Peptide View}

MS/MS Fragmentation of LIEEVHAVVTVR

Found in gi|38348472|ref|NP_941015.1|, adaptor-related protein complex 2, sigma 1 subunit [Mus musculus]

Match to Query 362: 1363.826848 from(682.920700,2+)

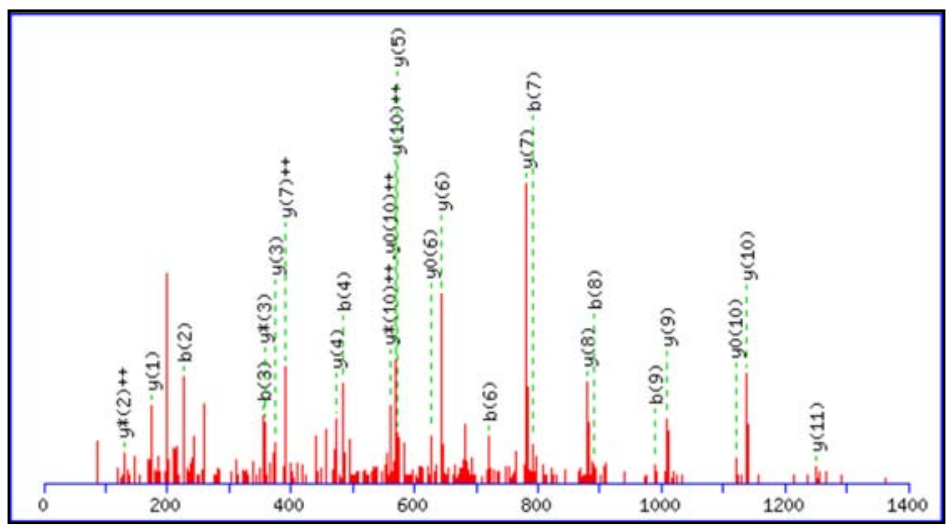

Monoisotopic mass of neutral peptide $\operatorname{Mr}(\mathrm{calc}): 1363.7823$

Ions Score: 61 Expect: 0.0014

Matches (Bold Red): 25/102 fragment ions using 39 most intense peaks

\begin{tabular}{|c|c|c|c|c|c|c|c|c|c|c|c|c|}
\hline 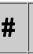 & b & $b^{++}$ & $b^{0}$ & $b^{0++}$ & Seq. & y & $y^{++}$ & y* & $y *^{++}$ & $y^{0}$ & $y^{0++}$ & \# \\
\hline T & 4.0913 & 3 & & & $L$ & & & & & & & 12 \\
\hline 2 & 227.1754 & 114.0913 & & & I & & 64 & 0 & & 950 & 11 & 11 \\
\hline 3 & 356.2180 & 178.6126 & 38.2074 & 73 & $\mathrm{E}$ & 1138.6215 & 569.8144 & 1121.5949 & 561.3011 & 1120.6109 & 560.8091 & 10 \\
\hline 4 & 6 & 1339 & 0 & 23 & $\mathrm{E}$ & 89 & 31 & 23 & 40 & 91.5683 & 878 & \\
\hline 5 & 584.3290 & 292.6681 & 566.3184 & 328 & V & 363 & 440.7718 & 863.5097 & 432.2585 & 862.5257 & 431.7665 & 8 \\
\hline 6 & 721.3879 & 361.1976 & 703. & 23 & $\mathrm{H}$ & & 391.2376 & & & 763.4573 & 382 & \\
\hline 7 & 792.4250 & 396.7161 & 774. & 38 & A & & 81 & 24 & 31 & 626. & 31 & 6 \\
\hline 8 & 891.4 & 446.2503 & 873.4829 & 437.2451 & V & 9 & 287.1896 & 55 & 278 & 555.3613 & 278 & 5 \\
\hline 9 & 990.5618 & 495 & 972.5 & & V & & 237.6554 & & & 456.2929 & 228.6501 & 4 \\
\hline 10 & 1091.6095 & 5 & 1073.5 & 53 & $T$ & 0 & 12 & 358. & 17 & 357.2245 & 179. & 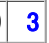 \\
\hline 11 & 1190.6779 & 595.8426 & 1172.6674 & 586.8373 & V & 1874 & 137.5973 & 608 & 129.0840 & & & 2 \\
\hline 2 & & & & & $\mathrm{R}$ & 175.1190 & 3.0631 & 158.0924 & 79.5498 & & & \\
\hline
\end{tabular}
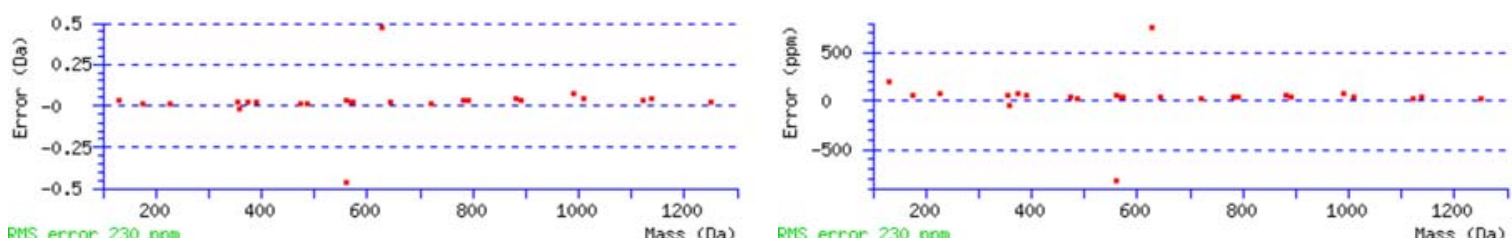


\section{Peptide View}

MS/MS Fragmentation of LIGQIVSSITASLR

Found in gi|6755901|ref|NP_035783.1|, tubulin, alpha 1 [Mus musculus]

Match to Query 297: 1456.936648 from(729.475600,2+)

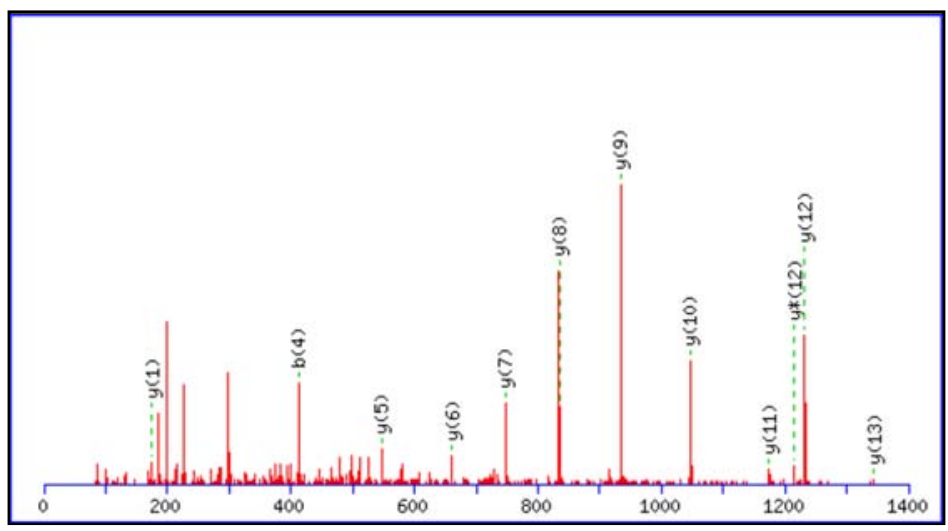

Monoisotopic mass of neutral peptide $\operatorname{Mr}$ (calc): 1456.8613

Ions Score: 93 Expect: 1.1e-006

Matches (Bold Red): 12/134 fragment ions using 20 most intense peaks

\begin{tabular}{|c|c|c|c|c|c|c|c|c|c|c|c|c|c|c|}
\hline$\#$ & b & $b^{++}$ & $b *$ & $b *^{++}$ & $b^{0}$ & $b^{0++}$ & Seq. & y & $y^{++}$ & y* & $y *^{++}$ & $y^{0}$ & $y^{0++}$ & $\#$ \\
\hline 1 & 114.0913 & 57.5493 & & & & & L & & & & & & & 14 \\
\hline 2 & 227.1754 & 114.0913 & & & & & I & 1344.7845 & 672.8959 & 1327.7580 & 664.3826 & 1326.7740 & 663.8906 & 13 \\
\hline 3 & 284.1969 & 142.6021 & & & & & G & 1231.7005 & 616.3539 & 1214.6739 & 607.8406 & 1213.6899 & 607.3486 & 12 \\
\hline 4 & 412.2554 & 206.6314 & 395.2289 & 198.1181 & & & $Q$ & 1174.6790 & 587.8431 & 1157.6525 & 579.3299 & 1156.6684 & 578.8379 & 11 \\
\hline 5 & 525.3395 & 263.1734 & 508.3129 & 254.6601 & & & I & 1046.6204 & 523.8138 & 1029.5939 & 515.3006 & 1028.6099 & 514.8086 & 10 \\
\hline 6 & 624.4079 & 312.7076 & 607.3814 & 304.1943 & & & V & 933.5364 & 467.2718 & 916.5098 & 458.7585 & 915.5258 & 458.2665 & 9 \\
\hline 7 & 711.4399 & 356.2236 & 694.4134 & 347.7103 & 693.4294 & 347.2183 & $S$ & 834.4680 & 417.7376 & 817.4414 & 409.2243 & 816.4574 & 408.7323 & 8 \\
\hline 8 & 798.4720 & 399.7396 & 781.4454 & 391.2263 & 780.4614 & 390.7343 & $S$ & 747.4359 & 374.2216 & 730.4094 & 365.7083 & 729.4254 & 365.2163 & 7 \\
\hline 9 & 911.5560 & 456.2817 & 894.5295 & 447.7684 & 893.5455 & 447.2764 & I & 660.4039 & 330.7056 & 643.3773 & 322.1923 & 642.3933 & 321.7003 & 6 \\
\hline 10 & 1012.6037 & 506.8055 & 995.5772 & 498.2922 & 994.5931 & 497.8002 & $T$ & 547.3198 & 274.1636 & 530.2933 & 265.6503 & 529.3093 & 265.1583 & 5 \\
\hline 11 & 1083.6408 & 542.3240 & 1066.6143 & 533.8108 & 1065.6303 & 533.3188 & A & 446.2722 & 223.6397 & 429.2456 & 215.1264 & 428.2616 & 214.6344 & 4 \\
\hline 12 & 1170.6728 & 585.8401 & 1153.6463 & 577.3268 & 1152.6623 & 576.8348 & $S$ & 375.2350 & 188.1212 & 358.2085 & 179.6079 & 357.2245 & 179.1159 & 3 \\
\hline 13 & 1283.7569 & 642.3821 & 1266.7304 & 633.8688 & 1265.7463 & 633.3768 & L & 288.2030 & 144.6051 & 271.1765 & 136.0919 & & & 2 \\
\hline 14 & & & & & & & $R$ & 175.1190 & 88.0631 & 158.0924 & 79.5498 & & & 1 \\
\hline
\end{tabular}
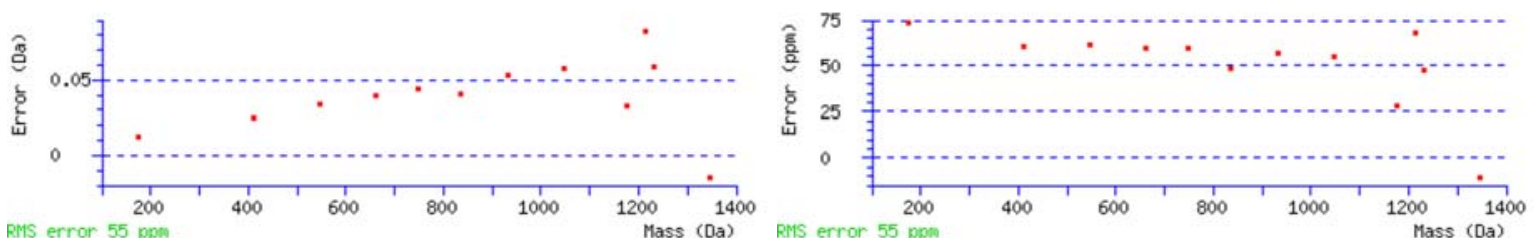

RMS error 55 ppo 


\section{Peptide View}

MS/MS Fragmentation of LLQDSVDFSLADAINTEFK

Found in gi|31982755|ref|NP_035831.2|, vimentin [Mus musculus]

Match to Query 760: 2125.234248 from $\left(1063.624400,2^{+}\right)$

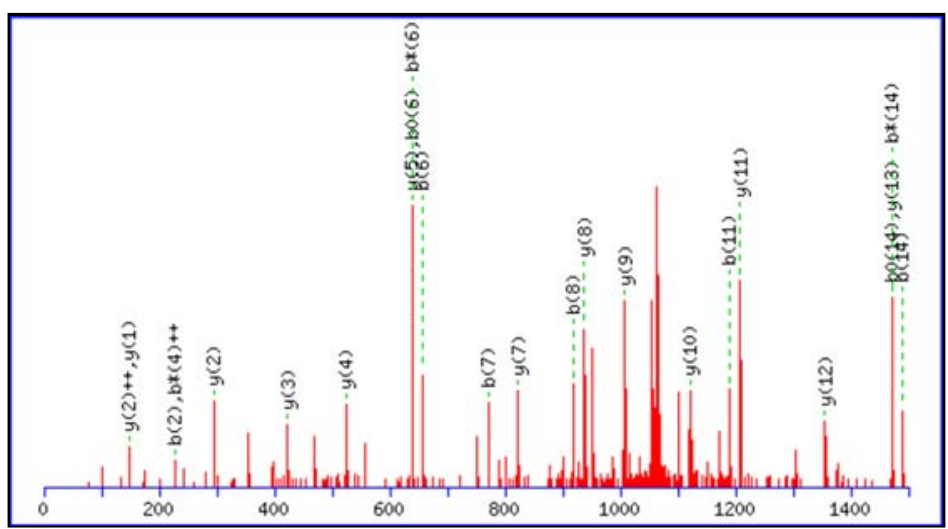

Monoisotopic mass of neutral peptide $\mathrm{Mr}$ (calc): 2125.0579

Ions Score: 105 Expect: $1.5 \mathrm{e}-007$

Matches (Bold Red) : 24/202 fragment ions using 27 most intense peaks

\begin{tabular}{|c|c|c|c|c|c|c|c|c|c|c|c|c|c|c|}
\hline \# & b & $b^{++}$ & $b *$ & $b *^{++}$ & $b^{0}$ & $b^{0++}$ & Seq. & $y$ & $y^{++}$ & $y *$ & $y *^{++}$ & $y^{0}$ & $y^{0++}$ & $\#$ \\
\hline 1 & 114.0913 & 57.5493 & & & & & L & & & & & & & 19 \\
\hline 2 & 227.1754 & 114.0913 & & & & & L & 2012.9811 & 1006.9942 & 1995.9545 & 998.4809 & 1994.9705 & 997.9889 & 18 \\
\hline 3 & 355.2340 & 178.1206 & 338.2074 & 169.6074 & & & $Q$ & 1899.8970 & 950.4522 & 1882.8705 & 941.9389 & 1881.8865 & 941.4469 & 17 \\
\hline 4 & 470.2609 & 235.6341 & 453.2344 & 227.1208 & 452.2504 & 226.6288 & D & 1771.8385 & 886.4229 & 1754.8119 & 877.9096 & 1753.8279 & 877.4176 & 16 \\
\hline 5 & 557.2929 & 279.1501 & 540.2664 & 270.6368 & 539.2824 & 270.1448 & s & 1656.8115 & 828.9094 & 1639.7850 & 820.3961 & 1638.8009 & 819.9041 & 15 \\
\hline 6 & 656.3614 & 328.6843 & 639.3348 & 320.1710 & 638.3508 & 319.6790 & V & 1569.7795 & 785.3934 & 1552.7529 & 776.8801 & 1551.7689 & 776.3881 & 14 \\
\hline 7 & 771.3883 & 386.1978 & 754.3617 & 377.6845 & 753.3777 & 377.1925 & D & 1470.7111 & 735.8592 & 1453.6845 & 727.3459 & 1452.7005 & 726.8539 & 13 \\
\hline 8 & 918.4567 & 459.7320 & 901.4302 & 451.2187 & 900.4461 & 450.7267 & $\mathrm{~F}$ & 1355.6841 & 678.3457 & 1338.6576 & 669.8324 & 1337.6736 & 669.3404 & 12 \\
\hline 9 & 1005.4887 & 503.2480 & 988.4622 & 494.7347 & 987.4782 & 494.2427 & s & 1208.6157 & 604.8115 & 1191.5892 & 596.2982 & 1190.6052 & 595.8062 & 11 \\
\hline 10 & 1118.5728 & 559.7900 & 1101.5462 & 551.2768 & 1100.5622 & 550.7848 & L & 1121.5837 & 561.2955 & 1104.5571 & 552.7822 & 1103.5731 & 552.2902 & 10 \\
\hline 11 & 1189.6099 & 595.3086 & 1172.5834 & 586.7953 & 1171.5993 & 586.3033 & A & 1008.4996 & 504.7535 & 991.4731 & 496.2402 & 990.4891 & 495.7482 & 9 \\
\hline 12 & 1304.6368 & 652.8221 & 1287.6103 & 644.3088 & 1286.6263 & 643.8168 & D & 937.4625 & 469.2349 & 920.4360 & 460.7216 & 919.4520 & 460.2296 & 8 \\
\hline 13 & 1375.6740 & 688.3406 & 1358.6474 & 679.8273 & 1357.6634 & 679.3353 & A & 822.4356 & 411.7214 & 805.4090 & 403.2082 & 804.4250 & 402.7161 & 7 \\
\hline 14 & 1488.7580 & 744.8826 & 1471.7315 & 736.3694 & 1470.7475 & 735.8774 & I & 751.3985 & 376.2029 & 734.3719 & 367.6896 & 733.3879 & 367.1976 & 6 \\
\hline 15 & 1602.8009 & 801.9041 & \begin{tabular}{|l|}
1585.7744 \\
\end{tabular} & \begin{tabular}{|l|}
793.3908 \\
\end{tabular} & 1584.7904 & 792.8988 & $N$ & 638.3144 & 319.6608 & 621.2879 & 311.1476 & 620.3038 & 310.6556 & 5 \\
\hline 16 & 1703.8486 & 852.4280 & 1686.8221 & 843.9147 & 1685.8381 & 843.4227 & $T$ & 524.2715 & 262.6394 & 507.2449 & 254.1261 & 506.2609 & 253.6341 & 4 \\
\hline 17 & 1832.8912 & 916.9492 & 1815.8647 & 908.4360 & 1814.8807 & 907.9440 & $E$ & 423.2238 & 212.1155 & 406.1973 & 203.6023 & 405.2132 & 203.1103 & 3 \\
\hline 18 & 1979.9596 & 990.4835 & 1962.9331 & 981.9702 & 1961.9491 & 981.4782 & $\mathrm{~F}$ & 294.1812 & 147.5942 & 277.1547 & 139.0810 & & & 2 \\
\hline 19 & & & & & & & $\mathrm{~K}$ & 147.1128 & 74.0600 & 130.0863 & 65.5468 & & & 1 \\
\hline
\end{tabular}
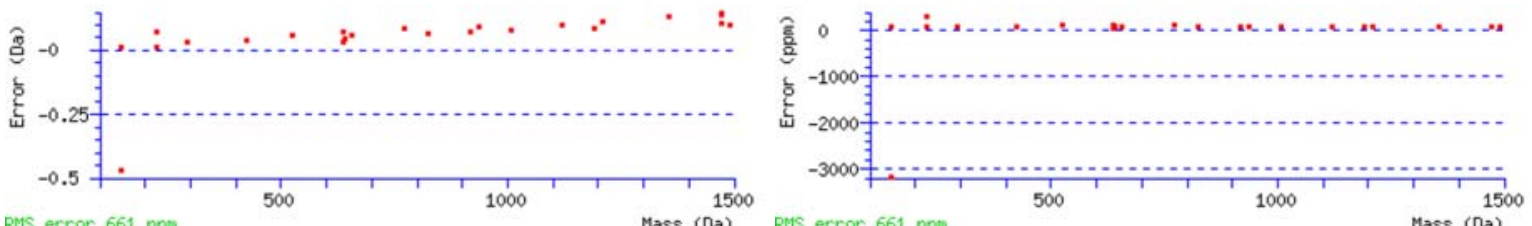

RMS error $661 \mathrm{ppm}$ Mass (Da) RMS error 661 ppm Mass (Da) 


\section{Peptide View}

MS/MS Fragmentation of LMLLLEVISGER

Found in gi|11230802|ref|NP_068695.1|, actinin alpha 4 [Mus musculus]

Match to Query 368: 1371.844448 from(686.929500,2+)

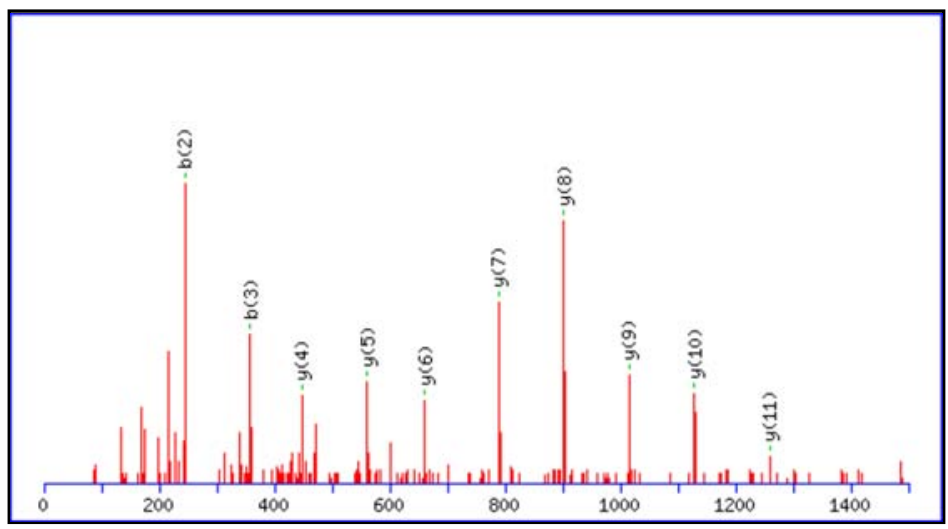

Monoisotopic mass of neutral peptide $\operatorname{Mr}$ (calc): 1371.7795

Ions Score: 69 Expect: 0.0002

Matches (Bold Red): 10/98 fragment ions using 20 most intense peaks

\begin{tabular}{|c|c|c|c|c|c|c|c|c|c|c|c|c|}
\hline \# & b & $b^{++}$ & $b^{0}$ & $b^{0++}$ & Seq. & $\mathbf{y}$ & $y^{++}$ & y* & $y *^{++}$ & $y^{0}$ & $y^{0++}$ & \# \\
\hline 1 & 114.0913 & 57.5493 & & & L & & & & & & & 12 \\
\hline 2 & 245.1318 & 123.0695 & & & $M$ & 1259.7028 & 630.3550 & 1242.6762 & 621.8417 & 1241.6922 & 621.3497 & 11 \\
\hline 3 & 358.2159 & 179.6116 & & & L & 1128.6623 & 564.8348 & 1111.6357 & 556.3215 & 1110.6517 & 555.8295 & 10 \\
\hline 4 & 471.2999 & 236.1536 & & & L & 1015.5782 & 508.2927 & 998.5517 & 499.7795 & 997.5676 & 499.2875 & 9 \\
\hline 5 & 584.3840 & 292.6956 & & & L & 902.4942 & 451.7507 & 885.4676 & 443.2374 & 884.4836 & 442.7454 & 8 \\
\hline 6 & 713.4266 & 357.2169 & 695.4160 & 348.2116 & $E$ & 789.4101 & 395.2087 & 772.3835 & 386.6954 & 771.3995 & 386.2034 & 7 \\
\hline 7 & 812.4950 & 406.7511 & 794.4844 & 397.7459 & V & 660.3675 & 330.6874 & 643.3410 & 322.1741 & 642.3569 & 321.6821 & 6 \\
\hline 8 & 925.5791 & 463.2932 & 907.5685 & 454.2879 & I & 561.2991 & 281.1532 & 544.2725 & 272.6399 & 543.2885 & 272.1479 & 5 \\
\hline 9 & 1012.6111 & 506.8092 & 994.6005 & 497.8039 & $\mathrm{~s}$ & 448.2150 & 224.6112 & 431.1885 & 216.0979 & 430.2045 & 215.6059 & 4 \\
\hline 10 & 1069.6325 & 535.3199 & 1051.6220 & 526.3146 & G & 361.1830 & 181.0951 & 344.1565 & 172.5819 & 343.1724 & 172.0899 & 3 \\
\hline 11 & 1198.6751 & 599.8412 & 1180.6646 & 590.8359 & $E$ & 304.1615 & 152.5844 & 287.1350 & 144.0711 & 286.1510 & 143.5791 & 2 \\
\hline 12 & & & & & $\mathrm{R}$ & 175.1190 & 88.0631 & 158.0924 & 79.5498 & & & 1 \\
\hline
\end{tabular}
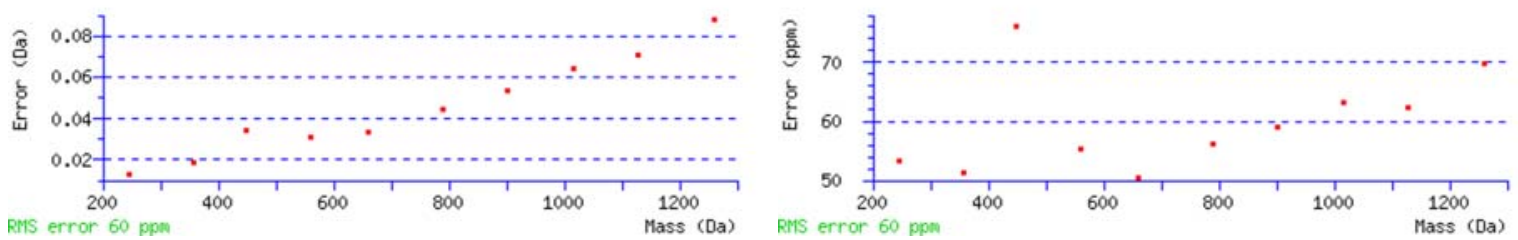


\section{Peptide View}

MS/MS Fragmentation of LNYAQWYPIVVFLNPDSK

Found in gi|6678355|ref|NP_033412.1|, tight junction protein 1 [Mus musculus]

Match to Query 532: 2166.216248 from $(1084.115400,2+)$

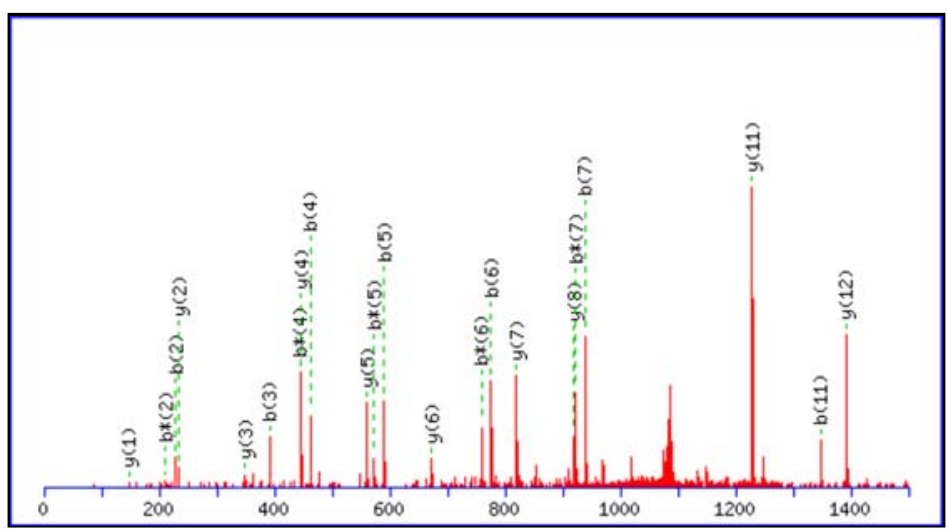

Monoisotopic mass of neutral peptide $\operatorname{Mr}$ (calc): 2166. 1149

Ions Score: 54 Expect: 0.018

Matches (Bold Red): 22/170 fragment ions using 42 most intense peaks

\begin{tabular}{|c|c|c|c|c|c|c|c|c|c|c|c|c|c|c|}
\hline$\#$ & b & $b^{++}$ & $b *$ & $b *^{++}$ & $b^{0}$ & $b^{0++}$ & Seq. & $y$ & $\mathrm{y}^{++}$ & y* & $y *^{++}$ & $y^{0}$ & $y^{0++}$ & $\#$ \\
\hline 1 & 114.0913 & 57.5493 & & & & & L & & & & & & & 18 \\
\hline 2 & 228.1343 & 114.5708 & 211.1077 & 106.0575 & & & $\mathrm{~N}$ & 2054.0382 & 1027.5227 & 2037.0116 & 1019.0094 & 42036.0276 & 1018.5174 & 17 \\
\hline 3 & 391.1976 & 196.1024 & 374.1710 & 187.5892 & & & $\mathrm{Y}$ & 1939.9952 & 970.5013 & 1922.9687 & 961.9880 & 1921.9847 & \begin{tabular}{l|l}
7 & 961.4960
\end{tabular} & 16 \\
\hline 4 & 462.2347 & 231.6210 & 445.2082 & 223.1077 & & & A & 1776.9319 & 888.9696 & 1759.9054 & 880.4563 & 31758.9213 & 879.9643 & 15 \\
\hline 5 & 590.2933 & 295.6503 & 573.2667 & 287.1370 & & & $Q$ & 1705.8948 & 853.4510 & 1688.8682 & 844.9378 & 31687.8842 & 844.4458 & 314 \\
\hline 6 & 776.3726 & 388.6899 & 759.3460 & 380.1767 & & & W & 1577.8362 & 789.4217 & 1560.8097 & 780.9085 & 51559.8256 & \begin{tabular}{|l|l}
5 & 780.4165
\end{tabular} & 513 \\
\hline 7 & 939.4359 & 470.2216 & 922.4094 & 461.7083 & & & $Y$ & 1391.7569 & 696.3821 & 1374.7304 & 687.8688 & 81373.7463 & $\begin{array}{ll}687.3768 \\
\end{array}$ & 12 \\
\hline 8 & 1036.4887 & 518.7480 & 1019.4621 & 510.2347 & & & $\mathrm{P}$ & 1228.6936 & 614.8504 & 1211.6670 & 606.3371 & 1210.6830 & 605.8451 & 11 \\
\hline 9 & 1149.5727 & 575.2900 & 1132.5462 & 566.7767 & & & I & 1131.6408 & 566.3240 & 1114.6143 & 557.8108 & 81113.6302 & $\begin{array}{ll}257.3188 \\
\end{array}$ & 10 \\
\hline 10 & 1248.6412 & 624.8242 & 1231.6146 & 616.3109 & & & V & 1018.5568 & 509.7820 & 1001.5302 & 501.2687 & 71000.5462 & 500.7767 & 9 \\
\hline 11 & 1347.7096 & 674.3584 & 1330.6830 & 665.8451 & & & V & 919.4883 & 460.2478 & 902.4618 & 451.7345 & \begin{tabular}{|l|l}
5 & 901.4778
\end{tabular} & \begin{tabular}{|l|l|}
3 & 451.2425
\end{tabular} & 8 \\
\hline 12 & 1494.7780 & 747.8926 & 1477.7514 & 739.3794 & & & $\mathrm{~F}$ & 820.4199 & 410.7136 & 803.3934 & 402.2003 & $\begin{array}{l}3802.4094 \\
\end{array}$ & $4 \quad 401.7083$ & 3 \\
\hline 13 & 1607.8620 & 804.4347 & 1590.8355 & 795.9214 & & & L & 673.3515 & 337.1794 & 656.3250 & 328.6661 & $1 \quad 655.3410$ & 328.1741 & 6 \\
\hline 14 & 1721.9050 & 861.4561 & 1704.8784 & 852.9428 & & & $\mathrm{~N}$ & 560.2675 & 280.6374 & 543.2409 & 272.1241 & $1 \quad 542.2569$ & 271.6321 & 5 \\
\hline 15 & 1818.9577 & 909.9825 & 1801.9312 & 901.4692 & & & $P$ & 446.2245 & 223.6159 & 429.1980 & 215.1026 & \begin{tabular}{|l|l}
6 & 428.2140 \\
\end{tabular} & 214.6106 & 4 \\
\hline 16 & 1933.9847 & 967.4960 & 1916.9581 & 958.9827 & 1915.9741 & 958.4907 & D & 349.1718 & 175.0895 & 332.1452 & 166.5762 & $2 \quad 331.1612$ & $\begin{array}{l}166.0842 \\
\end{array}$ & 3 \\
\hline 17 & 2021.0167 & 1011.0120 & 2003.9901 & 1002.4987 & 2003.0061 & 1002.0067 & $S$ & 234.1448 & 117.5761 & 217.1183 & 109.0628 & 3216.1343 & \begin{tabular}{|l|l}
3 & 108.5708 \\
\end{tabular} & 32 \\
\hline 18 & & & & & & & $\mathrm{~K}$ & 147.1128 & 74.0600 & 130.0863 & 65.5468 & & & 1 \\
\hline
\end{tabular}
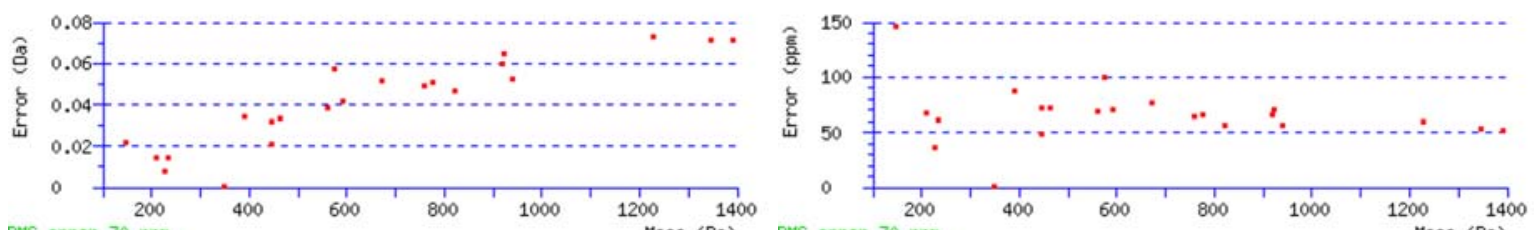

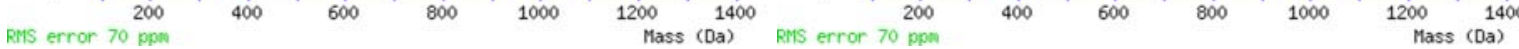




\section{Peptide View}

MS/MS Fragmentation of LPAAGVGDMVMATVK

Found in gi|12584986|ref|NP_075029.1|, ribosomal protein L23 [Mus musculus]

Match to Query 298: 1458.823048 from $(730.418800,2+)$

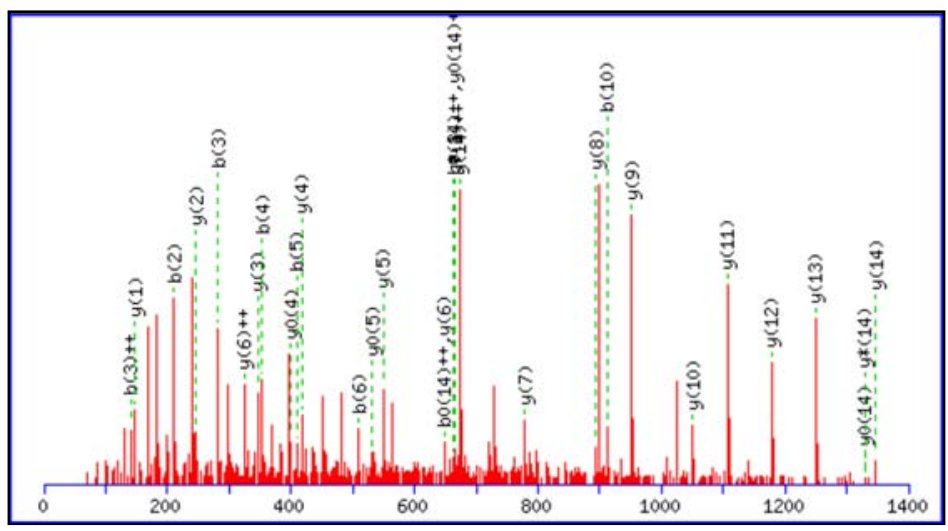

Monoisotopic mass of neutral peptide $\operatorname{Mr}$ (calc): 1458.7574

Ions Score: 70 Expect: 0.00019

Matches (Bold Red): 31/122 fragment ions using 93 most intense peaks

\begin{tabular}{|c|c|c|c|c|c|c|c|c|c|c|c|c|}
\hline \# & b & $\mathrm{b}^{++}$ & $b^{0}$ & $b^{0++}$ & Seq. & $y$ & $y^{++}$ & y* & $y *^{++}$ & $y^{0}$ & $y^{0++}$ & $\#$ \\
\hline 1 & 114.0913 & 57.5493 & & & $\mathrm{~L}$ & & & & & & & 15 \\
\hline 2 & 211.1441 & 106.0757 & & & $P$ & 1346.6806 & 673.8439 & 1329.6541 & 665.3307 & 1328.6701 & 664.8387 & 14 \\
\hline 3 & 282.1812 & 141.5942 & & & $A$ & 1249.6279 & 625.3176 & 1232.6013 & 616.8043 & 1231.6173 & 616.3123 & 13 \\
\hline 4 & 353.2183 & 177.1128 & & & A & 1178.5908 & 589.7990 & 1161.5642 & 581.2857 & 1160.5802 & 580.7937 & 72 \\
\hline 5 & 410.2398 & 205.6235 & & & G & 1107.5536 & 554.2805 & 1090.5271 & 545.7672 & 1089.5431 & 545.2752 & 211 \\
\hline 6 & 509.3082 & 255.1577 & & & V & 1050.5322 & \begin{tabular}{|l|}
525.7697 \\
\end{tabular} & 1033.5056 & 517.2565 & 1032.5216 & 516.7644 & 10 \\
\hline 7 & 566.3296 & 283.6685 & & & G & 951.4638 & 476.2355 & 934.4372 & 467.7222 & 933.4532 & 467.2302 & 2 \\
\hline 8 & 681.3566 & 341.1819 & 663.3460 & 332.1766 & D & 894.4423 & \begin{tabular}{|l|}
447.7248 \\
\end{tabular} & 877.4158 & 439.2115 & 876.4317 & 438.7195 & 0 \\
\hline 9 & 812.3971 & 406.7022 & 794.3865 & 397.6969 & $M$ & 779.4154 & 390.2113 & 762.3888 & 381.6980 & 761.4048 & 381.2060 & 7 \\
\hline 10 & 911.4655 & 456.2364 & 893.4549 & 447.2311 & V & 648.3749 & 324.6911 & 631.3483 & 316.1778 & \begin{tabular}{|r|} 
\\
\end{tabular} & 315.6858 & 3 \\
\hline 11 & 1042.5060 & 521.7566 & 1024.4954 & 512.7513 & $M$ & 549.3065 & 275.1569 & 532.2799 & 266.6436 & 531.2959 & 266.1516 & $\hat{0}$ \\
\hline 12 & 1113.5431 & 557.2752 & 1095.5325 & 548.2699 & A & 418.2660 & 209.6366 & 401.2395 & 201.1234 & 400.2554 & 200.6314 & $\begin{array}{l}4 \quad 4 \\
\end{array}$ \\
\hline 13 & 1214.5907 & 607.7990 & 1196.5802 & 598.7937 & $T$ & 347.2289 & \begin{tabular}{|l|}
174.1181 \\
\end{tabular} & 330.2023 & 165.6048 & \begin{tabular}{|l|}
329.2183 \\
\end{tabular} & 165.1128 & 3 \\
\hline 14 & 1313.6592 & 657.3332 & 1295.6486 & 648.3279 & $\mathrm{~V}$ & 246.1812 & 123.5942 & 229.1547 & 115.0810 & & & 2 \\
\hline 15 & & & & & $\mathrm{~K}$ & 147.1128 & 74.0600 & 130.0863 & 65.5468 & & & 1 \\
\hline
\end{tabular}
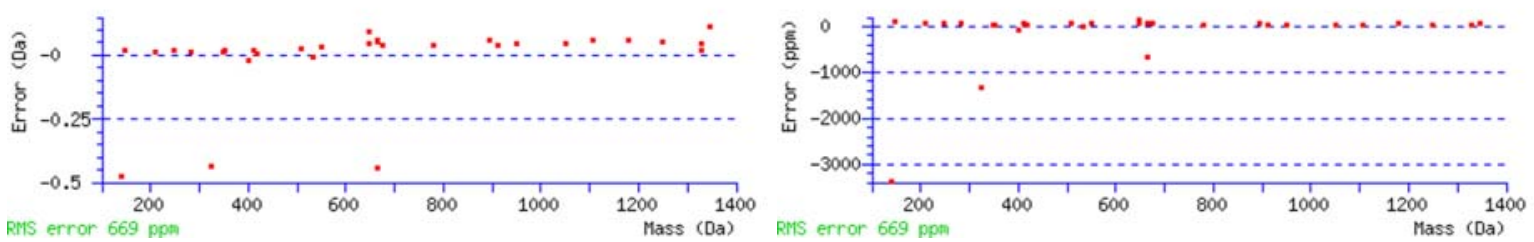


\section{Peptide View}

MS/MS Fragmentation of LSLQLALTELETLAEK

Found in gi|40254212|ref|NP_766003.2|, Rho guanine nucleotide exchange factor (GEF) 10-like [Mus musculus]

Match to Query 594: 1771.020048 from $(886.517300,2+)$

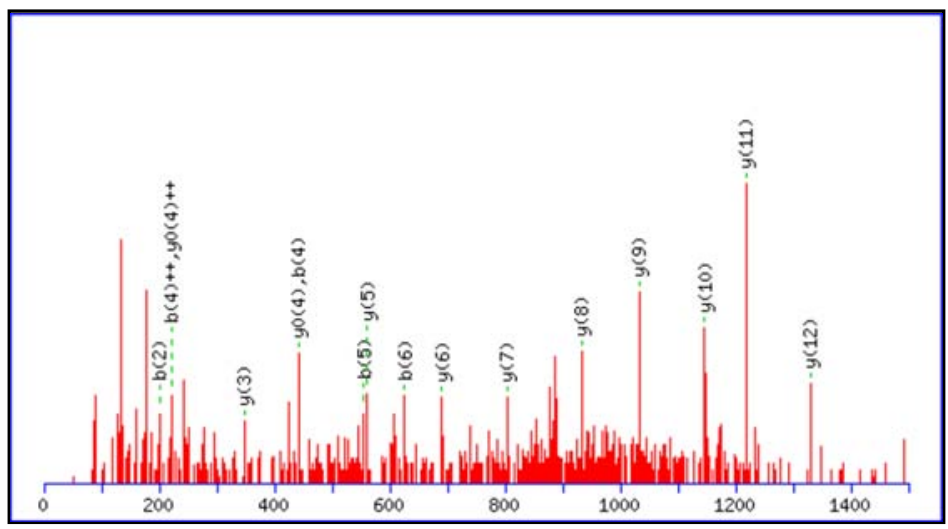

Monoisotopic mass of neutral peptide $\operatorname{Mr}(\mathrm{calc}): 1770.9978$

Ions Score: 66 Expect: 0.00081

Matches (Bold Red): 16/170 fragment ions using 27 most intense peaks

\begin{tabular}{|c|c|c|c|c|c|c|c|c|c|c|c|c|c|c|}
\hline \# & b & $b^{++}$ & $b *$ & $b *^{++}$ & $b^{0}$ & $b^{0++}$ & Seq. & $y$ & $y^{++}$ & $y *$ & $y *^{++}$ & $y^{0}$ & $y^{0++}$ & $\#$ \\
\hline 1 & 114.0913 & 57.5493 & & & & & L & & & & & & & 16 \\
\hline 2 & 201.1234 & 101.0653 & & & 183.1128 & 92.0600 & 5 & 1658.9211 & 829.9642 & 1641.8945 & 821.4509 & 1640.9105 & 820.9589 & 15 \\
\hline 3 & 314.2074 & 157.6074 & & & 296.1969 & 148.6021 & L & 1571.8890 & 786.4482 & 1554.8625 & 777.9349 & 1553.8785 & 5777.4429 & 14 \\
\hline 4 & 442.2660 & 221.6366 & 425.2395 & 213 & 424.2554 & 212.6314 & $Q$ & 145 & 729.9061 & 1441.7784 & 721 & 1440.7944 & 4720.9008 & 13 \\
\hline 5 & 555.3501 & 278.1787 & 538.3235 & 269.6654 & 537.3395 & 269.1734 & L & 1330.7464 & 665.8768 & 1313.7198 & 657.3636 & 1312.7358 & 656.8716 & 12 \\
\hline 6 & 626.3872 & 313.6972 & 609.3606 & 305.1840 & 608.3766 & 304.6919 & A & 1217.6623 & 609.3348 & 1200.6358 & 600.8215 & 1199.6518 & 600.3295 & 11 \\
\hline 7 & 739.4712 & 370.2393 & 722.4447 & 361.7260 & 721.4607 & 361.2340 & L & 1146.6252 & 573.8162 & 1129.5987 & 565.3030 & 1128.6147 & 564.8110 & 10 \\
\hline 8 & 840.5189 & 420.7631 & 823.4924 & 412.2498 & 822.5084 & 411.7578 & $T$ & 1033.5412 & 517.2742 & 1016.5146 & 508.7609 & 1015.5306 & 508.2689 & 9 \\
\hline 9 & 969.5615 & 485.2844 & 952.5350 & 476.7711 & 951.5509 & 476.2791 & $E$ & 932.4935 & 466.7504 & 915.4669 & 458.2371 & 914.4829 & 457.7451 & 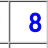 \\
\hline 10 & 1082.6456 & 541.8264 & 1065.6190 & 533.3131 & 1064.6350 & 532.8211 & L & 803.4509 & 402.2291 & 786.4243 & 393.7158 & 785.4403 & 393.2238 & 7 \\
\hline 11 & 1211.6882 & 606.3477 & 1194.6616 & 597.8344 & 1193.6776 & 597.3424 & $\mathrm{E}$ & 690.3668 & 345.6871 & 673.3403 & 337.1738 & 672.3563 & 336.6818 & 6 \\
\hline 12 & 1312.7358 & 656.8716 & 1295.7093 & 648.3583 & 1294.7253 & 647.8663 & $T$ & 561.3242 & 281.1658 & 544.2977 & 272.6525 & 543.3137 & 272.1605 & 5 \\
\hline 13 & 1425.8199 & 713.4136 & 1408.7933 & 704.9003 & 1407.8093 & 704.4083 & $\mathrm{~L}$ & 460.2766 & 230.6419 & 443.2500 & 222.1286 & 442.2660 & 221.6366 & 4 \\
\hline 14 & 1496.8570 & 748.9321 & 1479.8305 & 740.4189 & 1478.8464 & 739.9269 & A & 347.1925 & 174.0999 & 330.1660 & 165.5866 & 329.1819 & 165.0946 & 3 \\
\hline 15 & 1625.8996 & 813.4534 & 1608.8730 & 804.9402 & 1607.8890 & 804.4482 & $\mathrm{E}$ & 276.1554 & 138.5813 & 259.1288 & 130.0681 & 258.1448 & 129.5761 & 2 \\
\hline 16 & & & & & & & $\mathrm{~K}$ & 147.1128 & 74.0600 & 130.0863 & 65.5468 & & & 1 \\
\hline
\end{tabular}
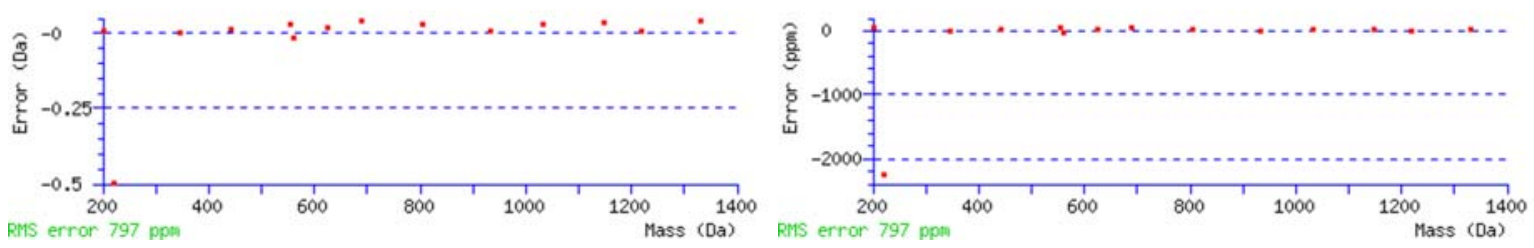


\section{Peptide View}

MS/MS Fragmentation of LVLSPADMAAVFINLEDLIK

Found in gi|6678555|ref|NP_033526.1|, Vav2 oncogene [Mus musculus]

Match to Query 702: 2171.237448 from(1086.626000,2+)

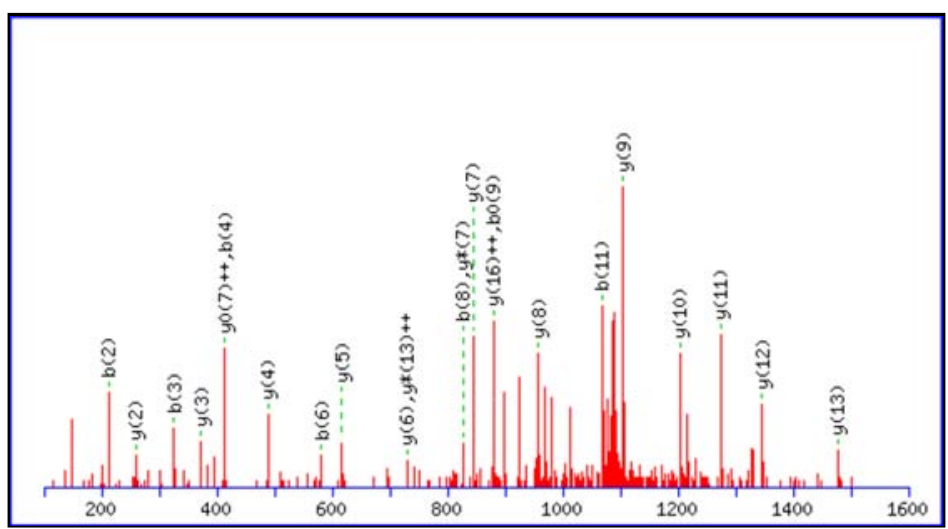

Monoisotopic mass of neutral peptide $\operatorname{Mr}(\mathrm{calc}): 2171.1911$

Ions Score: 98 Expect: $7.6 \mathrm{e}-007$

Matches (Bold Red): 23/190 fragment ions using 25 most intense peaks

\begin{tabular}{|c|c|c|c|c|c|c|c|c|c|c|c|c|c|c|}
\hline$\#$ & b & $b^{++}$ & $b *$ & $b *^{++}$ & $b^{0}$ & $b^{0++}$ & Seq. & $y$ & $y^{++}$ & $y *$ & $y *^{++}$ & $y^{0}$ & $y^{0++}$ & \# \\
\hline 1 & 114.0913 & 57.5493 & & & & & L & & & & & & & 20 \\
\hline 2 & 213.1597 & 107.0835 & & & & & V & 2059.1143 & 1030.0608 & 2042.0878 & 1021.5475 & 2041.1038 & 1021.0555 & 19 \\
\hline 3 & 326.2438 & 163.6255 & & & & & L & 1960.0459 & 980.5266 & 1943.0194 & 972.0133 & 1942.0354 & 971.5213 & 18 \\
\hline 4 & 413.2758 & 207.1416 & & & 395.2653 & 198.1363 & $\mathrm{~S}$ & 1846.9619 & 923.9846 & 1829.9353 & 915.4713 & 1828.9513 & 914.9793 & 17 \\
\hline 5 & 510.3286 & 255.6679 & & & 492.3180 & 246.6627 & $\mathbf{P}$ & 1759.9298 & 880.4686 & 1742.9033 & 871.9553 & 1741.9193 & 871.4633 & 16 \\
\hline 6 & 581.3657 & 291.1865 & & & 563.3551 & 282.1812 & A & 1662.8771 & 831.9422 & 1645.8505 & 823.4289 & 1644.8665 & 822.9369 & 15 \\
\hline 7 & 696.3926 & 348.7000 & & & 678.3821 & 339.6947 & D & 1591.8400 & 796.4236 & 1574.8134 & 787.9103 & 1573.8294 & 787.4183 & 14 \\
\hline 8 & 827.4331 & 414.2202 & & & 809.4226 & 405.2149 & M & 1476.8130 & 738.9101 & 1459.7865 & 730.3969 & 1458.8025 & 729.9049 & 13 \\
\hline 9 & 898.4702 & 449.7388 & & & 880.4597 & 440.7335 & A & 1345.7725 & 673.3899 & 1328.7460 & 664.8766 & 1327.7620 & 664.3846 & 12 \\
\hline 10 & 969.5073 & 485.2573 & & & 951.4968 & 476.2520 & A & 1274.7354 & 637.8714 & 1257.7089 & 629.3581 & 1256.7249 & 628.8661 & 11 \\
\hline 11 & 1068.5758 & 534.7915 & & & 1050.5652 & 525.7862 & V & 1203.6983 & 602.3528 & 1186.6718 & 593.8395 & 1185.6878 & 593.3475 & 10 \\
\hline 12 & 1215.6442 & 608.3257 & & & 1197.6336 & 599.3204 & $\mathrm{~F}$ & 1104.6299 & 552.8186 & 1087.6034 & 544.3053 & 1086.6193 & 543.8133 & 9 \\
\hline 13 & 1328.7282 & 664.8678 & & & 1310.7177 & 655.8625 & I & 957.5615 & 479.2844 & 940.5350 & 470.7711 & 939.5509 & 470.2791 & 8 \\
\hline 14 & 1442.7712 & 721.8892 & 1425.7446 & 713.3759 & 1424.7606 & 712.8839 & $\mathrm{~N}$ & 844.4774 & 422.7424 & 827.4509 & 414.2291 & 826.4669 & 413.7371 & 7 \\
\hline 15 & 1555.8552 & 778.4312 & 1538.8287 & 769.9180 & 1537.8447 & 769.4260 & L & 730.4345 & 365.7209 & 713.4080 & 357.2076 & 712.4239 & 356.7156 & 6 \\
\hline 16 & 1684.8978 & 842.9525 & 1667.8713 & 834.4393 & 1666.8872 & 833.9473 & $E$ & 617.3505 & 309.1789 & 600.3239 & 300.6656 & 599.3399 & 300.1736 & 5 \\
\hline 17 & 1799.9247 & 900.4660 & 1782.8982 & 891.9527 & 1781.9142 & 891.4607 & D & 488.3079 & 244.6576 & 471.2813 & 236.1443 & 470.2973 & 235.6523 & 4 \\
\hline 18 & 1913.0088 & 957.0080 & 1895.9823 & 948.4948 & 1894.9982 & 948.0028 & L & 373.2809 & 187.1441 & 356.2544 & 178.6308 & & & 3 \\
\hline 19 & 2026.0929 & 1013.5501 & 2009.0663 & 1005.0368 & 2008.0823 & 1004.5448 & I & 260.1969 & 130.6021 & 243.1703 & 122.0888 & & & 2 \\
\hline 20 & & & & & & & $\mathrm{~K}$ & 147.1128 & 74.0600 & 130.0863 & 65.5468 & & & 1 \\
\hline
\end{tabular}
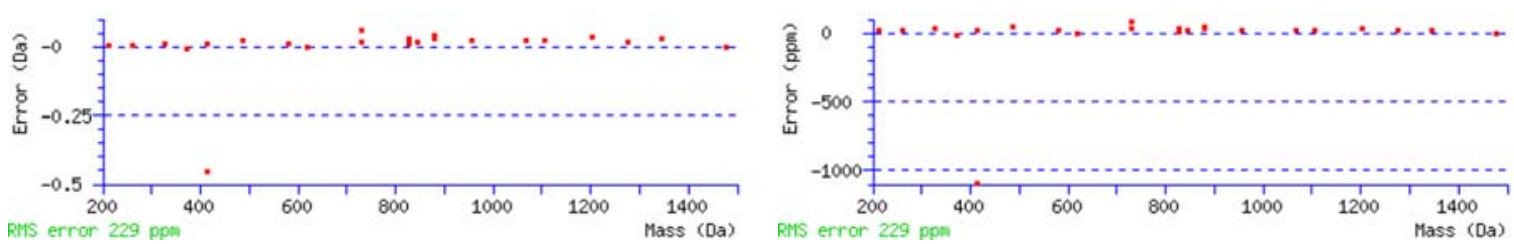


\section{Peptide View}

MS/MS Fragmentation of LYTLVTYVPVTTFK

Found in gi|94398865|ref|XP_996947.1|, PREDICTED: similar to ribosomal protein L31 [Mus musculus]

Match to Query 941: 1643.990048 from(823.002300,2+)

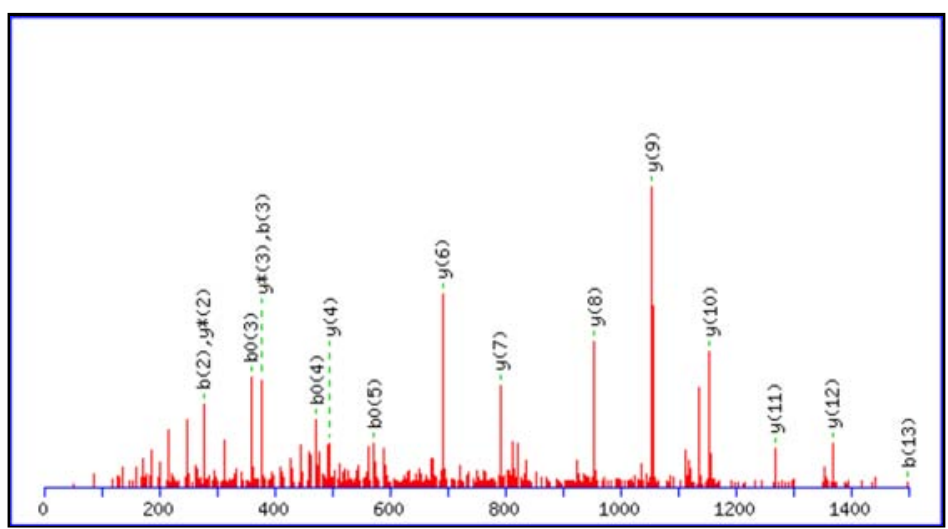

Monoisotopic mass of neutral peptide $\mathrm{Mr}$ (calc): 1643.9174

Ions Score: 54 Expect: 0.011

Matches (Bold Red): 16/122 fragment ions using 36 most intense peaks

\begin{tabular}{|c|c|c|c|c|c|c|c|c|c|c|c|c|}
\hline \# & b & $b^{++}$ & $b^{0}$ & $b^{0++}$ & Seq. & $y$ & $y^{++}$ & y* & $y *^{++}$ & $y^{0}$ & $y^{0++}$ & $\#$ \\
\hline 1 & 114.0913 & 57.5493 & & & L & & & & & & & 14 \\
\hline 2 & 277.1547 & 139.0810 & & & $Y$ & 1531.8406 & 766.4240 & 1514.8141 & 757.9107 & 1513.8301 & 757.4187 & 13 \\
\hline 3 & 378.2023 & 189.6048 & 360.1918 & 180.5995 & T & 1368.7773 & 684.8923 & 1351.7508 & 676.3790 & 1350.7667 & 675.8870 & 12 \\
\hline 4 & 491.2864 & 246.1468 & 473.2758 & 237.1416 & L & 1267.7296 & 634.3685 & 1250.7031 & 625.8552 & 1249.7191 & 625.3632 & 11 \\
\hline 5 & 590.3548 & 295.6810 & 572.3443 & 286.6758 & V & 1154.6456 & 577.8264 & 1137.6190 & 569.3131 & 1136.6350 & 568.8211 & 10 \\
\hline 6 & 691.4025 & 346.2049 & 673.3919 & 337.1996 & $T$ & 1055.5772 & 528.2922 & 1038.5506 & 519.7789 & 1037.5666 & 519.2869 & . \\
\hline 7 & 854.4658 & 427.7366 & 836.4553 & 418.7313 & $Y$ & 954.5295 & 477.7684 & 937.5029 & 469.2551 & 936.5189 & 468.7631 & 8 \\
\hline 8 & 953.5342 & 477.2708 & 935.5237 & 468.2655 & V & 791.4662 & 396.2367 & 774.4396 & 387.7234 & 773.4556 & 387.2314 & 7 \\
\hline 9 & 1050.5870 & 525.7971 & 1032.5764 & 516.7919 & $\mathrm{P}$ & 692.3977 & 346.7025 & 675.3712 & 338.1892 & 674.3872 & 337.6972 & 6 \\
\hline 10 & 1149.6554 & 575.3313 & 1131.6448 & 566.3261 & V & 595.3450 & 298.1761 & 578.3184 & 289.6629 & 577.3344 & 289.1708 & 5 \\
\hline 11 & 1250.7031 & 625.8552 & 1232.6925 & 616.8499 & $T$ & 496.2766 & 248.6419 & 479.2500 & 240.1286 & 478.2660 & 239.6366 & 4 \\
\hline 12 & 1351.7508 & 676.3790 & 1333.7402 & 667.3737 & $T$ & 395.2289 & 198.1181 & 378.2023 & 189.6048 & 377.2183 & 189.1128 & 3 \\
\hline 13 & 1498.8192 & 749.9132 & 1480.8086 & 740.9079 & $\mathrm{~F}$ & 294.1812 & 147.5942 & 277.1547 & 139.0810 & & & 2 \\
\hline 14 & & & & & $\mathrm{~K}$ & 147.1128 & 74.0600 & 130.0863 & 65.5468 & & & -1 \\
\hline
\end{tabular}
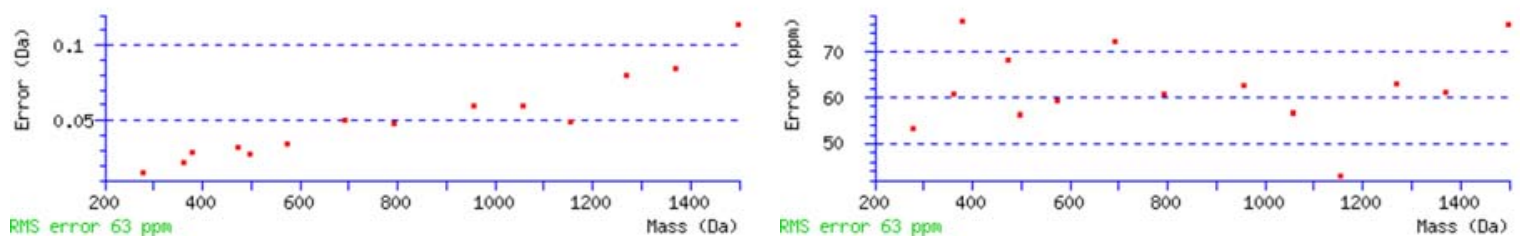


\section{Peptide View}

MS/MS Fragmentation of MLGSPVDSVLFYAITTLHNLLLHQEGAK

Found in gi|6671684|ref|NP_031640.1|, catenin (cadherin associated protein), beta 1, 88kDa [Mus musculus]

Match to Query 865: 3066.676096 from $(767.676300,4+)$

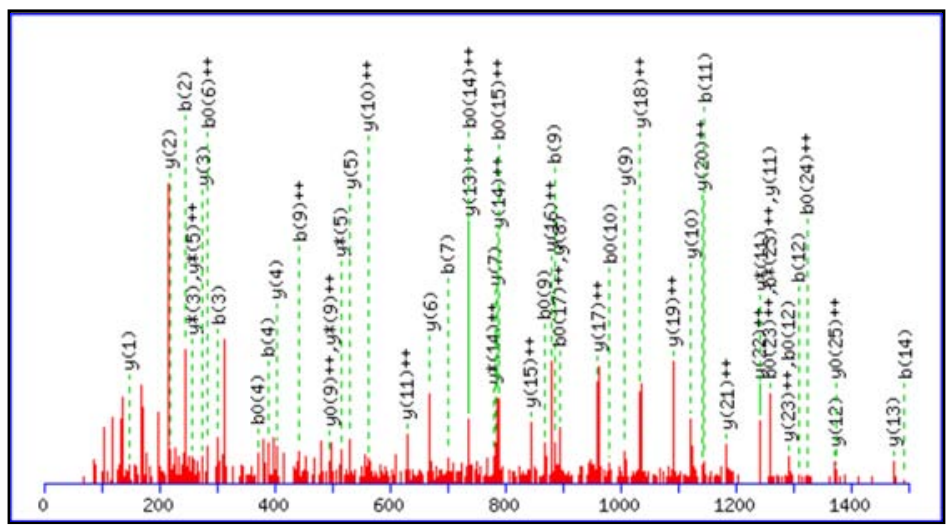

Monoisotopic mass of neutral peptide $\operatorname{Mr}(\mathrm{calc}): 3066.6211$

Ions Score: 68 Expect: 0.0016

Matches (Bold Red): 54/276 fragment ions using 134 most intense peaks

\begin{tabular}{|c|c|c|c|c|c|c|c|c|c|c|c|c|c|c|}
\hline \# & b & $b^{++}$ & $b *$ & $\mathrm{~b} *^{++}$ & $b^{0}$ & $b^{0++}$ & Seq. & y & $y^{++}$ & y* & $y *^{++}$ & $y^{0}$ & $y^{0++}$ & $\#$ \\
\hline 1 & 132.0478 & 66.5275 & & & & & M & & & & & & & 28 \\
\hline 2 & 245.1318 & 123.0695 & & & & & L & 2936.5880 & 1468.7976 & 2919.5614 & 1460.2843 & 2918.5774 & 1459.7923 & 327 \\
\hline 3 & 302.1533 & 151.5803 & & & & & $G$ & 2823.5039 & 1412.2556 & 2806.4774 & 1403.7423 & 2805.4933 & 1403.2503 & 326 \\
\hline 4 & 389.1853 & 195.0963 & & & 371.1747 & 186.0910 & $S$ & 2766.4824 & 1383.7449 & 2749.4559 & 1375.2316 & 2748.4719 & 1374.7396 & 625 \\
\hline 5 & 486.2381 & 243.6227 & & & 468.2275 & 234.6174 & $P$ & 2679.4504 & 1340.2288 & 2662.4239 & 1331.7156 & 2661.4398 & 1331.2236 & 624 \\
\hline 6 & 585.3065 & 293.1569 & & & 567.2959 & 284.1516 & V & 2582.3977 & 1291.7025 & 2565.3711 & 1283.1892 & 2564.3871 & 1282.6972 & 23 \\
\hline 7 & 700.3334 & 350.6703 & & & 682.3229 & 341.6651 & D & 2483.3292 & 1242.1683 & 2466.3027 & 1233.6550 & 2465.3187 & 1233.1630 & 22 \\
\hline 8 & 787.3654 & 394.1864 & & & 769.3549 & 385.1811 & $\mathrm{~s}$ & 2368.3023 & 1184.6548 & 2351.2758 & 1176.1415 & 2350.2917 & 1175.6495 & 521 \\
\hline 9 & 886.4339 & 443.7206 & & & 868.4233 & 434.7153 & V & 2281.2703 & 1141.1388 & 2264.2437 & 1132.6255 & 2263.2597 & 1132.1335 & 520 \\
\hline 10 & 999.5179 & 500.2626 & & & 981.5074 & 491.2573 & $\mathrm{~L}$ & 2182.2019 & 1091.6046 & 2165.1753 & 1083.0913 & 2164.1913 & 1082.5993 & 319 \\
\hline 111 & 1146.5863 & 573.7968 & & & 1128.5758 & 564.7915 & $\mathrm{~F}$ & 2069.1178 & 1035.0625 & 2052.0913 & 1026.5493 & 2051.1072 & 1026.0573 & 318 \\
\hline 121 & 1309.6497 & 655.3285 & & & 1291.6391 & 646.3232 & $Y$ & 1922.0494 & 961.5283 & 1905.0228 & 953.0151 & 1904.0388 & 952.5231 & 17 \\
\hline \begin{tabular}{|l|}
13 \\
\end{tabular} & 1380.6868 & 690.8470 & & & 1362.6762 & 681.8417 & A & 1758.9861 & \begin{tabular}{|l|}
879.9967 \\
\end{tabular} & 1741.9595 & 871.4834 & 1740.9755 & 870.9914 & 416 \\
\hline 141 & 1493.7708 & 747.3891 & & & 1475.7603 & 738.3838 & I & 1687.9490 & 844.4781 & 1670.9224 & 835.9648 & 1669.9384 & 835.4728 & 815 \\
\hline 15 & 1594.8185 & 797.9129 & & & 1576.8079 & 788.9076 & $T$ & 1574.8649 & 787.9361 & 1557.8383 & 779.4228 & 1556.8543 & 778.9308 & 814 \\
\hline 16 & 1695.8662 & 848.4367 & & & 1677.8556 & 839.4314 & $T$ & 1473.8172 & 737.4122 & 1456.7907 & 728.8990 & 1455.8066 & 728.4070 & 13 \\
\hline \begin{tabular}{|l|}
17 \\
\end{tabular} & 1808.9502 & 904.9788 & & & 1790.9397 & 895.9735 & L & 1372.7695 & 686.8884 & 1355.7430 & 678.3751 & 1354.7590 & 677.8831 & 112 \\
\hline 18 & 1946.0092 & 973.5082 & & & 1927.9986 & 964.5029 & $\mathrm{H}$ & 1259.6855 & 630.3464 & 1242.6589 & 621.8331 & 1241.6749 & 621.3411 & 11 \\
\hline 19 & 2060.0521 & 1030.5297 & 2043.0255 & 1022.0164 & 2042.0415 & 1021.5244 & $\mathrm{~N}$ & 1122.6266 & 561.8169 & 1105.6000 & 553.3036 & 1104.6160 & 552.8116 & 610 \\
\hline 20 & 2173.1361 & 1087.0717 & 2156.1096 & 1078.5584 & 2155.1256 & 1078.0664 & L & 1008.5836 & 504.7955 & 991.5571 & 496.2822 & 990.5731 & 495.7902 & 9 \\
\hline 21 & 2286.2202 & 1143.6137 & 2269.1937 & 1135.1005 & 2268.2096 & 1134.6085 & L & 895.4996 & 448.2534 & 878.4730 & 439.7401 & 877.4890 & 439.2481 & 8 \\
\hline 22 & 2399.3043 & 1200.1558 & 2382.2777 & 1191.6425 & 2381.2937 & 1191.1505 & $\mathrm{~L}$ & 782.4155 & 391.7114 & 765.3890 & 383.1981 & 764.4049 & 382.7061 & 7 \\
\hline 23 & 2536.3632 & 1268.6852 & 2519.3366 & 1260.1720 & 2518.3526 & 1259.6799 & $\mathrm{H}$ & 669.3315 & 335.1694 & 652.3049 & 326.6561 & 651.3209 & 326.1641 & 16 \\
\hline 24 & 2664.4218 & 133 & 2647.3952 & 1324.2012 & 2646.4112 & 1323 & $Q$ & 532.2725 & 266.6399 & 515.2460 & 258.1266 & 514.2620 & 257.6346 & 65 \\
\hline 25 & 2793.4643 & 1397.2358 & 2776.4378 & 1388.7225 & 2775.4538 & 1388.2305 & $E$ & 404.2140 & 202.6106 & 387.1874 & 194.0973 & 386.2034 & 193.6053 & \begin{tabular}{|l|l}
3 & 4 \\
\end{tabular} \\
\hline 26 & 2850.4858 & 1425.7465 & 2833.4593 & 1417.2333 & 2832.4752 & 1416.7413 & G & 275.1714 & 138.0893 & 258.1448 & 129.5760 & & & 3 \\
\hline 27 & 2921.5229 & 1461.2651 & 2904.4964 & 1452.7518 & 2903.5124 & 1452.2598 & A & 218.1499 & 109.5786 & 201.1234 & 101.0653 & & & 2 \\
\hline 28 & & & & & & & $\mathrm{~K}$ & 147.1128 & 74.0600 & 130.0863 & 65.5468 & & & 1 \\
\hline
\end{tabular}

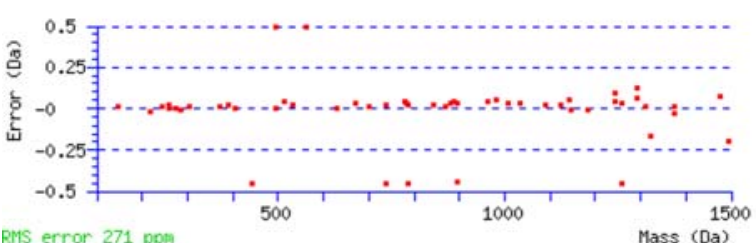

RMS error 271 ppm

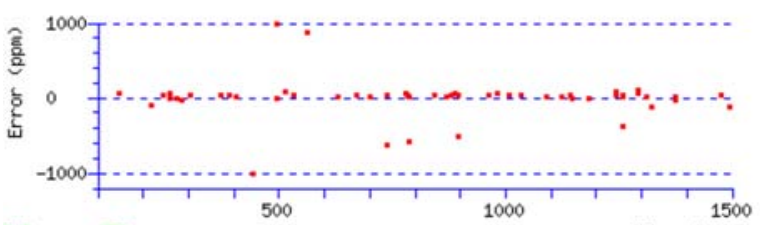

Mass (Da) 


\section{Peptide View}

MS/MS Fragmentation of MNFANVFIGANPLAVDLLEK

Found in gi|10092590|ref|NP_036081.1|, mitogen activated protein kinase 14 [Mus musculus]

Match to Query 1164: 2175.267848 from(1088.641200,2+)

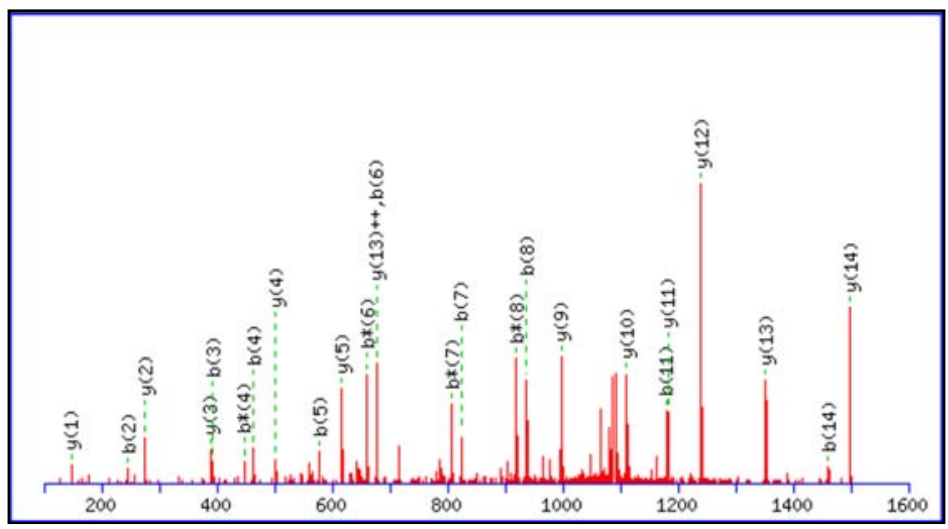

Monoisotopic mass of neutral peptide $\operatorname{Mr}$ (calc): 2175. 1397

Ions Score: 100 Expect: $5.1 \mathrm{e}-007$

Matches (Bold Red) : 25/194 fragment ions using 31 most intense peaks

\begin{tabular}{|c|c|c|c|c|c|c|c|c|c|c|c|c|c|c|}
\hline \# & b & $b^{++}$ & $b *$ & $\mathrm{~b} *^{++}$ & $b^{0}$ & $b^{0++}$ & Seq. & y & $\mathrm{y}^{++}$ & y* & $y *^{++}$ & $y^{0}$ & $y^{0++}$ & \# \\
\hline 1 & 132.0478 & 66.5275 & & & & & $M$ & & & & & & & 20 \\
\hline 2 & 246.0907 & 123.5490 & 229.0641 & 115.0357 & & & $\mathrm{~N}$ & 2045.1066 & 1023.0569 & 2028.0800 & 1014.5436 & 2027.0960 & 1014.0516 & 19 \\
\hline 3 & 393.1591 & 197.0832 & 376.1325 & 188.5699 & & & $\mathrm{~F}$ & 1931.0636 & 966.0354 & 1914.0371 & 957.5222 & 21913.0531 & 957.0302 & 218 \\
\hline 4 & 464.1962 & 232.6017 & 447.1697 & 224.0885 & & & A & 1783.9952 & 892.5012 & 1766.9687 & 883.9880 & 1765.9846 & 883.4960 & 17 \\
\hline 5 & 578.2391 & 289.6232 & 561.2126 & 281.1099 & & & $\mathrm{~N}$ & 1712.9581 & 856.9827 & 1695.9316 & 848.4694 & 41694.9475 & 847.9774 & +16 \\
\hline 6 & 677.3075 & 339.1574 & 660.2810 & 330.6441 & & & V & 1598.9152 & 799.9612 & 1581.8886 & 791.4479 & 91580.9046 & 790.9559 & 15 \\
\hline 7 & 824.3760 & 412.6916 & 807.3494 & 404.1783 & & & $\mathrm{~F}$ & 1499.8468 & 750.4270 & 1482.8202 & 741.9137 & 1481.8362 & 741.4217 & 14 \\
\hline 8 & 937.4600 & 469.2336 & 920.4335 & 460.7204 & & & I & 1352.7784 & 676.8928 & 1335.7518 & 668.3795 & $\begin{array}{ll}5 & 1334.7678 \\
\end{array}$ & 667.8875 & 13 \\
\hline 9 & 994.4815 & | 497.7444 & 977.4549 & 489.2311 & & & G & 1239.6943 & 620.3508 & 1222.6677 & 611.8375 & 51221.6837 & 611.3455 & 12 \\
\hline 10 & 1065.5186 & 533.2629 & 1048.4920 & 524.7497 & & & A & 1182.6728 & 591.8401 & 1165.6463 & 583.3268 & 1164.6623 & 582.8348 & 11 \\
\hline 11 & 1179.5615 & 590.2844 & 1162.5350 & 581.7711 & & & $\mathrm{~N}$ & 1111.6357 & 556.3215 & 1094.6092 & 547.8082 & 21093.6252 & 547.3162 & 10 \\
\hline 12 & 1276.6143 & 638.8108 & 1259.5877 & 630.2975 & & & $P$ & 997.5928 & 499.3000 & 980.5662 & 490.7868 & 979.5822 & 490.2948 & 9 \\
\hline 13 & 1389.6983 & 695.3528 & 1372.6718 & 686.8395 & & & L & 900.5400 & 450.7737 & 883.5135 & 442.2604 & $4 \quad 882.5295$ & 441.7684 & 8 \\
\hline 14 & 1460.7354 & 730.8714 & 1443.7089 & 722.3581 & & & A & 787.4560 & 394.2316 & 770.4294 & 385.7183 & $\begin{array}{ll}3 & 769.4454 \\
\end{array}$ & 385.2263 & 3 \\
\hline 15 & 1559.8039 & 780.4056 & 1542.7773 & 771.8923 & & & V & 716.4189 & 358.7131 & 699.3923 & 350.1998 & \begin{tabular}{l|l}
8 & 698.4083 \\
\end{tabular} & 349.7078 & 6 \\
\hline 16 & 1674.8308 & 837.9190 & 1657.8042 & 829.4058 & 1656.8202 & 828.9138 & $\mathrm{D}$ & 617.3505 & 309.1789 & 600.3239 & 300.6656 & $\begin{array}{l}699.3399 \\
\end{array}$ & 300.1736 & 5 \\
\hline 17 & 1787.9149 & 894.4611 & 1770.8883 & 885.9478 & 1769.9043 & 885.4558 & L & 502.3235 & 251.6654 & 485.2970 & 243.1521 & $1 \quad 484.3129$ & 242.6601 & 4 \\
\hline 18 & 1900.9989 & 951.0031 & 1883.9724 & 942.4898 & 1882.9884 & 941.9978 & $L$ & 389.2395 & 195.1234 & 372.2129 & 186.6101 & 1371.2289 & 186.1181 & 3 \\
\hline 19 & 2030.0415 & 1015.5244 & 2013.0150 & 1007.0111 & 2012.0309 & 1006.5191 & E & 276.1554 & 138.5813 & 259.1288 & 130.0681 & $1 \quad 258.1448$ & 129.5761 & 2 \\
\hline 20 & & & & & & & $\mathrm{~K}$ & 147.1128 & 74.0600 & 130.0863 & 65.5468 & & & 1 \\
\hline
\end{tabular}

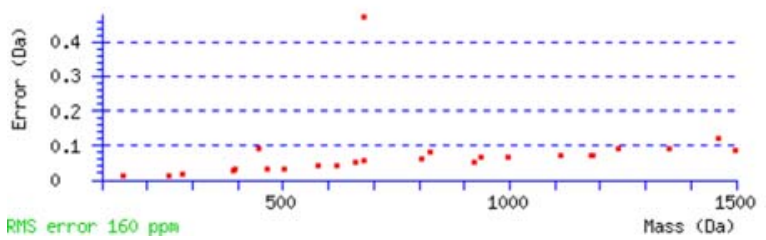

RMS error 160 ppm

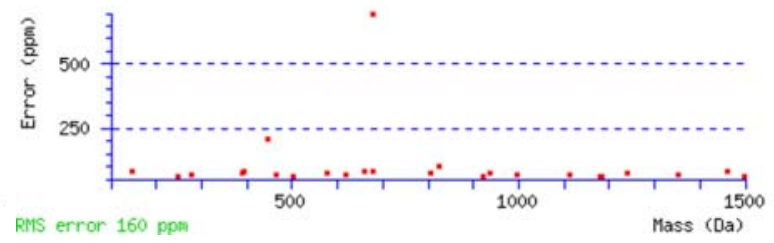




\section{Peptide View}

MS/MS Fragmentation of MQSLTLDVLGTSELLLAK

Found in gi|94419105|ref|XP_001005781.1|, PREDICTED: similar to Development and differentiation-enhancing factor 2 (Pyk2 C-terminus associate

Match to Query 466: 1931.148448 from(966.581500,2+)

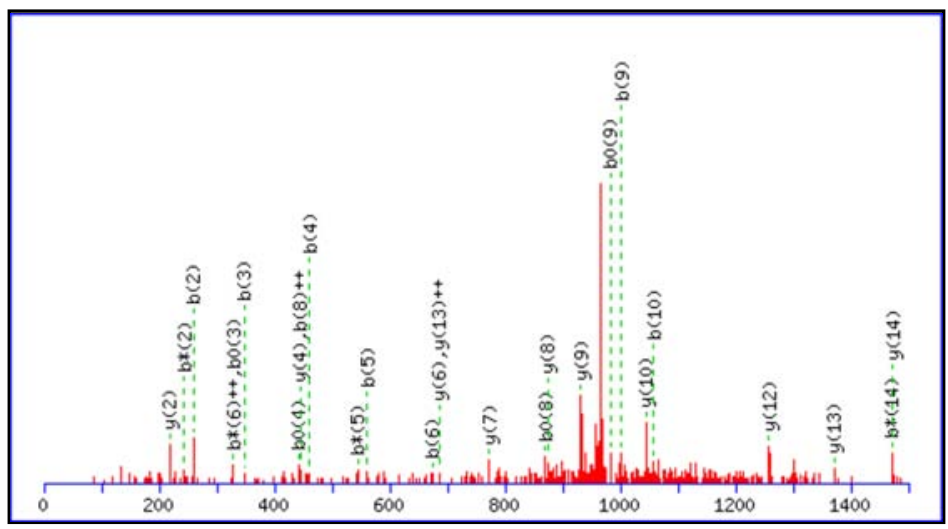

Monoisotopic mass of neutral peptide $\operatorname{Mr}(\mathrm{calc}): 1931.0649$

Ions Score: 50 Expect: 0.038

Matches (Bold Red): 27/188 fragment ions using 55 most intense peaks

\begin{tabular}{|c|c|c|c|c|c|c|c|c|c|c|c|c|c|c|}
\hline \# & b & $\mathrm{b}^{++}$ & b* & $b *^{++}$ & $b^{0}$ & $b^{0++}$ & Seq. & y & $y^{++}$ & y* & $y *^{++}$ & $y^{0}$ & $y^{0++}$ & $\#$ \\
\hline 1 & 132.0478 & 66.5275 & & & & & M & & & & & & & 18 \\
\hline 2 & 260.1063 & 130.5568 & 243.0798 & 122.0435 & & & $Q$ & 1801.0317 & 901.0195 & 178 & 892 & 0211 & 89 & 217 \\
\hline 3 & 347.1384 & 174.0728 & 0.1118 & 165.5595 & 329.1278 & 165.0675 & $\mathrm{~s}$ & 1672.9731 & 836.9902 & 1655.9465 & 828.4769 & 1654.9625 & 827.9849 & 916 \\
\hline 4 & 460.2224 & 230.6149 & 443.1959 & 222.1016 & 442.2119 & 221.6096 & $\mathrm{~L}$ & 1585.9411 & 793.4742 & 1568.9145 & 784.9609 & 1567.9305 & 784.4689 & 915 \\
\hline 5 & 561.2701 & 281.1387 & 544.2436 & 272.6254 & 543.2595 & 272.1334 & $\mathrm{~T}$ & 1472.8570 & 736.9321 & 1455.8305 & 728.4189 & 1454.8464 & 727.9269 & 914 \\
\hline 6 & 674.3542 & 337.6807 & 657.3276 & 329.1674 & 656.3436 & 328.6754 & $\mathrm{~L}$ & 1371.8093 & 686.4083 & 1354.7828 & 677.8950 & 1353.7988 & 677.4030 & 013 \\
\hline 7 & 789.3811 & 395.1942 & 772.3546 & 386.6809 & 771.3705 & 386.1889 & D & 1258.7253 & 629.8663 & 1241.6987 & 621.3530 & 1240.7147 & 620.8610 & 012 \\
\hline 8 & 888.4495 & 444.7284 & 871.4230 & 436.2151 & 870.4390 & 435.7231 & V & 1143.6983 & 572.3528 & 1126.6718 & 563.8395 & 1125.6878 & 563.3475 & 5 \\
\hline 9 & 1001.5336 & 501.2704 & 984.5070 & 492.7572 & 983.5230 & 492.2651 & $\mathrm{~L}$ & 1044.6299 & 522.8186 & 1027.6034 & 514.3053 & 1026.6193 & 513.8133 & 310 \\
\hline 10 & 1058.5550 & 529.7812 & 1041.5285 & 521.2679 & 1040.5445 & 520.7759 & G & 931.5459 & 466.2766 & 914.5193 & 457.7633 & 913.5353 & 457.2713 & 3 \\
\hline 11 & 1159.6027 & 580.3050 & 1142.5762 & 571.7917 & 1141.5922 & 571.2997 & $\mathrm{~T}$ & 874.5244 & 437.7658 & 857.4978 & 429.2526 & 856.5138 & 428.7606 & 8 \\
\hline 12 & 1246.6347 & 623.8210 & 1229.6082 & 615.3077 & 1228.6242 & 614.8157 & $\mathrm{~s}$ & 773.4767 & 387.2420 & 756.4502 & 378.7287 & 755.4661 & 378.2367 & $y$ \\
\hline 13 & 1375.6773 & 688.3423 & 1358.6508 & 679.8290 & 1357.6668 & 679.3370 & $E$ & 686.4447 & 343.7260 & 669.4181 & 335.2127 & 668.4341 & 334.7207 & 7 \\
\hline 14 & 1488.7614 & 744.8843 & 1471.7348 & 736.3711 & 1470.7508 & 735.8791 & $\mathrm{~L}$ & 557.4021 & 279.2047 & 540.3755 & 270.6914 & & & 5 \\
\hline 15 & 1601.8455 & 801.4264 & 1584.8189 & 792.9131 & 1583.8349 & 792.4211 & $\mathrm{~L}$ & 444.3180 & 222.6627 & 427.2915 & 214.1494 & & & 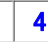 \\
\hline 16 & 1714.9295 & 857.9684 & 1697.9030 & 849.4551 & 1696.9190 & 848.9631 & L & 331.2340 & 166.1206 & 314.2074 & 157.6073 & & & 3 \\
\hline 17 & 1785.9666 & 893.4870 & 1768.9401 & 884.9737 & 1767.9561 & 884.4817 & A & 218.1499 & 109.5786 & 201.1234 & 101.0653 & & & 2 \\
\hline 18 & & & & & & & $\mathrm{~K}$ & 147.1128 & 74.0600 & 130.0863 & \begin{tabular}{|l|l|}
3 & 65.5468 \\
\end{tabular} & & & . \\
\hline
\end{tabular}
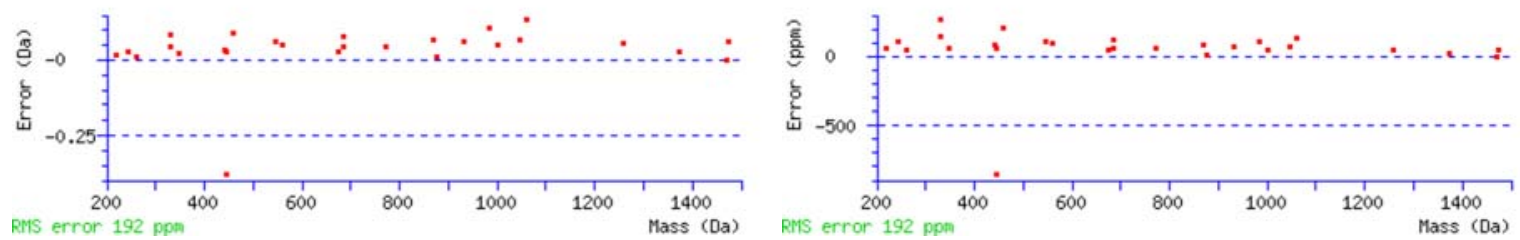


\section{Peptide View}

MS/MS Fragmentation of MSATFIGNSTAIQELFK

Found in gi|21746161|ref|NP_076205.1|, tubulin, beta [Mus musculus]

Match to Query 625: 1856.952648 from $\left(929.483600,2^{+}\right)$

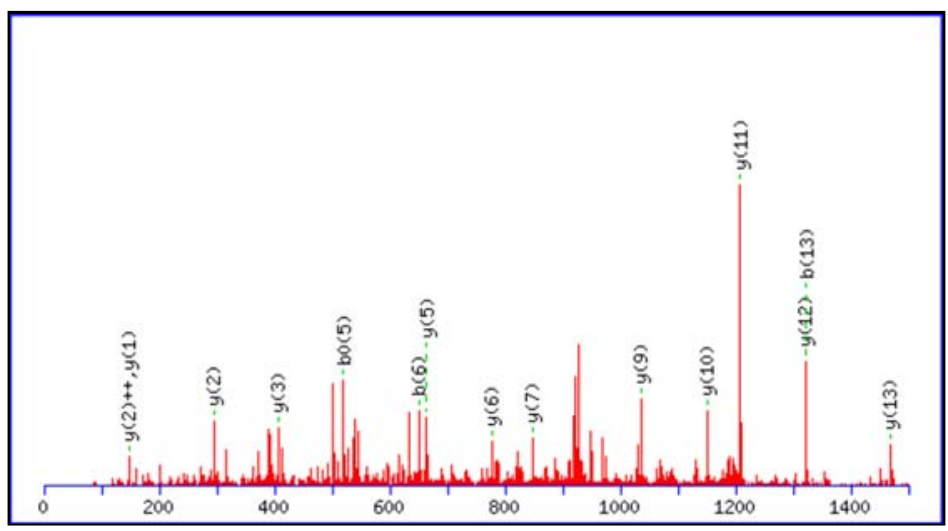

Monoisotopic mass of neutral peptide $\mathrm{Mr}$ (calc): 1856.9342

Ions Score: 77 Expect: 6. 2e-005

Matches (Bold Red): 15/170 fragment ions using 32 most intense peaks

\begin{tabular}{|c|c|c|c|c|c|c|c|c|c|c|c|c|c|c|}
\hline \# & b & $b^{++}$ & b* & $b *^{++}$ & $b^{0}$ & $b^{0++}$ & Seq. & $y$ & $y^{++}$ & y* & $y *^{++}$ & $y^{0}$ & $y^{0++}$ & $\#$ \\
\hline 1 & 132.0478 & 66.5275 & & & & & M & & & & & & & 17 \\
\hline 2 & 219.0798 & 110.0435 & & & 201.0692 & 101.0382 & $\mathrm{~s}$ & 1726.9010 & 863.9541 & 1709.8745 & 855.4409 & 1708.8904 & 854.9489 & 16 \\
\hline 3 & 290.1169 & 145.5621 & & & 272.1063 & 136.5568 & A & 1639.8690 & 820.4381 & 1622.8424 & 811.9248 & 1621.8584 & 811.4328 & 15 \\
\hline 4 & 391.1646 & 196.0859 & & & 373.1540 & 187.0806 & $T$ & 1568.8319 & 784.9196 & 1551.8053 & 776.4063 & 1550.8213 & 775.9143 & 14 \\
\hline 5 & 538.2330 & 269.6201 & & & 520.2224 & 260.6148 & $\mathrm{~F}$ & 1467.7842 & 734.3957 & 1450.7576 & 725.8825 & 1449.7736 & 725.3904 & 13 \\
\hline 6 & 651.3170 & 326.1622 & & & 633.3065 & 317.1569 & I & 1320.7158 & 660.8615 & 1303.6892 & 652.3482 & 1302.7052 & 651.8562 & 12 \\
\hline 7 & 708.3385 & 354.6729 & & & 690.3279 & 345.6676 & G & 1207.6317 & 604.3195 & 1190.6052 & 595.8062 & 1189.6211 & 595.3142 & 11 \\
\hline 8 & 822.3814 & 411.6944 & 805.3549 & 403.1811 & 804.3709 & 402.6891 & $\mathrm{~N}$ & 1150.6103 & 575.8088 & 1133.5837 & 567.2955 & 1132.5997 & 566.8035 & 10 \\
\hline 9 & 909.4135 & 455.2104 & 892.3869 & 446.6971 & 891.4029 & 446.2051 & $s$ & 1036.5673 & 518.7873 & 1019.5408 & 510.2740 & 1018.5568 & 509.7820 & 9 \\
\hline 10 & 1010.4611 & 505.7342 & 993.4346 & 497.2209 & 992.4506 & 496.7289 & $\mathrm{~T}$ & 949.5353 & 475.2713 & 932.5087 & 466.7580 & 931.5247 & 466.2660 & 8 \\
\hline 11 & 1081.4983 & 541.2528 & 1064.4717 & 532.7395 & 1063.4877 & 532.2475 & A & 848.4876 & 424.7474 & 831.4611 & 416.2342 & 830.4770 & 415.7422 & 7 \\
\hline 12 & 1194.5823 & 597.7948 & 1177.5558 & 589.2815 & 1176.5718 & 588.7895 & I & 777.4505 & 389.2289 & 760.4240 & 380.7156 & 759.4399 & 380.2236 & 6 \\
\hline 13 & 1322.6409 & 661.8241 & 1305.6143 & 653.3108 & 1304.6303 & 652.8188 & $Q$ & 664.3664 & 332.6869 & 647.3399 & 324.1736 & 646.3559 & 323.6816 & 5 \\
\hline 14 & 1451.6835 & 726.3454 & 1434.6569 & 717.8321 & 1433.6729 & 717.3401 & $E$ & 536.3079 & 268.6576 & 519.2813 & 260.1443 & 518.2973 & 259.6523 & 4 \\
\hline 15 & 1564.7675 & 782.8874 & 1547.7410 & 774.3741 & 1546.7570 & 773.8821 & L & 407.2653 & 204.1363 & 390.2387 & 195.6230 & & & 3 \\
\hline 16 & 1711.8360 & 856.4216 & 1694.8094 & 847.9083 & 1693.8254 & 847.4163 & $\mathrm{~F}$ & 294.1812 & 147.5942 & 277.1547 & 139.0810 & & & 2 \\
\hline 17 & & & & & & & $\mathrm{~K}$ & 147.1128 & 74.0600 & 130.0863 & 65.5468 & & & 1 \\
\hline
\end{tabular}

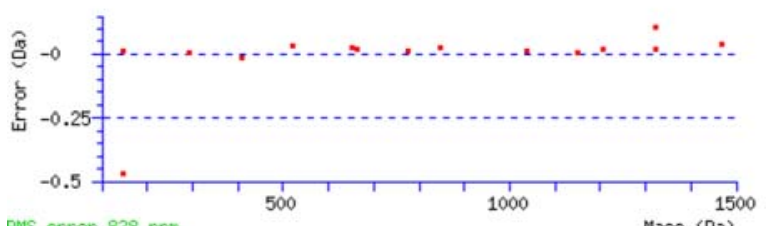

PMS error $828 \mathrm{ppm}$

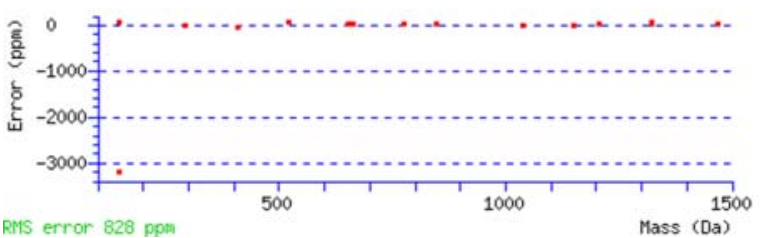




\section{Peptide View}

MS/MS Fragmentation of MTQIMFEAFNTPAMYVAIQAVLSLYASGR

Found in gi|30425250|ref|NP_780706.1|, hypothetical protein LOC238880 [Mus musculus]

Match to Query 898: 3253.778472 from(1085.600100,3+)

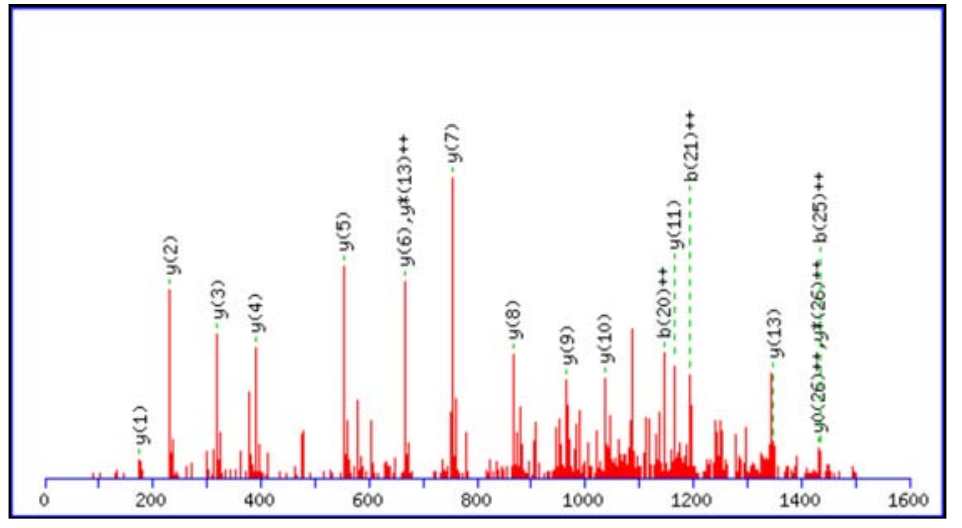

Monoisotopic mass of neutral peptide $\operatorname{Mr}$ (calc): 3254.5813

Variable modifications:

M1 : Oxidation (M)

M14 : 0xidation (M)

Ions Score: 62 Expect: 0.0065

Matches (Bold Red): 18/326 fragment ions using 27 most intense peaks

\begin{tabular}{|c|c|c|c|c|c|c|c|c|c|c|c|c|c|c|}
\hline \# & b & $b^{++}$ & $b *$ & $k^{++}$ & $b^{0}$ & ++ & & y & $y^{++}$ & $y *$ & + & $y^{0}$ & & \# \\
\hline 1 & 427 & 50 & & & & & & & & & & & & 29 \\
\hline 2 & 249.0904 & 488 & & & 798 & & & & & 3091.5267 & & 5427 & 1545.7750 & 28 \\
\hline 3 & 377.1489 & 9.0781 & 0.1224 & 80.5648 & 59.1384 & 180.0728 & $Q$ & 3007.5056 & 1504.2564 & 2990.4790 & 431 & 2989.4950 & 511 & 27 \\
\hline 4 & 490.2330 & 201 & 3.2064 & 069 & 472.2224 & 236.6149 & I & 2879.4470 & 144 & 62.4204 & 139 & 364 & 218 & 26 \\
\hline 5 & 621.2735 & 104 & 69 & 271 & 629 & 51 & M & 629 & 851 & 364 & 718 & 524 & 798 & 25 \\
\hline 6 & 768.3419 & 746 & 751. & 613 & 313 & 375.6693 & $F$ & 224 & 649 & 2959 & 516 & 2617.3119 & 596 & 2 \\
\hline 7 & 897.3845 & 449.1959 & 880.3579 & 440.6826 & 879.3739 & 440.1906 & $E$ & 2488.2540 & 1244.6307 & 2471.2275 & 1174 & 2470.2435 & 254 & 3 \\
\hline 8 & 968.4216 & 484.7144 & 51.3950 & 476.2012 & 950.4110 & 475.7091 & A & 2359.2114 & 094 & 2342.1849 & 1171.5961 & 2341.2009 & 041 & 22 \\
\hline 9 & 1115.4900 & 558.2486 & 1098.4634 & 549.7354 & 1097.4794 & 549.2434 & $F$ & 2288.1743 & 1144.5908 & 2271.1478 & 1136.0775 & 2270.1638 & .5855 & 21 \\
\hline 10 & 329 & 01 & 64 & 68 & 24 & 48 & $\mathrm{~N}$ & 2141.1059 & 566 & 4.0794 & 433 & 954 & 513 & 20 \\
\hline 11 & 1330.5806 & 665.7939 & 1313.5541 & 657.2807 & 1312.5700 & 656.7887 & $\mathrm{~T}$ & 2027.0630 & 351 & 2010.0364 & 1005.5219 & 2009.0524 & 1005.0299 & 19 \\
\hline 12 & 1427.6334 & 714.3203 & 1410.6068 & 705 & 1409.6228 & 705 & $P$ & .0153 & 113 & 1908.9888 & 954.9980 & 0047 & 60 & 18 \\
\hline 13 & 1498 & 89 & 39 & 56 & 99 & 74 & A & 26 & 49 & 00 & 16 & 0 & 96 & 7 \\
\hline 14 & 1645.7059 & 823.3566 & 1628.6793 & 814.8433 & 1627.6953 & 814.3513 & $M$ & 1757.9254 & 879.4664 & 1740.8989 & 870.9531 & 1739.9149 & 611 & 16 \\
\hline 15 & $1 \varepsilon$ & 2 & 427 & 0 & 1 & 0 & $\mathrm{Y}$ & 0 & 37 & 5 & 54 & 5 & 4 & 5 \\
\hline 16 & 1907.8376 & 954.4224 & 1890.8111 & 945.9092 & 1889.8270 & 945.4172 & V & 1447.8267 & 724.4170 & 1430 & .9037 & 161 & 17 & 14 \\
\hline 17 & 1978.8747 & 39.9410 & 1961.8482 & 981.4277 & 1960.8642 & 980.9357 & $A$ & 1348.7583 & 674.8828 & 1331.7318 & 666.3695 & 1330.7477 & 75 & . \\
\hline 18 & 2091.9588 & 30 & 2074.9322 & 1037 & 2073 & 77 & I & 12 & 42 & 46 & 10 & 66 & 90 & 12 \\
\hline 19 & 2220.0174 & 10.5123 & 2202.9908 & 1101.9990 & 2202.0068 & 1101.5070 & 8 & 1164.6371 & 582.8222 & 1147.6106 & 3089 & 266 & 69 & 1 \\
\hline 20 & 5 & 1 & 74.0279 & רי & 2 & 37.0256 & A & 36.5786 & 18.7929 & 1019.5520 & 510.2796 & 10 & 6 & 10 \\
\hline 21 & 2390.1229 & 1195.5651 & 2373.0963 & 1187.0518 & 2372.1123 & 1186.5598 & V & 965.5414 & 483.2744 & 948.5149 & 474.7611 & 947.5309 & 474.2691 & 9 \\
\hline 22 & 2503.2069 & 1252.1071 & 2486.1804 & 38 & 5.1964 & 1243.1018 & 1 & 30 & 433.7 & 65 & 425.2269 & 25 & 49 & 8 \\
\hline 23 & 2590.2390 & 1295.6231 & 2573.2124 & 1287.1099 & 2572.2284 & 1286.6178 & $S$ & 753.3890 & 377.1981 & 736.3624 & 368.6848 & 735.3784 & 368.1928 & 7 \\
\hline 24 & 2703.3230 & 1352.1652 & 2686.2965 & 1343.6519 & 2685.3125 & 1343.1599 & 1 & 569 & 821 & 304 & 688 & 464 & 768 & 6 \\
\hline 25 & 2866.3864 & 1433.6968 & 2849.3598 & 1425.1835 & 2848.3758 & 1424.6915 & $Y$ & 3.2729 & 1.1401 & $0 . \angle 403$ & 268.6268 & 535.2623 & 48 & 5 \\
\hline 26 & 2937.4235 & 1469.2154 & 2920.3969 & 1460.7021 & 2919.4129 & 1460.2101 & A & 390.2096 & 195.6084 & 373.1830 & 187.0951 & 372.1990 & 186.6031 & 4 \\
\hline 27 & 3024.4555 & 1512.7314 & 3007.4290 & 1504.2181 & 3006.4449 & 1503.7261 & $S$ & 724 & 160.0899 & 50.12 & 151.57 & 19 & 46 & 3 \\
\hline 28 & 3081.4770 & 1541.2421 & 3064.4504 & 1532.7288 & 3063.4664 & 1532.2368 & G & 232.1404 & 116.5738 & 215.1139 & 108.0606 & & & 2 \\
\hline 29 & & & & & & & $\mathrm{R}$ & 175.1190 & 38.0631 & 58.0924 & 79.5498 & & & 1 \\
\hline
\end{tabular}
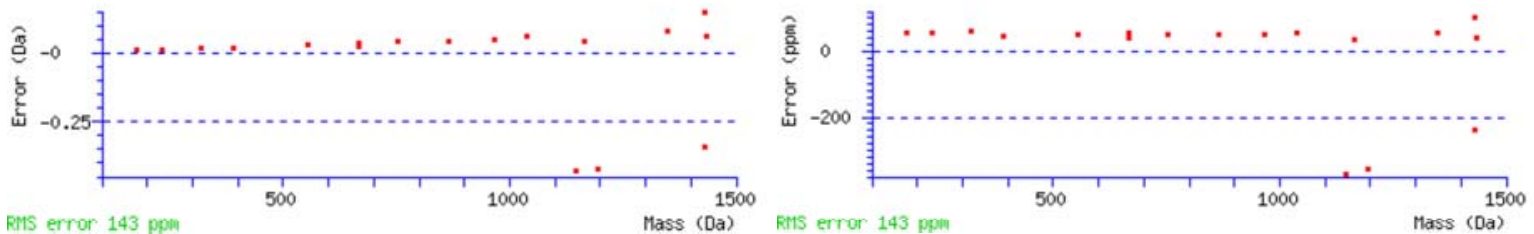


\section{Peptide View}

MS/MS Fragmentation of NIAGALAFWMANASELLNFIK

Found in gi|94402335|ref|XP_916103.2|, PREDICTED: similar to Afadin (Af-6 protein) isoform 4 [Mus musculus]

Match to Query 807: 2293.378048 from(1147.696300,2+)

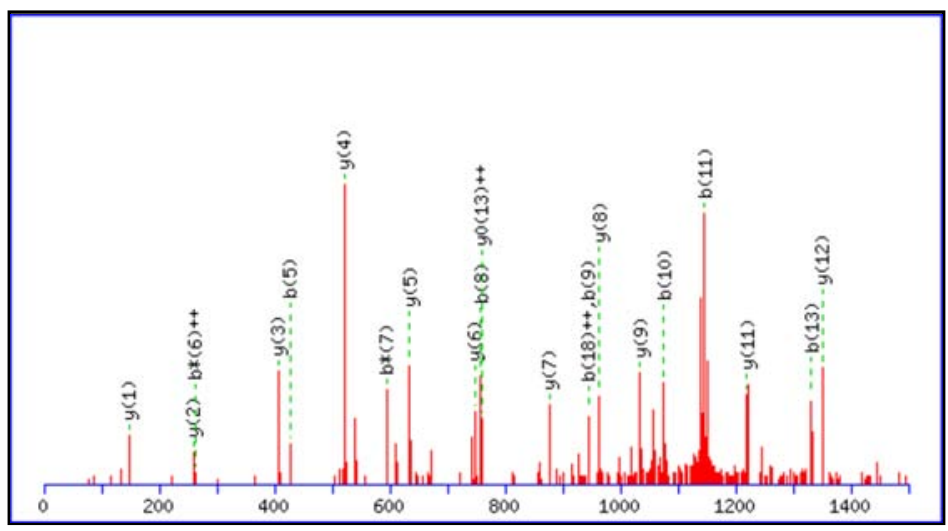

Monoisotopic mass of neutral peptide $\operatorname{Mr}(\mathrm{calc}): 2293.1928$

Ions Score: 76 Expect: 0.00013

Matches (Bold Red): 21/202 fragment ions using 36 most intense peaks

\begin{tabular}{|c|c|c|c|c|c|c|c|c|c|c|c|c|c|c|}
\hline \# & b & $b^{++}$ & b* & $\mathrm{b} *^{++}$ & $b^{0}$ & $b^{0++}$ & Seq. & y & $\mathrm{y}^{++}$ & y* & $y *^{++}$ & $y^{0}$ & $y^{0++}$ & \# \\
\hline 1 & 115.0502 & 58.0287 & 98.0237 & 49.5155 & & & $\mathrm{~N}$ & & & & & & & 21 \\
\hline 2 & 228.1343 & 114.5708 & 211.1077 & 106.0575 & & & I & 2180.1572 & 109 & 2163.1307 & 108 & 1466 & 108 & 20 \\
\hline 3 & 299.1714 & 0.0893 & 282.1448 & 61 & & & A & 67.0731 & 1034.0402 & 2050.0466 & 1025.5269 & 2049.0626 & 1025 & 19 \\
\hline 4 & 356.1928 & 178.6001 & 339.1663 & 170.0868 & & & G & 1996.0360 & 998.5217 & 1979.0095 & 990.0084 & 1978.0255 & 989.5164 & 18 \\
\hline 5 & 427.2299 & 214.1186 & 410.2034 & 205.6053 & & & A & 1939.0146 & 970.0109 & 1921.9880 & 961.4976 & 1921.0040 & 961.0056 & 17 \\
\hline 6 & 540.3140 & 270.6606 & 523.2875 & 262.1474 & & & L & 1867.9775 & 934.4924 & 1850.9509 & 925.9791 & 1849.9669 & 925.4871 & 16 \\
\hline 7 & 611.3511 & 306.1792 & 594.3246 & 297.6659 & & & A & 1754.8934 & 877.9503 & 1737.8669 & 869.4371 & 1736.8828 & 868.9451 & 15 \\
\hline 8 & 758.4195 & 379.7134 & 741.3930 & 371.2001 & & & $\mathrm{~F}$ & 1683.8563 & 842.4318 & 1666.8297 & 833.9185 & 1665.8457 & 833.4265 & 514 \\
\hline 9 & 944.4988 & 472.7531 & 927.4723 & 464.2398 & & & w & 1536.7879 & 768.8976 & 1519.7613 & 760.3843 & 1518.7773 & 759.8923 & 13 \\
\hline 10 & 1075.5393 & 538.2733 & 1058.5128 & 529.7600 & & & $M$ & 1350.7086 & 675.8579 & 1333.6820 & 667.3446 & 1332.6980 & 666 & 12 \\
\hline 11 & 1146.5764 & 573.7919 & 1129.5499 & 565.2786 & & & A & 1219.6681 & 610.3377 & 1202.6415 & 601.8244 & 1201.6575 & 601.3324 & 11 \\
\hline 12 & 1260.6194 & 630.8133 & 1243.5928 & 622.3000 & & & $\mathrm{~N}$ & 1148.6310 & 574.8191 & 1131.6044 & 566.3059 & 1130.6204 & 565.8138 & 10 \\
\hline 13 & 1331.6565 & 666.3319 & 1314.6299 & 657.8186 & & & A & 1034.5881 & 517.7977 & 1017.5615 & 509.2844 & 1016.5775 & 508.7924 & 9 \\
\hline 14 & 1418.6885 & 709.8479 & 1401.6619 & 701.3346 & 1400.6779 & 26 & $S$ & 963.5509 & 482.2791 & 946.5244 & 473.7658 & 945.5404 & 473.2738 & 8 \\
\hline 15 & 1547.7311 & 774.3692 & 1530.7045 & 765.8559 & 1529.7205 & 765.3639 & $E$ & 876.5189 & 438.7631 & 859.4924 & 430.2498 & 858.5083 & 429.7578 & 7 \\
\hline 16 & 1660.8151 & 830.9112 & 1643.7886 & 822.3979 & 1642.8046 & 821.9059 & L & 747.4763 & 374.2418 & 730.4498 & 365.7285 & & & 6 \\
\hline 17 & 1773.8992 & 887.4532 & \begin{tabular}{|l|}
1756.8727 \\
\end{tabular} & 878.9400 & 1755.8886 & 878.4480 & $L$ & 634.3923 & 317.6998 & 617.3657 & 309.1865 & & & 5 \\
\hline 18 & 1887.9421 & 944.4747 & 1870.9156 & 935.9614 & 1869.9316 & 935.4694 & $\mathrm{~N}$ & 521.3082 & 261.1577 & 504.2817 & 252.6445 & & & 4 \\
\hline 19 & 2035.0105 & 1018.0089 & 2017.9840 & 1009.4956 & 2017.0000 & 1009.0036 & $\mathrm{~F}$ & 407.2653 & 204.1363 & 390.2387 & 195.6230 & & & 0 \\
\hline 20 & 2148.0946 & 1074.5509 & 2131.0681 & 1066.0377 & 2130.0840 & 1065.5457 & I & 260.1969 & 130.6021 & 243.1703 & 122.0888 & & & 2 \\
\hline 21 & & & & & & & $\mathrm{~K}$ & 147.1128 & 74.0600 & 130.0863 & 65.5468 & & & \\
\hline
\end{tabular}
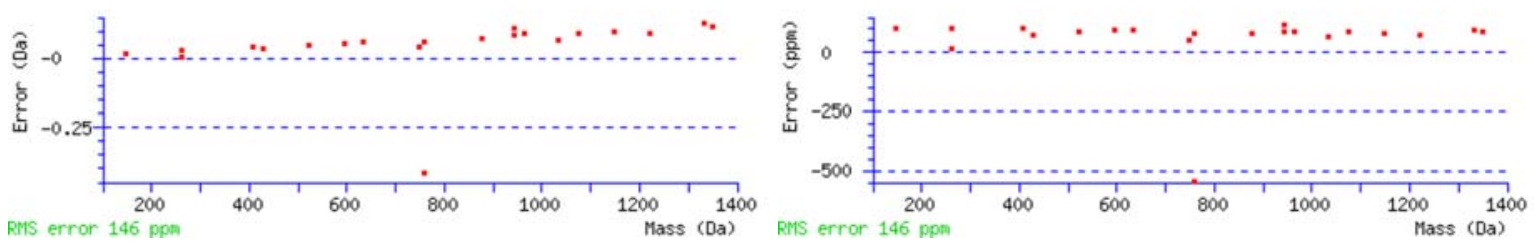


\section{Peptide View}

MS/MS Fragmentation of NIILEEGKEILVGDVGQTVDDPYTTFVK

Found in gi|6680924|ref|NP_031713.1|, cofilin 1, non-muscle [Mus musculus]

Match to Query 401: 3091.676172 from(1031.566000,3+)

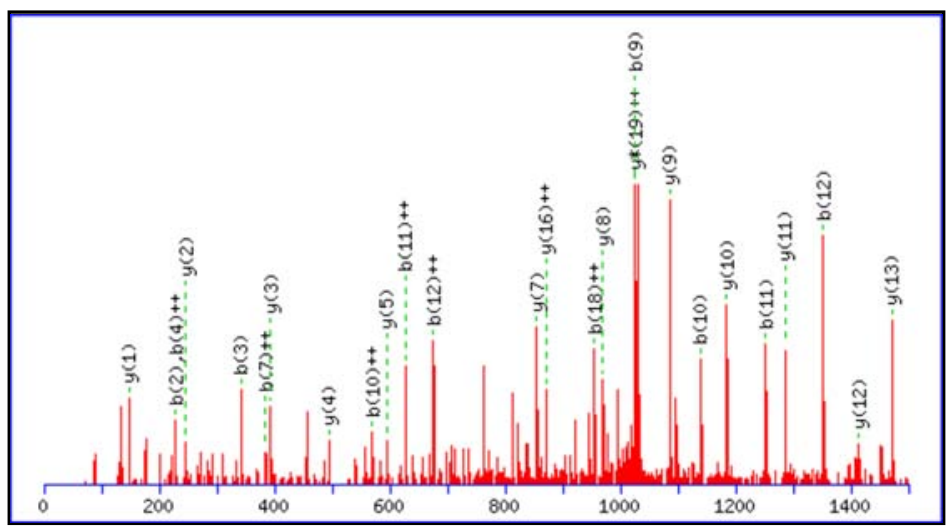

Monoisotopic mass of neutral peptide $\mathrm{Mr}$ (calc): 3091.5964

Ions Score: 61 Expect: 0.0078

Matches (Bold Red): 26/310 fragment ions using 47 most intense peaks

\begin{tabular}{|c|c|c|c|c|c|c|c|c|c|c|c|c|c|c|}
\hline \# & b & $b^{++}$ & b* & $b *^{++}$ & $b^{0}$ & $b^{0++}$ & Seq. & y & $y^{++}$ & y* & $y *^{++}$ & $y^{0}$ & $y^{0++}$ & $\#$ \\
\hline 7 & 115.0502 & 58.0287 & 98.0237 & 49.5155 & & & $\mathrm{~N}$ & & & & & & & 28 \\
\hline 2 & 228.1343 & 114.5708 & 211.1077 & 106.0575 & & & I & 2978.5608 & 840 & 2961.5342 & 1481.2708 & 2960.5502 & 1480.7788 & 27 \\
\hline 3 & 341.2183 & 171.1128 & 324.1918 & 162.5995 & & & I & 67 & 420 & 502 & 1424.7287 & 2847.4662 & 367 & 26 \\
\hline 4 & 454.3024 & 227.6548 & 437.2758 & 219.1416 & & & $L$ & 2752.3927 & 1376.7000 & 2735.3661 & 1368.1867 & 2734.3821 & 1367.6947 & 25 \\
\hline 5 & 583.3450 & 292.1761 & 566.3184 & 283.6629 & 565.3344 & 283.1708 & $\mathrm{E}$ & 2639.3086 & 1320.1579 & 2622.2821 & 1311.6447 & 2621.2980 & 1311.1527 & 24 \\
\hline 6 & 712.3876 & 356.6974 & 695.3610 & 348.1841 & 694.3770 & 347.6921 & $\mathrm{E}$ & 2510.2660 & 1255.6366 & 2493.2395 & 1247.1234 & 2492.2555 & 1246.6314 & 23 \\
\hline 7 & 769.4090 & 385.2082 & 752.3825 & 376.6949 & 751.3985 & 376.2029 & G & 34 & 1154 & 1969 & 1182.6021 & 2363.2129 & 118 & 22 \\
\hline 8 & 897.5040 & 449.2556 & 880.4774 & 440.7424 & 879.4934 & 440.2503 & $\mathrm{~K}$ & 2324.2020 & 1162.6046 & 2307.1754 & 1154.0913 & 2306.1914 & 1153.5993 & 21 \\
\hline 9 & 1026.5466 & 513.7769 & 1009.5200 & 505.2637 & 1008.5360 & 504.7716 & $E$ & 070 & 1098.5571 & 2179.0805 & 1090.0439 & 2178.0964 & 1089.5519 & 20 \\
\hline 10 & 1139.6306 & 570.3190 & 1122.6041 & 561.8057 & 1121.6201 & 561.3137 & 1 & 2067.0644 & 1034.0358 & 2050.0379 & 1025.5226 & 2049.0539 & 1025.0306 & 19 \\
\hline 11 & 1252.7147 & 626.8610 & 1235.6881 & 618.3477 & 1234.7041 & 617.8557 & $L$ & 1953.9804 & 977.4938 & 1936.9538 & 968.9805 & 1935.9698 & 968.4885 & 18 \\
\hline 2 & 1351.7831 & \begin{tabular}{|l|}
676.3952 \\
\end{tabular} & \begin{tabular}{|l|}
1334.7566 \\
\end{tabular} & 667.8819 & 1333.7725 & 667.3899 & V & 1840.8963 & 920.9518 & 1823.8698 & 912.4385 & 1822.8857 & 911.9465 & 17 \\
\hline 3 & 1408.8046 & 704.9059 & 1391.7780 & 696.3926 & 1390.7940 & 695.9006 & G & 1741.8279 & 871.4176 & 1724.8013 & 862.9043 & 1723.8173 & 862.4123 & 16 \\
\hline 4 & 1523.8315 & 762.4194 & 1506.8050 & 753.9061 & 1505.8209 & 753.4141 & D & 1684.8064 & 842.9069 & 1667.7799 & 834.3936 & 1666.7959 & 833.9016 & 15 \\
\hline 15 & 1622.8999 & 811.9536 & 1605.8734 & 803.4403 & 1604.8894 & 802.9483 & V & 1569.7795 & 785.3934 & 1552.7529 & 776.8801 & 1551.7689 & 776.3881 & 14 \\
\hline 6 & 679.9214 & 840.4643 & 1662.8948 & 831.9511 & 1661.9108 & 831.4590 & G & 1470.7111 & 735.8592 & 1453.6845 & 727.3459 & 1452.7005 & 726.8539 & 13 \\
\hline 17 & 1807.9800 & 904.4936 & 1790.9534 & 895.9803 & 1789.9694 & 895.4883 & $Q$ & 1413.6896 & 707.3484 & 1396.6631 & 698.8352 & 1395.6791 & 698.3432 & 12 \\
\hline 8 & \begin{tabular}{|l|}
1909.0276 \\
\end{tabular} & 955.0175 & 1892.0011 & 946.5042 & 1891.0171 & 946.0122 & $T$ & 1285.6310 & 643.3192 & 1268.6045 & 634.8059 & 1267.6205 & 634.3139 & 11 \\
\hline 19 & 2008.0960 & 1004.5517 & 1991.0695 & 996.0384 & 1990.0855 & 995.5464 & V & 1184.5834 & 592.7953 & 1167.5568 & 584.2820 & 1166.5728 & 583.7900 & 10 \\
\hline 20 & 2123.1230 & 1062.0651 & 2106.0964 & 1053.5519 & 2105.1124 & 1053.0598 & D & 1085.5150 & 543.2611 & 1068.4884 & 534.7478 & 1067.5044 & 534.2558 & 9 \\
\hline 21 & 2238.1499 & 1119.5786 & 2221.1234 & 1111.0653 & 2220.1394 & 1110.5733 & D & 970.4880 & 485.7476 & 953.4615 & 477.2344 & 952.4774 & 476.7424 & 8 \\
\hline 22 & \begin{tabular}{|l|}
2335.2027 \\
\end{tabular} & 1168.1050 & 2318.1761 & 1159.5917 & 2317.1921 & 1159.0997 & $P$ & 855.4611 & 428.2342 & 838.4345 & 419.7209 & 837.4505 & 419.2289 & 7 \\
\hline 23 & 2498.2660 & 1249.6366 & 2481.2395 & 1241.1234 & 2480.2555 & 1240.6314 & $Y$ & 758.4083 & 379.7078 & 741.3818 & 371.1945 & 740.3977 & 370.7025 & 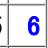 \\
\hline 24 & 2599.3137 & 1300.1605 & 2582.2871 & 1291.6472 & 2581.3031 & 1291.1552 & $T$ & 595.3450 & 298.1761 & 578.3184 & 289.6629 & 577.3344 & 289.1708 & 5 \\
\hline 25 & 2700.3614 & 1350.6843 & 2683.3348 & 1342.1711 & 2682.3508 & 1341.6790 & $T$ & 494.2973 & 247.6523 & 477.2708 & 239.1390 & 476.2867 & 238.6470 & 4 \\
\hline 26 & 2847.4298 & 1424.2185 & 2830.4032 & 1415.7053 & 2829.4192 & 1415.2132 & $\mathrm{~F}$ & 393.2496 & 197.1284 & 376.2231 & 188.6152 & & & 3 \\
\hline 27 & 2946.4982 & 1473.7527 & 2929.4716 & 1465.2395 & 2928.4876 & 1464.7475 & V & 246.1812 & 123.5942 & 229.1547 & 115.0810 & & & 2 \\
\hline 28 & & & & & & & $\mathrm{~K}$ & 147.1128 & 74.0600 & 130.0863 & 65.5468 & & & 1 \\
\hline
\end{tabular}

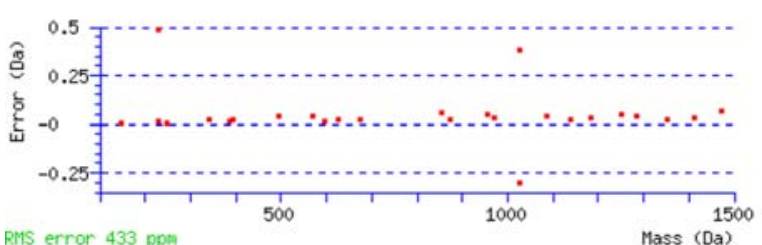

RMS error 433 ppm

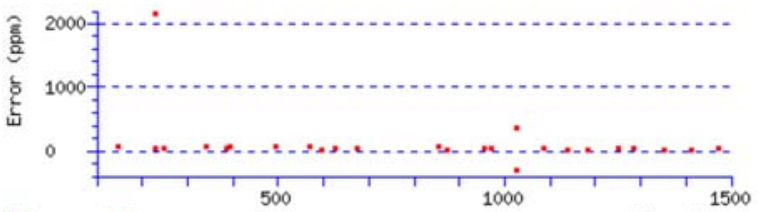

Mass (Da) 


\section{Peptide View}

MS/MS Fragmentation of NQVAMNPTNTVFDAK

Found in gi|31981690|ref|NP_112442.2|, heat shock protein 8 [Mus musculus]

Match to Query 378: 1648.860248 from $(825.437400,2+)$

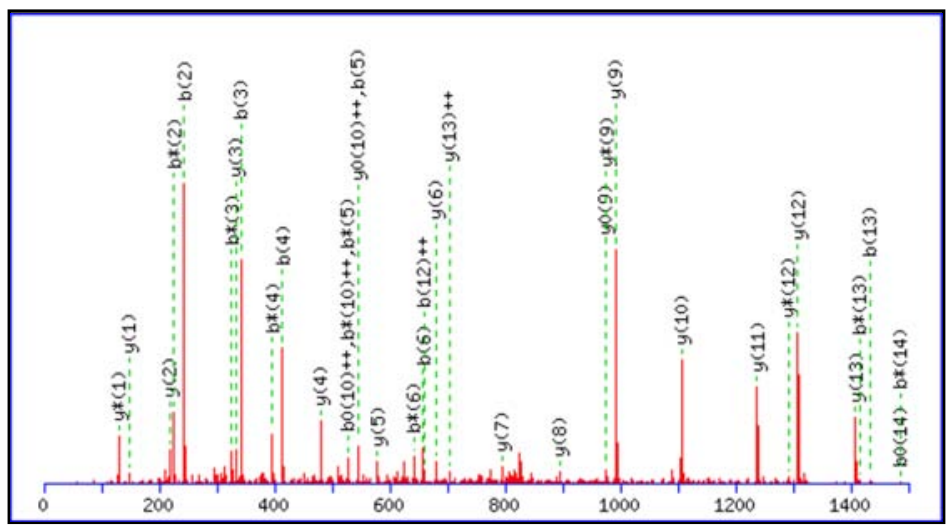

Monoisotopic mass of neutral peptide $\operatorname{Mr}$ (calc): 1648.7879

Ions Score: 86 Expect: $5.2 \mathrm{e}-006$

Matches (Bold Red): 36/150 fragment ions using 74 most intense peaks

\begin{tabular}{|c|c|c|c|c|c|c|c|c|c|c|c|c|c|c|}
\hline \# & b & $b^{++}$ & b* & $b *^{++}$ & $b^{0}$ & $b^{0++}$ & Seq. & y & $y^{++}$ & y* & $y *^{++}$ & $y^{0}$ & $y^{0++}$ & \# \\
\hline 1 & 115.0502 & 0287 & 98.0237 & 49.5155 & & & $\mathrm{~N}$ & & & & & & & 15 \\
\hline 2 & 243.1088 & 122.0580 & 226.0822 & 113.5448 & & & $Q$ & 1535.7522 & 768.3798 & 1518.7257 & 759.8665 & 1517.7417 & 759.3745 & 14 \\
\hline 3 & 2.1772 & 1.5922 & 325.1506 & 163.0790 & & & $v$ & 1407.6937 & 704.3505 & 1390.6671 & 3372 & 1389.6831 & 695.3452 & 13 \\
\hline 4 & 413.2143 & 207.1108 & 396.1878 & 198.5975 & & & A & 1308.6252 & 654.8163 & 1291.5987 & 646.3030 & 1290.6147 & 645.8110 & 12 \\
\hline 5 & 544.2548 & 2.6310 & 527.2282 & 264.1178 & & & M & 1237.5881 & \begin{tabular}{|l|}
619.2977 \\
\end{tabular} & 1220.5616 & 610.7844 & 1219.5776 & 610.2924 & 11 \\
\hline 6 & 658.2977 & 329.6525 & 641.2712 & 321.1392 & & & $\mathrm{~N}$ & 1106.5477 & 553.7775 & 1089.5211 & 545.2642 & 1088.5371 & 544.7722 & 10 \\
\hline 7 & 755.3505 & 378.1789 & 738.3239 & 369.6656 & & & $P$ & 992.5047 & 496.7560 & 975.4782 & 488.2427 & 974.4942 & \begin{tabular}{|l|}
487.7507 \\
\end{tabular} & 9 \\
\hline 8 & 856.3982 & 428.7027 & 839.3716 & 420.1894 & 838.3876 & 419.6974 & $\mathrm{~T}$ & 895.4520 & 448.2296 & 78.4254 & 439.7163 & 877.4414 & 439.2243 & 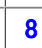 \\
\hline 9 & 970.4411 & \begin{tabular}{|l|}
485.7242 \\
\end{tabular} & 953.4145 & 477.2109 & 952.4305 & 476.7189 & $\mathrm{~N}$ & 794.4043 & 397.7058 & 777.3777 & 389.1925 & 776.3937 & 388.7005 & 7 \\
\hline 10 & \begin{tabular}{|l|}
1071.4888 \\
\end{tabular} & 536.2480 & 1054.4622 & 527.7347 & 1053.4782 & 527.2427 & $\mathrm{~T}$ & 680.3614 & 340.6843 & 663.3348 & 332.1710 & 662.3508 & 331.6790 & 6 \\
\hline 11 & 1170.5572 & 585.7822 & 1153.5306 & 577.2690 & 1152.5466 & 576.7769 & V & 579.3137 & 290.1605 & 562.2871 & 281.6472 & 561.3031 & 281.1552 & 5 \\
\hline 12 & 1317.6256 & 659.3164 & 1300.5990 & 650.8032 & 1299.6150 & 650.3111 & $\mathrm{~F}$ & 480.2453 & 240.6263 & 463.2187 & 232.1130 & 462.2347 & 231.6210 & 4 \\
\hline 131 & 1432.6525 & \begin{tabular}{|l|}
716.8299 \\
\end{tabular} & 1415.6260 & 708.3166 & \begin{tabular}{|l|}
1414.6420 \\
\end{tabular} & \begin{tabular}{|l|}
707.8246 \\
\end{tabular} & D & 333.1769 & \begin{tabular}{|l|}
167.0921 \\
\end{tabular} & 316.1503 & 158.5788 & 315.1663 & 158.0868 & 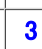 \\
\hline 14 & 1503.6896 & 752.3485 & 1486.6631 & 743.8352 & 1485.6791 & 743.3432 & A & 218.1499 & 109.5786 & & 101.0653 & & & 2 \\
\hline 15 & & & & & & & $\mathrm{~K}$ & 147.1128 & 74.0600 & 130.0863 & 65.5468 & & & 1 \\
\hline
\end{tabular}
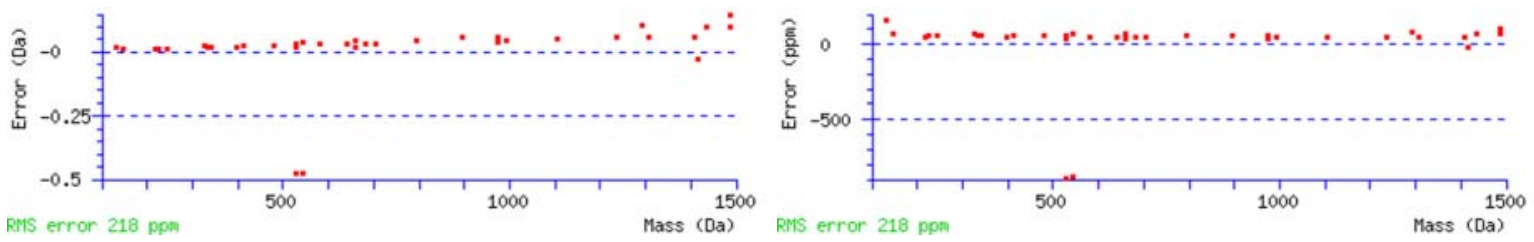


\section{Peptide View}

MS/MS Fragmentation of QAGGGAGPPNPSLNGSAPR

Found in gi|63723564|ref|XP_484699.2|, PREDICTED: similar to src homology 2 domain-containing transforming protein C [Mus musculus]

Match to Query 633: 1703.928048 from( $(852.971300,2+)$

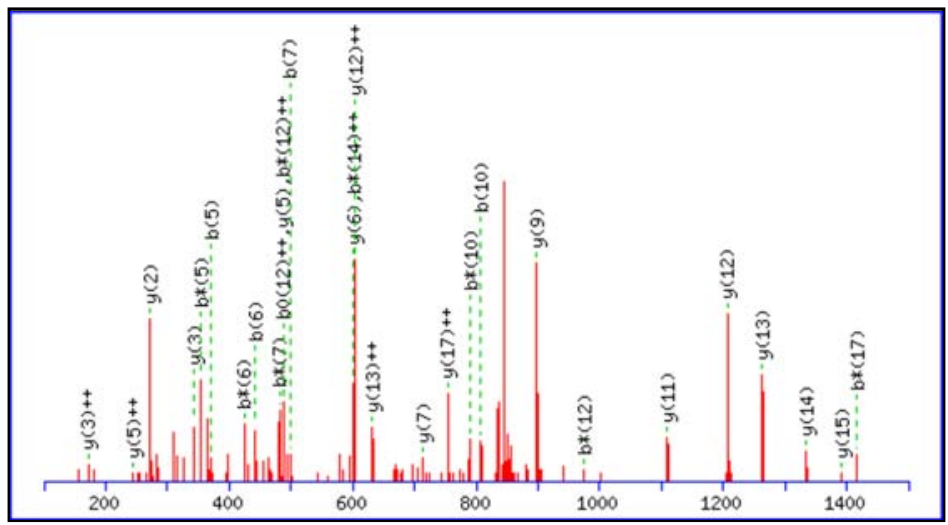

Monoisotopic mass of neutral peptide $\operatorname{Mr}(\mathrm{calc}): 1703.8339$

Ions Score: 56 Expect: 0.006

Matches (Bold Red): 29/188 fragment ions using 68 most intense peaks

\begin{tabular}{|c|c|c|c|c|c|c|c|c|c|c|c|c|c|c|}
\hline \# & b & $b^{++}$ & b* & $\mathrm{b} *^{++}$ & $b^{0}$ & $b^{0++}$ & Seq. & $y$ & $y^{++}$ & y* & $y *^{++}$ & $y^{0}$ & $y^{0++}$ & \# \\
\hline 1 & 129.0659 & 65.0366 & 112.0393 & 56.5233 & & & $Q$ & & & & & & & 19 \\
\hline 2 & 200.1030 & 100.5551 & 183.0764 & 92.0418 & & & A & 1576.7826 & 788.8949 & 1559.7561 & 780.3817 & 1558.7720 & 779.8897 & 18 \\
\hline 3 & 257.1244 & 129.0659 & 240.0979 & 120.5526 & & & $G$ & 1505.7455 & 753.3764 & 1488.7189 & 744.8631 & 1487.7349 & 744.3711 & 17 \\
\hline 4 & 314.1459 & 157.5766 & 297.1193 & 149.0633 & & & G & 1448.7240 & 724.8657 & 1431.6975 & 716.3524 & 1430.7135 & 715.8604 & 16 \\
\hline 5 & 371.1673 & 186.0873 & 354.1408 & 177.5740 & & & G & 1391.7026 & 696.3549 & 1374.6760 & 687.8416 & 1373.6920 & 687.3496 & 15 \\
\hline 6 & 442.2045 & 221.6059 & 425.1779 & 213.0926 & & & A & 1334.6811 & 667.8442 & 1317.6546 & 659.3309 & 1316.6705 & 658.8389 & 14 \\
\hline 7 & 499.2259 & 250.1166 & 482.1994 & 241.6033 & & & G & 1263.6440 & 632.3256 & 1246.6175 & 623.8124 & 1245.6334 & 623.3204 & 13 \\
\hline 8 & 596.2787 & 298.6430 & 579.2521 & 290.1297 & & & $\mathrm{P}$ & 1206.6225 & 603.8149 & 1189.5960 & 595.3016 & 1188.6120 & 594.8096 & 12 \\
\hline 9 & 693.3314 & 347.1694 & 676.3049 & 338.6561 & & & $P$ & 1109.5698 & 555.2885 & 1092.5432 & 546.7753 & 1091.5592 & 546.2832 & 11 \\
\hline 10 & 807.3744 & 404.1908 & 790.3478 & 395.6775 & & & $\mathrm{~N}$ & 1012.5170 & 506.7621 & 995.4905 & 498.2489 & 994.5065 & 497.7569 & 10 \\
\hline 11 & 904.4271 & 452.7172 & 887.4006 & 444.2039 & & & $P$ & 898.4741 & 449.7407 & 881.4475 & 441.2274 & 880.4635 & 440.7354 & 9 \\
\hline 12 & 991.4592 & 496.2332 & 974.4326 & 487.7199 & 973.4486 & 487.2279 & s & 801.4213 & 401.2143 & 784.3948 & 392.7010 & 783.4108 & 392.2090 & 8 \\
\hline 13 & 1104.5432 & 552.7752 & 1087.5167 & 544.2620 & 1086.5327 & 543.7700 & L & 714.3893 & 357.6983 & 697.3628 & 349.1850 & 696.3787 & 348.6930 & 7 \\
\hline 14 & 1218.5861 & 609.7967 & 1201.5596 & 601.2834 & 1200.5756 & 600.7914 & $\mathrm{~N}$ & 601.3052 & 301.1563 & 584.2787 & 292.6430 & 583.2947 & 292.1510 & 6 \\
\hline 15 & 1275.6076 & 638.3074 & 1258.5811 & \begin{tabular}{|l|}
629.7942 \\
\end{tabular} & 1257.5970 & 629.3022 & G & 487.2623 & 244.1348 & 470.2358 & 235.6215 & 469.2517 & 235.1295 & 5 \\
\hline 16 & 1362.6396 & 681.8235 & 1345.6131 & 673.3102 & 1344.6291 & 672.8182 & $\mathrm{~s}$ & 430.2409 & 215.6241 & 413.2143 & 207.1108 & 412.2303 & 206.6188 & 4 \\
\hline 17 & 1433.6767 & 717.3420 & 1416.6502 & \begin{tabular}{|l|}
708.8287 \\
\end{tabular} & 1415.6662 & 708.3367 & A & 343.2088 & 172.1080 & 326.1823 & 163.5948 & & & 3 \\
\hline 18 & 1530.7295 & 765.8684 & 1513.7030 & 757.3551 & 1512.7189 & 756.8631 & $P$ & 272.1717 & 136.5895 & 255.1452 & 128.0762 & & & 2 \\
\hline 19 & & & & & & & $R$ & 175.1190 & 88.0631 & 158.0924 & 79.5498 & & & 1 \\
\hline
\end{tabular}

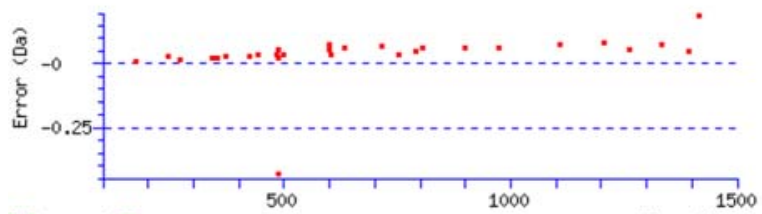

RMS error 182 ppm

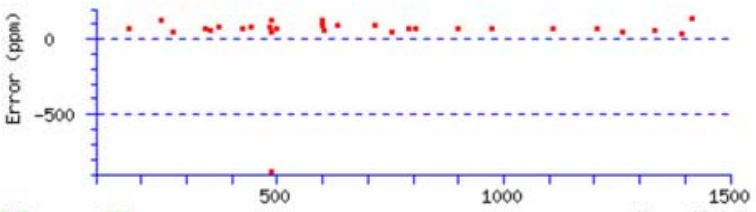

Mass (Da) 


\section{Peptide View}

MS/MS Fragmentation of QPVYIIMELVPGGDFLTFLR

Found in gi|6679773|ref|NP_032026.1|, fer (fms/fps related) protein kinase, testis specific 2 isoform b [Mus musculus]

Match to Query 740: 2313.282648 from(1157.648600,2+)

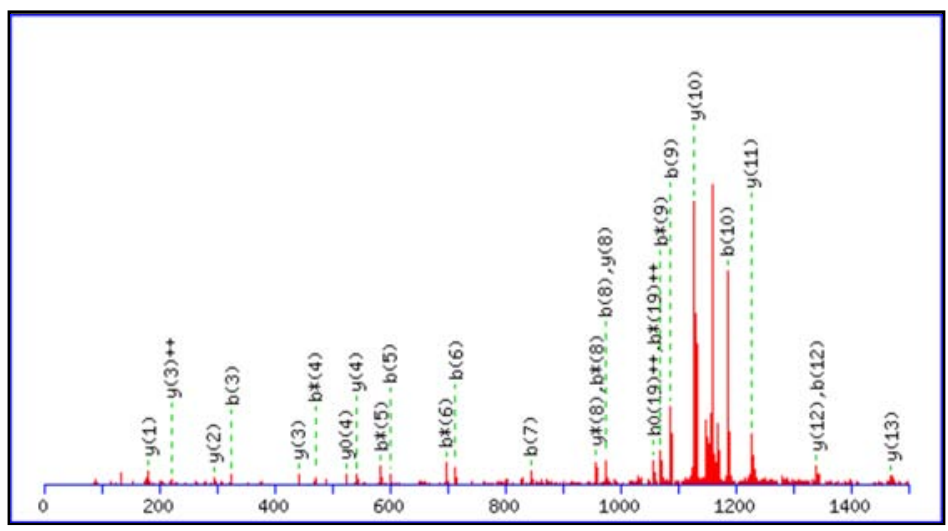

Monoisotopic mass of neutral peptide $\operatorname{Mr}(\mathrm{calc}): 2313.2538$

Variable modifications:

R20 : Arginine-13C6 (R-13C6)

Ions Score: 53 Expect: 0.033

Matches (Bold Red): 27/208 fragment ions using 45 most intense peaks

\begin{tabular}{|c|c|c|c|c|c|c|c|c|c|c|c|c|c|c|}
\hline \# & b & $\mathrm{b}^{++}$ & b* & $\mathrm{b} *^{++}$ & $b^{0}$ & $0++$ & Seq & $y$ & ++ & y* & +++ & $y^{0}$ & $0++$ & \# \\
\hline 1 & 129.0659 & .0366 & 112.0393 & 56.5233 & & & Q & & & & & & & 20 \\
\hline 2 & 226.1186 & 3.5629 & 209.0921 & 105.0497 & & & $P$ & & & 9 & & & & 19 \\
\hline 3 & 325.1870 & 3.0972 & 3 & 1 & & & V & & & 2072.1232 & & 2071.1392 & .0732 & 18 \\
\hline 4 & 488.2504 & 4.6288 & 471.2 & 236.1155 & & & $Y$ & 90.0813 & 43 & 1973.0548 & & 1972.0707 & 5390 & 17 \\
\hline 5 & 601.3344 & 1.1708 & 079 & 292.6576 & & & & 1827.0180 & 4.0126 & 1809.9914 & 4994 & 1809.0074 & 0073 & 16 \\
\hline 6 & 714.4185 & 57.7129 & 697. & 349.1996 & & & I & 1713 & 857.4 & 1696.9074 & & 1695.9234 & 653 & 15 \\
\hline 7 & 845.4590 & 3.2331 & 828.4324 & 414.7198 & & & M & 1600.8499 & 800.9286 & 1583.8233 & 4153 & 1582.8393 & 9233 & 14 \\
\hline 8 & 974.5015 & 37.7544 & 957.4 & 479.2411 & 0.4910 & & E & 1469 & & 7828 & & 1451.7988 & 30 & 1 \\
\hline 9 & 1087.5856 & 544.2964 & 1070.5591 & 535.7832 & 1069.5750 & 535.2912 & L & 1340.7668 & 670.8870 & 1323.7402 & .3738 & 1322.7562 & 8817 & 12 \\
\hline 10 & 1186.6540 & 3.8306 & 15 & 74 & & & V & 1227 & & 12 & 317 & 120 & 997 & 11 \\
\hline 11 & 1283.7068 & 642.3570 & 1266.6802 & 633.8438 & 1265.6962 & 17 & $P$ & 1128.6143 & 564.8108 & 1111.5878 & 2975 & 1110.6038 & 555.8055 & 10 \\
\hline 12 & 1340.7282 & 0.8678 & 1323 & 662.3545 & & & 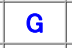 & 1031.5616 & 516.2844 & 1014.5350 & 507.7711 & 1013.5510 & 507.2791 & 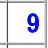 \\
\hline 13 & 1397.7497 & 699.3785 & 1380.7231 & 690.8652 & 1379.7391 & 690 & G & 974.5401 & \begin{tabular}{|l|}
487.7737 \\
\end{tabular} & 957.5135 & 479.2604 & 956.5295 & 478.7684 & 8 \\
\hline 14 & 1512.7766 & 756.8920 & 1495.7501 & 748.3787 & 1494.7661 & & D & 917.5186 & 459.2630 & 900.4921 & 450.7497 & 899.5081 & 450.2577 & 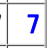 \\
\hline 15 & 1659.8450 & 830.4262 & 1642.8185 & 821.9129 & 1641.8345 & 821.4209 & $\mathrm{~F}$ & 802.4917 & 401.7495 & 785.4651 & 393.2362 & 784.4811 & 392.7442 & 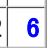 \\
\hline 16 & 1772.9291 & 886.9682 & 1755.9026 & 878.4549 & 1754.9185 & 877.9629 & L & 655.4233 & 328.2153 & 638.3967 & 319.7020 & 637.4127 & 319.2100 & 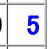 \\
\hline 17 & \begin{tabular}{|l|}
1873.9768 \\
\end{tabular} & 937.4920 & 1856.9502 & 928.9788 & 1855.9662 & 928.4867 & $\mathrm{~T}$ & 542.3392 & 271.6733 & 525.3127 & 263.1600 & 524.3287 & 262.6680 & 4 \\
\hline 18 & 2021.0452 & 1011 & 2004.0186 & 1002.5130 & 2003.0346 & 1002.0210 & $\mathrm{~F}$ & 441.2915 & 221.1494 & 424.2650 & 212.6361 & & & 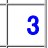 \\
\hline 19 & 2134.1293 & 1067.5683 & 2117.1027 & 1059.0550 & 2116.1187 & 1058.5630 & L & 294.2231 & 147.6152 & 277.1966 & 139.1019 & & & 2 \\
\hline 0 & & & & & & & $\mathrm{R}$ & 181.1391 & 91.0732 & 164.1125 & 82.5599 & & & \\
\hline
\end{tabular}
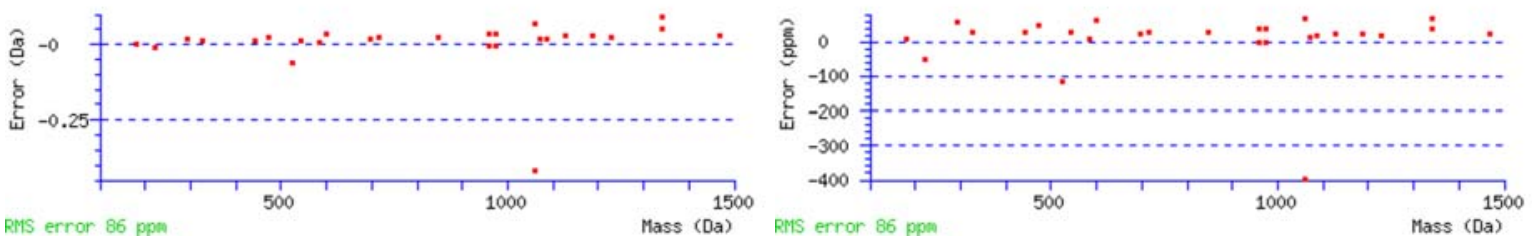


\section{Peptide View}

MS/MS Fragmentation of SDVWSFGILLTELTTK

Found in gi|70794809|ref|NP_001020566.1|, Rous sarcoma oncogene isoform 2 [Mus musculus]

Match to Query 678: 1809.103448 from $(905.559000,2+)$

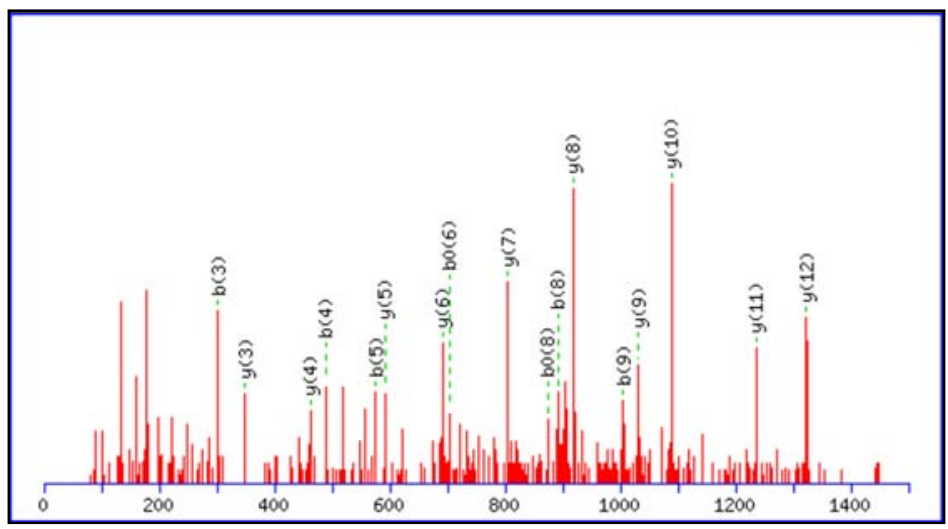

Monoisotopic mass of neutral peptide $\mathrm{Mr}$ (calc): 1808.9560

Ions Score: 62 Expect: 0.0026

Matches (Bold Red): 17/148 fragment ions using 35 most intense peaks

\begin{tabular}{|c|c|c|c|c|c|c|c|c|c|c|c|c|}
\hline \# & b & $\mathrm{b}^{++}$ & $b^{0}$ & $b^{0++}$ & Seq. & $\mathrm{y}$ & $y^{++}$ & y* & $y *^{++}$ & $y^{0}$ & $y^{0++}$ & $\#$ \\
\hline 1 & 88.0393 & 44.5233 & 70.0287 & 35.5180 & s & & & & & & & 16 \\
\hline 2 & 203.0662 & 102.0368 & 185.0557 & 93.0315 & D & 1722.9312 & 861.9693 & 1705.9047 & 853.4560 & 1704 & 852.9640 & 15 \\
\hline 3 & 302.1347 & 151.5710 & 284.1241 & 142.5657 & V & 1607.9043 & 804.4558 & 1590.8777 & 795.9425 & 1589.8937 & 795.4505 & 14 \\
\hline 4 & 488.2140 & 244.6106 & 470.2034 & 235.6053 & W & 1508.8359 & 754.9216 & 1491.8093 & 746.4083 & 1490.8253 & 745.9163 & 13 \\
\hline 5 & 575.2460 & 288.1266 & 557.2354 & 279.1214 & $\mathrm{~s}$ & 1322.7566 & 661.8819 & 1305.7300 & 653.3686 & 1304.7460 & 652.8766 & 12 \\
\hline 6 & 722.3144 & 361.6608 & 704.3038 & 352.6556 & $\mathrm{~F}$ & 1235.7245 & 618.3659 & 1218.6980 & 609.8526 & 1217.7140 & 609.3606 & 1 \\
\hline 7 & 779.3359 & 390.1716 & 761.3253 & 381.1663 & G & 1088.6561 & 544.8317 & 1071.6296 & 536.3184 & 1070.6456 & 535.8264 & 10 \\
\hline 8 & 892.4199 & 446.7136 & 874.4094 & 437.7083 & I & 1031.6347 & 516.3210 & 1014.6081 & 507.8077 & 1013.6241 & 507.3157 & - \\
\hline 9 & 1005.5040 & 503.2556 & 987.4934 & 494.2503 & L & 918.5506 & 459.7789 & 901.5241 & 451.2657 & 900.5400 & 450.7737 & 8 \\
\hline 10 & 1118.5880 & 559.7977 & 1100.5775 & 550.7924 & L & 805.4666 & 403.2369 & 788.4400 & 394.7236 & 787.4560 & 394.2316 & 7 \\
\hline 11 & 1219.6357 & 610.3215 & 1201.6252 & 601.3162 & $\mathrm{~T}$ & 692.3825 & 346.6949 & 675.3559 & 338.1816 & 674.3719 & 337.6896 & 6 \\
\hline 12 & 1348.6783 & 674.8428 & 1330.6678 & 665.8375 & $\mathrm{E}$ & 591.3348 & 296.1710 & 574.3083 & 287.6578 & 573.3242 & 287.1658 & 5 \\
\hline 13 & 1461.7624 & 731.3848 & 1443.7518 & 722.3795 & L & 462.2922 & 231.6497 & 445.2657 & 223.1365 & 444.2817 & 222.6445 & 4 \\
\hline 14 & 1562.8101 & 781.9087 & 1544.7995 & 772.9034 & $\mathrm{~T}$ & 349.2082 & 175.1077 & 332.1816 & 166.5944 & 331.1976 & 166.1024 & 3 \\
\hline 15 & 1663.8577 & 832.4325 & 1645.8472 & 823.4272 & $T$ & 248.1605 & 124.5839 & 231.1339 & 116.0706 & 230.1499 & 115.5786 & 2 \\
\hline 16 & & & & & $\mathrm{~K}$ & 147.1128 & 74.0600 & 130.0863 & 65.5468 & & & 1 \\
\hline
\end{tabular}
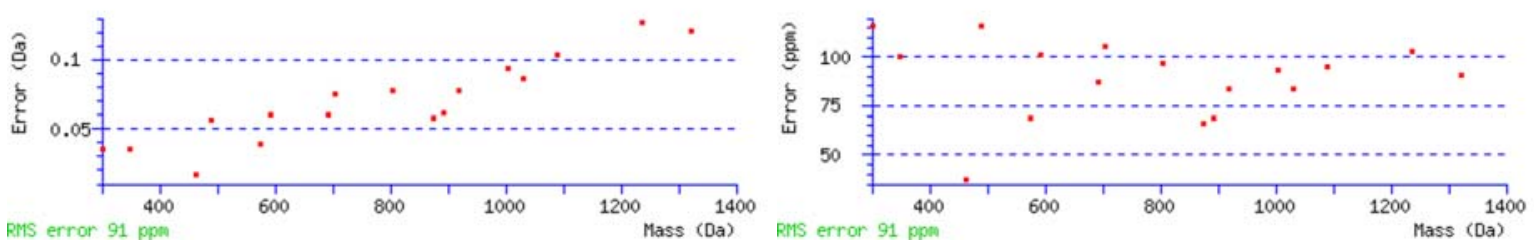


\section{Peptide View}

MS/MS Fragmentation of SDVWSFGILLTELVTK

Found in gi|6679879|ref|NP_032080.1|, protein-tyrosine kinase fyn [Mus musculus]

Match to Query 676: 1807.136848 from(904.575700,2+)

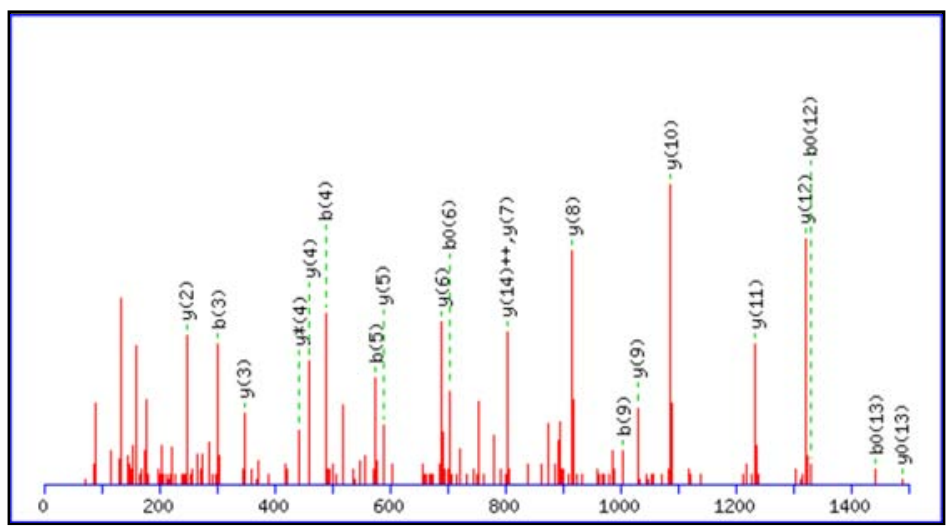

Monoisotopic mass of neutral peptide $\operatorname{Mr}$ (calc): 1806.9767

Ions Score: 84 Expect: $1.4 \mathrm{e}-005$

Matches (Bold Red) : 21/148 fragment ions using 32 most intense peaks

\begin{tabular}{|c|c|c|c|c|c|c|c|c|c|c|c|c|}
\hline \# & b & $\mathrm{b}^{++}$ & $b^{0}$ & $b^{0++}$ & Seq. & y & $y^{++}$ & y* & $y *^{++}$ & $y^{0}$ & $y^{0++}$ & $\#$ \\
\hline 1 & 88.0393 & 44.5233 & 70.0287 & 35.5180 & $\mathrm{~s}$ & & & & & & & 16 \\
\hline 2 & 203.0662 & 102.0368 & 185.0557 & 93.0315 & D & 1720.9520 & 860.9796 & 1703.9254 & 852.4663 & 1702.9414 & 851.9743 & 15 \\
\hline 3 & 302.1347 & 151.5710 & 284.1241 & 142.5657 & V & 1605.9250 & 803.4661 & 1588.8985 & 794.9529 & 1587.9145 & 794.4609 & 14 \\
\hline 4 & 488.2140 & 244.6106 & 470.2034 & 235.6053 & W & 1506.8566 & 753.9319 & 1489.8301 & 745.4187 & 1488.8460 & 744.9267 & 13 \\
\hline 5 & 575.2460 & 288.1266 & 557.2354 & 279.1214 & $\mathrm{~s}$ & 1320.7773 & 660.8923 & 1303.7508 & 652.3790 & 1302.7667 & 651.8870 & 12 \\
\hline 6 & 722.3144 & 361.6608 & 704.3038 & 352.6556 & $\mathrm{~F}$ & 1233.7453 & 617.3763 & 1216.7187 & 608.8630 & 1215.7347 & 608.3710 & 11 \\
\hline 7 & 779.3359 & 390.1716 & 761.3253 & 381.1663 & G & 1086.6769 & 543.8421 & 1069.6503 & 535.3288 & 1068.6663 & 534.8368 & 10 \\
\hline 8 & 892.4199 & 446.7136 & 874.4094 & 437.7083 & I & 1029.6554 & 515.3313 & 1012.6289 & 506.8181 & 1011.6448 & 506.3261 & 9 \\
\hline 9 & 1005.5040 & 503.2556 & 987.4934 & 494.2503 & L & 916.5713 & 458.7893 & 899.5448 & $450.2760 \mid$ & 898.5608 & 449.7840 & 8 \\
\hline 10 & 1118.5880 & 559.7977 & 1100.5775 & 550.7924 & L & 803.4873 & 402.2473 & 786.4607 & 393.7340 & 785.4767 & 393.2420 & 7 \\
\hline 11 & 1219.6357 & 610.3215 & 1201.6252 & 601.3162 & $\mathrm{~T}$ & 690.4032 & 345.7052 & 673.3767 & 337.1920 & 672.3927 & 336.7000 & 6 \\
\hline 12 & 1348.6783 & 674.8428 & 1330.6678 & 665.8375 & $\mathrm{E}$ & 589.3555 & 295.1814 & 572.3290 & 286.6681 & 571.3450 & 286.1761 & 5 \\
\hline 13 & 1461.7624 & 731.3848 & 1443.7518 & 722.3795 & L & 460.3130 & 230.6601 & 443.2864 & 222.1468 & 442.3024 & 221.6548 & 4 \\
\hline 14 & 1560.8308 & 780.9190 & 1542.8202 & 771.9137 & V & 347.2289 & 174.1181 & 330.2023 & 165.6048 & 329.2183 & 165.1128 & 3 \\
\hline 15 & 1661.8785 & 831.4429 & 1643.8679 & 822.4376 & $T$ & 248.1605 & 124.5839 & 231.1339 & 116.0706 & 230.1499 & 115.5786 & 2 \\
\hline 16 & & & & & $K$ & 147.1128 & 74.0600 & 130.0863 & 65.5468 & & & 1 \\
\hline
\end{tabular}
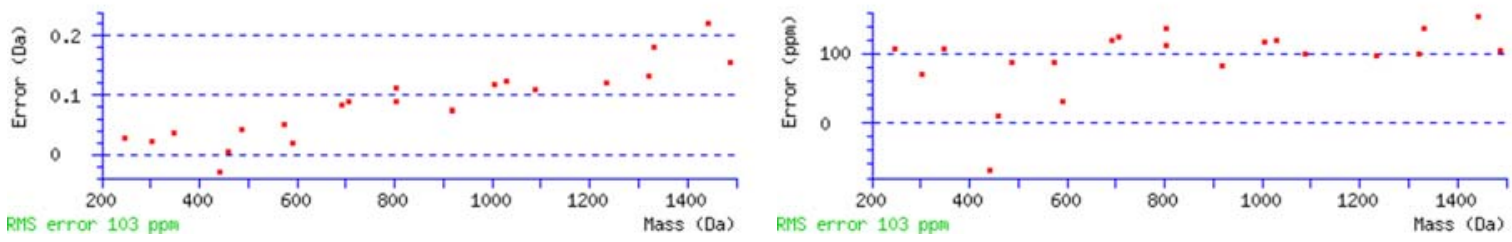


\section{Peptide View}

MS/MS Fragmentation of SSSLEGFHSQYK

Found in gi|31542871|ref|NP_067331.2|, growth factor receptor bound protein 2-associated protein 1 [Mus musculus]

Match to Query 388: 1368.650448 from(685.332500,2+)

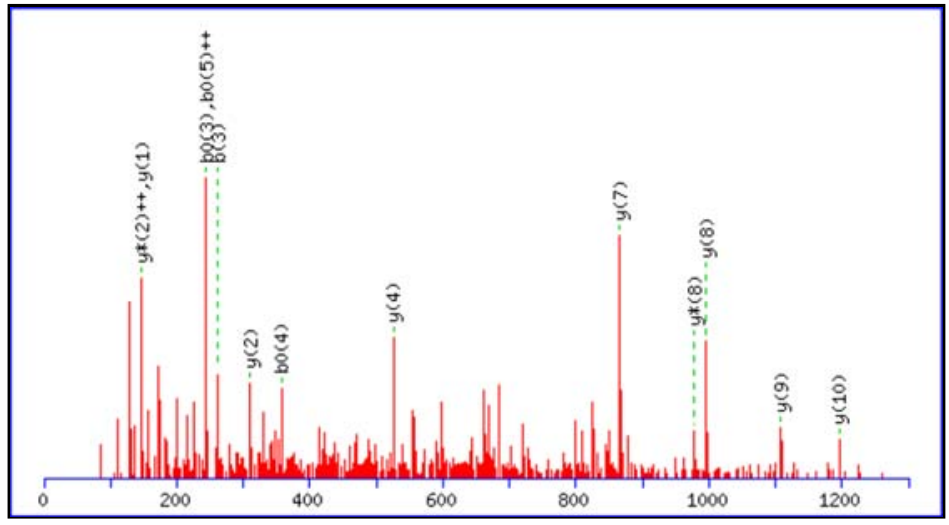

Monoisotopic mass of neutral peptide $\operatorname{Mr}(\mathrm{calc}): 1368.6310$

Ions Score: 51 Expect: 0.012

Matches (Bold Red): 13/108 fragment ions using 20 most intense peaks

\begin{tabular}{|c|c|c|c|c|c|c|c|c|c|c|c|c|c|c|}
\hline$\#$ & b & $b^{++}$ & $b *$ & $b *^{++}$ & $b^{0}$ & $b^{0++}$ & Seq. & $y$ & $y^{++}$ & y* & $y *^{++}$ & $y^{0}$ & $y^{0++}$ & $\#$ \\
\hline 1 & 88.0393 & 44.5233 & & & 70.0287 & 35.5180 & S & & & & & & & 12 \\
\hline 2 & 175.0713 & 88.0393 & & & 157.0608 & 79.0340 & $S$ & 1282.6062 & 641.8068 & 81265.5797 & 633.2935 & 1264.5957 & 632.8015 & 11 \\
\hline 3 & 262.1034 & 131.5553 & & & 244.0928 & 122.5500 & $S$ & 1195.5742 & 598.2907 & 71178.5477 & 589.7775 & 1177.5636 & 589.2855 & 10 \\
\hline 4 & 375.1874 & 188.0974 & & & 357.1769 & 179.0921 & L & 1108.5422 & 554.7747 & 1091.5156 & 546.2614 & 1090.5316 & 545.7694 & 9 \\
\hline 5 & 504.2300 & 252.6186 & & & 486.2195 & 243.6134 & $E$ & 995.4581 & 498.2327 & 978.4316 & 489.7194 & 977.4475 & 489.2274 & 8 \\
\hline 6 & 561.2515 & 281.1294 & & & 543.2409 & 272.1241 & G & 866.4155 & 433.7114 & 849.3890 & 425.1981 & 848.4050 & 424.7061 & 7 \\
\hline 7 & 708.3199 & 354.6636 & & & 690.3093 & 345.6583 & $\mathrm{~F}$ & 809.3941 & 405.2007 & 792.3675 & 396.6874 & 791.3835 & 396.1954 & 6 \\
\hline 8 & 845.3788 & 423.1930 & & & 827.3682 & 414.1878 & $\mathrm{H}$ & 662.3257 & 331.6665 & 645.2991 & 323.1532 & 644.3151 & 322.6612 & 5 \\
\hline 9 & 932.4108 & 466.7091 & & & 914.4003 & 457.7038 & S & 525.2667 & 263.1370 & 508.2402 & 254.6237 & 507.2562 & 254.1317 & 4 \\
\hline 10 & 1060.4694 & 530.7383 & 1043.4429 & 522.2251 & 1042.4588 & 521.7331 & $Q$ & 438.2347 & 219.6210 & 421.2082 & 211.1077 & & & 3 \\
\hline 11 & 1223.5327 & 612.2700 & 1206.5062 & 603.7567 & 1205.5222 & 603.2647 & $Y$ & 310.1761 & 155.5917 & 293.1496 & 147.0784 & & & 2 \\
\hline 12 & & & & & & & K & 147.1128 & 74.0600 & 130.0863 & 65.5468 & & & 1 \\
\hline
\end{tabular}
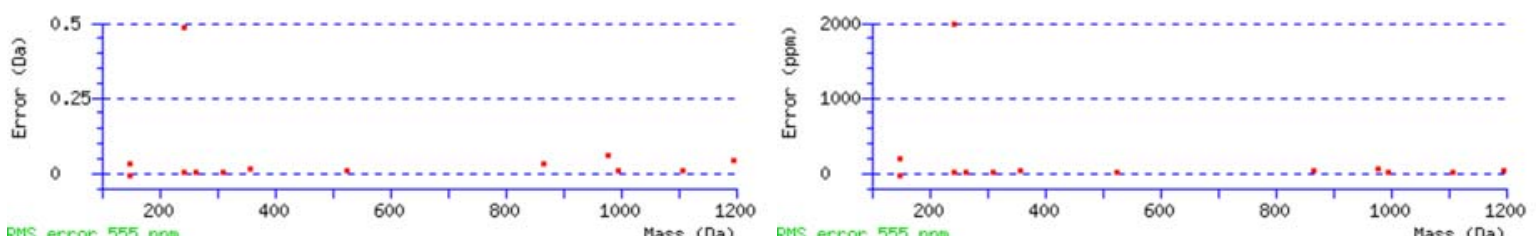

RMS error 555 pph

Mass (Da) RMS error 555 ppm 


\section{Peptide View}

MS/MS Fragmentation of TAAFLLPILSQIYADGPGEALR

Found in gi|6753620|ref|NP_034158.1|, DEAD/H (Asp-Glu-Ala-Asp/His) box polypeptide 3, X-linked [Mus musculus]

Match to Query 812: 2315.440048 from(1158.727300,2+)

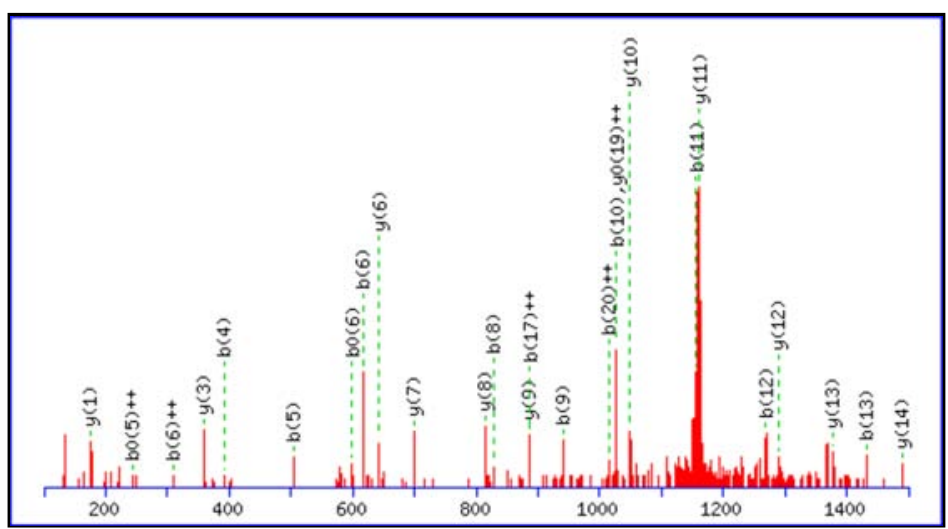

Monoisotopic mass of neutral peptide $\mathrm{Mr}$ (calc): 2315.2525

Ions Score: 64 Expect: 0.002

Matches (Bold Red): 26/226 fragment ions using 64 most intense peaks

\begin{tabular}{|c|c|c|c|c|c|c|c|c|c|c|c|c|c|c|}
\hline \# & b & $b^{++}$ & b* & $b *^{++}$ & $b^{0}$ & $b^{0++}$ & Seq. & $y$ & $y^{++}$ & y* & $y *^{++}$ & $y^{0}$ & $y^{0++}$ & $\#$ \\
\hline 1 & 102.0550 & 51.5311 & & & 84.0444 & 42.5258 & $T$ & & & & & & & 22 \\
\hline 2 & 173.0921 & 87.0497 & & & 155.0815 & 78.0444 & A & 2215.2121 & 1108.1097 & 2198.1855 & 5964 & 2197.2015 & 1099.1044 & 21 \\
\hline 3 & 244.1292 & 122.5682 & & & 226.1186 & 113.5629 & A & 2144.1750 & 1072.5911 & 2127.1484 & 1064.0778 & 2126.1644 & 1063.5858 & 20 \\
\hline 4 & 391.1976 & 196.1024 & & & 373.1870 & 187.0971 & $\mathrm{~F}$ & 2073.1379 & 1037.0726 & 2056.1113 & 1028.5593 & 2055.1273 & 1028.0673 & 19 \\
\hline 5 & 504.2816 & 252.6445 & & & 486.2711 & 243.6392 & $L$ & 1926.0694 & 963.5384 & 1909.0429 & 955.0251 & 1908.0589 & 954.5331 & 18 \\
\hline 6 & 617.3657 & 309.1865 & & & 599.3551 & 300.1812 & $\mathrm{~L}$ & 1812.9854 & 906.9963 & 1795.9588 & 898.4831 & 1794.9748 & 897.9910 & 17 \\
\hline 7 & 714.4185 & 357.7129 & & & 696.4079 & 348.7076 & $P$ & 1699.9013 & 0.4543 & 1682.8748 & .9410 & 1681.8908 & 841.4490 & 16 \\
\hline 8 & 827.5025 & 414.2549 & & & 809.4920 & 405.2496 & I & 1602.8486 & 801.9279 & 1585.8220 & 793.4146 & 1584.8380 & 792.9226 & 15 \\
\hline 9 & 940.5866 & 470.7969 & & & 922.5760 & 461.7916 & L & 1489.7645 & 745.3859 & 1472.7380 & 736.8726 & 1471.7539 & 736.3806 & 14 \\
\hline 10 & 1027.6186 & 514.3129 & & & 1009.6081 & 505.3077 & $S$ & 1376.6804 & 688.8439 & 1359.6539 & 680.3306 & 1358.6699 & 679.8386 & 13 \\
\hline 11 & 1155.6772 & 578.3422 & 1138.6506 & 569.8290 & 1137.6666 & 569.3370 & $Q$ & 1289.6484 & 645.3278 & 1272.6219 & 636.8146 & \begin{tabular}{|l|}
1271.6378 \\
\end{tabular} & 636.3226 & 12 \\
\hline 12 & 1268.7613 & 634.8843 & 1251.7347 & 626.3710 & 1250.7507 & 625.8790 & I & 1161.5898 & 581.2986 & 1144.5633 & 572.7853 & 1143.5793 & 572.2933 & 11 \\
\hline 13 & 1431.8246 & 716.4159 & 1414.7980 & 707.9027 & 1413.8140 & 707.4106 & $Y$ & 1048.5058 & 524.7565 & 1031.4792 & 516.2432 & 1030.4952 & 515.7512 & 10 \\
\hline 14 & 1502.8617 & 751.9345 & 1485.8351 & 743.4212 & 1484.8511 & 742.9292 & A & 885.4424 & 443.2249 & 868.4159 & 434.7116 & 867.4319 & 434.2196 & 9 \\
\hline 15 & 1617.8886 & 809.4480 & 1600.8621 & 800.9347 & 1599.8781 & 800.4427 & D & 814.4053 & 407.7063 & 797.3788 & 399.1930 & 796.3948 & 398.7010 & 8 \\
\hline 16 & 1674.9101 & 837.9587 & 1657.8835 & 829.4454 & 1656.8995 & 828.9534 & $G$ & 699.3784 & 350.1928 & 682.3518 & 341.6796 & 681.3678 & 341.1876 & 7 \\
\hline 17 & 1771.9629 & 886.4851 & 1754.9363 & 877.9718 & 1753.9523 & 877.4798 & $P$ & 642.3569 & 321.6821 & 625.3304 & 313.1688 & 624.3464 & 312.6768 & 6 \\
\hline 18 & 1828.9843 & 914.9958 & 1811.9578 & 906.4825 & 1810.9738 & 905.9905 & $G$ & 545.3042 & 273.1557 & 528.2776 & 264.6424 & 527.2936 & 264.1504 & 5 \\
\hline 19 & 1958.0269 & 979.5171 & 1941.0004 & 971.0038 & 1940.0163 & 970.5118 & $E$ & 488.2827 & 244.6450 & 471.2562 & 236.1317 & 470.2721 & 235.6397 & 4 \\
\hline 20 & 2029.0640 & 1015.0356 & 2012.0375 & 1006.5224 & 2011.0535 & 1006.0304 & A & 359.2401 & 180.1237 & 342.2136 & 171.6104 & & & 3 \\
\hline 21 & 2142.1481 & 1071.5777 & 2125.1215 & 1063.0644 & 2124.1375 & 1062.5724 & L & 288.2030 & 144.6051 & 271.1765 & 136.0919 & & & 2 \\
\hline 22 & & & & & & & $\mathrm{R}$ & 175.1190 & 88.0631 & 158.0924 & 79.5498 & & & 1 \\
\hline
\end{tabular}
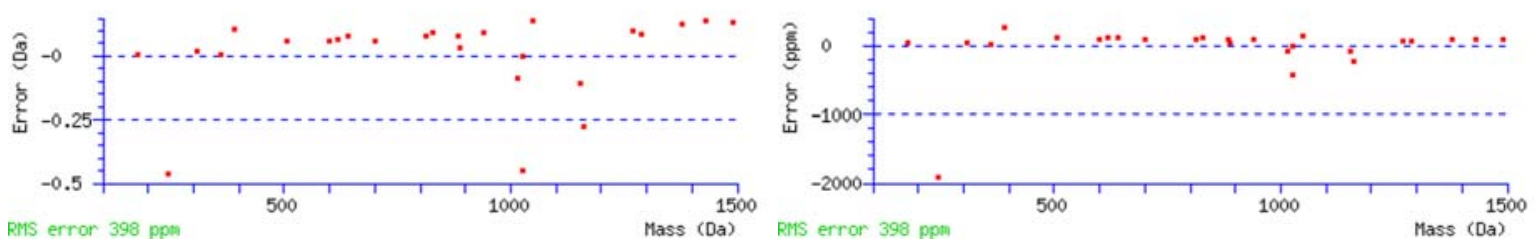


\section{Peptide View}

MS/MS Fragmentation of TAIEAFNETIK

Found in gi|33468921|ref|NP_035215.1|, phosphatidylinositol 3-kinase, regulatory subunit, polypeptide 1 isoform b [Mus musculus]

Match to Query 447: 1235.699248 from(618.856900,2+)

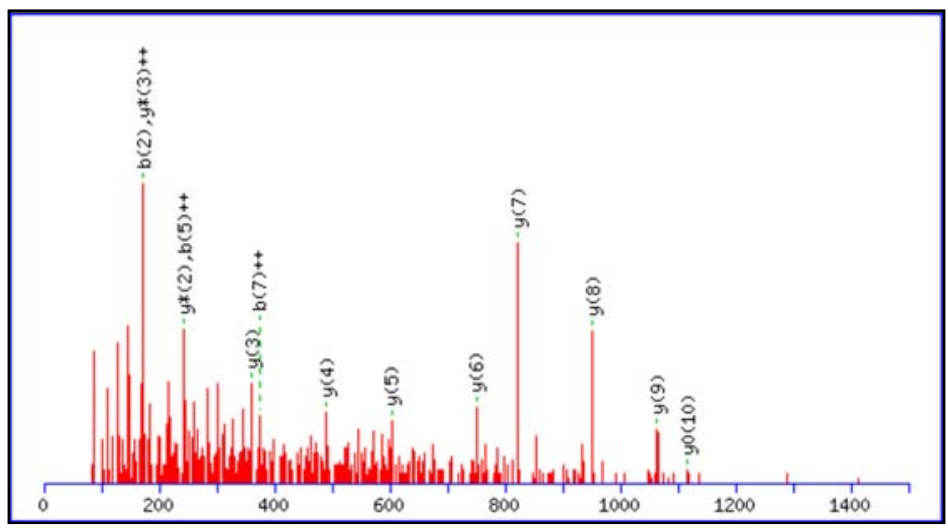

Monoisotopic mass of neutral peptide $\operatorname{Mr}(\mathrm{calc}): 1235.6397$

Ions Score: 64 Expect: 0.00046

Matches (Bold Red): 13/104 fragment ions using 15 most intense peaks

\begin{tabular}{|c|c|c|c|c|c|c|c|c|c|c|c|c|c|c|}
\hline$\#$ & b & $b^{++}$ & b* & $\mathrm{b} *^{++}$ & $b^{0}$ & $b^{0++}$ & Seq. & y & $\mathrm{y}^{++}$ & y* & $y *^{++}$ & $y^{0}$ & $y^{0++}$ & $\#$ \\
\hline 1 & 102.0550 & 51.5311 & & & 84.0444 & 42.5258 & $T$ & & & & & & & 11 \\
\hline 2 & 173.0921 & 87.0497 & & & 155.0815 & 78.0444 & A & 1135.5993 & 568.3033 & 1118.5728 & 559.7900 & 1117.5888 & 559.2980 & 10 \\
\hline 3 & 286.1761 & $\mid 143.5917$ & & & 268.1656 & 134.5864 & I & 1064.5622 & 532.7848 & 1047.5357 & 524.2715 & 1046.5517 & 523.7795 & 9 \\
\hline 4 & 415.2187 & 208.1130 & & & 397.2082 & 199.1077 & $E$ & 951.4782 & 476.2427 & 934.4516 & 467.7294 & 933.4676 & 467.2374 & 8 \\
\hline 5 & 486.2558 & 243.6316 & & & 468.2453 & 234.6263 & A & 822.4356 & 411.7214 & 805.4090 & 403.2082 & 804.4250 & 402.7161 & 7 \\
\hline 6 & 633.3242 & 317.1658 & & & 615.3137 & 308.1605 & $F$ & 751.3985 & 376.2029 & 734.3719 & 367.6896 & 733.3879 & 367.1976 & 6 \\
\hline 7 & 747.3672 & 374.1872 & 730.3406 & 365.6739 & 729.3566 & 365.1819 & $\mathrm{~N}$ & 604.3301 & 302.6687 & 587.3035 & 294.1554 & 586.3195 & 293.6634 & 5 \\
\hline 8 & 876.4098 & 438.7085 & 859.3832 & 430.1952 & 858.3992 & 429.7032 & $E$ & 490.2871 & 245.6472 & 473.2606 & 237.1339 & 472.2766 & 236.6419 & 4 \\
\hline 9 & 977.4574 & 489.2324 & 960.4309 & 480.7191 & 959.4469 & 480.2271 & $T$ & 361.2445 & 181.1259 & 344.2180 & 172.6126 & 343.2340 & 172.1206 & 3 \\
\hline 10 & 1090.5415 & 545.7744 & 1073.5149 & 537.2611 & 1072.5309 & 536.7691 & I & 260.1969 & 130.6021 & 243.1703 & 122.0888 & & & 2 \\
\hline 11 & & & & & & & $\mathrm{~K}$ & 147.1128 & 74.0600 & 130.0863 & 65.5468 & & & 1 \\
\hline
\end{tabular}
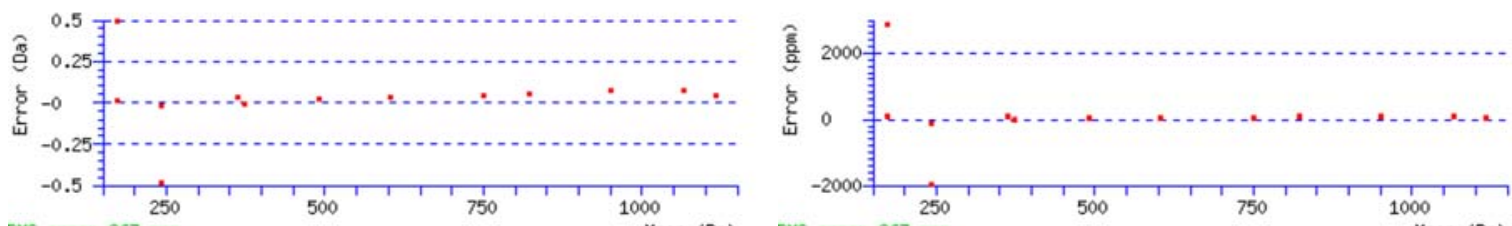

RMS error 967 ppr 


\section{Peptide View}

MS/MS Fragmentation of TEFLSFMNTELAAFTK

Found in gi|21886811|ref|NP_058020.1|, S100 calcium binding protein A11 (calizzarin) [Mus musculus]

Match to Query 605: 1848.976448 from $(925.495500,2+)$

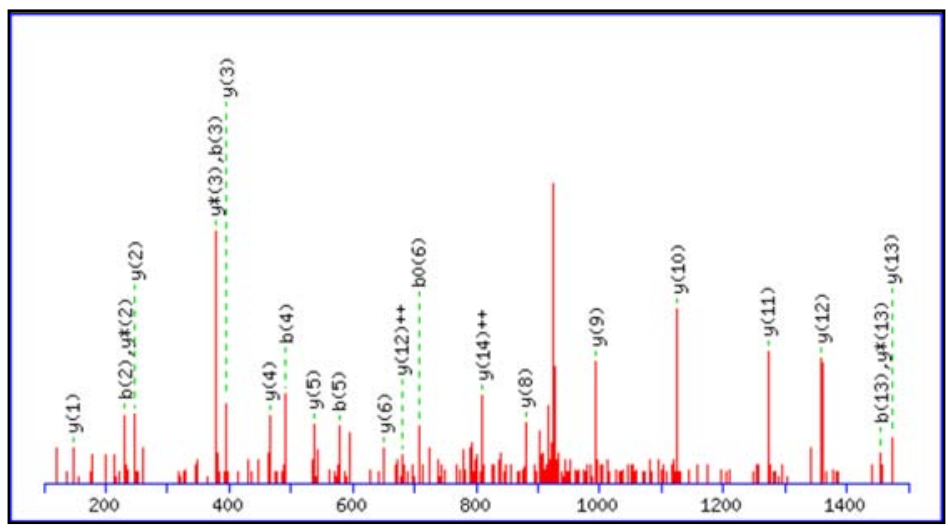

Monoisotopic mass of neutral peptide $\operatorname{Mr}(\mathbf{c a l c}): 1848.8967$

Ions Score: 74 Expect: 0.00012

Matches (Bold Red): 23/164 fragment ions using 45 most intense peaks

\begin{tabular}{|c|c|c|c|c|c|c|c|c|c|c|c|c|c|c|}
\hline$\#$ & b & $b^{++}$ & $b *$ & $b *^{++}$ & $b^{0}$ & $b^{0++}$ & Seq. & y & $\mathrm{y}^{++}$ & y* & $y *^{++}$ & $y^{0}$ & $y^{0++}$ & $\#$ \\
\hline 1 & 102.0550 & 51.5311 & & & 84.0444 & 42.5258 & $T$ & & & & & & & 16 \\
\hline 2 & 231.0975 & 116.0524 & & & 213.0870 & 107.0471 & $\mathrm{E}$ & 748.8564 & 874.9318 & 1731.8298 & 866 & 1730 & 865.9 & 15 \\
\hline 3 & 378.1660 & 189.5866 & & & 60.1554 & 180 & $\mathrm{~F}$ & 1619.8138 & 810.4105 & 1602.7872 & 801.8972 & 1601.8032 & 801.4052 & 14 \\
\hline 4 & 1.2500 & 246.1286 & & & 3.2395 & 237.1234 & L & 1472.7454 & 736.8763 & 1455.7188 & 728.3630 & 1454.7348 & 727.8710 & 13 \\
\hline 5 & 578.2820 & 289.6447 & & & 560.2715 & 280.6394 & $S$ & 1359.6613 & 680.3343 & 1342.6347 & 671.8210 & 1341.6507 & 671.3290 & 12 \\
\hline 6 & 725.3505 & 363.1789 & & & 707.3399 & 354.1736 & $F$ & 1272.6293 & 183 & 125 & 050 & 1254.6187 & 627.8130 & 11 \\
\hline 7 & 856.3909 & 428.6991 & & & 838.3804 & 419.6938 & $M$ & 1125.5609 & 563.2841 & 1108.5343 & 554.7708 & 1107.5503 & 554.2788 & 10 \\
\hline 8 & 970.4339 & 485.7206 & 0.1 & 4 & 952.4233 & 476.7153 & $\mathrm{~N}$ & 994.5204 & 497.7638 & 977.4938 & 489.2505 & 976.5098 & 488.7585 & 9 \\
\hline 9 & 1071.4815 & 536.2444 & 1054.4550 & 527.7311 & 1053.4710 & 527.2391 & $T$ & 880.4774 & 440.7424 & 863.4509 & 432.2291 & 862.4669 & 431.7371 & 8 \\
\hline 10 & 1200.5241 & 600.7657 & 1183.4976 & 592.2524 & 1182.5136 & 591.7604 & $E$ & 779.4298 & 390.2185 & 762.4032 & 381.7052 & 761.4192 & 381.2132 & 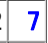 \\
\hline 11 & 1313.6082 & 657.3077 & 1296.5816 & 648.7945 & 1295.5976 & 648.3025 & L & 650.3872 & 325.6972 & & 317.1839 & 632.3766 & 316.6919 & 6 \\
\hline 12 & 1384.6453 & 692.8263 & 1367.6188 & 684.3130 & 1366.6347 & 683.8210 & A & 537.3031 & 269.1552 & 20.2766 & 260.6419 & 519.2925 & 260.1499 & 5 \\
\hline 13 & 1455.6824 & 728.3448 & 1438.6559 & 719.8316 & 1437.6719 & 719.3396 & A & 466.2660 & 233.6366 & 449.2395 & 225.1234 & 448.2554 & 224.6314 & 4 \\
\hline 14 & 1602.7508 & 801.8791 & 1585.7243 & 793.3658 & 1584.7403 & 792.8738 & $\mathrm{~F}$ & 395.2289 & 198.1181 & 378.2023 & 189.6048 & 377.2183 & 189.1128 & 3 \\
\hline 15 & 1703.7985 & 852.4029 & 1686.7720 & 843.8896 & 1685.7879 & 843.3976 & $\mathrm{~T}$ & 248.1605 & 124.5839 & 231.1339 & 116.0706 & 230.1499 & 115.5786 & 2 \\
\hline 16 & & & & & & & $\mathrm{~K}$ & 147.1128 & 74.0600 & 130.0863 & 65.5468 & & & 1 \\
\hline
\end{tabular}
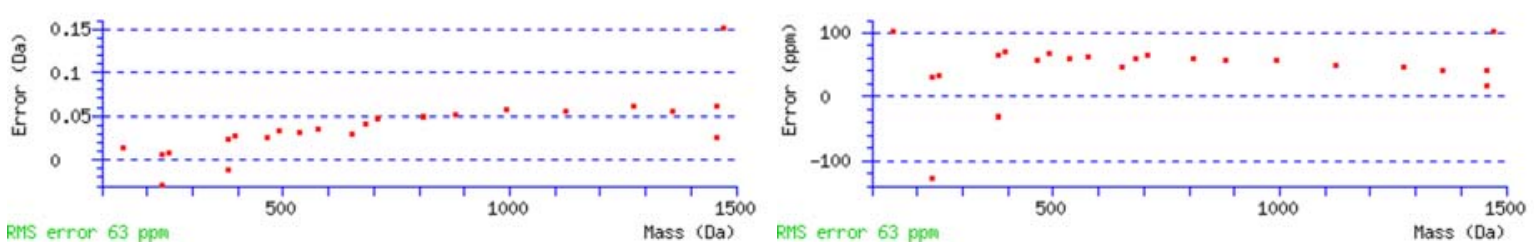


\section{Peptide View}

MS/MS Fragmentation of TGTFIVIDILIDIIR

Found in gi|6755228|ref|NP_035332.1|, protein tyrosine phosphatase, non-receptor type 11 [Mus musculus]

Match to Query 561: 1701.081648 from $\left(851.548100,2^{+}\right)$

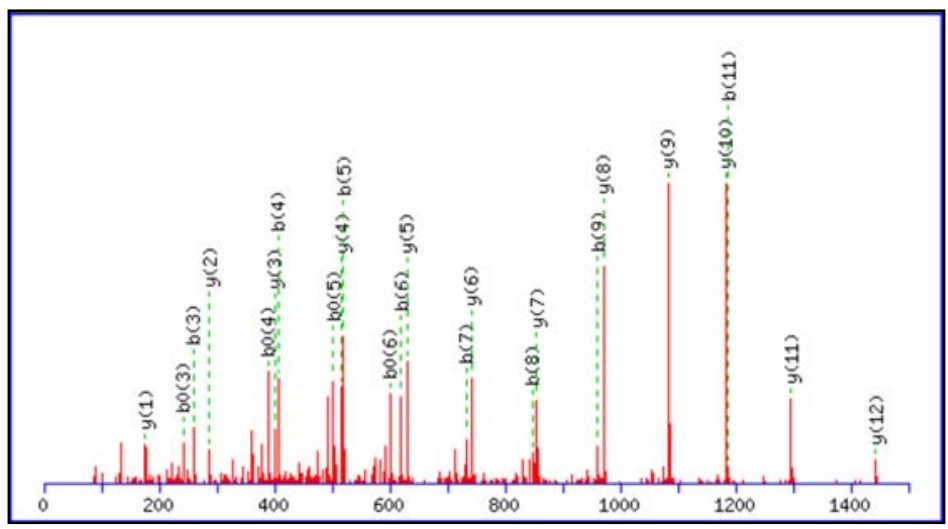

Monoisotopic mass of neutral peptide $\mathrm{Mr}$ (calc): 1701.0076

Ions Score: 85 Expect: $1.2 \mathrm{e}-005$

Matches (Bold Red): 24/134 fragment ions using 44 most intense peaks

\begin{tabular}{|c|c|c|c|c|c|c|c|c|c|c|c|c|}
\hline \# & b & $b^{++}$ & $b^{0}$ & $b^{0++}$ & Seq. & y & $y^{++}$ & y* & $y *^{++}$ & $y^{0}$ & $y^{0++}$ & $\#$ \\
\hline 1 & 102.0550 & 51.5311 & 84.0444 & 42.5258 & $T$ & & & & & & & 15 \\
\hline 2 & 159.0764 & 80.0418 & 141.0659 & 71.0366 & G & 1600.9672 & 800.9872 & 1583.9407 & 792.4740 & 158 & 791. & 14 \\
\hline 3 & 260.1241 & 130.5657 & 242.1135 & 121.5604 & $\mathrm{~T}$ & 1543.9458 & 772.4765 & 1526.9192 & 763.9632 & 1525.9352 & 763.4712 & 13 \\
\hline 4 & 407.1925 & 204.0999 & 389.1819 & 195.0946 & $F$ & 1442.8981 & 721.9527 & 1425.8715 & 713.4394 & 1424.8875 & 712.9474 & 12 \\
\hline 5 & 520.2766 & 260.6419 & 502.2660 & 251.6366 & I & 1295.8297 & 648.4185 & 1278.8031 & 639.9052 & 1277.8191 & 639.4132 & 11 \\
\hline 6 & 619.3450 & 310.1761 & 601.3344 & 301.1708 & V & 1182.7456 & 591.8764 & 1165.7191 & 583.3632 & 1164.7350 & 582.8712 & 10 \\
\hline 7 & 732.4290 & 366.7182 & 714.4185 & 357.7129 & I & 1083.6772 & 542.3422 & 1066.6506 & 533.8290 & 1065.6666 & 533.3370 & 9 \\
\hline 8 & 847.4560 & 424.2316 & 829.4454 & 415.2263 & D & 970.5931 & 485.8002 & 953.5666 & 477.2869 & 952.5826 & 476.7949 & 8 \\
\hline 9 & 960.5400 & 480.7737 & 942.5295 & 471.7684 & I & 855.5662 & 428.2867 & 838.5396 & 419.7735 & 837.5556 & 419.2815 & 7 \\
\hline 10 & 1073.6241 & 537.3157 & 1055.6135 & 528.3104 & L & 742.4821 & 371.7447 & 725.4556 & 363.2314 & 724.4716 & 362.7394 & 6 \\
\hline 11 & 1186.7082 & 593.8577 & 1168.6976 & 584.8524 & I & 629.3981 & 315.2027 & 612.3715 & 306.6894 & 611.3875 & 306.1974 & 5 \\
\hline 12 & 1301.7351 & 651.3712 & 1283.7245 & 642.3659 & D & 516.3140 & 258.6606 & 499.2875 & 250.1474 & 498.3034 & 249.6554 & 4 \\
\hline 13 & 1414.8192 & 707.9132 & 1396.8086 & 698.9079 & I & 401.2871 & 201.1472 & 384.2605 & 192.6339 & & & 3 \\
\hline 14 & 1527.9032 & 764.4552 & 1509.8927 & 755.4500 & I & 288.2030 & 144.6051 & 271.1765 & 136.0919 & & & 2 \\
\hline 15 & & & & & $\mathrm{R}$ & 175.1190 & 88.0631 & 158.0924 & 79.5498 & & & 1 \\
\hline
\end{tabular}

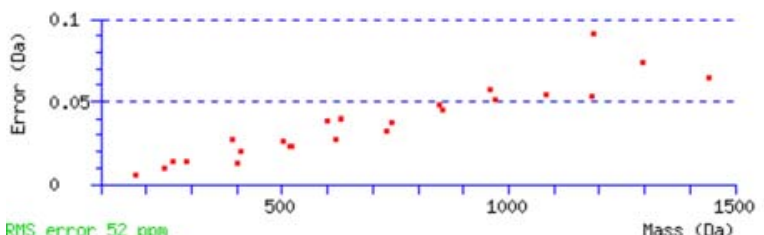

RMS error 52 ppo

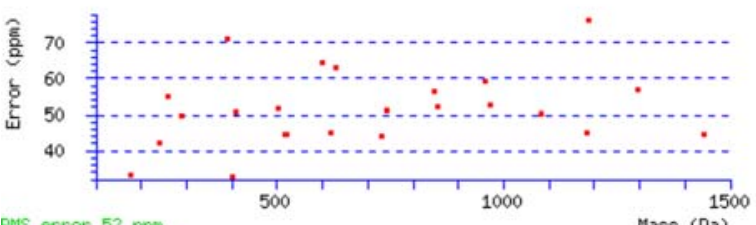

Mass (Da) 


\section{Peptide View}

MS/MS Fragmentation of TITELADWLVLIDQMLK

Found in gi|46575908|ref|NP_035812.2|, utrophin [Mus musculus]

Match to Query 658: 2001.117448 from(1001.566000,2+)

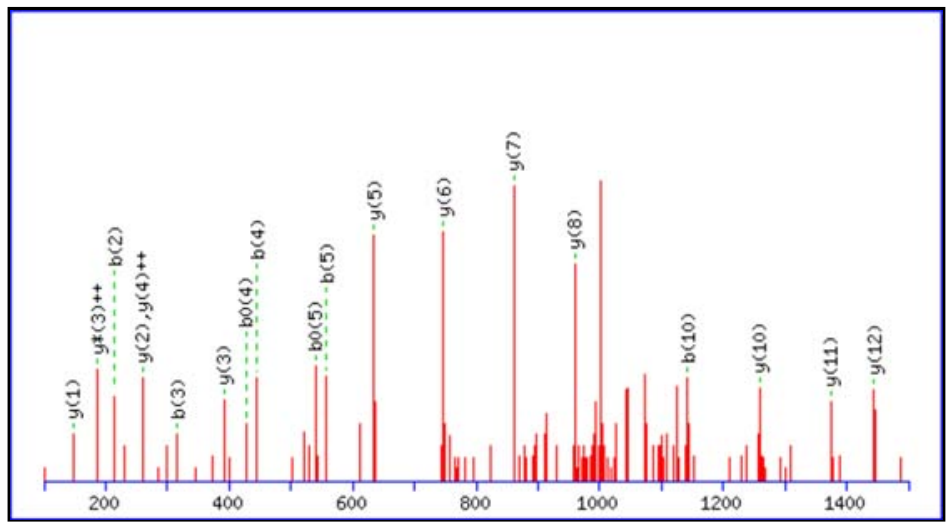

Monoisotopic mass of neutral peptide $\operatorname{Mr}(\mathrm{calc}): 2001.0856$

Ions Score: 67 Expect: 0.00083

Matches (Bold Red): 19/158 fragment ions using 28 most intense peaks

\begin{tabular}{|c|c|c|c|c|c|c|c|c|c|c|c|c|c|c|}
\hline$\#$ & b & $b^{++}$ & b* & $\mathrm{b} *^{++}$ & $b^{0}$ & $b^{0++}$ & Seq. & $y$ & $y^{++}$ & y* & $y *^{++}$ & $y^{0}$ & $y^{0++}$ & $\#$ \\
\hline 1 & 102.0550 & 51.5311 & & & 84.0444 & 42.5258 & $T$ & & & & & & & 17 \\
\hline 2 & 215.1390 & 108.0731 & & & 197.1285 & 99.0679 & I & 1901.0452 & 951.0262 & 1884.0187 & 942.5130 & 1883.0346 & 942.0210 & 16 \\
\hline 3 & 316.1867 & 158.5970 & & & 298.1761 & 149.5917 & $T$ & 1787.9611 & 894.4842 & 1770.9346 & \begin{tabular}{|l|}
885.9709 \\
\end{tabular} & 1769.9506 & 885.4789 & 15 \\
\hline 4 & 445.2293 & 223.1183 & & & 427.2187 & 214.1130 & $E$ & 1686.9135 & 843.9604 & 1669.8869 & 835.4471 & 1668.9029 & 834.9551 & 14 \\
\hline 5 & 558.3133 & 279.6603 & & & 540.3028 & 270.6550 & L & 1557.8709 & 779.4391 & 1540.8443 & 770.9258 & 1539.8603 & 770.4338 & 13 \\
\hline 6 & 629.3505 & 315.1789 & & & 611.3399 & 306.1736 & A & 1444.7868 & 722.8970 & 1427.7603 & \begin{tabular}{|l|}
714.3838 \\
\end{tabular} & 1426.7762 & 713.8918 & 12 \\
\hline 7 & 744.3774 & 372.6923 & & & 726.3668 & 363.6871 & D & 1373.7497 & 687.3785 & 1356.7232 & 678.8652 & 1355.7391 & 678.3732 & 11 \\
\hline 8 & 930.4567 & 465.7320 & & & 912.4461 & 456.7267 & W & 1258.7228 & 629.8650 & 1241.6962 & 621.3517 & 1240.7122 & 620.8597 & 10 \\
\hline 9 & 1043.5408 & 522.2740 & & & 1025.5302 & 513.2687 & L & 1072.6435 & 536.8254 & 1055.6169 & 528.3121 & 1054.6329 & 527.8201 & 9 \\
\hline 10 & 1142.6092 & 571.8082 & & & 1124.5986 & 562.8029 & V & 959.5594 & 480.2833 & 942.5328 & 471.7701 & 941.5488 & 471.2781 & 8 \\
\hline 11 & 1255.6932 & 628.3503 & & & 1237.6827 & 619.3450 & L & 860.4910 & 430.7491 & 843.4644 & 422.2359 & 842.4804 & 421.7438 & 7 \\
\hline 12 & 1368.7773 & 684.8923 & & & 1350.7667 & 675.8870 & I & 747.4069 & 374.2071 & 730.3804 & 365.6938 & \begin{tabular}{|l|l|} 
& 729.3964 \\
\end{tabular} & 365.2018 & 6 \\
\hline 13 & 1483.8042 & 742.4058 & & & 1465.7937 & 733.4005 & D & 634.3229 & 317.6651 & 617.2963 & 309.1518 & 616.3123 & 308.6598 & 5 \\
\hline 14 & 1611.8628 & 806.4350 & 1594.8363 & 797.9218 & 1593.8523 & 797.4298 & $Q$ & 519.2959 & 260.1516 & 502.2694 & 251.6383 & & & 4 \\
\hline 15 & 1742.9033 & 871.9553 & 1725.8767 & 863.4420 & 1724.8927 & 862.9500 & $M$ & 391.2373 & \begin{tabular}{|l|}
196.1223 \\
\end{tabular} & 374.2108 & 187.6090 & & & 3 \\
\hline 16 & 1855.9874 & 928.4973 & 1838.9608 & 919.9840 & 1837.9768 & 919.4920 & L & 260.1969 & 130.6021 & 243.1703 & 122.0888 & & & 2 \\
\hline 17 & & & & & & & $\mathrm{~K}$ & 147.1128 & 74.0600 & 130.0863 & 65.5468 & & & 1 \\
\hline
\end{tabular}
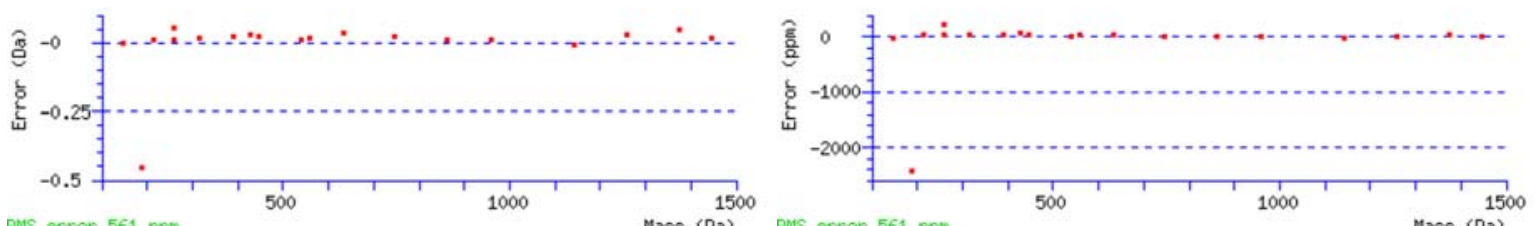

RMS error 561 ppm

Mass (Da) RMS error 561 ppen

Mass (Da) 


\section{Peptide View}

MS/MS Fragmentation of TLGSGAFGQVVEATAHGLSHSQATMK

Found in gi|6679259|ref|NP_032835.1|, platelet derived growth factor receptor, beta polypeptide [Mus musculus]

Match to Query 769: 2584.262472 from $(862.428100,3+)$

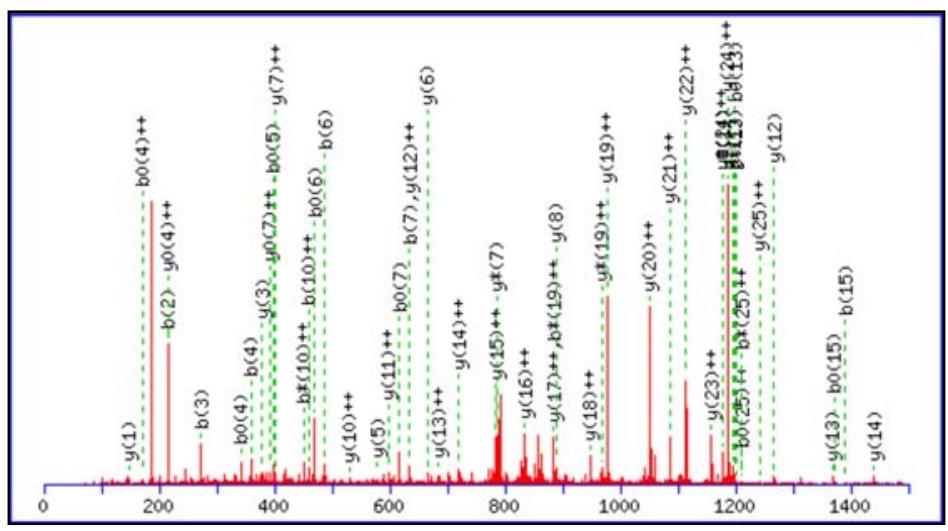

Monoisotopic mass of neutral peptide $\mathrm{Mr}$ (calc): 2584. 2703

Ions Score: 57 Expect: 0.016

Matches (Bold Red): 52/280 fragment ions using 171 most intense peaks

\begin{tabular}{|c|c|c|c|c|c|c|c|c|c|c|c|c|c|c|}
\hline \# & b & $b^{++}$ & $b *$ & $b *^{++}$ & $b^{0}$ & $b^{0++}$ & Seq. & y & $y^{++}$ & y* & $y *^{++}$ & $y^{0}$ & $y^{0++}$ & $\#$ \\
\hline 1 & 102.0550 & 51.5311 & & & 84.0444 & 42.5258 & $T$ & & & & & & & 26 \\
\hline 2 & 215.1390 & 108.0731 & & & 97.1285 & 99.0679 & $L$ & 34.2299 & 1242.6186 & 2467.2034 & 1234 & 2194 & 133 & 25 \\
\hline 3 & 2.1605 & 136.5 & & & 99 & 127.5786 & G & 11.1459 & 1186.0766 & 2354.1193 & 1177.5633 & 2353.1353 & 1177.0713 & 24 \\
\hline 4 & 9.1925 & 180.0999 & & & 1.1819 & 171.0946 & $\mathrm{~s}$ & 314.1244 & 1157.5658 & 2297.0979 & 1149.0526 & 2296.1138 & 1148.5606 & 23 \\
\hline 5 & 416.2140 & 208.6106 & & & 398.2034 & 199.6053 & G & 2227.0924 & 1114.0498 & 2210.0658 & 1105.5366 & 2209.0818 & 1105.0445 & 22 \\
\hline 6 & 487.2511 & 244.1292 & & & 469.2405 & 235.1239 & A & 2170.0709 & 1085.5391 & 2153.0444 & 1077.0258 & 2152.0604 & 1076.5338 & 21 \\
\hline 7 & 634.3195 & 317.6634 & & & 616.3089 & 308.6581 & $\mathrm{~F}$ & 2099.0338 & 1050.0205 & 2082.0073 & 1041.5073 & 2081.0232 & 1041.0153 & 20 \\
\hline 8 & 691.3409 & 346.1741 & & & .3304 & 337.1688 & G & 1951.9654 & 6.4863 & 1934.9389 & 967.9731 & 1933.9548 & 967.4811 & 19 \\
\hline 9 & 819.3995 & 410.2034 & 802.3730 & 401.6901 & 801.3890 & 401.1981 & $\mathbf{Q}$ & 1894.9439 & 947.9756 & 1877.9174 & 939.4623 & 1876.9334 & 938.9703 & 18 \\
\hline 0 & 918.4679 & 459.7376 & 901.4414 & 1.2243 & 900.4574 & 450.7323 & V & 1766.8854 & 3.9463 & 174 & 5.4330 & 1748.8748 & 874.9410 & 17 \\
\hline 11 & 1017.5363 & 509.2718 & 1000.5098 & 500.7585 & 999.5258 & 500.2665 & V & 1667.8170 & 834.4121 & 1650.7904 & 825.8988 & 1649.8064 & 825.4068 & 16 \\
\hline 2 & 1146.5789 & 3.7931 & 1129.5524 & 565.2798 & 1128.5684 & 564.7878 & $E$ & 1568.7485 & 84.8779 & 1551.7220 & 776.3646 & 1550.7380 & 775.8726 & 15 \\
\hline 13 & 1217.6160 & 609.3117 & 1200.5895 & 600.7984 & 1199.6055 & 600.3064 & A & 1439.7060 & 720.3566 & 1422.6794 & 711.8433 & 1421.6954 & 711.3513 & 14 \\
\hline 14 & 1318.6637 & 659.8355 & 1301.6372 & 651.3222 & 1300.6532 & 650.8302 & $T$ & 1368.6688 & 684.8381 & 1351.6423 & 676.3248 & 1350.6583 & 675.8328 & 13 \\
\hline 15 & 1389.7008 & 695.3541 & 1372.6743 & 686.8408 & 1371.6903 & 686.3488 & A & 1267.6212 & 34.3142 & 1250.5946 & 625.8009 & 1249.6106 & 625.3089 & 12 \\
\hline 16 & 1526.7597 & 763.8835 & 1509.7332 & 755.3702 & 1508.7492 & 754.8782 & $\mathrm{H}$ & 1196.5841 & 598.7957 & 1179.5575 & 590.2824 & 1178.5735 & 589.7904 & 11 \\
\hline 17 & 1583.7812 & 792.3942 & 1566.7547 & 783.8810 & 1565.7706 & 783.3890 & G & 1059.5251 & 530.2662 & 1042.4986 & 521.7529 & 1041.5146 & 521.2609 & 10 \\
\hline 18 & 1696.8653 & 848.9363 & 1679.8387 & 840.4230 & 1678.8547 & 839.9310 & L & 1002.5037 & 501.7555 & 985.4771 & 493.2422 & 984.4931 & 492.7502 & 9 \\
\hline 19 & 1783.8973 & 892.4523 & 1766.8707 & 883.9390 & 1765.8867 & 883.4470 & S & 889.4196 & 445.2134 & 872.3931 & 436.7002 & 871.4091 & 436.2082 & 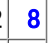 \\
\hline 20 & 1920.9562 & 960.9817 & 1903.9297 & 952.4685 & 1902.9456 & 951.9765 & $\mathrm{H}$ & 802.3876 & 401.6974 & 785.3610 & 393.1842 & 784.3770 & 392.6922 & 2 \\
\hline 21 & 2007.9882 & 1004.4978 & 1990.9617 & 995.9845 & 1989.9777 & 995.4925 & S & 665.3287 & 333.1680 & 648.3021 & 324.6547 & 647.3181 & 324.1627 & 6 \\
\hline 22 & 2136.0468 & 1068.5270 & 2119.0203 & 1060.0138 & 2118.0363 & 1059.5218 & $Q$ & 578.2967 & 289.6520 & 561.2701 & 281.1387 & 560.2861 & 280.6467 & 5 \\
\hline 23 & 2207.0839 & 1104.0456 & 2190.0574 & 1095.5323 & 2189.0734 & 1095.0403 & A & 450.2381 & 225.6227 & 433.2115 & 217.1094 & 432.2275 & 216.6174 & 4 \\
\hline 24 & 2308.1316 & 1154.5694 & 2291.1051 & 1146.0562 & 2290.1210 & 1145.5642 & $T$ & 379.2010 & 190.1041 & 362.1744 & 181.5908 & 361.1904 & 181.0988 & 3 \\
\hline 25 & 2439.1721 & 1220.0897 & 2422.1455 & 1211.5764 & \begin{tabular}{|l|}
2421.1615 \\
\end{tabular} & 1211.0844 & M & 278.1533 & 139.5803 & 261.1267 & 131.0670 & & & 2 \\
\hline 26 & & & & & & & $\mathrm{~K}$ & 147.1128 & 74.0600 & 130.0863 & 65.5468 & & & 1 \\
\hline
\end{tabular}
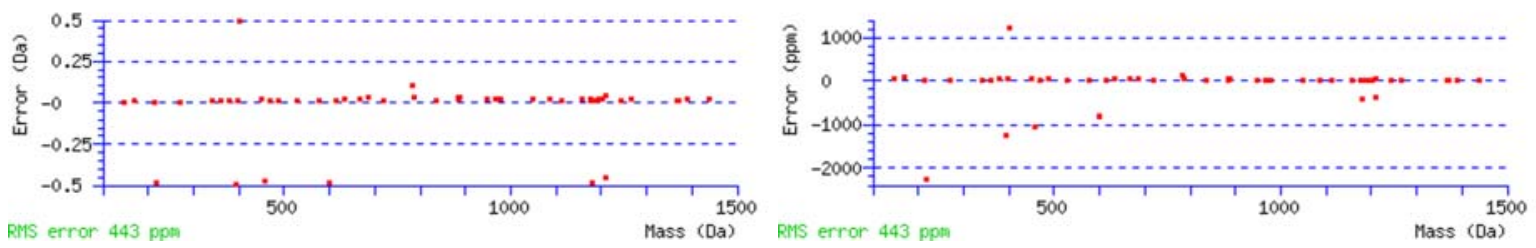


\section{Peptide View}

MS/MS Fragmentation of TLVTQNSGVEALIHAILR

Found in gi|28395018|ref|NP_034723.1|, junction plakoglobin [Mus musculus]

Match to Query 1073: 1940.228472 from(647.750100,3+)

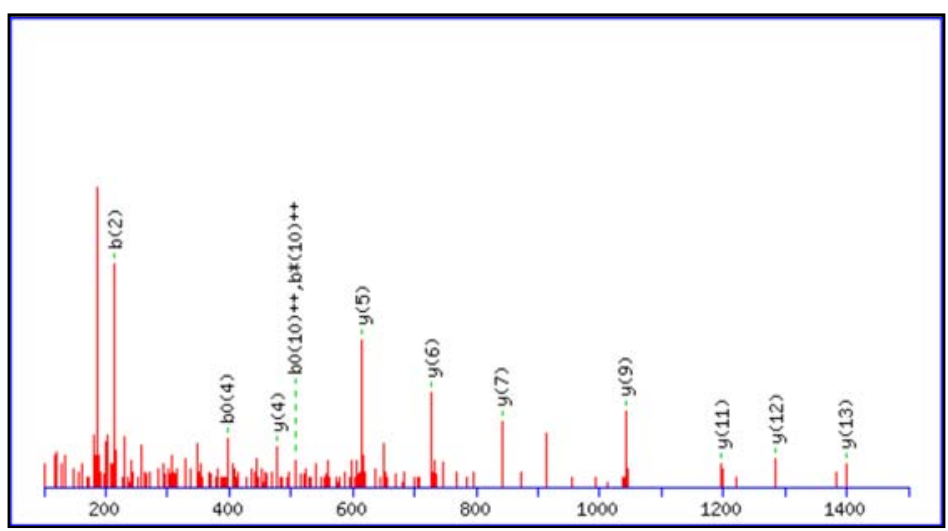

Monoisotopic mass of neutral peptide $\mathrm{Mr}$ (calc): 1940.1150

Variable modifications:

R18 : Arginine-13C6 (R-13C6)

Ions Score: 53 Expect: 0.02

Matches (Bold Red): 12/180 fragment ions using 16 most intense peaks

\begin{tabular}{|c|c|c|c|c|c|c|c|c|c|c|c|c|c|c|}
\hline$\#$ & b & $\mathrm{b}^{++}$ & $b *$ & $b *^{++}$ & $b^{0}$ & $b^{0++}$ & Seq. & $y$ & $\mathrm{y}^{++}$ & y* & $y *^{++}$ & $y^{0}$ & $y^{0++}$ & $\#$ \\
\hline 1 & 102.0550 & 51.5311 & & & 84.0444 & 42.5258 & $T$ & & & & & & & 18 \\
\hline 2 & 215.1390 & 108.0731 & & & 197.1285 & 99.0679 & L & 1840.0746 & 920.5409 & 1823.0480 & 912.0277 & 1822.0640 & 911.5357 & 17 \\
\hline 3 & 314.2074 & 157.6074 & & & 296.1969 & 148.6021 & V & 1726.9905 & 863.9989 & 1709.9640 & 855.4856 & 1708.9800 & 854.9936 & 16 \\
\hline 4 & 415.2551 & 208.1312 & & & 397.2445 & 199.1259 & $T$ & 1627.9221 & 814.4647 & 1610.8956 & 805.9514 & 1609.9116 & 805.4594 & 15 \\
\hline 5 & 543.3137 & 272.1605 & 526.2871 & 263.6472 & 525.3031 & 263.1552 & $Q$ & 1526.8744 & 763.9409 & 1509.8479 & 755.4276 & 1508.8639 & 754.9356 & 14 \\
\hline 6 & 657.3566 & 329.1819 & 640.3301 & 320.6687 & 639.3461 & 320.1767 & $\mathrm{~N}$ & 1398.8159 & 699.9116 & 1381.7893 & 691.3983 & 1380.8053 & 690.9063 & 13 \\
\hline 7 & 744.3886 & 372.6980 & 727.3621 & \begin{tabular}{|l|}
364.1847 \\
\end{tabular} & 726.3781 & 363.6927 & S & 1284.7729 & 642.8901 & 1267.7464 & 634.3768 & 1266.7624 & 633.8848 & 12 \\
\hline 8 & 801.4101 & 401.2087 & 784.3836 & 392.6954 & 783.3995 & 392.2034 & G & 1197.7409 & 599.3741 & 1180.7144 & 590.8608 & 1179.7303 & 590.3688 & 11 \\
\hline 9 & 900.4785 & 450.7429 & 883.4520 & 442.2296 & 882.4680 & 441.7376 & V & 1140.7194 & 570.8634 & 1123.6929 & 562.3501 & 1122.7089 & 561.8581 & 10 \\
\hline 10 & 1029.5211 & 515.2642 & 1012.4946 & 506.7509 & 1011.5105 & 506.2589 & $\mathrm{E}$ & 1041.6510 & 521.3292 & 1024.6245 & 512.8159 & 1023.6405 & 512.3239 & 9 \\
\hline 11 & 1100.5582 & 550.7827 & \begin{tabular}{|l|}
1083.5317 \\
\end{tabular} & 542.2695 & 1082.5477 & 541.7775 & A & 912.6084 & 456.8079 & 895.5819 & 448.2946 & & & 8 \\
\hline 12 & 1213.6423 & 607.3248 & 1196.6157 & 598.8115 & 1195.6317 & 598.3195 & $\mathrm{~L}$ & 841.5713 & 421.2893 & 824.5448 & 412.7760 & & & 7 \\
\hline 13 & 1326.7263 & 663.8668 & 1309.6998 & 655.3535 & 1308.7158 & 654.8615 & I & 728.4873 & 364.7473 & 711.4607 & 356.2340 & & & 6 \\
\hline 14 & 1463.7852 & 732.3963 & 1446.7587 & 723.8830 & 1445.7747 & 723.3910 & $\mathrm{H}$ & 615.4032 & 308.2052 & 598.3767 & 299.6920 & & & 5 \\
\hline 15 & 1534.8224 & 767.9148 & 1517.7958 & 759.4015 & 1516.8118 & 758.9095 & A & 478.3443 & 239.6758 & 461.3178 & 231.1625 & & & 4 \\
\hline 16 & 1647.9064 & 824.4568 & 1630.8799 & 815.9436 & 1629.8959 & 815.4516 & I & 407.3072 & 204.1572 & 390.2806 & 195.6440 & & & 3 \\
\hline 17 & 1760.9905 & 880.9989 & 1743.9639 & 872.4856 & 1742.9799 & 871.9936 & L & 294.2231 & 147.6152 & 277.1966 & 139.1019 & & & 2 \\
\hline 18 & & & & & & & $\mathrm{R}$ & 181.1391 & 91.0732 & 164.1125 & 82.5599 & & & 1 \\
\hline
\end{tabular}
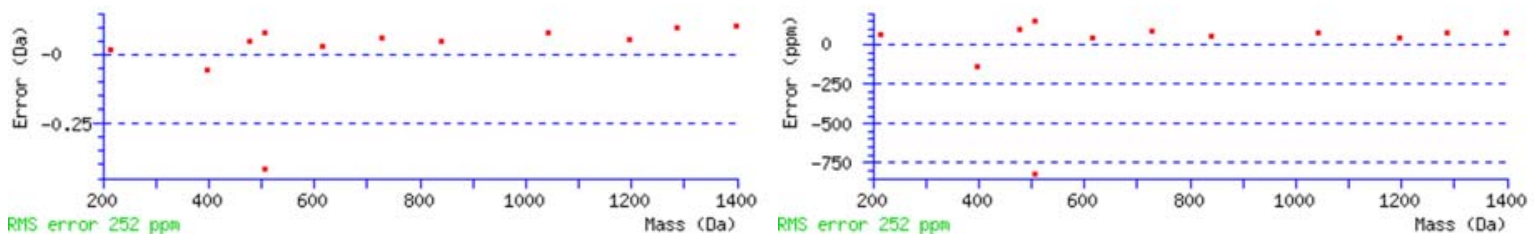


\section{Peptide View}

MS/MS Fragmentation of TPFSLVGNVFELNFK

Found in gi|34996495|ref|NP_062616.2|, ribophorin II [Mus musculus]

Match to Query 408: 1710.992248 from $(856.503400,2+)$

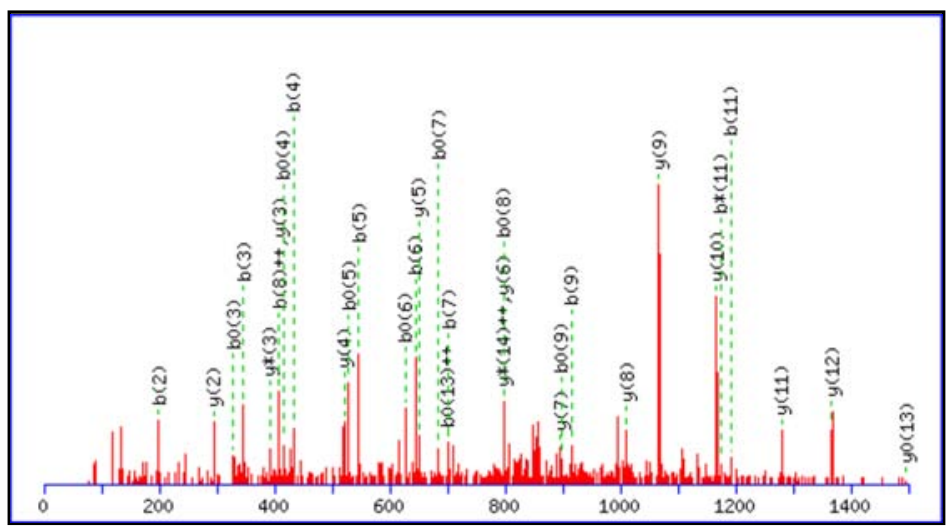

Monoisotopic mass of neutral peptide $\operatorname{Mr}$ (calc): 1710.8980

Ions Score: 52 Expect: 0.019

Matches (Bold Red): 32/146 fragment ions using 79 most intense peaks

\begin{tabular}{|c|c|c|c|c|c|c|c|c|c|c|c|c|c|c|}
\hline$\#$ & b & $b^{++}$ & $b *$ & $b *^{++}$ & $b^{0}$ & $b^{0++}$ & Seq. & y & $y^{++}$ & y* & $y *^{++}$ & $y^{0}$ & $y^{0++}$ & $\#$ \\
\hline 1 & 102.0550 & 51.5311 & & & 84.0444 & 42.5258 & $T$ & & & & & & & 15 \\
\hline 2 & 199.1077 & 100.0575 & & & 181.0972 & 91.0522 & $P$ & 1610.8577 & 805.9325 & 1593.8311 & 797.4192 & 1592.8471 & 796.9272 & 14 \\
\hline 3 & 346.1761 & 173.5917 & & & 328.1656 & 164.5864 & $\mathrm{~F}$ & 1513.8049 & 757.4061 & 1496.7784 & 748.8928 & 1495.7943 & 748.4008 & 13 \\
\hline 4 & 433.2082 & 217.1077 & & & 415.1976 & 208.1024 & $S$ & 1366.7365 & 683.8719 & 1349.7100 & 675.3586 & 1348.7259 & 674.8666 & 12 \\
\hline 5 & 546.2922 & 273.6497 & & & 528.2817 & 264.6445 & L & 1279.7045 & 640.3559 & 1262.6779 & 631.8426 & 1261.6939 & 631.3506 & 11 \\
\hline 6 & 645.3606 & 323.1840 & & & 627.3501 & 314.1787 & V & 1166.6204 & 583.8138 & 1149.5939 & 575.3006 & 1148.6098 & 574.8086 & 10 \\
\hline 7 & 702.3821 & 351.6947 & & & 684.3715 & 342.6894 & G & 1067.5520 & 534.2796 & 1050.5255 & 525.7664 & 1049.5414 & 525.2744 & 9 \\
\hline 8 & 816.4250 & 408.7161 & 799.3985 & 400.2029 & 798.4145 & 399.7109 & $\mathrm{~N}$ & 1010.5305 & 505.7689 & 993.5040 & 497.2556 & 992.5200 & 496.7636 & 8 \\
\hline 9 & 915.4934 & 458.2504 & 898.4669 & 449.7371 & 897.4829 & 449.2451 & V & 896.4876 & 448.7474 & 879.4611 & 440.2342 & 878.4770 & 439.7422 & 7 \\
\hline 10 & 1062.5618 & 531.7846 & 1045.5353 & 523.2713 & 1044.5513 & \begin{tabular}{|l|}
522.7793 \\
\end{tabular} & $F$ & 797.4192 & 399.2132 & 780.3927 & 390.7000 & 779.4086 & 390.2080 & 6 \\
\hline 11 & 1191.6044 & 596.3059 & 1174.5779 & 587.7926 & 1173.5939 & 587.3006 & $E$ & 650.3508 & 325.6790 & 633.3242 & 317.1658 & 632.3402 & 316.6738 & 5 \\
\hline 12 & 1304.6885 & 652.8479 & 1287.6619 & 644.3346 & 1286.6779 & 643.8426 & L & 521.3082 & 261.1577 & 504.2817 & 252.6445 & & & 4 \\
\hline 13 & 1418.7314 & 709.8693 & 1401.7049 & 701.3561 & 1400.7209 & 700.8641 & $\mathrm{~N}$ & 408.2241 & 204.6157 & 391.1976 & 196.1024 & & & 3 \\
\hline 14 & 1565.7998 & 783.4036 & 1548.7733 & 774.8903 & 1547.7893 & 774.3983 & $F$ & 294.1812 & 147.5942 & 277.1547 & 139.0810 & & & 2 \\
\hline 15 & & & & & & & $\mathrm{~K}$ & 147.1128 & 74.0600 & 130.0863 & 65.5468 & & & 1 \\
\hline
\end{tabular}
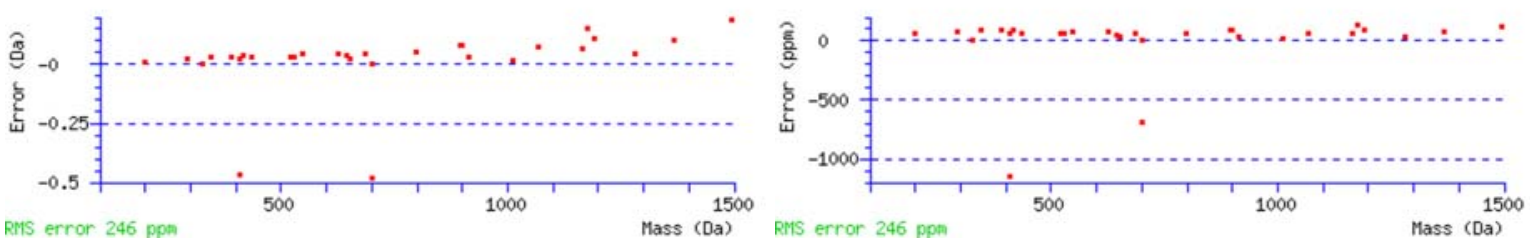

RMS error 246 ppm Mass (Da) RMS error 246 ppm Mass (Da) 


\section{Peptide View}

MS/MS Fragmentation of TTFQNLIEGFEALLK

Found in gi|22164788|ref|NP_666257.1|, Janus kinase 1 [Mus musculus]

Match to Query 584: 1722.954048 from $(862.484300,2+)$

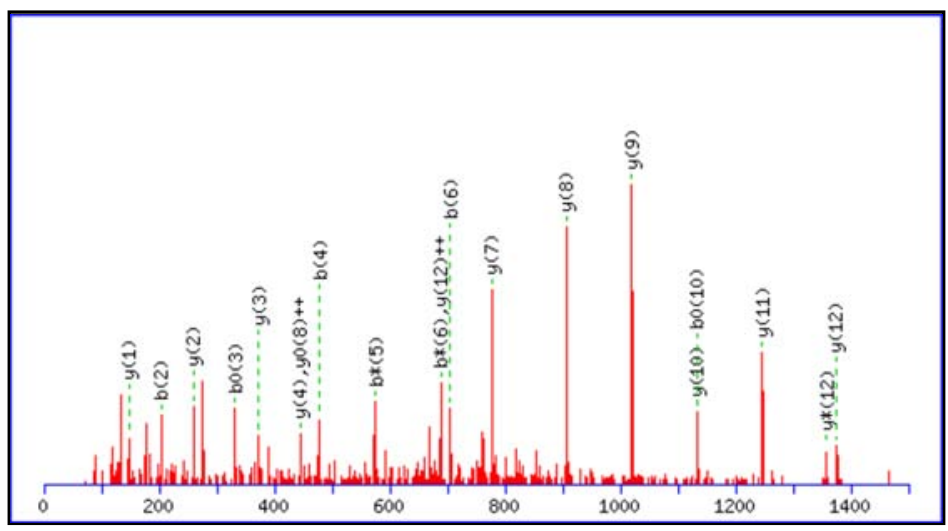

Monoisotopic mass of neutral peptide $\operatorname{Mr}$ (calc): 1722.9192

Ions Score: 72 Expect: 0.00018

Matches (Bold Red) : 20/154 fragment ions using 29 most intense peaks

\begin{tabular}{|c|c|c|c|c|c|c|c|c|c|c|c|c|c|c|}
\hline$\#$ & b & $\mathrm{b}^{++}$ & $b *$ & $b *^{++}$ & $b^{0}$ & $b^{0++}$ & Seq. & y & $y^{++}$ & y* & ++ & $y^{0}$ & $y^{0++}$ & \# \\
\hline 1 & 102.0550 & 51.5311 & & & 84.0444 & 42.5258 & $T$ & & & & & & & 15 \\
\hline 2 & 203.1026 & 102.0550 & & & 185.0921 & 93.0497 & $T$ & 162 & 811 & 1605 & 98 & 1604.8682 & \begin{tabular}{|l|}
802.9378 \\
\end{tabular} & 14 \\
\hline 3 & 350.1710 & 175.5892 & & & 332.1605 & 166.5839 & $F$ & 1521.8311 & 761.4192 & 1504.8046 & 9059 & 1503.8206 & 752.4139 & 13 \\
\hline 4 & 478.2296 & 239.6185 & 461.2031 & 231.1052 & 0.2191 & 230.6132 & $Q$ & 1374.7627 & 687.8850 & 1357.7362 & 679.3717 & 1356.7521 & 678.8797 & 12 \\
\hline 5 & 592.2726 & 296.6399 & 575.2460 & 288.1266 & 574.2620 & 287.6346 & $\mathrm{~N}$ & 1246.7041 & 623.8557 & 1229.6776 & 615.3424 & 1228.6936 & 614.8504 & 11 \\
\hline 6 & 705.3566 & 353.1819 & 688.3301 & 344.6687 & 687.3461 & 344.1767 & L & 1132 & 566.8342 & 1115 & 210 & 1114.6506 & 557.8290 & 10 \\
\hline 7 & 818.4407 & 409.7240 & 801.4141 & 401.2107 & 800.4301 & 400.7187 & I & 1019.5771 & 510.2922 & 1002.5506 & 501.7789 & 1001.5666 & 501.2869 & 9 \\
\hline 8 & 947.4833 & 474.2453 & 930.4567 & 465.7320 & 929.4727 & 465.2400 & $\mathrm{E}$ & 906.4931 & 453.7502 & 665 & 369 & 888.4825 & 444.7449 & 8 \\
\hline 9 & 1004.5047 & 502.7560 & 987.4782 & 494.2427 & 986.4942 & 493.7507 & G & 777.4505 & 389.2289 & 760.4239 & 380.7156 & 759.4399 & 380.2236 & 7 \\
\hline 10 & 1151.5731 & 576.2902 & 1134.5466 & 567.7769 & 1133.5626 & 567.2849 & $\mathrm{~F}$ & 720.4290 & 360.7182 & 703.4025 & 352.2049 & 702.4185 & 351.7129 & 6 \\
\hline 11 & 1280.6157 & 640.8115 & 1263.5892 & 632.2982 & 1262.6052 & 631.8062 & E & 573.3606 & 287.1839 & 556.3341 & 278.6707 & 555.3501 & 278.1787 & 5 \\
\hline 12 & 1351.6528 & 676.3301 & 1334.6263 & 667.8168 & 1333.6423 & 667.3248 & A & 444.3180 & 222.6627 & 915 & 214 & & & 4 \\
\hline 13 & 1464.7369 & 732.8721 & 1447.7103 & 724.3588 & 1446.7263 & 723.8668 & L & 373.2809 & 187.1441 & 356.2544 & 178.6308 & & & 3 \\
\hline 14 & 1577.8210 & 789.4141 & 1560.7944 & 780.9008 & 1559.8104 & 780.4088 & L & 260.1969 & 130.6021 & 243.1703 & 122.0888 & & & 2 \\
\hline 15 & & & & & & & $\mathrm{~K}$ & 147.1128 & 74.0600 & 130.0863 & 65.5468 & & & 1 \\
\hline
\end{tabular}
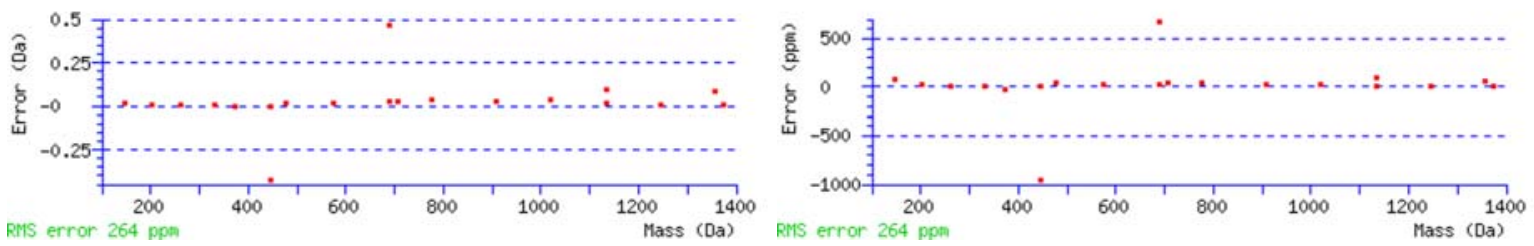


\section{Peptide View}

MS/MS Fragmentation of TVAGGAWTYNTTSAVTVK

Found in gi|6677785|ref|NP_033110.1|, ribosomal protein L37a [Mus musculus]

Match to Query 602: 1825.979048 from $\left(913.996800,2^{+}\right)$

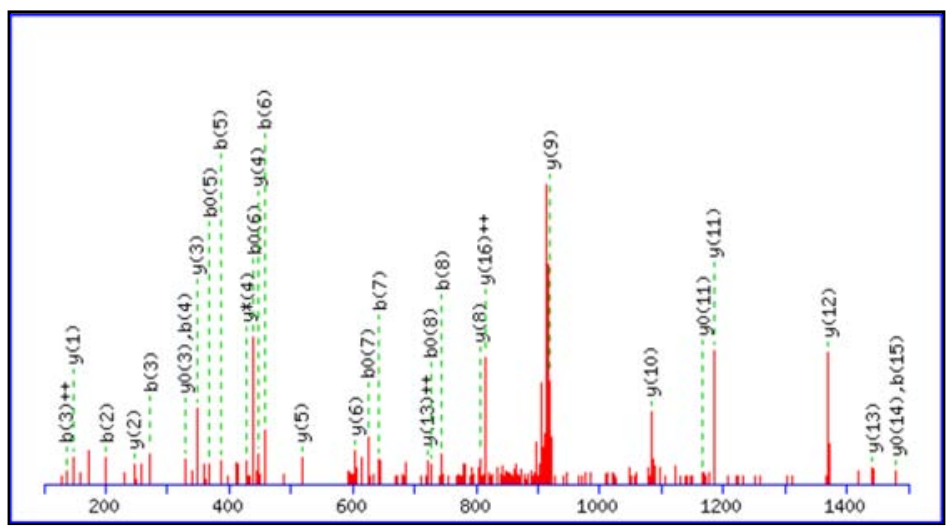

Monoisotopic mass of neutral peptide $\mathrm{Mr}$ (calc): 1825.9210

Ions Score: 55 Expect: 0.0087

Matches (Bold Red): 31/182 fragment ions using 77 most intense peaks

\begin{tabular}{|c|c|c|c|c|c|c|c|c|c|c|c|c|c|c|}
\hline \# & b & $b^{++}$ & $b *$ & $\mathrm{~b} *^{++}$ & $b^{0}$ & $b^{0++}$ & Seq. & $y$ & $y^{++}$ & y* & $y *^{++}$ & $y^{0}$ & $y^{0++}$ & $\#$ \\
\hline 1 & 102.0550 & 51.5311 & & & 84.0444 & 42.5258 & $T$ & & & & & & & 18 \\
\hline 2 & 201.1234 & 101.0653 & & & 183.1128 & 92.0600 & $\mathrm{~V}$ & 1725.8806 & 863.4439 & 1708.8541 & 854.9307 & \begin{tabular}{|l|}
1707.8700 \\
\end{tabular} & 854.4387 & 17 \\
\hline 3 & 272.1605 & 136.5839 & & & 254.1499 & 127.5786 & A & 1626.8122 & 813.9097 & 1609.7856 & 805.3965 & 1608.8016 & 804.9045 & 16 \\
\hline 4 & 329.1819 & 165.0946 & & & 311.1714 & 156.0893 & G & 1555.7751 & 778.3912 & 1538.7485 & 769.8779 & 1537.7645 & 769.3859 & 15 \\
\hline 5 & 386.2034 & 193.6053 & & & 368.1928 & 184.6001 & G & 1498.7536 & 749.8804 & 1481.7271 & 741.3672 & 1480.7431 & 740.8752 & 14 \\
\hline 6 & 457.2405 & 229.1239 & & & 439.2299 & 220.1186 & A & 1441.7322 & 721.3697 & 1424.7056 & 712.8564 & 1423.7216 & 712.3644 & 13 \\
\hline 7 & 643.3198 & 322.1635 & & & 625.3093 & 313.1583 & $w$ & 1370.6951 & 685.8512 & 1353.6685 & 677.3379 & 1352.6845 & 676.8459 & 12 \\
\hline 8 & 744.3675 & 372.6874 & & & 726.3569 & 363.6821 & $T$ & 1184.6157 & 592.8115 & 1167.5892 & 584.2982 & 1166.6052 & 583.8062 & 11 \\
\hline 9 & 907.4308 & 454.2191 & & & 889.4203 & 445.2138 & $\mathrm{Y}$ & 1083.5681 & 542.2877 & 1066.5415 & 533.7744 & 1065.5575 & 533.2824 & 10 \\
\hline 10 & 1021.4738 & 511.2405 & 1004.4472 & 272 & 1003.4632 & 502.2352 & $\mathrm{~N}$ & 920.5047 & 460.7560 & 903.4782 & 452.2427 & 902.4942 & 451.7507 & 9 \\
\hline 11 & 1122.5214 & 561.7644 & 1105.4949 & 553.2511 & 1104.5109 & 552.7591 & $T$ & 806.4618 & 403.7345 & 789.4353 & 395.2213 & 788.4512 & 394.7293 & 8 \\
\hline 12 & 1223.5691 & 612.2882 & 1206.5426 & 603.7749 & 1205.5586 & 603.2829 & $T$ & 705.4141 & 353.2107 & 688.3876 & 344.6974 & 687.4036 & 344.2054 & 7 \\
\hline 13 & 1310.6011 & 655.8042 & 1293.5746 & 647.2909 & 1292.5906 & 646.7989 & $\mathrm{~s}$ & 604.3664 & 302.6869 & 587.3399 & 294.1736 & 586.3559 & 293.6816 & 6 \\
\hline 14 & 1381.6383 & 691.3228 & 1364.6117 & 682.8095 & 1363.6277 & 682.3175 & $A$ & 517.3344 & 259.1708 & 00.3079 & 250.6576 & 499.3238 & 250.1656 & 5 \\
\hline 15 & 1480.7067 & 740.8570 & 1463.6801 & 732.3437 & 1462.6961 & 731.8517 & V & 446.2973 & 223.6523 & 429.2708 & 215.1390 & 428.2867 & 214.6470 & 4 \\
\hline 16 & 1581.7543 & 791.3808 & 1564.7278 & 782.8675 & 1563.7438 & 782.3755 & $T$ & 347.2289 & 174.1181 & 330.2023 & 165.6048 & 329.2183 & 165.1128 & 3 \\
\hline 17 & 1680.8228 & 840.9150 & 1663.7962 & 832.4017 & 1662.8122 & 831.9097 & V & 246.1812 & 123.5942 & 229.1547 & 115.0810 & & & 2 \\
\hline 18 & & & & & & & K & 147.1128 & 74.0600 & 130.0863 & 65.5468 & & & 1 \\
\hline
\end{tabular}
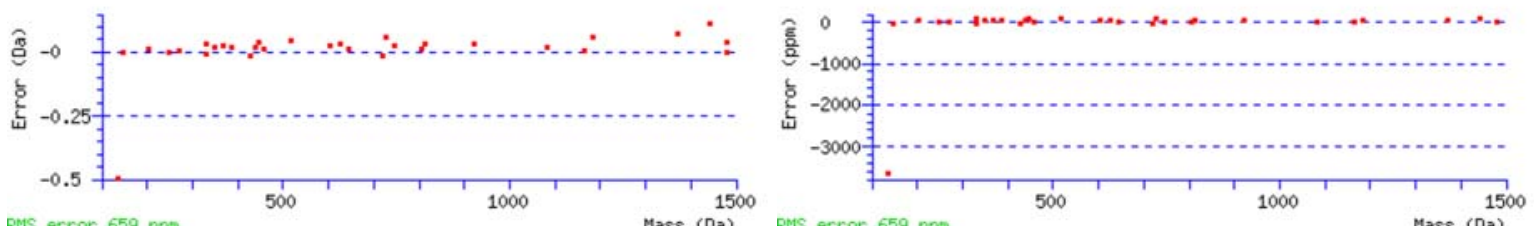


\section{Peptide View}

MS/MS Fragmentation of VIHDNFGIVEGLMTTVHAITATQK

Found in gi|41054968|ref|NP_955766.1|, glyceraldehyde-3-phosphate dehydrogenase (phosphorylating)-like [Mus musculus]

Match to Query 622: 2594.463072 from $(865.828300,3+)$

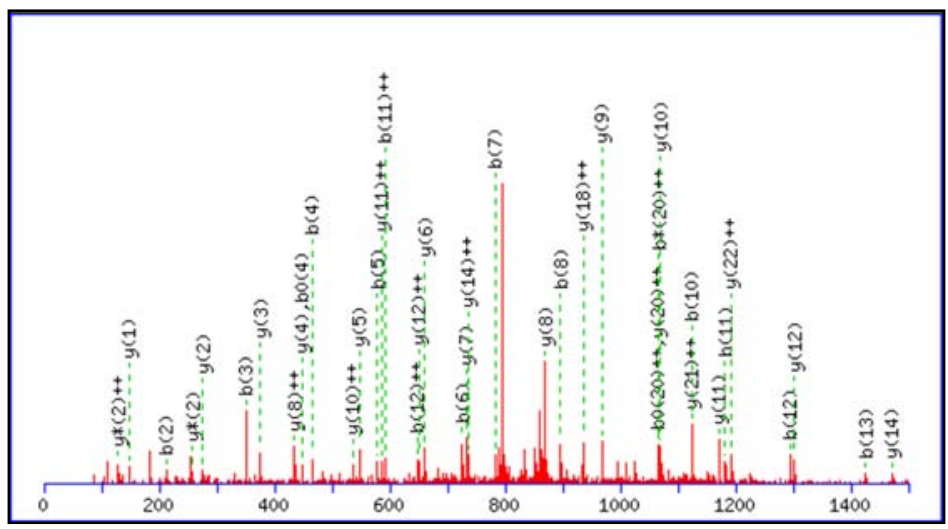

Monoisotopic mass of neutral peptide $\mathrm{Mr}$ (calc): 2594.3526

Ions Score: 98 Expect: $1.2 \mathrm{e}-006$

Matches (Bold Red): 40/258 fragment ions using 67 most intense peaks

\begin{tabular}{|c|c|c|c|c|c|c|c|c|c|c|c|c|c|c|}
\hline$\#$ & b & $\mathrm{b}^{++}$ & b* & $\mathrm{b} *^{++}$ & $b^{0}$ & $b^{0++}$ & eq. & $\mathbf{y}$ & $y^{++}$ & y* & $y *^{++}$ & $y^{0}$ & $y^{0++}$ & \# \\
\hline 1 & 100.0757 & 50.5415 & & & & & V & & & & & & & 24 \\
\hline 2 & 213.1597 & 107.0835 & & & & & I & 2915 & 1 & 49 & 61 & 2478.2809 & 123 & 23 \\
\hline 3 & 0.2187 & 5.6130 & & & & & $\mathrm{r}$ & 74 & 11 & 09 & 941 & 968 & 021 & 22 \\
\hline 4 & 465.2456 & 233.1264 & & & | & 224.1212 & D & 485 & 112 & 2229 & 646 & 2228.1379 & 726 & 21 \\
\hline 5 & 9.2885 & 0.1479 & 2.2 & 46 & 30 & 26 & $\mathrm{~N}$ & 16 & 44 & & .5511 & 2113.1110 & 591 & 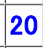 \\
\hline 6 & 726.3569 & 363.6821 & 709.3304 & 355.1688 & 708.3464 & 354.6768 & $\mathrm{~F}$ & 2017.0786 & 1009.0430 & 2000.0521 & 5297 & 1999.0681 & 377 & 19 \\
\hline 7 & 783.3784 & 392.1928 & 766.3 & 796 & 765.3678 & 876 & G & 02 & 87 & 1852 & 9955 & 1851.9997 & 035 & 18 \\
\hline 8 & 896.4625 & 448.7349 & 879.4359 & 440.2216 & 878.4519 & 439.7296 & I & 1812.9888 & 906.9980 & 1795.9622 & 898.4847 & 1794.9782 & 897.9927 & 17 \\
\hline 9 & 995.5309 & 498.2691 & 978.5043 & 489.7558 & 977.5203 & 489.2638 & V & 047 & 4560 & 1682 & .9427 & 1681.8941 & 4507 & 16 \\
\hline 0 & 1124.5735 & 562.7904 & \begin{tabular}{|l|l|}
1107.5469 \\
\end{tabular} & 554.2771 & 1106.5629 & 553.7851 & $E$ & 1600.8363 & 9218 & \begin{tabular}{|l|}
1583.8097 \\
\end{tabular} & 4085 & 1582.8257 & 65 & 15 \\
\hline 11 & 1181.5949 & 591.3011 & 1164.5684 & 582.7878 & 1163.5844 & 582.2958 & G & 1471.7937 & 736.4005 & 1454.7672 & 727.8872 & 1453.7831 & 727.3952 & 14 \\
\hline 2 & 1294.6790 & 647.8431 & \begin{tabular}{|l|}
1277.6524 \\
\end{tabular} & 639.3299 & 1276.6684 & 638.8378 & $\mathrm{~L}$ & 1414.7722 & 7.8898 & \begin{tabular}{|l|}
1397.7457 \\
\end{tabular} & 699.3765 & 1396.7617 & 8845 & 13 \\
\hline 13 & 1425.7195 & 713.3634 & 1408.6929 & 704.8501 & 1407.7089 & 704.3581 & $M$ & 1301.6882 & 651.3477 & 1284.6616 & 642.8345 & 1283.6776 & 642.3424 & 12 \\
\hline 4 & 1526.7671 & 763.8872 & 1509.7406 & 755.3739 & 1508.7566 & 754.8819 & $T$ & 1170.6477 & 75 & \begin{tabular}{|l|}
1153.6212 \\
\end{tabular} & 577.3142 & 1152.6371 & 3222 & 11 \\
\hline 15 & 1627.8148 & 814.4110 & 1610.7883 & 805.8978 & 1609.8043 & 805.4058 & $T$ & 1069.6000 & 535.3036 & 1052.5735 & 526.7904 & 1051.5895 & 526.2984 & 10 \\
\hline 6 & 1726.8832 & 863.9453 & 1709.8567 & 855.4320 & 1708.8727 & 854.9400 & $v$ & 968.5523 & 484.7798 & 951.5258 & 476.2665 & 950.5418 & 475.7745 & 9 \\
\hline 17 & 1863.9421 & 932.4747 & 1846.9156 & 923.9614 & 1845.9316 & 923.4694 & $\mathrm{H}$ & 869.4839 & 435.2456 & 852.4574 & 426.7323 & 851.4734 & 426.2403 & 8 \\
\hline 8 & 1934.9792 & 967.9933 & \begin{tabular}{|l|}
1917.9527 \\
\end{tabular} & 959.4800 & \begin{tabular}{|l|}
1916.9687 \\
\end{tabular} & 958.9880 & A & 732.4250 & 366.7161 & 715.3985 & 358.2029 & 714.4145 & 357.7109 & 7 \\
\hline 19 & 2048.0633 & 1024.5353 & 2031.0368 & 1016.0220 & 2030.0527 & 1015.5300 & I & 661.3879 & 331.1976 & 644.3614 & 322.6843 & 643.3773 & 322.1923 & 6 \\
\hline 20 & 2149.1110 & 1075.0591 & 2132.0844 & 1066.5459 & 2131.1004 & 1066.0538 & $T$ & 548.3039 & 274.6556 & 531.2773 & 266.1423 & 530.2933 & 265.6503 & 5 \\
\hline 21 & 2220.1481 & 1110.5777 & 2203.1215 & 1102.0644 & 2202.1375 & 1101.5724 & A & 447.2562 & 224.1317 & 430.2296 & 215.6184 & 429.2456 & 215.1264 & 4 \\
\hline 22 & 2321.1958 & 1161.1015 & 2304.1692 & 1152.5883 & 2303.1852 & 1152.0962 & $T$ & 376.2191 & 188.6132 & 359.1925 & 180.0999 & 358.2085 & 179.6079 & 3 \\
\hline 23 & 2449.2544 & 1225.1308 & 2432.2278 & 1216.6175 & 2431.2438 & 1216.1255 & Q & 275.1714 & 138.0893 & 258.1448 & 129.5761 & & & 2 \\
\hline 24 & & & & & & & K & 147.1128 & 4.0600 & 130.0863 & 5.5468 & & & \\
\hline
\end{tabular}
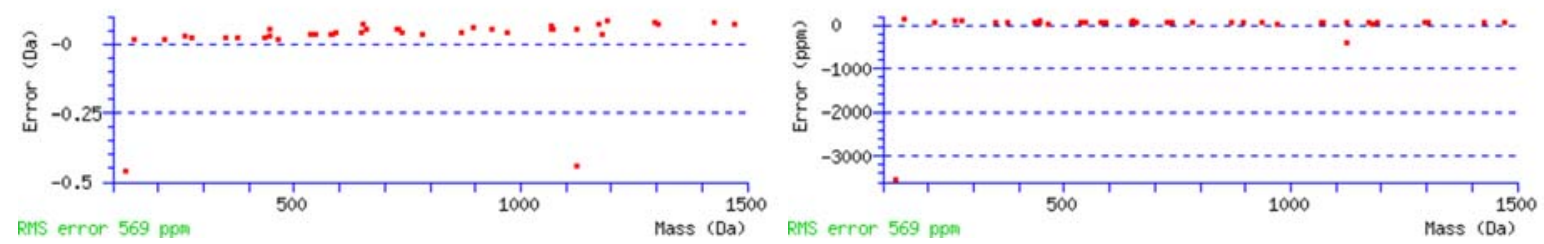


\section{Peptide View}

MS/MS Fragmentation of VINIFQSNLFQALIDIQEFYEVTLLDNPK

Found in gi|82965034|ref|XP_929204.1|, PREDICTED: similar to discs large homolog 1 isoform 6 [Mus musculus]

Match to Query 764: 3423.992172 from(1142.338000,3+)

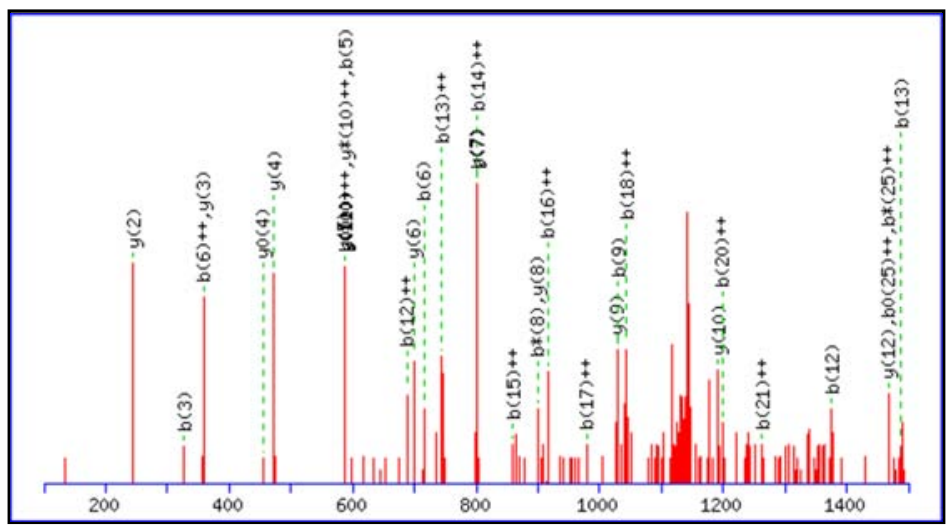

Monoisotopic mass of neutral peptide $\operatorname{Mr}(\mathrm{calc}): 3423.7965$

Ions Score: 60 Expect: 0.013

Matches (Bold Red): 34/314 fragment ions using 67 most intense peaks

\begin{tabular}{|c|c|c|c|c|c|c|c|c|c|c|c|c|c|c|}
\hline$\pi$ & b & $b^{++}$ & b* & $\mathrm{b} *^{++}$ & $b^{0}$ & $b^{0++}$ & 0 & $\mathbf{y}$ & $y^{++}$ & y* & $y *^{++}$ & $y^{0}$ & $v^{0++}$ & \# \\
\hline 1 & 100. & 415 & & & & & & & & & & & & 29 \\
\hline 2 & 213.1597 & 07.0835 & & & & & & 44 & 1 & 9 & & 19 & & 28 \\
\hline 3 & 7 & 50 & 1 & & & & & & & & & & & 2 \\
\hline $\boldsymbol{4}$ & 440.2867 & 220.6470 & 423.2 & 212. & & & & 84 & & 30 & & 79 & & 26 \\
\hline 5 & 1 & 12 & & & & & & & & & & & & 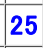 \\
\hline 6 & 715.4137 & 358.2105 & 698.3872 & 349.6972 & & & C & 2838 & 14 & 282 & 83 & 154 & & 24 \\
\hline 7 & 458 & 1.7265 & & & 784.4 & & & & & & & & & \\
\hline 8 & 916.4887 & 458.7480 & 899.4621 & 450.2347 & 898.4781 & 449. & $\mathrm{~N}$ & 2623.3654 & 131 & 2606 & 30 & 48 & $13 c$ & 22 \\
\hline 9 & 1029.5727 & 5.2900 & 12.5462 & 506.7767 & 1011.5622 & 506 & L & 224 & 1648 & 59 & 516 & 119 & & 21 \\
\hline 0 & 1176.6412 & 38.8242 & 59.6146 & 580.3109 & 1158.6306 & & $F$ & 2396.2384 & 11 & 118 & 95 & 278 & & 20 \\
\hline 1 & 1304.6997 & 2.8535 & 1287.6732 & 644.3402 & 1286.6892 & 643.8482 & $Q$ & 2249.1700 & 0886 & 2232.1434 & 5753 & 1594 & & 19 \\
\hline 2 & 1375.7368 & 8.3721 & 58.7103 & & & & A & & & & & & & 18 \\
\hline 3 & 1488.8209 & 44.9141 & 1471.7944 & 736.4008 & 1470.8103 & 088 & L & 2050.0743 & 5408 & 2033.0477 & 0275 & 0637 & 101 & 17 \\
\hline 4 & 01.9050 & 1.4561 & 1584. & 792.9428 & 158 & & & 902 & & & & & & \\
\hline 5 & 716.9319 & 858.9696 & 1699.9054 & 850.4563 & 1698.9213 & 849 & D & 3061 & 912.4567 & 1806 & 34 & 3956 & 14 & 15 \\
\hline 6 & 30.0160 & 16 & 94 & & & & & 92 & & & & & & \\
\hline 7 & 958.0745 & 979.5409 & 1941.0480 & 971.0276 & 1940.0640 & 970 & $Q$ & 159 & 798.4012 & 157 & 79 & 846 & & 13 \\
\hline 8 & 087.1171 & 1044.0622 & 2070.0906 & 035.5489 & 2069.1066 & 69 & & 1467 & & & 8586 & 260 & & 12 \\
\hline 9 & 2234.1855 & 1117.5964 & 2217.1590 & 1109.0831 & 2216.1750 & 1108 & $\mathrm{~F}$ & 40 & 506 & 74 & 3373 & 334 & & 11 \\
\hline 20 & 89 & 1199 & 238 & 1190.6148 & 2379.2383 & 1190 & Y & 1191.6256 & 596.3164 & 990 & 587.8031 & 1173.6150 & 587 & 10 \\
\hline 21 & 2526.2915 & 1263.6494 & 2509.2649 & 1255.1361 & 809 & 1254.6441 & $E$ & 1028.5622 & 514.7848 & 1011.5357 & 506.2715 & 5517 & 7795 & 9 \\
\hline 22 & 2625.3599 & 13.1836 & 2608.3333 & 1304.6703 & 2607.3493 & 1304.1783 & $\mathrm{~V}$ & 899.5196 & 50.2635 & 82.4931 & 41.7502 & 381.5091 & 41.2582 & 8 \\
\hline 23 & 2726.4076 & 63.7074 & 2709.3810 & 1355.1941 & 2708.3970 & 1354.7021 & $T$ & 00.4512 & 400.7293 & 783.4247 & 392.2160 & 82.4407 & 391.7240 & 7 \\
\hline 24 & 2839.4916 & 20.2494 & 2822.4651 & 1411.7362 & 2821.4811 & 1411.2442 & $\mathrm{~L}$ & 99.4036 & 350.2054 & 82.3770 & 341.6921 & 81.3930 & 01 & 6 \\
\hline 25 & \begin{tabular}{|l|}
2952.5757 \\
\end{tabular} & 76.7915 & 2935.5491 & 1468.2782 & 2934.5651 & 146 & L & 586.3195 & 293.6634 & 569.2929 & 285.1501 & 568.3089 & 284.6581 & 5 \\
\hline 26 & 3067.6026 & 334.3049 & 3050.5761 & 1525.7917 & 3049.5921 & 1525.2997 & 0 & 173.2354 & 237.1214 & 456.2089 & 228.6081 & 455.2249 & 228.1161 & 4 \\
\hline 27 & 3181.6 & 64 & 3164 & 1582. & 3163 & 1582 & 10 & 358.2085 & & & & & & 3 \\
\hline 28 & 278.6983 & 39.8528 & 3261.6718 & 1631.3395 & 3260.6877 & 1630.8475 & $P$ & 244.1656 & 122.5864 & 227.1390 & 114.0731 & & & 2 \\
\hline 29 & & & & & & & $\mathrm{~K}$ & 147.1128 & 74.0600 & 130.0863 & 65.5468 & & & 1 \\
\hline
\end{tabular}
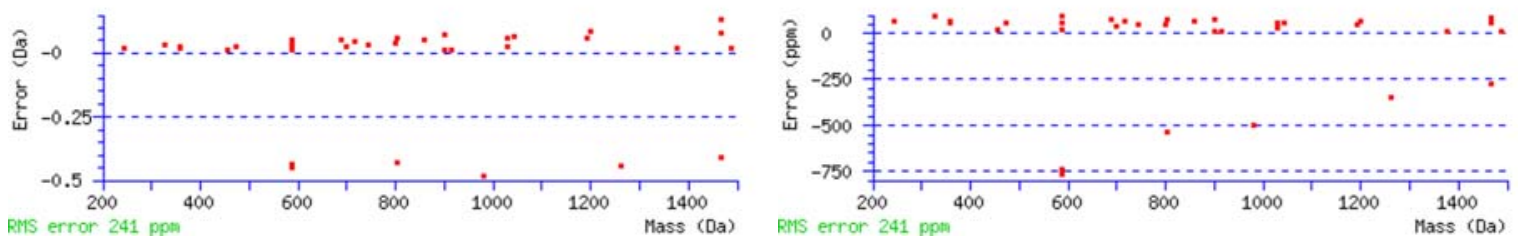


\section{Peptide View}

MS/MS Fragmentation of VLALIQAWADAFR

Found in gi|84875524|ref|NP_001034181.1|, target of myb1-like 2 isoform b [Mus musculus]

Match to Query 307: 1472.888048 from $(737.451300,2+)$

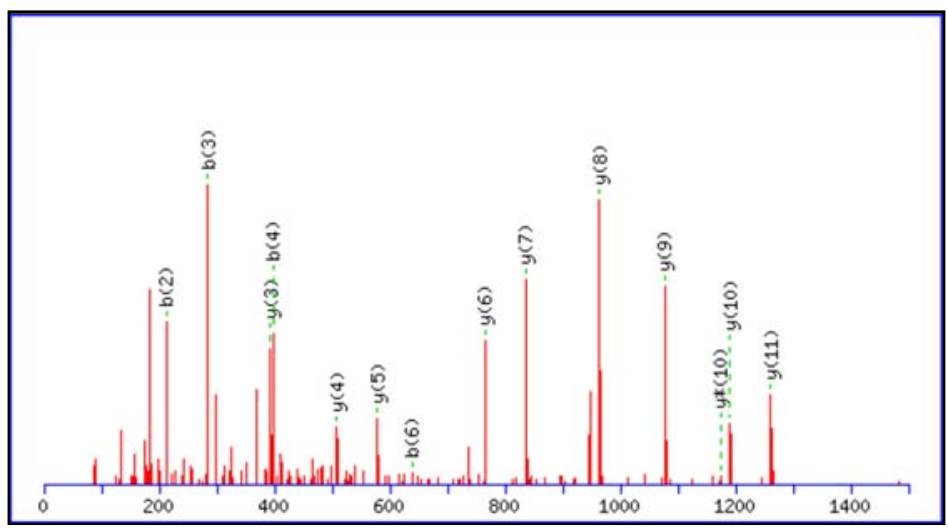

Monoisotopic mass of neutral peptide $\operatorname{Mr}$ (calc): 1472.8139

Ions Score: 63 Expect: 0.00093

Matches (Bold Red): 14/110 fragment ions using 28 most intense peaks

\begin{tabular}{|c|c|c|c|c|c|c|c|c|c|c|c|c|c|c|}
\hline \# & b & $\mathrm{b}^{++}$ & b* & $b *^{++}$ & $b^{0}$ & $b^{0++}$ & Seq. & $y$ & $y^{++}$ & y* & $y *^{++}$ & $y^{0}$ & $y^{0++}$ & $\#$ \\
\hline 1 & 100.0757 & 50.5415 & & & & & V & & & & & & & 13 \\
\hline 2 & 213.1597 & 107.0835 & & & & & L & 1374.7528 & 687.8800 & 1357.7263 & 679.3668 & 1356.7422 & 678.8748 & 12 \\
\hline 3 & 284.1969 & 142.6021 & & & & & $A$ & 1261.6688 & 631.3380 & 1244.6422 & 622.8247 & 1243.6582 & 622.3327 & 11 \\
\hline 4 & 397.2809 & 199.1441 & & & & & L & 1190.6316 & 595.8195 & 1173.6051 & 587.3062 & 1172.6211 & 586.8142 & 10 \\
\hline 5 & 510.3650 & 255.6861 & & & & & I & 1077.5476 & 539.2774 & 1060.5210 & 530.7642 & 1059.5370 & 530.2721 & 9 \\
\hline 6 & 638.4236 & 319.7154 & 621.3970 & 311.2021 & & & $Q$ & 964.4635 & 482.7354 & 947.4370 & 474.2221 & 946.4530 & 473.7301 & 8 \\
\hline 7 & 709.4607 & 355.2340 & 692.4341 & 346.7207 & & & A & 836.4049 & 418.7061 & 819.3784 & 410.1928 & 818.3944 & 409.7008 & 7 \\
\hline 8 & 895.5400 & 448.2736 & 878.5134 & 439.7604 & & & W & 765.3678 & 383.1876 & 748.3413 & 374.6743 & 747.3573 & 374.1823 & 6 \\
\hline 9 & 966.5771 & 483.7922 & 949.5505 & 475.2789 & & & A & 579.2885 & 290.1479 & 562.2620 & 281.6346 & 561.2780 & 281.1426 & 5 \\
\hline \begin{tabular}{l|l}
10 & 1 \\
\end{tabular} & 1081.6040 & 541.3057 & 1064.5775 & 532.7924 & 1063.5935 & 532.3004 & D & 508.2514 & 254.6293 & 491.2249 & 246.1161 & 490.2408 & 245.6241 & 4 \\
\hline \begin{tabular}{l|l}
11 & 1
\end{tabular} & 1152.6411 & 576.8242 & 1135.6146 & 568.3109 & 1134.6306 & 567.8189 & A & 393.2245 & 197.1159 & 376.1979 & 188.6026 & & & 3 \\
\hline \begin{tabular}{l|l}
12 & 1
\end{tabular} & 1299.7095 & 650.3584 & 1282.6830 & 641.8451 & 1281.6990 & 641.3531 & $F$ & 322.1874 & 161.5973 & 305.1608 & 153.0840 & & & 2 \\
\hline 13 & & & & & & & $\mathrm{R}$ & 175.1190 & 88.0631 & 158.0924 & 79.5498 & & & 1 \\
\hline
\end{tabular}
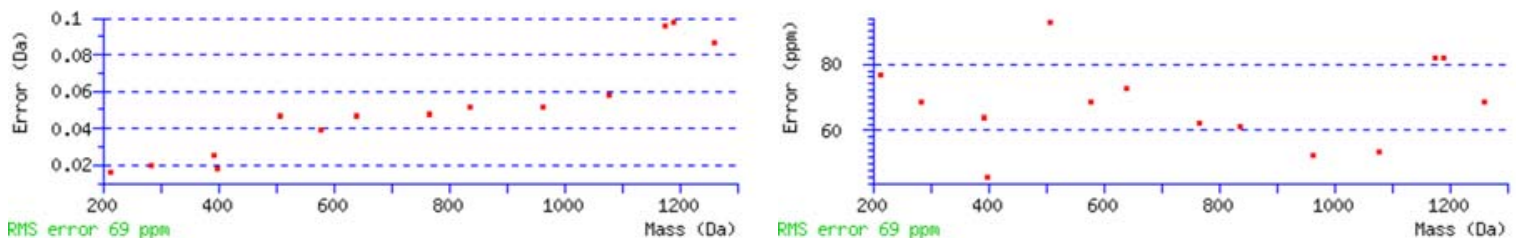


\section{Peptide View}

MS/MS Fragmentation of VLNLIQSWADAFR

Found in gi|94378129|ref|XP_001003823.1|, PREDICTED: similar to Target of Myb protein 1 isoform 6 [Mus musculus]

Match to Query 481: 1531.844048 from $\left(766.929300,2^{+}\right)$

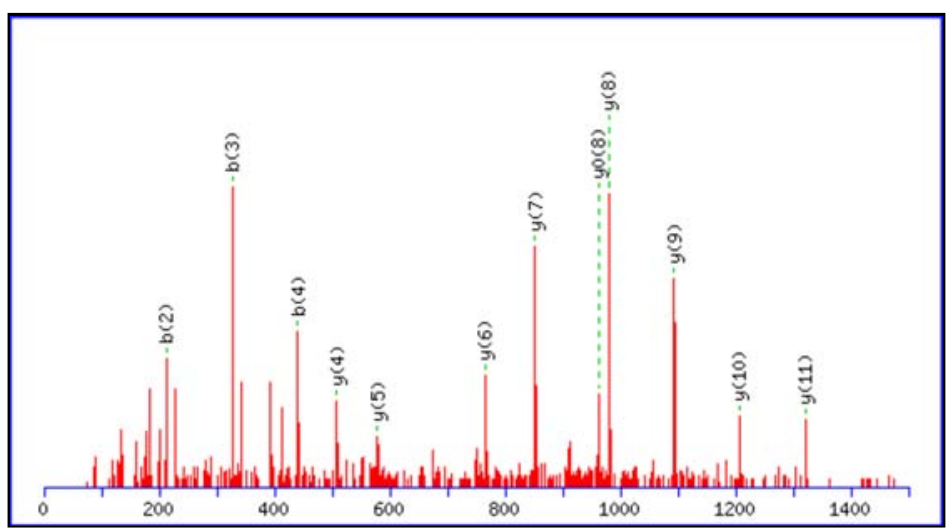

Monoisotopic mass of neutral peptide $\mathrm{Mr}$ (calc): 1531.8147

Ions Score: 71 Expect: 0.00014

Matches (Bold Red): 12/122 fragment ions using 19 most intense peaks

\begin{tabular}{|c|c|c|c|c|c|c|c|c|c|c|c|c|c|c|}
\hline \# & b & $b^{++}$ & b* & $b *^{++}$ & $b^{0}$ & $b^{0++}$ & Seq. & $y$ & $\mathrm{y}^{++}$ & $\mathrm{y} *$ & $\mathrm{y*}^{++}$ & $y^{0}$ & $y^{0++}$ & $\#$ \\
\hline 1 & 100.0757 & 50.5415 & & & & & V & & & & & & & 13 \\
\hline 2 & 213.1597 & 107.0835 & & & & & L & 1433.7536 & 717.3804 & $4 \quad 1416.7270$ & 708.8671 & 1415.7430 & 708.3751 & 112 \\
\hline 3 & 327.2027 & 164.1050 & 310.1761 & 155.5917 & & & $\mathrm{~N}$ & 1320.6695 & 660.8384 & \begin{tabular}{l|l}
4 & 1303.6429
\end{tabular} & 652.3251 & 1302.6589 & 651.8331 & \begin{tabular}{l|l}
11 & 11
\end{tabular} \\
\hline 4 & 440.2867 & 220.6470 & 423.2602 & 212.1337 & & & L & 1206.6266 & 603.8169 & 1189.6000 & 595.3036 & 1188.6160 & 594.8116 & 610 \\
\hline 5 & 553.3708 & 277.1890 & 536.3442 & 268.6758 & & & I & 1093.5425 & 547.2749 & 91076.5160 & 538.7616 & 1075.5319 & 538.2696 & \begin{tabular}{l|l}
6 & 9
\end{tabular} \\
\hline 6 & 681.4294 & 341.2183 & 664.4028 & 332.7051 & & & $\mathbf{Q}$ & 980.4584 & 490.7329 & 9963.4319 & 482.2196 & 962.4479 & 481.7276 & 8 \\
\hline 7 & 768.4614 & 384.7343 & 751.4349 & 376.2211 & 750.4508 & 375.7291 & $S$ & 852.3999 & 426.7036 & 835.3733 & 418.1903 & 834.3893 & 417.6983 & 37 \\
\hline 8 & 954.5407 & 477.7740 & 937.5142 & 469.2607 & 936.5302 & 468.7687 & w & 765.3678 & 383.1876 & 748.3413 & 374.6743 & 747.3573 & 374.1823 & $\begin{array}{ll}3 & 6\end{array}$ \\
\hline 9 & 1025.5778 & 513.2926 & 1008.5513 & 504.7793 & 1007.5673 & 504.2873 & A & 579.2885 & 290.1479 & 562.2620 & 281.6346 & 561.2780 & 281.1426 & 65 \\
\hline 10 & 1140.6048 & 570.8060 & 1123.5782 & 562.2927 & 1122.5942 & 561.8007 & D & 508.2514 & 254.6293 & 491.2249 & 246.1161 & 490.2408 & 245.6241 & 4 \\
\hline 11 & 1211.6419 & 606.3246 & 1194.6153 & 597.8113 & 1193.6313 & 597.3193 & A & 393.2245 & 197.1159 & 376.1979 & 188.6026 & & & 3 \\
\hline 12 & 1358.7103 & 679.8588 & 1341.6837 & 671.3455 & 1340.6997 & 670.8535 & $F$ & 322.1874 & 161.5973 & 305.1608 & 153.0840 & & & 2 \\
\hline 13 & & & & & & & $\mathbf{R}$ & 175.1190 & 88.0631 & 158.0924 & 79.5498 & & & 1 \\
\hline
\end{tabular}
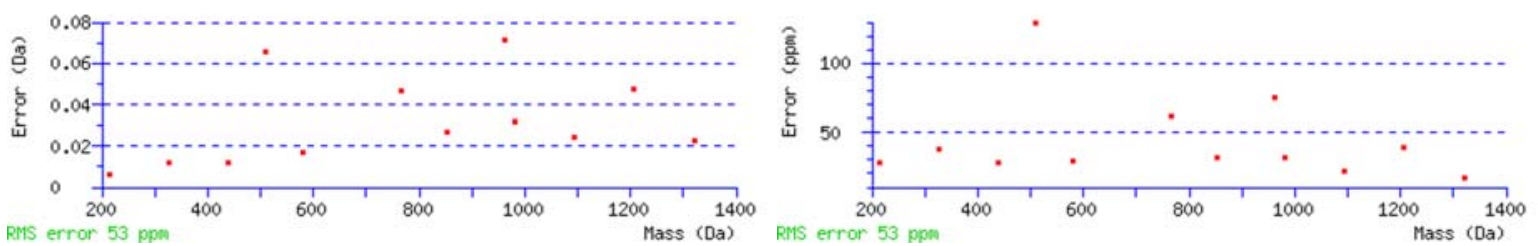


\section{Peptide View}

MS/MS Fragmentation of VTLYDPMSGILTSVQTK

Found in gi|56699423|ref|NP_035020.2|, neural precursor cell expressed, developmentally down-regulated gene 4 [Mus musculus]

Match to Query 624: 1851.970848 from $\left(926.992700,2^{+}\right)$

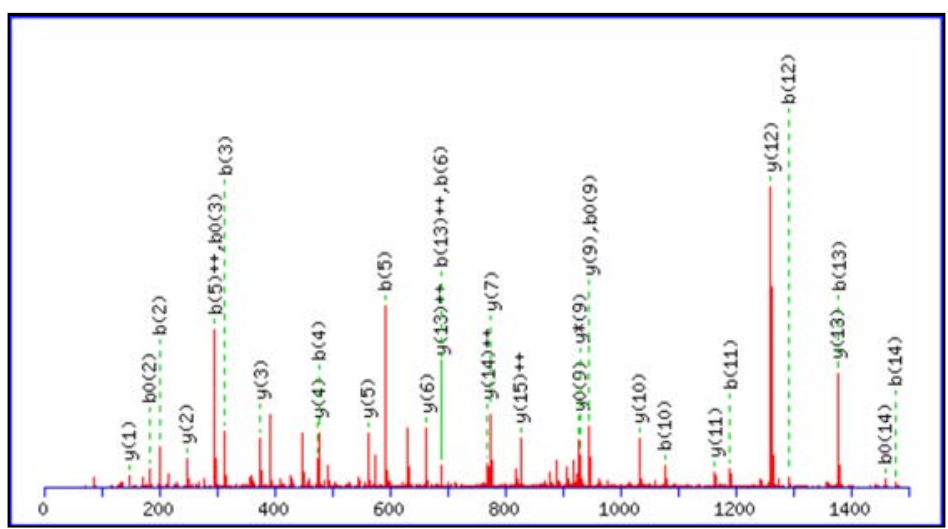

Monoisotopic mass of neutral peptide $\mathrm{Mr}$ (calc): 1851.9652

Ions Score: 85 Expect: 1e-005

Matches (Bold Red): 33/160 fragment ions using 53 most intense peaks

\begin{tabular}{|c|c|c|c|c|c|c|c|c|c|c|c|c|c|c|}
\hline$\#$ & b & $b^{++}$ & $b *$ & $b *^{++}$ & $b^{0}$ & $b^{0++}$ & Seq. & y & $y^{++}$ & y* & $y *^{++}$ & $y^{0}$ & $y^{0++}$ & $\#$ \\
\hline 1 & 100.0757 & 50.5415 & & & & & V & & & & & & & 17 \\
\hline 2 & 201.1234 & 101.0653 & & & 183.1128 & 92.0600 & $T$ & 1753.9040 & 877.4557 & 1736.8775 & 868.9424 & 1735.8935 & 868.4504 & 16 \\
\hline 3 & 314.2074 & 157.6074 & & & 296.1969 & 148.6021 & L & 1652.8564 & 826.9318 & 1635.8298 & 818.4185 & 1634.8458 & 817.9265 & 15 \\
\hline 4 & 477.2708 & 239.1390 & & & 459.2602 & 230.1337 & $Y$ & 1539.7723 & 770.3898 & 1522.7458 & 761.8765 & 1521.7617 & 761.3845 & 14 \\
\hline 5 & 592.2977 & 296.6525 & & & 574.2871 & 287.6472 & D & 1376.7090 & 688.8581 & 1359.6824 & 680.3448 & 1358.6984 & 679.8528 & 13 \\
\hline 6 & 689.3505 & 345.1789 & & & 671.3399 & 336.1736 & $P$ & 1261.6820 & 631.3447 & 1244.6555 & 622.8314 & 1243.6715 & 622.3394 & 12 \\
\hline 7 & 820.3909 & |410.6991 & & & 802.3804 & 401.6938 & M & 1164.6293 & 582.8183 & 1147.6027 & 574.3050 & 1146.6187 & 573.8130 & 11 \\
\hline 8 & 907.4230 & 454.2151 & & & 889.4124 & 445.2098 & $S$ & 1033.5888 & 517.2980 & 1016.5622 & 508.7848 & 1015.5782 & 508.2928 & 10 \\
\hline 9 & 964.4444 & 482.7259 & & & 946.4339 & 473.7206 & G & 946.5568 & 473.7820 & 929.5302 & 465.2687 & 928.5462 & 464.7767 & 9 \\
\hline 10 & 1077.5285 & 539.2679 & & & 1059.5179 & 530.2626 & I & 889.5353 & 445.2713 & 872.5088 & 436.7580 & 871.5247 & 436.2660 & 8 \\
\hline 11 & 1190.6125 & 595.8099 & & & 1172.6020 & 586.8046 & L & 776.4512 & 388.7293 & 759.4247 & 380.2160 & 758.4407 & 379.7240 & 7 \\
\hline 12 & 1291.6602 & 646.3338 & & & 1273.6497 & 637.3285 & $T$ & 663.3672 & 332.1872 & 646.3406 & 323.6740 & 645.3566 & 323.1819 & 6 \\
\hline 13 & 1378.6923 & 689.8498 & & & 1360.6817 & 680.8445 & $S$ & 562.3195 & 281.6634 & 545.2930 & 273.1501 & 544.3089 & 272.6581 & 5 \\
\hline 14 & 1477.7607 & 739.3840 & & & 1459.7501 & 730.3787 & V & 475.2875 & 238.1474 & 458.2609 & 229.6341 & 457.2769 & 229.1421 & 4 \\
\hline 15 & 1605.8192 & 803.4133 & 1588.7927 & 794.9000 & 1587.8087 & 794.4080 & $Q$ & 376.2191 & 188.6132 & 359.1925 & 180.0999 & 358.2085 & 179.6079 & 3 \\
\hline 16 & 1706.8669 & 853.9371 & 1689.8404 & 845.4238 & 1688.8564 & 844.9318 & $T$ & 248.1605 & 124.5839 & 231.1339 & 116.0706 & 230.1499 & 115.5786 & 2 \\
\hline 17 & & & & & & & $\mathrm{~K}$ & 147.1128 & 74.0600 & 130.0863 & 65.5468 & & & - \\
\hline
\end{tabular}
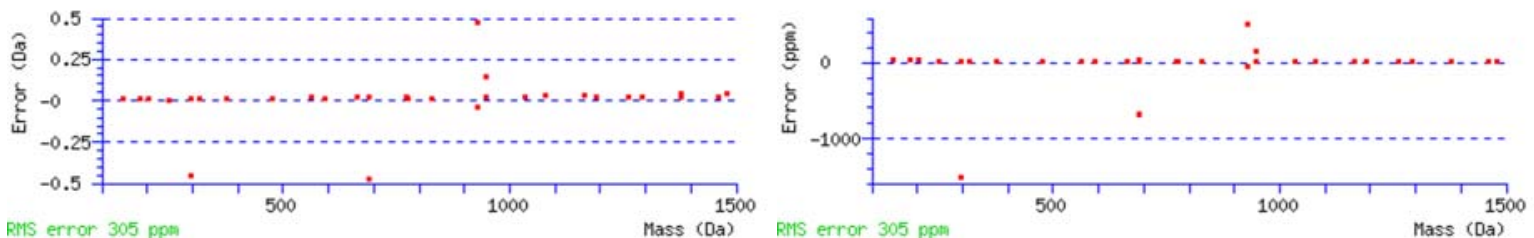


\section{Peptide View}

MS/MS Fragmentation of YGFSDPLTFNSVVELINHYR

Found in gi|33468921|ref|NP_035215.1|, phosphatidylinositol 3-kinase, regulatory subunit, polypeptide 1 isoform b [Mus musculus]

Match to Query 822: 2370.364872 from(791.128900,3+)

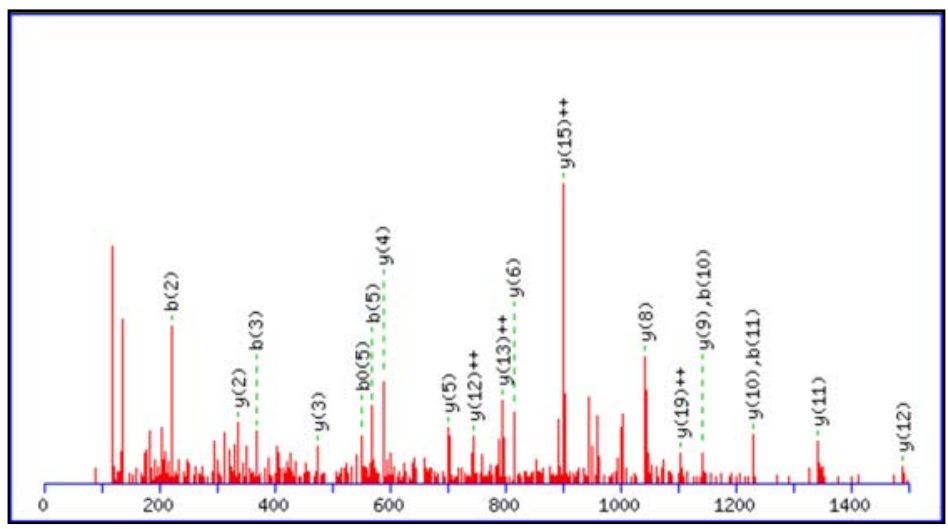

Monoisotopic mass of neutral peptide $\operatorname{Mr}(\mathrm{calc}): 2370.1644$

Ions Score: 58 Expect: 0.0096

Matches (Bold Red): 20/192 fragment ions using 34 most intense peaks

\begin{tabular}{|c|c|c|c|c|c|c|c|c|c|c|c|c|c|c|}
\hline$\#$ & b & $b^{++}$ & b* & $b *^{++}$ & $b^{0}$ & $b^{0++}$ & Seq. & y & $\mathrm{y}^{++}$ & y* & $y *^{++}$ & $y^{0}$ & $y^{0++}$ & $\#$ \\
\hline 1 & 164.0706 & 82.5389 & & & & & $Y$ & & & & & & & 20 \\
\hline 2 & 221.0921 & 111.0497 & & & & & G & 2208.1084 & 1104.5578 & 2191.0818 & 1096.0445 & 2190.0978 & 1095.5525 & 19 \\
\hline 3 & 368.1605 & 184.5839 & & & & & $\mathrm{~F}$ & 2151.0869 & 1076.0471 & 2134.0604 & 1067.5338 & 2133.0763 & 1067.0418 & 18 \\
\hline 4 & 455.1925 & 228.0999 & & & 437.1819 & 219.0946 & $S$ & 2004.0185 & 1002.5129 & 1986.9919 & 993.9996 & 1986.0079 & 993.5076 & 17 \\
\hline 5 & 570.2194 & 285.6134 & & & 552.2089 & 276.6081 & D & 1916.9865 & 958.9969 & 1899.9599 & 950.4836 & 1898.9759 & 949.9916 & 16 \\
\hline 6 & 667.2722 & 334.1397 & & & 649.2616 & 325.1345 & $P$ & 1801.9595 & 901.4834 & 1784.9330 & 892.9701 & 1783.9490 & 892.4781 & 15 \\
\hline 7 & 780.3563 & 390.6818 & & & 762.3457 & 381.6765 & L & 1704.9068 & 852.9570 & 1687.8802 & 844.4437 & 1686.8962 & 843.9517 & 14 \\
\hline 8 & 881.4039 & 441.2056 & & & 863.3934 & 432.2003 & $\mathrm{~T}$ & 1591.8227 & 796.4150 & 1574.7962 & 787.9017 & 1573.8121 & 787.4097 & 13 \\
\hline 9 & 1028.4724 & 514.7398 & & & 1010.4618 & 505.7345 & $F$ & 1490.7750 & 745.8911 & 1473.7485 & 737.3779 & 1472.7645 & 736.8859 & 12 \\
\hline 10 & 1142.5153 & 571.7613 & 1125.4887 & 563.2480 & 1124.5047 & 562.7560 & $\mathrm{~N}$ & 1343.7066 & 672.3569 & 1326.6801 & 663.8437 & 1325.6960 & 663.3517 & 11 \\
\hline 11 & 1229.5473 & 615.2773 & 1212.5208 & 606.7640 & 1211.5368 & 606.2720 & s & 1229.6637 & 615.3355 & 1212.6371 & 606.8222 & 1211.6531 & 606.3302 & 10 \\
\hline 12 & 1328.6157 & 664.8115 & 1311.5892 & 656.2982 & 1310.6052 & 655.8062 & V & 1142.6317 & 571.8195 & 1125.6051 & 563.3062 & 1124.6211 & 562.8142 & 9 \\
\hline 13 & 1427.6841 & 714.3457 & 1410.6576 & 705.8324 & 1409.6736 & 705.3404 & V & 1043.5632 & 522.2853 & 1026.5367 & 513.7720 & 1025.5527 & 513.2800 & 8 \\
\hline 14 & 1556.7267 & 778.8670 & 1539.7002 & 770.3537 & 1538.7162 & 769.8617 & $E$ & 944.4948 & 472.7511 & 927.4683 & 464.2378 & 926.4843 & 463.7458 & 7 \\
\hline 15 & 1669.8108 & 835.4090 & 1652.7842 & 826.8958 & 1651.8002 & 826.4037 & $L$ & 815.4522 & 408.2298 & 798.4257 & 399.7165 & & & 6 \\
\hline 16 & \begin{tabular}{|l|}
1782.8948 \\
\end{tabular} & 891.9511 & 1765.8683 & 883.4378 & 1764.8843 & 882.9458 & I & 702.3682 & 351.6877 & 685.3416 & 343.1745 & & & 5 \\
\hline 17 & 1896.9378 & 948.9725 & 1879.9112 & 940.4593 & 1878.9272 & 939.9672 & $\mathrm{~N}$ & 589.2841 & 295.1457 & 572.2576 & 286.6324 & & & 4 \\
\hline 18 & 2033.9967 & 1017.5020 & 2016.9701 & 1008.9887 & 2015.9861 & 1008.4967 & $\mathrm{H}$ & 475.2412 & 238.1242 & 458.2146 & 229.6110 & & & 3 \\
\hline 19 & 2197.0600 & $\mid 1099.0336$ & 2180.0335 & 1090.5204 & 2179.0495 & 1090.0284 & $Y$ & 338.1823 & 169.5948 & 321.1557 & 161.0815 & & & 2 \\
\hline 20 & & & & & & & $\mathrm{R}$ & 175.1190 & 88.0631 & 158.0924 & 79.5498 & & & 1 \\
\hline
\end{tabular}
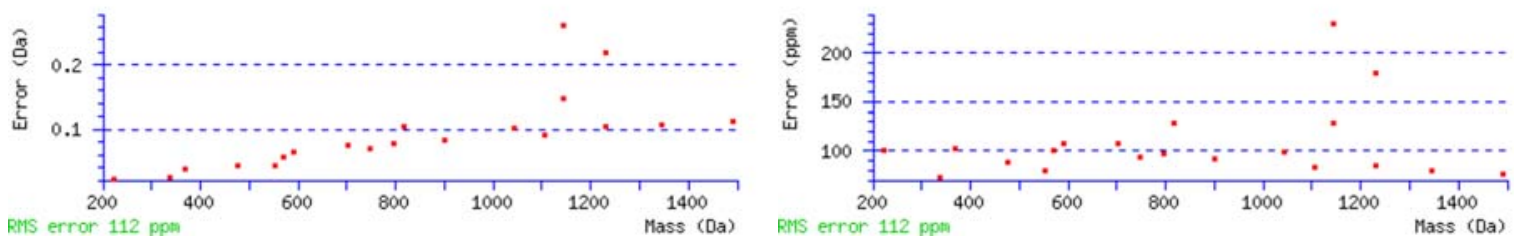


\section{Peptide View}

MS/MS Fragmentation of YMDDLQLVIEVFQK

Found in gi|46560563|ref|NP_035495.2|, SH3 domain protein 1B [Mus musculus]

Match to Query 416: 1739.943048 from(870.978800,2+)

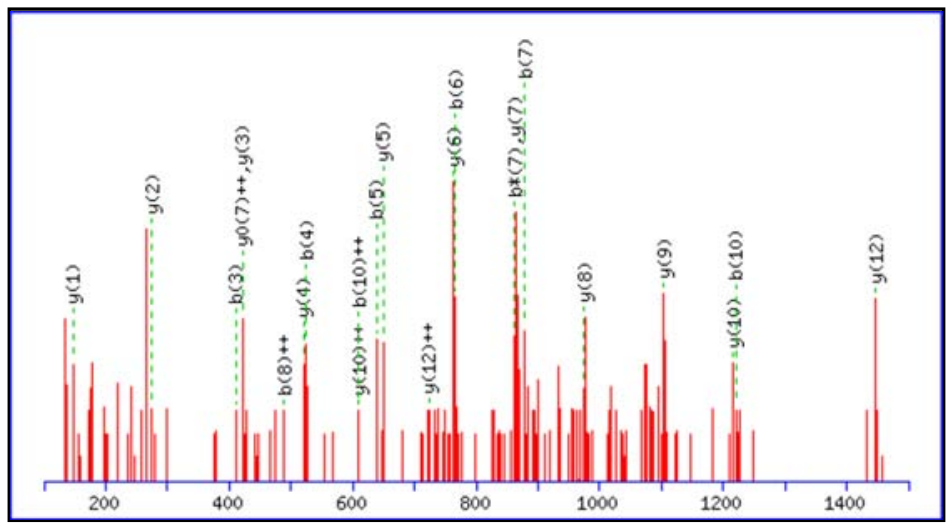

Monoisotopic mass of neutral peptide $\mathrm{Mr}$ (calc): 1739. 8804

Ions Score: 50 Expect: 0.025

Matches (Bold Red): 23/134 fragment ions using 53 most intense peaks

\begin{tabular}{|c|c|c|c|c|c|c|c|c|c|c|c|c|c|c|}
\hline \# & b & $b^{++}$ & $b *$ & $b *^{++}$ & $b^{0}$ & $b^{0++}$ & Seq. & y & $y^{++}$ & y* & $y *^{++}$ & $y^{0}$ & $y^{0++}$ & $\#$ \\
\hline 1 & 164.0706 & 82.5389 & & & & & $\mathrm{Y}$ & & & & & & & 14 \\
\hline 2 & 295.1111 & 148.0592 & & & & & $M$ & 577.8243 & 789.4158 & 1560.7978 & 780.9025 & 1559.8138 & 780.4105 & 13 \\
\hline 3 & 410.1380 & 205.5727 & & & 392.1275 & 196.5674 & D & 1446.7838 & 723.8956 & 1429.7573 & 715.3823 & 1428.7733 & 714.8903 & 12 \\
\hline 4 & 5.1650 & 263.0861 & & & 507.1544 & 254.0808 & D & 1331.7569 & 666.3821 & 1314.7304 & 657.8688 & 1313.7463 & 657.3768 & 11 \\
\hline 5 & 638.2490 & 319.6282 & & & 620.2385 & 310.6229 & L & 1216.7300 & 608.8686 & 1199.7034 & 600.3553 & 1198.7194 & 599.8633 & 10 \\
\hline 6 & 766.3076 & 383.6574 & 49.2811 & 42 & 748.2970 & 374.6522 & $Q$ & 1103.6459 & 552.3266 & 1086.6194 & 543.8133 & 1085.6353 & 543.3213 & 9 \\
\hline 7 & 879.3917 & 440.1995 & 862.3651 & 431.6862 & 861.3811 & 431.1942 & $L$ & 975.5873 & 488.2973 & 958.5608 & 479.7840 & 957.5768 & 479.2920 & 8 \\
\hline 8 & 978.4601 & 489.7337 & 61.4335 & 481.2204 & 960.4495 & 480.7284 & V & 862.5033 & 431.7553 & 845.4767 & 423.2420 & 844.4927 & 422.7500 & 7 \\
\hline 9 & 1091.5441 & 546.2757 & 1074.5176 & 537.7624 & 1073.5336 & 537.2704 & I & 763.4349 & 382.2211 & 083 & 373.7078 & 745.4243 & 373.2158 & 6 \\
\hline 101 & 1220.5867 & 610.7970 & 1203.5602 & 602.2837 & 1202.5762 & 601.7917 & $E$ & 650.3508 & 325.6790 & 633.3242 & 317.1658 & 632.3402 & 316.6738 & 5 \\
\hline 11 & 1319.6551 & 660.3312 & 1302.6286 & 651.8179 & 1301.6446 & 651.3259 & V & 521.3082 & 261.1577 & 504.2817 & 252.6445 & & & 4 \\
\hline 12 & 1466.7235 & 733.8654 & 1449.6970 & 725.3521 & 1448.7130 & 724.8601 & $\mathrm{~F}$ & 422.2398 & 211.6235 & 405.2132 & 203.1103 & & & 3 \\
\hline 13 & 1594.7821 & |797.8947 & 1577.7556 & 789.3814 & 1576.7716 & 788.8894 & $Q$ & 275.1714 & 138.0893 & 258.1448 & 129.5761 & & & 2 \\
\hline 14 & & & & & & & $K$ & 147.1128 & 74.0600 & 130.0863 & 65.5468 & & & 1 \\
\hline
\end{tabular}
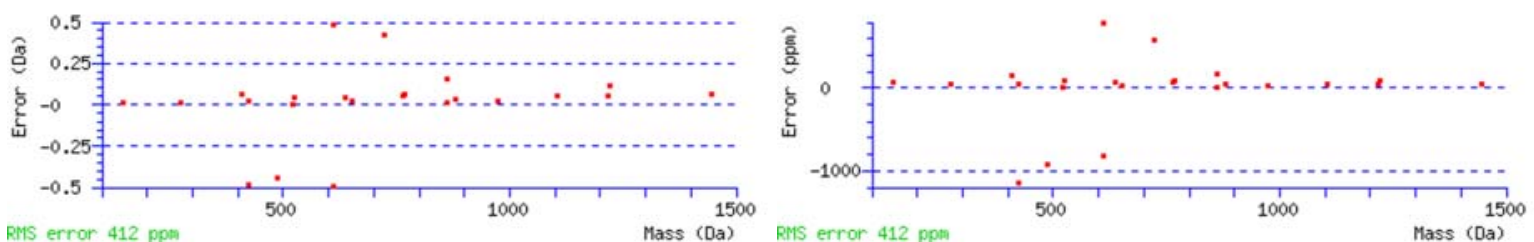

RMS error 412 ppm Mass (Da) RMS error 412 ppm Mass (Da) 


\section{Peptide View}

MS/MS Fragmentation of YTAVGQLVQDLLTQVR

Found in gi|21703974|ref|NP_663470.1|, Ras and Rab interactor 1 [Mus musculus]

Match to Query 1016: 1803.110648 from $(902.562600,2+)$

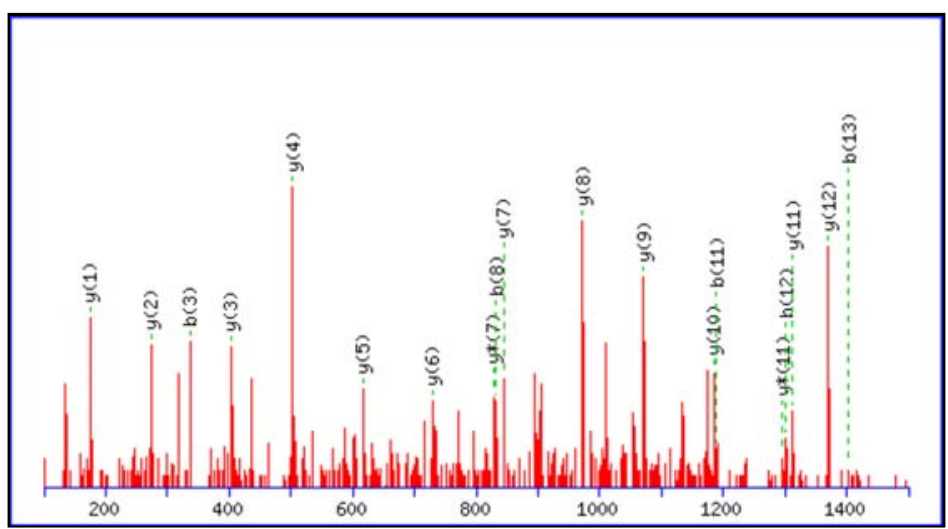

Monoisotopic mass of neutral peptide $\mathrm{Mr}$ (calc): 1802.9890

Ions Score: 78 Expect: $6.6 \mathrm{e}-005$

Matches (Bold Red): 19/162 fragment ions using 34 most intense peaks

\begin{tabular}{|c|c|c|c|c|c|c|c|c|c|c|c|c|c|c|}
\hline \# & b & $b^{++}$ & $b *$ & $b *^{++}$ & $b^{0}$ & $b^{0++}$ & Seq. & y & $\mathrm{y}^{++}$ & y* & $\mathrm{y*}^{++}$ & $y^{0}$ & $\mathrm{y}^{0++}$ & $\#$ \\
\hline 1 & 164.0706 & 82.5389 & & & & & $Y$ & & & & & & & 16 \\
\hline 2 & 265.1183 & 133.0628 & & & 47.1077 & 124.0575 & $\mathrm{~T}$ & 1640.9330 & 820.9701 & 1623.9064 & 812.4568 & 1622.9224 & 811.9648 & 15 \\
\hline 3 & 336.1554 & 168.5813 & & & 318.1448 & 159.5761 & A & 1539.8853 & 770.4463 & 1522.8587 & 761.9330 & 1521.8747 & 761.4410 & 14 \\
\hline 4 & 435.2238 & 218.1155 & & & 417.2132 & 209.1103 & V & 1468.8482 & 734.9277 & 1451.8216 & 726.4145 & 1450.8376 & 725.9224 & 13 \\
\hline 5 & 492.2453 & 246.6263 & & & 474.2347 & 237.6210 & $G$ & 1369.7798 & 685.3935 & 1352.7532 & 676.8802 & 1351.7692 & 676.3882 & 12 \\
\hline 6 & 620.3038 & 310.6556 & 603.2773 & 302.1423 & 602.2933 & 301.6503 & $Q$ & 1312.7583 & 656.8828 & 1295.7318 & 648.3695 & 1294.7477 & 647.8775 & 11 \\
\hline 7 & 733.3879 & 367.1976 & 716.3614 & 358.6843 & 715.3773 & 358.1923 & L & 1184.6997 & 592.8535 & 1167.6732 & 584.3402 & 1166.6892 & 583.8482 & 10 \\
\hline 8 & 832.4563 & 416.7318 & 815.4298 & 408.2185 & 814.4458 & 407.7265 & V & 1071.6157 & 536.3115 & 1054.5891 & 527.7982 & 1053.6051 & 527.3062 & 9 \\
\hline 9 & 960.5149 & 480.7611 & 943.4883 & 472.2478 & 942.5043 & 471.7558 & $Q$ & 972.5473 & 486.7773 & 955.5207 & 478.2640 & 954.5367 & 477.7720 & 8 \\
\hline 10 & 1075.5418 & 538.2746 & 1058.5153 & 529.7613 & 1057.5313 & 529.2693 & D & 844.4887 & 422.7480 & 827.4621 & 414.2347 & 826.4781 & 413.7427 & 7 \\
\hline 11 & 1188.6259 & 594.8166 & 1171.5993 & 586.3033 & 1170.6153 & 585.8113 & L & 729.4617 & 365.2345 & 712.4352 & 356.7212 & 711.4512 & 356.2292 & 6 \\
\hline 12 & 1301.7100 & 651.3586 & 1284.6834 & 642.8453 & 1283.6994 & 642.3533 & L & 616.3777 & 308.6925 & 599.3511 & 300.1792 & 598.3671 & 299.6872 & 5 \\
\hline 13 & 1402.7576 & 701.8825 & 1385.7311 & 693.3692 & 1384.7471 & 692.8772 & $\mathrm{~T}$ & 503.2936 & 252.1504 & 486.2671 & 243.6372 & 485.2831 & 243.1452 & 4 \\
\hline 14 & 1530.8162 & 765.9117 & 1513.7897 & 757.3985 & 1512.8057 & 756.9065 & $Q$ & 402.2459 & 201.6266 & 385.2194 & 193.1133 & & & 3 \\
\hline 15 & 1629.8846 & 815.4460 & 1612.8581 & 806.9327 & 1611.8741 & 806.4407 & V & 274.1874 & 137.5973 & 257.1608 & 129.0840 & & & 2 \\
\hline 16 & & & & & & & $\mathrm{R}$ & 175.1190 & 88.0631 & 158.0924 & 79.5498 & & & 1 \\
\hline
\end{tabular}
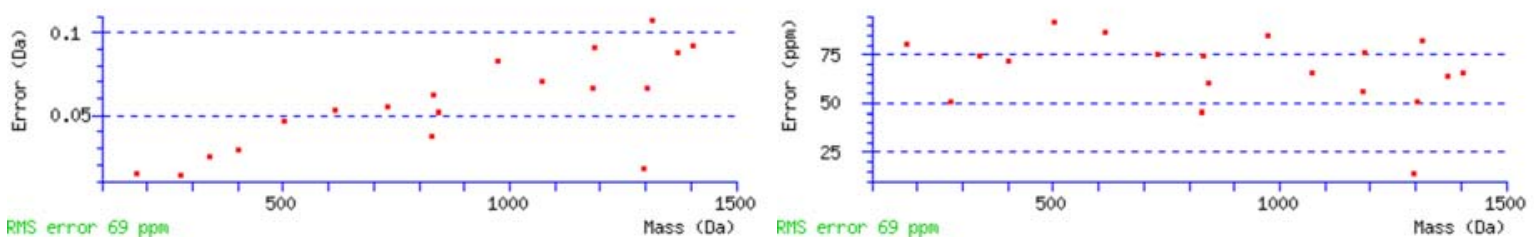


\section{Peptide View}

MS/MS Fragmentation of YVQFLSGLLSGAMK

Found in gi|94389897|ref|XP_109868.6|, PREDICTED: tensin 3 [Mus musculus]

Match to Query 476: 1512.813848 from(757.414200,2+)

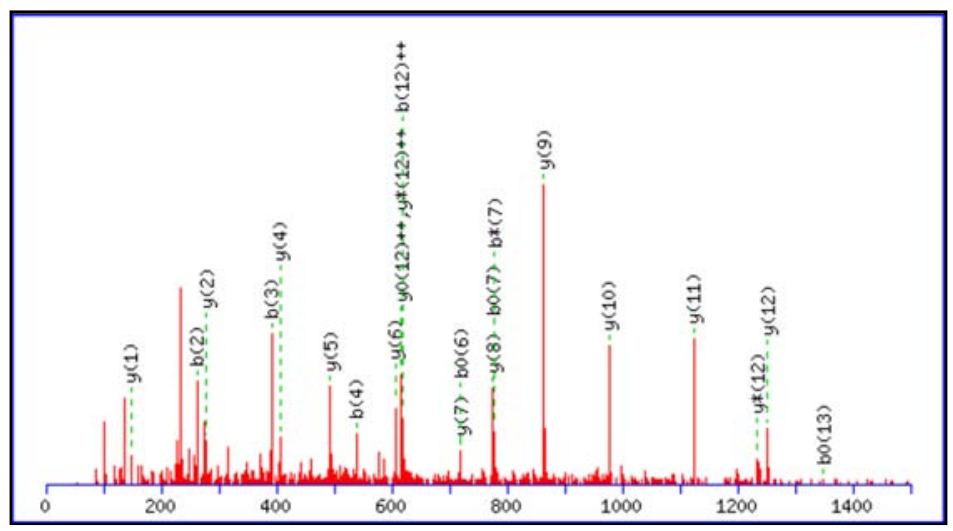

Monoisotopic mass of neutral peptide $\operatorname{Mr}$ (calc): 1512.8010

Ions Score: 68 Expect: 0.00029

Matches (Bold Red): 22/134 fragment ions using 52 most intense peaks

\begin{tabular}{|c|c|c|c|c|c|c|c|c|c|c|c|c|c|c|}
\hline \# & b & $b^{++}$ & $b *$ & $b *^{++}$ & $b^{0}$ & $b^{0++}$ & Seq. & y & $y^{++}$ & $y *$ & $\mathrm{y}^{++}$ & $y^{0}$ & $y^{0++}$ & \# \\
\hline 1 & 164.0706 & 82.5389 & & & & & $\mathrm{Y}$ & & & & & & & 14 \\
\hline 2 & 263.1390 & 132.0731 & & & & & V & 350.7450 & 675.8761 & 1333.7184 & 667.3628 & 1332.7344 & 666.8708 & . \\
\hline 3 & 391.1976 & 196.1024 & 374.1710 & 187.5892 & & & Q & 1251.6765 & 626.3419 & 1234.6500 & 617.8286 & 1233.6660 & 617.3366 & 12 \\
\hline 4 & 538.2660 & 9.6366 & 521.2395 & 261.1234 & & & $\mathrm{~F}$ & 1123.6180 & 562.3126 & 1106.5914 & 553.7993 & 1105.6074 & 553.3073 & 1 \\
\hline 5 & 651.3501 & 326.1787 & 634.3235 & 317.6654 & & & $\mathrm{~L}$ & 976.5496 & 488.7784 & 959.5230 & 480.2651 & 958.5390 & 479.7731 & 10 \\
\hline 6 & 738.3821 & 369.6947 & 721.3555 & 361.1814 & 20.3715 & 894 & $S$ & 3.4655 & 432.2364 & 846.4389 & 423.7231 & 845.4549 & 423.2311 & \\
\hline 7 & 795.4036 & 398.2054 & 778.3770 & 389.6921 & 777.3930 & 389.2001 & $G$ & 776.4335 & 388.7204 & 759.4069 & 380.2071 & 758.4229 & 379.7151 & 8 \\
\hline 8 & 908.4876 & 454.7474 & 891.4611 & 446.2342 & 890.4771 & 445.7422 & L & 719.4120 & 360.2096 & 702.3855 & 351.6964 & 701.4014 & 351.2044 & 7 \\
\hline 9 & 1021.5717 & 511.2895 & 1004.5451 & 502.7762 & 1003.5611 & 502.2842 & $\mathrm{~L}$ & 606.3279 & 303.6676 & 589.3014 & 295.1543 & 588.3174 & 294.6623 & 6 \\
\hline 10 & 1108.6037 & 554.8055 & 1091.5772 & 546.2922 & 1090.5931 & 545.8002 & $\mathrm{~s}$ & 2439 & 247.1256 & 476.2173 & 238.6123 & 475.2333 & 238.1203 & se \\
\hline 11 & 1165.6252 & 583.3162 & 1148.5986 & 574.8029 & 1147.6146 & 574.3109 & G & 406.2119 & 203.6096 & 389.1853 & 195.0963 & & & 2 \\
\hline 12 & 1236.6623 & 618.8348 & 1219.6357 & 610.3215 & 1218.6517 & 609.8295 & A & 349.1904 & 175.0988 & 332.1638 & \begin{tabular}{|l|l|}
166.5856 \\
\end{tabular} & & & 3 \\
\hline 13 & 1367.7028 & 684.3550 & 1350.6762 & 675.8417 & 1349.6922 & 675.3497 & M & 278.1533 & 139.5803 & 261.1267 & 131.0670 & & & . \\
\hline 14 & & & & & & & $\mathrm{~K}$ & 147.1128 & 74.0600 & 130.0863 & 65.5468 & & & \\
\hline
\end{tabular}
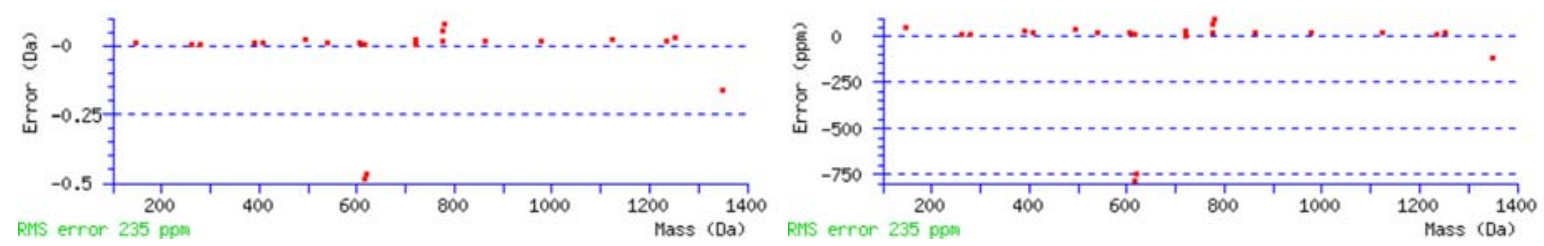